\title{
Introdução à Análise Não Standard
}

\author{
Geovani Pereira Machado
}

\author{
DISSERTAÇÃO APRESENTADA \\ AO \\ INSTITUTO DE MATEMÁTICA E ESTATÍSTICA \\ DA \\ UNIVERSIDADE DE SÃO PAULO \\ PARA \\ OBTENÇÃO DO TÍTULO DE \\ MESTRE EM MATEMÁTICA
}

Programa: Mestrado em Matemática

Orientador: Prof. Dr. Rogério Augusto dos Santos Fajardo 



\section{Introdução à Análise Não Standard}

Esta versão da dissertação contém as correções e alterações sugeridas pela Comissão Julgadora durante a defesa da versão original do trabalho, realizada em 07/12/2018. Uma cópia da versão original está disponível no Instituto de Matemática e Estatística da Universidade de São Paulo.

Comissão Julgadora:

- Prof. Dr. Rogério Augusto dos Santos Fajardo (orientador) - IME-USP

- Prof. Dr. Hércules de Araújo Feitosa - UNESP

- Prof. Dr. Leandro Fiorini Aurichi - ICMC 



\section{Agradecimentos}

Agradeço pelo generoso e incessante apoio que recebi da minha família e meus amigos durante minha trajetória acadêmica. O amor, o incentivo e o suporte concedidos por essas pessoas extraordinárias foram fundamentais para que eu pudesse superar cada obstáculo dessa longa missão. Também dedico minha sincera gratidão ao meu orientador, Prof. Rogério Augusto dos Santos Fajardo, por ter acreditado na proposta do meu trabalho e ter aceitado o desafio de me guiar nesse prazeroso e gratificante processo de aprendizado. 

"Penso que nos séculos vindouros será considerada uma grande estranheza na História da Matemática que a primeira teoria exata dos infinitesimais foi desenvolvida 300 anos após a invenção do Cálculo Diferencial." [Abraham Robinson, (45); Tradução nossa] 



\section{Resumo}

A área conhecida como Análise Não Standard consiste na aplicação dos métodos da Teoria dos Modelos e da Teoria dos Ultrafiltros para a obtenção de extensões peculiares de sistemas matemáticos infinitos. As novas estruturas construídas segundo esse procedimento satisfazem ao Princípio da Transferência, uma propriedade de suma importância e influência a qual afirma que as mesmas sentenças de primeira ordem com quantificadores limitados são verdadeiras para o sistema original e a sua extensão. Concebida em 1961 por Abraham Robinson e aprimorada por vários matemáticos nos anos subsequentes, tal área de pesquisa provou ser bastante proveitosa e esclarecedora para diversas outras partes da Matemática, como a Topologia, a Teoria das Probabilidades, a Análise Funcional e a Análise Complexa. Manifesta-se uma reavaliação da Teoria dos Domínios Ordenados seguida de um tratamento completo e gradual das fundações da Análise Não Standard assumindo a perspectiva dos Monomorfismos Não Standard, onde adota-se como metateoria a teoria dos conjuntos de Neumann-Bernays-Gödel com o Axioma da Escolha. A fim de impulsionar a assimilação da metodologia abordada, o estudo explora as propriedades do corpo não arquimediano dos números hiper-reais de maneira intuitiva e informal, utilizando-se destas para revelar demonstrações alternativas e relativamente diretas de alguns dos principais resultados do Cálculo Diferencial e Integral, como o Teorema do Valor Intermediário, o Teorema de Bolzano-Weierstrass, o Teorema do Ponto Crítico, o Teorema da Função Inversa e o Teorema Fundamental do Cálculo.

Descritores: Domínios Ordenados; Corpos Não Arquimedianos; Números Hiper-reais; Cálculo Diferencial e Integral; Ultrafiltros; Análise Não Standard. 



\section{Abstract}

The field known as Non-standard Analysis consists in the application of the methods of Model Theory and Ultrafilter Theory to the attainment of peculiar extensions of infinite mathematical systems. The new structures produced under that procedure satisfy the Transfer Principle, a property of the utmost importance and influence which states that the same first-order sentences with bounded quantifiers are true for the original system and its extension. Conceived in 1961 by Abraham Robinson and improved by a number of mathematicians in the following years, such area of research has proved to be very fruitful and illuminating to many other parts of Mathematics, such as Topology, Probability Theory, Functional Analysis and Complex Analysis. The work presents a reexamination of the Theory of Ordered Domains followed by a thorough and gradual treatment of the foundations of Non-standard Analysis under the perspective of Non-standard Monomorphisms, where Neumann-Bernays-Gödel's set theory with the Axiom of Choice is adopted as metatheory. In order to boost the assimilation of the methodology put forward, the study explores the properties of the non-archimedean field of hyperreal numbers in an intuitive and informal fashion, employing them to reveal alternative and relatively direct proofs of some of the main results of Differential and Integral Calculus, such as the Inter-

mediate Value Theorem, the Bolzano-Weierstrass Theorem, the Extreme Value Theorem, the Inverse Function Theorem and the Fundamental Theorem of Calculus.

Key words: Ordered Domains; Non-archimedean Fields; Hyperreal Numbers; Differential and Integral Calculus; Ultrafilters; Non-standard Analysis. 



\section{Sumário}

Lista de tabelas $\ldots \ldots \ldots \ldots \ldots \ldots$ xiii

Lista de Símbolos . . . . . . . . . . . . . . . xv

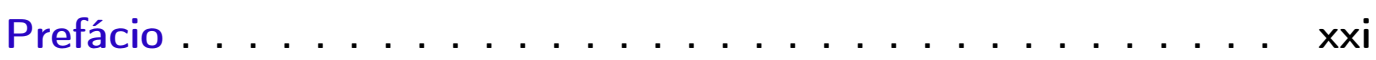

1 DOMÍNIOS E CORPOS ORDENADOS . . . . . . . . . . 1

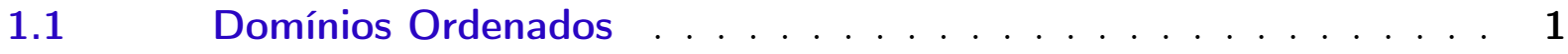

$1.2 \quad$ Corpos Ordenados . . . . . . . . . . . . . . 8

$1.3 \quad$ A Propriedade Arquimediana . . . . . . . . . . . . . 11

1.4 Relações Confrontantes em Domínios Ordenados . . . . . . . . . 15

1.5 Ideais Ordenados . . . . . . . . . . . . . . . 17

$1.6 \quad$ Mônadas e Galáxias . . . . . . . . . . . . . . . . . . 19

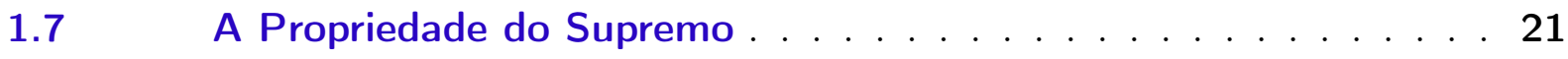

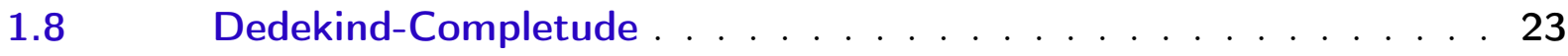

1.9 Cauchy-Completude . . . . . . . . . . . . . . . 26

1.10 Domínios Ordenados que Estendem $\mathbb{R} \ldots \ldots$. . . . . . . 32

$1.11 \quad$ Análise Diferencial e Integral em Extensões de $\mathbb{R} \ldots \ldots . . .34$

2 VISÃO INGÊNUA DOS NÚMEROS HIPER-REAIS . . . . . . . 35

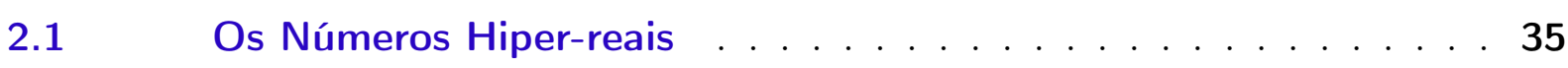

$2.2 \quad$ Objetos Relacionados a $\mathbb{R}$ e ${ }^{*} \mathbb{R} \ldots \ldots \ldots \ldots$

2.3 Condições com Quantificadores Limitados . . . . . . . . . . . . . 37

$2.4 \quad *$-Transformações e o Princípio da Transferência . . . . . . . . 38

2.5 Objetos Standard e Não Standard . . . . . . . . . . . . . . 39

2.6 Propriedades Conjuntistas de $* \ldots \ldots \ldots$. . . . . . 41

2.7 Domínios e Corpos Ordenados Relacionados a ${ }^{*} \mathbb{R} \ldots$. . . . . . . 43

2.8 Definição Alternativa para $\mathbb{R} \ldots \ldots \ldots \ldots$

2.9 Objetos Internos e Externos . . . . . . . . . . . . 47

2.10 Aplicações no Cálculo Diferencial . . . . . . . . . . . 53

2.11 Somas Hiperfinitas . . . . . . . . . . . . . . . . . . 65

2.12 Atalho Dedutivo Usual . . . . . . . . . . . . . 68

2.13 Aplicações na Riemann-Integrabilidade . . . . . . . . . . . 70

3 FILTROS E ULTRAFILTROS . . . . . . . . . . . . 75

$3.1 \quad$ A Ideia da Construção de ${ }^{*} \mathbb{R} \ldots \ldots \ldots \ldots 75$

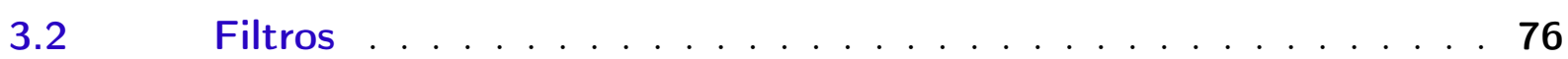


$3.3 \quad$ Produtos Reduzidos . . . . . . . . . . . . . . . . . . . . . . 82

3.4 Limites Generalizados . . . . . . . . . . . . . . . . . . 86

$3.5 \quad$ Ultrafiltros . . . . . . . . . . . . . . . . . . . . . . . . 89

$3.6 \quad$ Ultraprodutos . . . . . . . . . . . . . . . . . . . 93

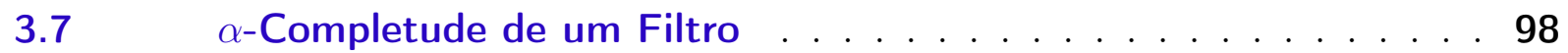

$3.8 \quad$ A Ultrapotência ${ }^{*} \mathbb{R} \ldots \ldots$. . . . . . . . . . . . . . . . 101

4 MONOMORFISMOS NÃO STANDARD . . . . . . . . . . 105

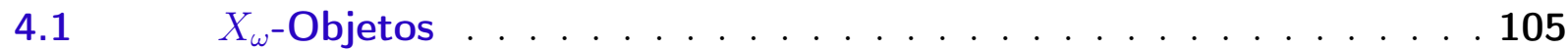

4.2 Monomorfismos Não Standard . . . . . . . . . . . . . . . . . 111

4.3 Objetos Internos e Externos . . . . . . . . . . . . . . . . . 114

4.4 Princípio Geral das $*$-Transformações . . . . . . . . . . . . . . 119

4.5 Conjuntos Hiperfinitos . . . . . . . . . . . . . . . . . . . . . 124

4.6 Operações sobre Hipersequências . . . . . . . . . . . . . . 128

4.7 Overflow e Underflow . . . . . . . . . . . . . . . . . . . 130

5 MONOMORFISMOS NÃO STANDARD EXISTEM . . . . . . . 133

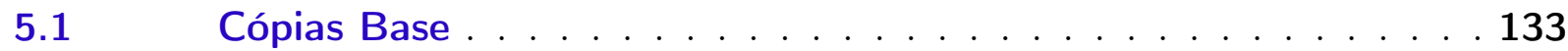

$5.2 \quad$ A Ideia da Construção de $* \ldots \ldots \ldots \ldots \ldots$

5.3 Tentativa para Definir $*_{n} \ldots \ldots \ldots$. . . . . . . . . 137

5.4 Outra Fatoração de $*_{n} \ldots \ldots \ldots 139$

5.5 Monomorfismos Não Standard Existem . . . . . . . . . . . . . . 142

$5.6 \quad * \mathbb{Q}$ e ${ }^{*} \mathbb{R}$ são Cauchy-completos . . . . . . . . . . . . . . 147

Apêndice

A CONCEITOS DA TEORIA DOS MODELOS . . . . . . . . 151

A.1 Monóides Livres . . . . . . . . . . . . . . . . . 151

A.2 Linguagens; Substituição Simultânea . . . . . . . . . . . . 152

A.3 Assinaturas . . . . . . . . . . . . . . . . . . . 152

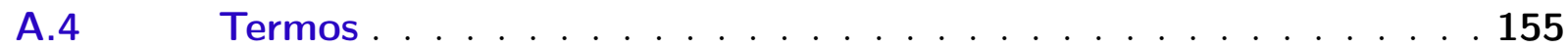

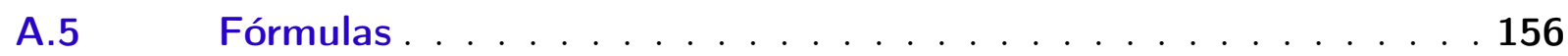

A.6 Fórmulas em $L_{\epsilon}$ com Quantificadores Limitados . . . . . . . . . 157

A.7 Variáveis Livres e Variáveis Ligadas . . . . . . . . . . . . . . . . . . 158

A.8 Substituição de Variáveis em Termos e Fórmulas . . . . . . . . . 159

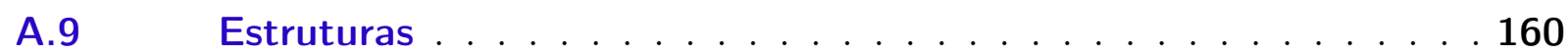

A.10 Expansões Naturais . . . . . . . . . . . . . . . . 161

A.11 Interpretação de Termos Fechados . . . . . . . . . . . . . 163

A.12 Relação de Satisfatibilidade . . . . . . . . . . . . . . . . . . . . 164

A.13 Teorias . . . . . . . . . . . . . . . . . . . . 166 
A.14 Subestruturas e Subestruturas Elementares . . . . . . . . . . . 167

A.15 Morfismos Entre Estruturas . . . . . . . . . . . . . . . . . 167

B FORMALIZAÇÕES DA TEORIA DOS CONJUNTOS . . . . . 171

B.1 As Teorias ZF, ZFC e NBG . . . . . . . . . . . . 171

B.2 Alguns Teoremas Básicos de NBG . . . . . . . . . . . . 176

B.3 Ordinais . . . . . . . . . . . . . . . . 177

B.4 Cardinais . . . . . . . . . . . . . . . . 182

B.5 Hierarquia Cumulativa dos Conjuntos . . . . . . . . . . . 183

REFERÊNCIAS ..................... . . 185

Índice . . . . . . . . . . . . . . . . . . . . . . 189 



\section{Lista de tabelas}

Tabela 1 - Operações aritméticas entre elementos infinitesimais, finitos, infinitos e apreciáveis . . . . . . . . . . . . . 12

Tabela 2 - Classificação dos domínios ordenados . . . . . . . . . . . . . . 32

Tabela 3 - Classificação dos corpos ordenados . . . . . . . . . . . . . . 32

Tabela 4 - Correspondências entre os postulados do Teorema 2.3 e os axiomas, convenções e teoremas do Capítulo 4. . . . . . . . . . . . 113 



\section{Lista de símbolos}

$C\left(x_{1} \ldots x_{n}\right) \quad$ Condição $C\left(x_{1} \ldots x_{n}\right)$ nas variáveis $x_{1} \ldots x_{n}$, a qual é uma $L_{\epsilon}$-fórmula de primeira ordem (Exemplo A.7 e Definição A.14) na metalinguagem usada nesta dissertação. A metateoria que adotaremos nessa metalinguagem será a Teoria dos Conjuntos descrita pelos axiomas de NBG (Seção B.1).

$\neg C\left(x_{1} \ldots x_{n}\right) \quad$ Negação de uma condição $C\left(x_{1} \ldots x_{n}\right)$ nas variáveis $x_{1} \ldots x_{n}$.

$C_{1} \vee C_{2} ; C_{1}$ ou $C_{2} \quad$ Disjunção inclusiva das condições $C_{1}$ e $C_{2}$, a qual será considerada verdadeira se, e somente se, pelo menos uma das condições $C_{1}$ e $C_{2}$ for verdadeira.

$C_{1} \wedge C_{2} ; C_{1}$ e $C_{2} \quad$ Conjunção das condições $C_{1}$ e $C_{2}$, a qual será considerada verdadeira se, e somente se, ambas as condições $C_{1}$ e $C_{2}$ forem verdadeiras.

$C_{1} \Rightarrow C_{2} \quad$ A condição $C_{1}$ implica na condição $C_{2}$, ou seja, $C_{2}$ será verdadeira sempre que $C_{1}$ for verdadeira.

$C_{1} \Leftrightarrow C_{2} \quad$ A condição $C_{1}$ é equivalente à condição $C_{2}$, ou seja, $C_{1}$ será verdadeira exatamente quando $C_{2}$ for verdadeira.

$C_{1}: \Leftrightarrow C_{2} \quad$ A condição $C_{1}$ é definida como sendo equivalente à condição $C_{2}$.

$A:=B \quad A$ é definido como sendo igual ao objeto $B$.

$\in \quad$ Relação de pertinência da Teoria dos Conjuntos.

$\subset \quad$ Relação de inclusão não estrita entre classes.

$\subsetneq \quad$ Relação de inclusão estrita ou própria entre classes.

$\notin, \not \subset, \not \subset$, etc. $\quad$ Negações das relações de pertinência, de inclusão não estrita, e de inclusão estrita, respectivamente.

$\{F(x)\}_{P(x)},\{F(x) \vdots P(x)\} \quad$ Classe dos objetos da forma $F(x)$ para objetos $x$ que satisfazem à propriedade $P(x)$, a qual pode ser um conjunto ou uma classe própria dependendo da propriedade $P(x)$. Os sistemas formais usuais que formalizam a Teoria dos Conjuntos (ZF, ZFC, NBG, etc.) limitam as opções de propriedades $P(x)$ que podem ser usadas para definir uma classe. Conjuntos contáveis às vezes são descritos elemento a elemento, usando a notação $\left\{x_{1}, x_{2}, \ldots, x_{n}, \ldots\right\}$ ou $\left\{x_{1} x_{2} \ldots x_{n} \ldots\right\}$. 
$\left\{X_{i}\right\}_{P(i)},\left\{X_{i} \vdots P(i)\right\} \quad$ Embora sejam essencialmente iguais às notações que definem classes, essas representações às vezes denotam a família (i.e., a função) cuja classe de índices é

$$
I:=\{i: P(i)\}
$$

e cuja regra de associação é $i \mapsto X_{i}$. Quando estiverem inseridas em expressões matemáticas simbólicas na presença de outros símbolos matemáticos (como $\in, \subset, \cap$, etc.), elas remeterão à classe subentendida. Quando estiverem isoladas no texto, será explicitamente mencionado no contexto se tais notações representam uma classe ou uma família.

$\bigcup_{i \in I} A_{i}=\bigcup\left\{A_{i}\right\}_{i \in I} \quad$ União da família de conjuntos $\left\{A_{i}\right\}_{i \in I}$, definida por

$$
\bigcup_{i \in I} A_{i}:=\left\{x \vdots(\exists i \in I) x \in A_{i}\right\} .
$$

$\bigcap_{i \in I} A_{i}=\bigcap\left\{A_{i}\right\}_{i \in I} \quad$ Interseção da família de conjuntos $\left\{A_{i}\right\}_{i \in I}$, definida por

$$
\bigcap_{i \in I} A_{i}:=\left\{x:(\forall i \in I) x \in A_{i}\right\} .
$$

Em NBG, temos $\bigcap_{i \in \emptyset} A_{i}=\bigcap \emptyset=\mathrm{V}$.

$A-B \quad$ Diferença (assimétrica) entre as classes $A$ e $B$, definida por

$$
A-B:=\{x \in A \vdots x \notin B\} \text {. }
$$

$\mathcal{P}(A) \quad$ Conjunto Potência ou conjunto das partes de um conjunto $A$, definido por

$$
\mathcal{P}(A):=\{x: x \subset A\}
$$

Denotaremos por $\mathcal{P}^{n}(A)$ a $n$-ésima iteração da operação $\mathcal{P}$ sobre o conjunto $A$, ou seja:

$$
\mathcal{P}^{n}(A):=\overbrace{\mathcal{P}(\cdots(\mathcal{P}(\mathcal{P}}^{n \text { vezes }}(A))) \cdots),
$$

onde o caso $n=0$ é definido por $\mathcal{P}^{0}(A):=A$.

$\emptyset \quad$ Conjunto vazio, definido por

$$
\emptyset:=\{x: x \neq x\} .
$$


Universo de von Neumann, definida como sendo a classe dos conjuntos, a qual é dada por

$$
\mathrm{V}=\{x: x=x\}
$$

$\mathbb{N}:=\{1,2,3,4, \ldots\} \quad$ Conjunto dos números naturais.

$\mathbb{N}_{0}:=\{0,1,2,3,4, \ldots\} \quad$ Conjunto cujos elementos são os números naturais e o número zero.

$\mathbb{Z}:=\{\ldots,-2,-1,0,1,2, \ldots\} \quad$ Conjunto dos números inteiros.

$\mathbb{Q} \quad$ Conjunto dos números racionais.

Irr Conjunto dos números irracionais.

$\mathbb{R} \quad$ Conjunto dos números reais.

$(a, b) \quad$ Par ordenado de Kuratowski, definido por:

$$
(a, b):=\{\{a\},\{a, b\}\}
$$

Para objetos matemáticos $a_{1} \ldots a_{n}$, a $n$-tupla ordenada $\left(a_{1} \ldots a_{n}\right)$ é definida indutivamente como segue:

- $\left(a_{1}\right):=a_{1}$;

- $\left(a_{1}, \ldots, a_{n}, a_{n+1}\right):=\left(\left(a_{1}, \ldots, a_{n}\right), a_{n+1}\right)$ para $n \geqslant 1$.

$a_{k} a_{k+1} \ldots a_{k+n} \ldots \quad$ Sequência de objetos com índice finito ou enumerável, definida por

$$
a_{k} a_{k+1} \ldots a_{k+n} \cdots:=\left\{\left(k, a_{k}\right),\left(k+1, a_{k+1}\right), \cdots,\left(k+n, a_{k+n}\right), \cdots\right\} .
$$

Incluiremos vírgulas entre os objetos de uma sequência com uma quantidade indeterminada de termos (e.g. com $n$ elementos) apenas quando houver possibilidade de confusão na leitura dos termos. Analogamente, quando a quantidade de termos for indeterminada e não houver risco de confusão, omitiremos as vírgulas nas notações de conjuntos contáveis $\left(\left\{a_{1} a_{2} a_{3} \ldots\right\}\right)$ e de $n$-tuplas ordenadas $\left(\left(a_{1} a_{2} \ldots a_{n}\right)\right)$. Nos casos $k=0$ ou $k=1$, denotaremos as sequências

$$
a_{0} a_{1} a_{2} \ldots \text { e } a_{1} a_{2} a_{3} \ldots
$$

simplesmente por $\left\{a_{n}\right\}$, onde o contexto deve deixar claro se os índices começam em $k=0$ ou em $k=1$. 
$a_{k} a_{k+1} \ldots a_{k+n} \in A \quad$ Conjunção das $n+1$ condições

$$
a_{k} \in A, a_{k+1} \in A, \ldots, a_{k+n} \in A
$$

A afirmação que a sequência $a_{k} a_{k+1} \ldots a_{k+n}$, vista como uma função, pertence ao conjunto $A$ sempre será declarada verbalmente sem a utilização do símbolo da relação de pertinência.

$A_{1} \cup A_{2} \cup \cdots \cup A_{n} \cup \cdots \quad$ União enumerável da sequência $A_{1} A_{2} \ldots A_{n} \ldots$ de conjuntos, definida analogamente à união de uma família.

$A_{1} \cap A_{2} \cap \cdots \cap A_{n} \cap \cdots \quad$ Interseção enumerável da sequência $A_{1} A_{2} \ldots A_{n} \ldots$ de conjuntos, definida analogamente à interseção de uma família.

$A_{1} \times A_{2} \times \cdots \times A_{n} \quad$ Produto cartesiano finito de uma sequência finita de conjuntos $A_{1} A_{2} \ldots A_{n}$, definido por

$$
A_{1} \times A_{2} \times \cdots \times A_{n}:=\left\{\left(a_{1} a_{2} \ldots a_{n}\right) \vdots a_{1} \in A_{1}, a_{2} \in A_{2}, \ldots, a_{n} \in A_{n}\right\} .
$$

Note que

$$
A_{1} \times A_{2} \times \cdots \times A_{n}=\left(\left(\left(A_{1} \times A_{2}\right) \times \cdots\right) \times A_{n-1}\right) \times A_{n} .
$$

$\mathrm{E}_{X} \quad$ Relação de pertinência em uma classe $X$, definida por

$$
\mathrm{E}_{X}:=\{(x, y) \in X \times X: x \in y\}
$$

$\operatorname{dom}(R) \quad$ Domínio de uma relação $R$, definido por

$$
\operatorname{dom}(R):=\{x \vdots(\exists y)(x, y) \in R\} .
$$

$\operatorname{Im}(R) \quad$ Imagem de uma relação $R$, definida por

$$
\operatorname{Im}(R)=\{y \vdots(\exists x)(x, y) \in R\} .
$$

$R\langle A\rangle \quad$ Imagem de $A$ sobre uma relação $R$, definida por

$$
R\langle A\rangle:=\{y \vdots(\exists x \in A)(x, y) \in R\}
$$

$R \circ S \quad$ Composição de uma relação $R$ com uma relação $S$, definida por

$$
R \circ S:=\{(x, z) \vdots(\exists y)((x, y) \in S \wedge(y, z) \in R)\} .
$$


$A / \sim \quad$ Quociente de um conjunto $A$ por uma relação de equivalência $\sim$ em $A$, definida por

$$
A / \sim:=\{\sim\langle\{x\}\rangle: x \in A\}
$$

$f: A \rightarrow B \quad f$ é uma função com domínio $A$ cujos valores pertencem ao contradomínio $B$. Isso significa que $f \subset A \times B$ e $f$ satisfaz à condição

$$
(\forall x \in A)(\exists ! y \in B)(x, y) \in f .
$$

${ }^{A} B \quad$ Conjunto das funções $f$ do tipo $A \rightarrow B$, onde $A$ e $B$ são conjuntos.

$\operatorname{id}_{A} \quad$ Função identidade $A \rightarrow A$, definida por

$$
\operatorname{id}_{A}(x):=x(\forall x \in A) .
$$

$\prod_{i \in I} A_{i} \quad$ Produto cartesiano de uma família de conjuntos $\left\{A_{i}\right\}_{i \in I}$, definido por

$$
\prod_{i \in I} A_{i}:=\left\{f: I \rightarrow \bigcup_{i \in I} A_{i}:(\forall i \in I) f(i) \in A_{i}\right\} .
$$

Se $A_{i}=A(\forall i \in I)$, então definimos ${ }^{I} A:=\prod_{i \in I} A_{i}$.

$|A| \quad$ Cardinal do conjunto $A$, definido como sendo o único ordinal inicial equipotente a $A$.

$\mathcal{P}_{\alpha}(A) \quad$ Conjunto das partes $x$ de um conjunto $A$ tais que $|x|<\alpha$, onde $\alpha$ é um cardinal.

$(a, b)_{(X, R)} \quad$ Notação para intervalos abertos relativos a uma relação binária $R$ em uma classe $X$, definida por

$$
(a, b)_{(X, R)}:= \begin{cases}\{x \in X \vdots(a, x) \in R \text { e }(x, b) \in R\} & \text { se }(a, b) \in R \\ \emptyset & \text { se }(a, b) \notin R,\end{cases}
$$

onde $a, b \in X$. Quando a relação $R$ em $X$ já estiver implícita no contexto, denotaremos o intervalo $(a, b)_{(X, R)}$ simplesmente por $(a, b)_{X}$. As notações $[a, b]_{X},(a, b]_{X}$, e $[a, b)_{X}$ são definidas de modo análogo concordando com a simbologia usual para intervalos.

$\operatorname{Int}(A, R) B \quad$ Classe das funções que têm o conjunto $B$ como contradomínio e cujos domínios são intervalos fechados relativos a uma relação binária $R$ em um conjunto $A$, definida por

$$
\operatorname{Int}(A, R) B:=\bigcup_{a, b \in A}[a, b] B
$$


onde

$$
[a, b]:= \begin{cases}\{a, b\} \cup\{x \in A \vdots(a, x) \in R \text { e }(x, b) \in R\} & \text { se }(a, b) \in R \\ \emptyset & \text { se }(a, b) \notin R .\end{cases}
$$

$\operatorname{SeqF}(X) \quad$ Classe das sequências finitas de elementos de uma classe $X$ com índices em segmentos iniciais de $\mathbb{N}$, definida por

$$
\operatorname{SeqF}(X):=\bigcup_{n=0}^{\infty}[1, n] X
$$

onde $[1, n]=[1, n]_{\mathbb{N}_{0}}$. Note que ${ }^{[1,0]} X={ }^{\emptyset} X=\{\emptyset\}$ e $\emptyset \in \operatorname{SeqF}(X)$. 


\section{Prefácio}

A noção das quantidades infinitesimais, entendidas como números que em módulo são menores que qualquer número real positivo, surgiu e desapareceu diversas vezes no decorrer da História da Matemática, de forma explícita e implícita (2, 8, 10, 25, 29). O mesmo pode ser dito da ideia das quantidades infinitas, entendidas como números que em módulo são maiores que qualquer número real positivo. Por serem aparentemente autocontraditórios, muitos rejeitaram tais conceitos com veemência e dedicação, ${ }^{1}$ enquanto outros, principalmente físicos e engenheiros, os interpretavam como "ficções úteis" facilitadoras do processo criativo de descoberta científica e matemática.

O primeiro matemático a propor a existência de uma extensão consistente dos números reais que contém quantidades infinitesimais e infinitas foi Gottfried Wilhelm von Leibniz (1646-1716 A.D.). Este foi um polímata e filósofo alemão que ocupa uma posição proeminente na História da Matemática e da Filosofia (18, 30, 34, 44, 51), e seu trabalho influenciou decisivamente gerações de pensadores em diversas áreas do conhecimento. Sua obra mais conhecida, a criação e o desenvolvimento das fundações do Cálculo Diferencial e Integral, impactou profundamente a concepção de Matemática do seu tempo.

O sistema concebido por Leibniz ${ }^{2}$ consiste em um conjunto de regras que descrevem as propriedades dos novos números ideais, os quais podem ser entendidos como variáveis ou constantes dependendo do contexto. Entre tais regras, destaca-se o princípio heurístico fundamental Lex Continuitatis $(2,3,4,30,51)$, o qual postula que qualquer fato que for verdadeiro para os números finitos, estes entendidos como quantidades mensuráveis com instrumentos reais de medida, também será válido para os números ideais. Dessa maneira, resultados que envolvem funções transcendentais, como por exemplo a identidade trigonométrica

$$
\operatorname{sen}^{2}(x)+\cos ^{2}(x)=1,
$$

também serão verdadeiros quando as suas variáveis assumirem valores na classe dos números ideais. Isso implica que todas as funções matemáticas definidas sobre valores finitos têm uma extensão para o novo sistema numérico.

Utilizando-se desse sistema, Leibniz obteve resultados importantes da disciplina conhecida atualmente como Cálculo Diferencial e Integral (ou simplesmente Cálculo), como as regras de derivação para somas, produtos, quocientes, potências e raízes, as propriedades básicas da integração e o Teorema Fundamental do Cálculo. Alguns dos seus termos e notações, considerados simplificadores e esclarecedores, foram estandardizados

1 Mais notavelmente George Berkeley (1685-1753 A.D.) (4, 5), Georg Cantor (1845-1918 A.D.) (4, 17), e Bertrand Russell (1872-1970 A.D.) (4, 47).

2 Para uma exposição detalhada, ver (9). 
pela comunidade matemática até os dias atuais, como o termo "função" e as notações $\mathrm{d} y / \mathrm{d} x$ e $\int y \mathrm{~d} x$, e talvez sejam seu legado matemático mais duradouro (11).

Vale mencionar que iniciou-se no ano 1699 uma disputa acirrada entre o físico e matemático inglês Isaac Newton (1642-1727 A.D.) (e seus seguidores) e Leibniz (e seus seguidores) relativa ao crédito da invenção inédita do Cálculo (26). Muitas acusações foram feitas de ambas as partes, e, mesmo após a morte de Leibniz, o debate persistiu por anos. O consenso atual entre os historiadores é que os dois pensadores, Newton e Leibniz, inventaram o Cálculo independentemente um do outro, de modo que Newton foi o primeiro a obter resultados na área, embora Leibniz tenha sido o primeiro a publicar algo sobre o assunto. Newton, no entanto, evitava o uso dos números infinitesimais sempre que possível. Isso fica claro na seguinte passagem do Commercium Epistolicum Collinii $\&$ aliorum, De Analysi promota, uma coleção de correspondências relevantes à disputa citada entre Newton e Leibniz, onde Newton (referindo-se a si mesmo na terceira pessoa) afirma (15):

\begin{abstract}
Nós não temos ideias de quantidades infinitamente pequenas. Por isso, o Sr. Newton introduz fluxões em seu método que procederiam ao máximo possível com quantidades finitas. Esse método é mais natural e geométrico porque se fundamenta nas razões primeiras de quantidades nascentes [primae quantitatum nascentium rationes] que têm uma existência na geometria [...] [Tradução nossa]
\end{abstract}

A abordagem Newtoniana ao Cálculo teve uma concepção dinâmica sobre os processos matemáticos (10), de modo que os infinitesimais eram entendidos como movimentos contínuos evanescentes, e eventualmente se desenvolveria na teoria moderna dos limites.

A proposta de Leibniz inspirou uma série de matemáticos de primeira classe dos séculos XVII e XVIII a utilizarem os números infinitesimais e infinitos rotineiramente em seus argumentos, como Jacob Bernoulli (1655-1705 A.D.) (2, 4, 7, 8, 30), Leonhard Euler (1707-1783 A.D.) (2, 3, 4, 6, 51), Joseph-Louis Lagrange (1736-1813 A.D.) (3, 7, 32), e Augustin-Louis Cauchy (1789-1857 A.D.) (2, 3, 4, 6, 7, 8, 12, 30). Tais matemáticos divergiam consideravelmente em relação às suas concepções de prova (como exemplo, Cauchy era fervorosamente contra o Princípio da Generalidade da Álgebra, o qual era amplamente aplicado por Euler e Lagrange (6, 7, 8, 30)), mas era consenso entre eles que os números infinitesimais e infinitos tinham lugar garantido na Matemática. Euler, considerado o seguidor mais eloquente dos métodos de Leibniz (23), declarou (21):

\footnotetext{
Mesmo se alguém negasse que números infinitos realmente existem neste mundo, ainda assim em especulações matemáticas surgiriam questões cujas respostas não podem ser dadas a menos que admitíssemos um número infinito. [Tradução nossa]
}

Como Leibniz e seus seguidores não forneceram uma base matemática bem fundada e convincente para o novo sistema numérico proposto, este foi tratado com muita suspeita e desconfiança por muitos matemáticos e filósofos dos séculos XVIII e XIX, culminando no gradual declínio da teoria dos ideais de Leibniz e na sua substituição pela teoria dos limites de Bernard Bolzano (1781-1848 A.D.) e Karl Weierstrass (1815-1897 
A.D.) predominante até a atualidade, também conhecida como Método $\epsilon-\delta$, cuja formulação é puramente aritmética $(2,4,7,8,30)$. Tal método permitiu que os matemáticos removessem os números infinitesimais e infinitos dos cursos de Análise, e, em meados do século XIX, tais conceitos já haviam sido expurgados da comunidade matemática, assim como a dependência dos conceitos geométricos intuitivos e dos diagramas. Nesse período, os ideais de Leibniz persistiram apenas como ajudantes intuitivos para físicos, engenheiros e matemáticos que lidavam com integrais múltiplas (36), e assim permanecem sendo tratados pela maioria da comunidade matemática contemporânea.

Apenas em meados do século XX que a ambição de Leibniz seria ressuscitada, e, desta vez, não haveriam dúvidas em relação à sua importância e consistência. No dia 24 de Janeiro de 1961, em um discurso plenário para as bodas de prata da Association for Symbolic Logic, o matemático Abraham Robinson (1918-1974 A.D.) anunciou que havia encontrado uma formalização para um sistema númerico que correspondia em vários aspectos ao concebido por Leibniz, e, em 1966, publicou seus resultados em seu aclamado livro Non-standard Analysis (45), fundando a área que ficaria conhecida como Análise Não Standard. Nessa obra, Robinson afirma:

É mostrado neste livro que as ideias de Leibniz podem ser plenamente vindicadas e que elas orientam a uma abordagem inovadora e frutífera da Análise clássica e de muitos outros ramos da Matemática. A chave para o nosso método é fornecida pela análise detalhada da relação entre as linguagens matemáticas e as estruturas matemáticas que jaz no cerne da Teoria dos Modelos contemporânea. [Tradução nossa]

Utilizando-se de métodos da Teoria dos Modelos, área dedicada ao estudo das estruturas matemáticas sob a perspectiva da Lógica Matemática, e utilizando-se do Axioma da Escolha, Robinson provou ser possível obter uma fundação matematicamente correta não apenas para uma parte significativa do sistema heurístico idealizado por Leibniz, a qual estende os números reais e ficaria conhecida como Sistema dos Hiper-reais, mas também para sistemas generalizados que estendem qualquer estrutura matemática infinita.

Diversas extensões do corpo ordenado dos números reais haviam sido descritas com sucesso antes do trabalho de Robinson (27) e várias outras foram detalhadas posteriormente $(20,22)$. Muitas dessas estruturas propostas carecem de propriedades que são consideradas cruciais para sistemas numéricos, dificultando o desenvolvimento de uma Análise Diferencial e Integral sobre tais sistemas. Duas abordagens bastante frutíferas nesse âmbito que merecem destaque são o corpo ordenado dos números surreais $(24,31,16)$ e o corpo de Levi-Civita $(48,49)$, as quais propiciaram o desenvolvimento de teorias que gozam de vários teoremas análogos aos resultados no cerne da Análise Real.

Uma das principais dificuldades ao lidar com extensões de $\mathbb{R}$ surge quando as funções transcendentais são consideradas, como, por exemplo, estender o domínio das funções $e^{x}$ e $\sin (x)$ para o novo corpo não arquimediano em questão. Mesmo que tais extensões sejam sistematicamente definidas em uma construção particular, elas não possuem, em 
geral, as principais propriedades das suas contrapartes usuais. Uma vitória significativa de Robinson foi ter revelado que toda função (transcendental ou não) sobre os reais pode ser estendida para os hiper-reais, e tais extensões naturalmente "herdam" das suas versões reais originais todas as propriedades que podem ser descritas em uma linguagem particular de primeira ordem. Assim, de certa maneira, o princípio Lex Continuitatis de Leibniz é respeitado nessas extensões.

Nas décadas subsequentes à descoberta de Robinson até a atualidade, diversos matemáticos apresentaram maneiras mais práticas e descomplicadas de lidar com o tema, ${ }^{3}$ expressando-o por intermédio de resultados mais conhecidos e tornando-o mais acessível ao público não especializado na área da Lógica e Fundamentos da Matemática. Além disso, houve um significante desenvolvimento de novos métodos que buscavam tornar a Análise Não Standard melhor aplicável às diversas partes da Matemática, tais como a Topologia, a Teoria das Probabilidades, a Análise Funcional, a Teoria das Medidas e a Análise Complexa. A despeito desse extensivo aperfeiçoamento e progresso, a pesquisa na área é relativamente nova e pouco divulgada, e muitos problemas ainda estão em aberto, abrindo amplo espaço para investigações promissoras com alto potencial para novas descobertas.

3 O próprio Robinson contribuiu significativamente nesse processo. Outros notáveis são: Elias Zakon (1908-1991 A.D.), Wilhelmus Luxemburg (1929-2018 A.D.), Edward Nelson (1932-2014 A.D.) e Keith Stroyan (vivo, nascido em 1944). 


\section{1 \\ Domínios e Corpos Ordenados}

Neste capítulo, revisaremos algumas especificidades dos domínios e corpos ordenados que serão relevantes para nossa discussão nos capítulos subsequêntes, estabelecendo uma base de terminologias e notações. Inspiradas em propriedades aritméticas, as axiomatizações dessas estruturas retratam os vínculos fundamentais entre a adição, a multiplicação e a ordem dos números. Porém, essas propriedades básicas não são suficientes para delimitar todos os atributos numéricos, e, como veremos, existe uma variedade de tipos de domínios e corpos ordenados, os quais podem ser classificados de acordo com as conjunturas que eles observam. Destacaremos o corpo ordenado dos números reais devido à sua importância no âmbito da Análise matemática.

Assumiremos a Teoria dos Conjuntos de Neumann-Bernays-Gödel (Definição B.4) como metateoria nesta dissertação. Como essa teoria é uma extensão conservativa (Definição A.39) de ZFC (Definição B.2), todos os resultados que obteremos podem ser reescritos como teoremas de ZFC.

\subsection{Domínios Ordenados}

Assumiremos que o leitor já está familiarizado com a axiomatização e as propriedades básicas dos domínios. Investigaremos os efeitos que a introdução de uma ordem e mais dois axiomas a tal estrutura trariam para a discussão.

Definição 1.1. Dizemos que um domínio $D$ é um domínio ordenado quando munido de uma ordem ${ }^{1}<$ em $D$ se para quaisquer $x, y, z \in D$ temos:

(Inv) Se $x<y$, então $x+z<y+z$;

(Invariância Translacional)

(Pos) Se $0<x$ e $0<y$, então $0<x y$.

(Positividade do Produto)

1 Estamos nos referindo à noção de ordem total estrita, ou seja, a relação $<\subset E \times E$ referida obedecerá às seguintes propriedades $(\forall x, y, z \in E)$ :

(Trans) Se $x<y$ e $y<z$, então $x<z$;

(Transitividade)

(Irr) $x \nless x$;

(Irreflexividade)

(Lin) Se $x \neq y$, então $x<y$ ou $y<x$.

(Linearidade)

A ordem não estrita correspondente a uma ordem será denotada pelo mesmo símbolo da ordem estrita exceto que haverá um traço a mais abaixo do símbolo em questão (e.g. $\leqslant$ ). Um conjunto munido de uma ordem é dito ser um conjunto ordenado. Um subconjunto $F$ de $E$ é dito ser denso em $E$ se para quaisquer $x, y \in E \operatorname{com} x<y$ existe um $z \in F$ tal que $x<z<y$. Dizemos que o conjunto ordenado $(E,<)$ é denso se $E$ é denso em $E$. 
Dizemos que $x \in D$ é positivo (resp. não negativo) se $0<x$ (resp. $0 \leqslant x$ ), e dizemos que $x$ é negativo (resp. não positivo) se $x<0$ (resp. $x \leqslant 0$ ). Se $x \in D$ e $n \in \mathbb{N}$, então denotaremos a soma

$$
\overbrace{x+x+\cdots+x}^{n \text { vezes }} \in D
$$

por $n x$. Em particular, denotaremos o elemento $n 1$ simplesmente por $n$, e o rotularemos como um número natural em $D$. Similarmente, se $m$ e $n$ forem dois números inteiros com $n$ inversível em $D$, então diremos que o elemento $m / n=m \cdot n^{-1} \in D$ é um número racional em $D$.

A implicação em (Inv) pode ser substituída por uma equivalência sem alterar o significado da condição, pois basta usar a própria condição (Inv) somando $-z$ em ambos os lados da condição $x+z<y+z$ para obter $x<y$.

Prova-se diretamente que a condição (Pos) é equivalente à condição

(InvG) Se $x<y$ e $0<z$, então $x z<y z . \quad$ (Invariância Geométrica)

Se $0<z$ e $x z<y z$, então teremos $x<y$, pois caso contrário teríamos $y \leqslant x$ e $y z \leqslant x z$ por (InvG), o que seria absurdo.

Sob a perspectiva da Teoria dos Modelos (Apêndice A), os domínios ordenados $D$ são as $L_{A O}$-estruturas (Exemplo A.10 e Definição A.23) que são modelos da $L_{A O}$-teoria gerada pelos seguintes axiomas:

1. $(\forall x, y, z) x+(y+z)=(x+y)+z$;

2. $(\forall x) x+0=x$;

3. $(\forall x)(\exists y) x+y=0$;

4. $(\forall x, y) x+y=y+x$;

5. $(\forall x, y, z) x \cdot(y \cdot z)=(x \cdot y) \cdot z$;

6. $(\forall x) x \cdot 1=x$;

7. $(\forall x, y) x \cdot y=y \cdot x$;

8. $(\forall x, y, z)(x+y) \cdot z=x \cdot z+y \cdot z$;
9. $(\forall x, y)(x \cdot y=0 \longrightarrow(x=0 \vee y=0))$;

10. $0 \neq 1$;

11. $(\forall x, y, z)((x<y \wedge y<z) \longrightarrow x<z)$;

12. $(\forall x)(\neg x<x)$;

13. $(\forall x, y)((\neg x=y) \longrightarrow(x<y \vee y<x))$;

14. $(\forall x, y, z)(x<y \longrightarrow x+z<y+z)$;

15. $(\forall x, y)((0<x \wedge 0<y) \longrightarrow 0<x \cdot y)$.

Nesta dissertação, denotaremos tal $L_{A O}$-teoria por $T_{D O}$. Os axiomas 1-4 certificam que $(D,+, 0)$ é um grupo comutativo; os axiomas 5 -7 certificam que $(D, \cdot, 1)$ é um monóide comutativo; os axiomas 1-8 certificam que $(D,+, \cdot, 0,1)$ é um anel comutativo; os axiomas 1-10 certificam que $(D,+, \cdot, 0,1)$ é um domínio; e os axiomas 11-13 garantem que $(D,<)$ é um conjunto ordenado. Neste capítulo, assumiremos que $(D,+, \cdot, 0,1,<)$ é um domínio 
ordenado, o qual será denotado apenas por $D$ por simplicidade. O domínio subentendido em $D$ também será denotado por $D$ por abuso de linguagem.

As noções de morfismo, imersão, imersão elementar e isomorfismo entre domínios ordenados são definidas como casos particulares das noções equivalentes referidas no estudo da Teoria dos Modelos (Seção A.15). Em particular, se $f: D \rightarrow E$ for um morfismo entre domínios ordenados, então a imagem de $1 \in D$ sobre $f$ será o elemento neutro multiplicativo de $E$, e, como $f$ também é um morfismo entre os respectivos grupos aditivos subentendidos em $D$ e $E$, teremos que $f(n)=n(\forall n \in \mathbb{N})$. A noção de subdomínio ordenado corresponde à noção de subestrutura (Definição A.41). O kernel de um morfismo entre domínios ordenados é a imagem inversa do conjunto $\{0\}$ sobre tal morfismo.

Observação 1.2. Na Definição 1.1, definimos os domínios ordenados com ordens estritas $(<)$, mas poderiamos tê-los definido com ordens não estritas $(\leqslant)$. Embora as duas caracterizações sejam essencialmente iguais, discrepâncias surgem ao considerarmos morfismos $f$ entre tais estruturas. Isso ocorre pois as implicações

$$
x<y \Rightarrow f(x)<f(y)
$$

e

$$
x \leqslant y \Rightarrow f(x) \leqslant f(y)
$$

não são equivalentes. Com finalidade de evitar confusões entre tais conceitos, chamaremos a estrutura de domínio ordenado com a ordem não estrita de domínio não estritamente ordenado.

Exemplo 1.3. Se $f: D \rightarrow E$ for um morfismo entre domínios não estritamente ordenados, se $A$ for um subdomínio ordenado de $D$, e se $B$ um for subdomínio ordenado de $E$, então a imagem $f\langle A\rangle$ será um subdomínio ordenado de $E$, e a imagem inversa $f^{-1}\langle B\rangle$ será um subdomínio ordenado de $D$.

Exemplo 1.4. Os domínios dos números inteiros, dos números racionais e dos números reais, os quais são respectivamente denotados por $\mathbb{Z}, \mathbb{Q}$ e $\mathbb{R}$, constituem domínios ordenados quando munidos das ordens usuais. Estes exemplos são insuspeitos dado que os axiomas (Inv) e (Pos) originam-se das propriedades desses domínios numéricos.

Exemplo 1.5. Seja $g$ um número irracional, e seja $\mathbb{Z}(g)$ o subconjunto de $\mathbb{R}$ definido por

$$
\mathbb{Z}(g):=\mathbb{Z}+g \mathbb{Z}=\{u+v g \vdots u, v \in \mathbb{Z}\}
$$

Note que 0 e 1 pertencem a $\mathbb{Z}(g)$.

Provaremos que $\mathbb{Z}(g)$ é denso em $\mathbb{R}$. Sejam $a, b \in \mathbb{R}$ com $a<b$, seja $n$ um número natural com $1 / n<(b-a) / 2$, e sejam $f_{1} f_{2} \ldots f_{n} f_{n+1}$ as respectivas partes fracionárias ${ }^{2}$

2 A parte fracionária de um número real $r$ é o número real $r-k$, onde $k$ é o maior número inteiro tal que $k \leqslant r$. Claramente, a parte fracionária de $r$ é não negativa e é menor que 1. 
dos números da lista $g, 2 g, \ldots, n g,(n+1) g$. Como $g$ é irracional, temos $f_{i} \neq f_{j}(\forall i, j)$. Considerando a partição

$$
\left[0, \frac{1}{n}\right)_{\mathbb{R}},\left[\frac{1}{n}, \frac{2}{n}\right)_{\mathbb{R}}, \ldots,\left[\frac{n-2}{n}, \frac{n-1}{n}\right)_{\mathbb{R}},\left[\frac{n-1}{n}, 1\right)_{\mathbb{R}}
$$

do intervalo $[0,1)_{\mathbb{R}}$ em $n$ conjuntos, o Princípio da Casa dos Pombos $^{3}$ implica que existem $f_{i_{0}}$ e $f_{j_{0}}$ com $i_{0} \neq j_{0}$ tais que $f_{i_{0}}$ e $f_{j_{0}}$ pertencem a um mesmo intervalo dessa partição, resultando em $\left|f_{i_{0}}-f_{j_{0}}\right|<1 / n$. Assim, como cada $f_{i}$ é uma diferença entre um múltiplo de $g$ e um número inteiro, temos que existe um $z \in \mathbb{Z}(g)$ com $0<z<1 / n$. Seja $k$ o número inteiro tal que $k z \leqslant(a+b) / 2<(k+1) z$. Portanto, temos

$$
0 \leqslant \frac{a+b}{2}-k z<z<\frac{1}{n}<\frac{b-a}{2}
$$

resultando em $k z<(a+b) / 2<b$ e

$$
a=\frac{a+b}{2}-\frac{b-a}{2}<k z
$$

Resumindo, temos $a<k z<b$ e $k z \in \mathbb{Z}(g)$, provando que $\mathbb{Z}(g)$ é denso em $\mathbb{R}$.

A soma de dois elementos de $\mathbb{Z}(g)$ pertence a $\mathbb{Z}(g)$, e, para a maioria dos valores de $g \in I r r$, o produto de dois elementos de $\mathbb{Z}(g)$ não pertencerá necessariamente a $\mathbb{Z}(g)$, implicando que $\mathbb{Z}(g)$ não forma um subdomínio ordenado de $\mathbb{R}$ para esses valores de $g$.

Seja $n$ um número natural que não é um quadrado perfeito. Provaremos que $\mathbb{Z}(\sqrt{n})$ é um subdomínio ordenado próprio de $\mathbb{R}$. Temos

$$
\left(u_{1}+v_{1} \sqrt{n}\right) \cdot\left(u_{2}+v_{2} \sqrt{n}\right)=\left(u_{1} u_{2}+v_{1} v_{2} n\right)+\left(u_{1} v_{2}+u_{2} v_{1}\right) \sqrt{n} \in \mathbb{Z}(\sqrt{n})
$$

para quaisquer $u_{1}, u_{2}, v_{1}, v_{2} \in \mathbb{Z}$, resultando que $\mathbb{Z}(\sqrt{n})$ forma um subdomínio ordenado de $\mathbb{R}$. Seja $m$ um número natural que não é um quadrado perfeito e que não é um múltiplo de $n$. Suponha que $u$ e $v$ são números inteiros com $\sqrt{m}=u+v \sqrt{n}$. Se $v=0$, então $\sqrt{m}=u$ e $m=u^{2}$, o que é absurdo, e se $u=0$, então teremos $\sqrt{m}=v \sqrt{n}$ e $m=n v^{2}$, o que também é absurdo. Assim, $u$ e $v$ são não zero, e temos

$$
m=(\sqrt{m})^{2}=(u+v \sqrt{n})^{2}=u^{2}+n v^{2}+2 u v \sqrt{n}
$$

implicando que $\sqrt{n}$ é um número racional, o que é absurdo. Portanto, provamos que o número real $\sqrt{m}$ não pertence ao domínio ordenado $\mathbb{Z}(\sqrt{n})$.

Exemplo 1.6. Considere os domínios dos polinômios com coeficientes inteiros, racionais e reais, $\mathbb{Z}[x], \mathbb{Q}[x]$ e $\mathbb{R}[x]$ respectivamente. Para cada um desses três domínios, defina uma relação binária $<$ nesse domínio de modo que $p<q$ se, e somente se, o polinômio $q-p$ for

3 O Princípio da Casa dos Pombos (Teorema B.29) é o resultado de ZFC ou NBG cujo enunciado afirma que se um conjunto $A$ tiver cardinal maior que o cardinal de um conjunto $B$, então toda função sobrejetora do tipo $A \rightarrow B$ não será injetora. 
não zero e tiver coeficiente dominante positivo. Prova-se diretamente que cada relação < definida é uma ordem em cada domínio mencionado, e que as expansões desses domínios às estruturas de domínio ordenado correspondentes satisfazem aos axiomas (Inv) e (Pos). Portanto, os domínios $\mathbb{Z}[x], \mathbb{Q}[x]$ e $\mathbb{R}[x]$ são domínios ordenados quando munidos das respectivas ordens definidas.

Exemplo 1.7. Considere o conjunto $\mathcal{L} \subset \mathbb{Z} \mathbb{R}$ das famílias de reais $\left\{a_{n}\right\}_{n \in \mathbb{Z}}$ tais que

$$
a_{n}=0(\forall n<p)
$$

para algum número inteiro $p$. Informalmente, podemos dizer que $\mathcal{L}$ é o conjunto das famílias de reais com índices em $\mathbb{Z}$ que são eventualmente zero pela esquerda. Como toda família mencionada neste exemplo terá índices em $\mathbb{Z}$, omitiremos a informação " $n \in \mathbb{Z}$ " ao escrevermos tais famílias, sempre usando a letra $n$ como variável. Sejam $\left\{a_{n}\right\},\left\{b_{n}\right\} \in \mathcal{L}$. A operação de adição em $\mathcal{L}$ será definida por

$$
\left\{a_{n}\right\}+\left\{b_{n}\right\}:=\left\{a_{n}+b_{n}\right\} .
$$

A multiplicação em $\mathcal{L}$ será definida por

$$
\left\{a_{n}\right\} \cdot\left\{b_{n}\right\}:=\left\{c_{n}\right\}
$$

com

$$
c_{n}:= \begin{cases}0 & \text { se } n<p+q \\ \sum_{i=p}^{n-q} a_{i} b_{n-i} & \text { se } p+q \leqslant n,\end{cases}
$$

onde $p$ e $q$ são números inteiros tais que $a_{n}=0(\forall n<p)$ e $b_{n}=0(\forall n<q)$. O leitor deverá notar que o produto $\left\{a_{n}\right\} \cdot\left\{b_{n}\right\}$ definido acima independe das escolhas de $p$ e $q$.

A prova de que $\mathcal{L}$ é um domínio quando munido dessas operações é direta, onde:

- A família constante e igual a zero, $\{0\}$, é o elemento neutro da adição em $\mathcal{L}$, o qual será denotado simplesmente por 0 por abuso de linguagem;

- Para cada $r \in \mathbb{R}$ e para cada $m \in \mathbb{Z}$, denotaremos por $\llbracket r \rrbracket_{m}$ a família $\left\{u_{n}\right\}$ tal que $u_{m}=r$ e $u_{n}=0(\forall n \neq m)$. No caso $m=0$, denotaremos $\llbracket r \rrbracket_{0}$ simplesmente por $r$ por abuso de linguagem. A família $\llbracket 1 \rrbracket_{0}$, a qual é denotada simplesmente por 1 , é o elemento neutro da multiplicação em $\mathcal{L}$.

A ordem em $\mathcal{L}$ será definida de modo que $\left\{a_{n}\right\}<\left\{b_{n}\right\}$ se, e somente se, $\left\{a_{n}\right\} \neq\left\{b_{n}\right\}$ e $a_{k}<b_{k}$, onde $k$ é o menor número inteiro tal que $a_{k} \neq b_{k}$. Tal número inteiro $k$ sempre existe, pois caso contrário pelo menos uma das famílias $\left\{a_{n}\right\}$ e $\left\{b_{n}\right\}$ não seria 
eventualmente zero pela esquerda. A confirmação de que $<$ é uma ordem em $\mathcal{L}$ será deixada para o leitor.

Provaremos que $\mathcal{L}$ é um domínio ordenado quando munido dessa ordem. Se $\left\{a_{n}\right\},\left\{b_{n}\right\},\left\{c_{n}\right\} \in \mathcal{L} \operatorname{com}\left\{a_{n}\right\}<\left\{b_{n}\right\}$, e se $k$ for o menor número inteiro com $a_{k} \neq b_{k}$, então $a_{k}<b_{k}$, o número inteiro $k$ será o menor número inteiro tal que $a_{k}+c_{k} \neq b_{k}+c_{k}$, e, como $a_{k}+c_{k}<b_{k}+c_{k}$ (pois, como vimos, $\mathbb{R}$ é um domínio ordenado), teremos

$$
\left\{a_{n}\right\}+\left\{c_{n}\right\}=\left\{a_{n}+c_{n}\right\}<\left\{b_{n}+c_{n}\right\}=\left\{b_{n}\right\}+\left\{c_{n}\right\}
$$

provando que $\mathcal{L}$ satisfaz a (Inv). Se $0<\left\{a_{n}\right\}$ e $0<\left\{b_{n}\right\}$, onde $p$ e $q$ são os menores números inteiros tais que $0<a_{p}$ e $0<b_{q}$, então, denotando $\left\{c_{n}\right\}:=\left\{a_{n}\right\} \cdot\left\{b_{n}\right\}$, teremos $c_{n}=0(\forall n<p+q), \mathrm{e}$

$$
\begin{aligned}
c_{p+q} & =\sum_{i=p}^{(p+q)-q} a_{i} b_{(p+q)-i} \\
& =\sum_{i=p}^{p} a_{i} b_{p+q-i} \\
& =a_{p} b_{q} \\
& >0
\end{aligned}
$$

provando que $\mathcal{L}$ observa (Pos). Portanto, $\mathcal{L}$ é um domínio ordenado.

Denotaremos por $x$ a família $\llbracket 1 \rrbracket_{1}$. Diretamente da definição da multiplicação em $\mathcal{L}$, obtêm-se os seguintes fatos:

- $x^{-1}$ é a família $\llbracket 1 \rrbracket_{-1}$;

- $\left\{a_{n}\right\} \cdot x=\left\{a_{n-1}\right\}$ e $\left\{a_{n}\right\} \cdot x^{-1}=\left\{a_{n+1}\right\}$;

- Para $m \in \mathbb{Z}$, temos $\left\{a_{n}\right\} \cdot x^{m}=\left\{a_{n-m}\right\}$ e $x^{m}=\llbracket 1 \rrbracket_{m}$;

- Para $r \in \mathbb{R}$ e $m \in \mathbb{Z}$, temos $r \cdot \llbracket 1 \rrbracket_{m}=\llbracket r \rrbracket_{m}$.

Assim, temos

$$
\begin{aligned}
\left\{a_{n}\right\} & =\llbracket a_{p} \rrbracket_{p}+\llbracket a_{p+1} \rrbracket_{p+1}+\llbracket a_{p+2} \rrbracket_{p+2}+\cdots \\
& =a_{p} \cdot \llbracket 1 \rrbracket_{p}+a_{p+1} \cdot \llbracket 1 \rrbracket_{p+1}+a_{p+2} \cdot \llbracket 1 \rrbracket_{p+2}+\cdots \\
& =a_{p} \cdot x^{p}+a_{p+1} \cdot x^{p+1}+a_{p+2} \cdot x^{p+2}+\cdots \\
& =\sum_{n=p}^{\infty} a_{n} x^{n}
\end{aligned}
$$


onde $p$ é o menor número inteiro tal que $a_{p} \neq 0$. Note que $\sum_{n=p}^{\infty} a_{n} x^{n}$ não é uma soma ${ }^{4}$ - é meramente uma representação formal de $\left\{a_{n}\right\}$. Tais séries formais são conhecidas como séries de Laurent, ${ }^{5}$ e são cruciais no ramo da Análise Complexa. As operações de adição e multiplicação que definimos em $\mathcal{L}$ correspondem meramente às operações usuais de adição e multiplicação de polinômios na variável $x$, mas nesta ocasião em uma versão ampliada para "polinômios infinitos" que admitem uma quantidade finita de potências negativas de $x$.

A função $\psi: \mathbb{R} \rightarrow \mathcal{L}$ definida por $\psi(r):=\llbracket r \rrbracket_{0}$ é uma imersão (Definição A.42) entre domínios ordenados. Em vista disso, um elemento de $\mathcal{L}$ da forma $\llbracket r \rrbracket_{0} \operatorname{com} r \in \mathbb{R}$ é identificado com o número real $r$.

Antes de indicarmos as diferenças entre os variados tipos de domínios ordenados, é apropriado esclarecer as propriedades que todos eles têm em comum. As provas das afirmações do teorema a seguir estão devidamente explanadas em (14).

Teorema 1.8 (Propriedades dos Domínios Ordenados).

(a) Um elemento não zero $x$ de $D$ será positivo se, e somente se, o seu inverso aditivo, $-x$, for negativo. Analogamente, $x$ será negativo se, e somente se, o seu inverso aditivo for positivo;

(b) O produto de um elemento positivo e um elemento negativo de D será negativo;

(c) O produto de dois elementos negativos de D será positivo;

(d) Se $x<y, 0<z$, e $w<0$, então $x \cdot z<y \cdot z$ e $y \cdot w<x \cdot w$;

(e) Se $x$ for um elemento não zero de $D$, então $0<x^{2}$. Em particular, $0<1^{2}=1$;

(f) $D$ é infinito;

(g) Uma unidade ${ }^{6}$ em $D$ será positiva se, e somente se, o seu inverso multiplicativo, $x^{-1}$, for positivo. Analogamente, $x$ será negativo se, e somente se, o seu inverso multiplicativo for negativo.

Por (Lin), se $x \in D$, então $-x \leqslant x$ ou $x \leqslant-x$, implicando que o conjunto $\{-x, x\}$ tem um maior elemento. Tal máximo é dito ser o valor absoluto ou o módulo de $x \mathrm{e}$ é denotado por $|x|$. Se $|x|=0$, então $x=0$.

4 Não há preocupação alguma em relação à convergência de séries aqui. Trata-se apenas de uma decomposição da família $\left\{a_{n}\right\}$ em níveis.

5 Mais rigorosamente, as séries que representam os elementos de $\mathcal{L}$ são as séries de Laurent que não têm $x=0$ como uma singularidade essencial, ou seja, as séries centradas em 0 cujas partes principais têm uma quantidade finita de termos.

6 Um elemento $x$ de um anel é dito ser uma unidade ou ser inversível se existir um elemento $y$ desse anel tal que $x \cdot y=y \cdot x=1$. 
Exemplo 1.9. Não existe uma ordem $<$ em $\mathbb{C}$ que torna tal conjunto em um domínio ordenado. Isso ocorre pois $i \neq 0$ e $i^{2}=-1<0$, contradizendo a letra $(e)$ do Teorema 1.8.

\subsection{Corpos Ordenados}

Os domínios ordenados de maior importância na Matemática contêm apenas unidades, com a exceção do elemento 0. Essa propriedade adicional permite que a operação de divisão com denominadores não zero seja definida sobre a estrutura, e tal operação é imprescindível para a área da Análise, visto que as noções de derivada e integral de funções fundam-se peremptoriamente sobre a caracterização de limites de formas indeterminadas dos tipos $\%$ e $\infty / \infty$. A viabilidade de efetuar divisões também é bastante desejável do ponto de vista algébrico, uma vez que as soluções das equações polinômiais geralmente são expressas na forma de quocientes.

Definição 1.10. Um corpo ordenado é um domínio ordenado cujo domínio subentendido é um corpo. Um corpo não estritamente ordenado é um domínio não estritamente ordenado cujo domínio subentendido é um corpo.

A terminologia desenvolvida na Definição 1.1 para descrever as propriedades dos elementos dos domínios ordenados é usada para os corpos ordenados.

Sob a perspectiva da Teoria dos Modelos (Apêndice A), os corpos ordenados são as $L_{A O}$-estruturas que são modelos da $L_{A O}$-teoria gerada pelos axiomas $1-15$ discutidos na Seção 1.1 e pelo axioma

\section{(InvM) $(\forall x)(x \neq 0 \longrightarrow(\exists y) x \cdot y=1) . \quad$ (Invertibilidade Multiplicativa)}

Tal $L_{A O}$-teoria será denotada por $T_{C O}$ nesta dissertação.

Neste capítulo, assumiremos que $(K,+, \cdot, 0,1,<)$ é um corpo ordenado, o qual será denotado apenas por $K$ por simplicidade. O corpo subentendido em $K$ também será denotado por $K$ por abuso de linguagem. Denotaremos por $\div$ a operação de divisão em $K$, ou seja, a função $\div: K \times(K-\{0\}) \rightarrow K$ definida por $\div(x, y):=x / y$.

As noções de morfismo, imersão, imersão elementar e isomorfismo entre corpos ordenados são definidas como casos particulares das noções equivalentes referidas no estudo da Teoria dos Modelos (Seção A.15). Em particular, se $f: K \rightarrow L$ for um morfismo entre corpos ordenados, então a imagem de $1 \in K$ sobre $f$ será o elemento neutro multiplicativo de $L$, implicando em $f(q)=q(\forall q \in \mathbb{Q})$. A noção de subcorpo ordenado corresponde à noção de subestrutura (Definição A.41).

A ordem em um corpo ordenado é densa. Isso ocorre pois se $x$ e $y$ forem elementos de $K \operatorname{com} x<y$, então teremos

$$
x<\frac{x+y}{2}<y
$$


Exemplo 1.11. Se $f: K \rightarrow L$ for um morfismo entre corpos não estritamente ordenados, se $A$ for um subcorpo ordenado de $K$, e se $B$ for um subcorpo ordenado de $L$, então a imagem $f\langle A\rangle$ será um subcorpo ordenado de $L$, e a imagem inversa $f^{-1}\langle B\rangle$ será um subcorpo ordenado de $K$.

Exemplo 1.12. O domínio ordenado dos números inteiros, $\mathbb{Z}$, não é um corpo ordenado, e os domínios ordenados $\mathbb{Q}$ e $\mathbb{R}$ são corpos ordenados.

Exemplo 1.13. Seja $n$ um número natural que não é um quadrado perfeito. Se $u$ e $v$ forem números inteiros com $(u, v) \neq(0,0)$, então teremos

$$
\begin{aligned}
\frac{1}{u+v \sqrt{n}} & =\frac{1}{u+v \sqrt{n}} \cdot \frac{u-v \sqrt{n}}{u-v \sqrt{n}} \\
& =\frac{u-v \sqrt{n}}{u^{2}-n v^{2}} \\
& =\frac{u}{u^{2}-n v^{2}}-\frac{v}{u^{2}-n v^{2}} \cdot \sqrt{n}
\end{aligned}
$$

resultando que o número $u+v \sqrt{n}$ será inversível no domínio ordenado $\mathbb{Z}(\sqrt{n})$ (Exemplo 1.5) se, e somente se, o número inteiro não zero ${ }^{7} u^{2}-n v^{2}$ dividir $u$ e $v$. Caso tivermos $1<|u|$ e $v=0$, teremos que $u^{2}-n v^{2}=u^{2}$ não dividirá $u$, e o número $u+v \sqrt{n}=u$ não será inversível em $\mathbb{Z}(\sqrt{n})$. Portanto, $\mathbb{Z}(\sqrt{n})$ não é um corpo ordenado.

Exemplo 1.14. Os domínios ordenados $\mathbb{Z}[x], \mathbb{Q}[x]$ e $\mathbb{R}[x]$ (Exemplo 1.6) não são corpos ordenados, dado que nenhum polinômio não constante nesses domínios é uma unidade.

Exemplo 1.15. Como $\mathbb{R}[x]$ é um domínio, existe um corpo $\mathcal{R}(x)$ que contém $\mathbb{R}[x]$ como subanel tal que todo elemento $r(x)$ de $\mathcal{R}(x)$ pode ser escrito na forma $r(x)=p(x) / q(x)$ com $p(x), q(x) \in \mathbb{R}[x]$ e $q(x) \neq 0$. Tal corpo, o qual é o único com tais propriedades salvo isomorfismos, é dito ser o corpo das frações de $\mathbb{R}[x]$ ou o corpo das funções racionais. A ordem $<$ em $\mathcal{R}(x)$ será definida da seguinte maneira:

- Dizemos que $r(x)=p(x) / q(x) \in \mathcal{R}(x)$ é positivo se $0<p_{0} / q_{0}$, onde $p_{0}$ e $q_{0}$ são os coeficientes dominantes dos respectivos polinômios $p(x)$ e $q(x)$. Nesse caso, nota-se prontamente que o número real $p_{0} / q_{0}$ não depende da representação particular de $r(x)$ como uma fração da forma $p(x) / q(x) \operatorname{com} p(x), q(x) \in \mathbb{R}[x]$ e $q(x) \neq 0$;

- Para $r(x)$ e $s(x)$ em $\mathcal{R}(x)$, dizemos que $r(x)<s(x)$ se $s(x)-r(x)$ é positivo.

A relação < nitidamente satisfaz às condições (Irr) e (Lin). A prova de que $<$ observa (Trans) será deixada para o leitor.

Provaremos que $\mathcal{R}(x)$ é um corpo ordenado quando munido dessa ordem. A maneira que definimos a ordem $<$, viz. primeiramente definindo quando um elemento é $\overline{7}$ Se $u^{2}-n v^{2}=0$, então $\sqrt{n}= \pm u / v$ será um número racional, o que é absurdo. 
positivo e em seguida vinculando a ordem a uma diferença positiva, implica imediatamente que $\mathcal{R}(x)$ satisfaz a (Inv). Se $p(x) / q(x)$ e $p^{\prime}(x) / q^{\prime}(x)$ forem elementos positivos de $\mathcal{R}(x)$ com $p_{0}, p_{0}^{\prime}, q_{0}$ e $q_{0}^{\prime}$ sendo os coeficientes dominantes dos respectivos polinômios $p(x), p^{\prime}(x), q(x)$ e $q^{\prime}(x)$, então $0<p_{0} / q_{0}, 0<p_{0}^{\prime} / q_{0}^{\prime}$, e, como o quociente dos coeficientes dominantes do numerador e do denominador do produto

$$
\frac{p(x)}{q(x)} \cdot \frac{p^{\prime}(x)}{q^{\prime}(x)}=\frac{p(x) \cdot p^{\prime}(x)}{q(x) \cdot q^{\prime}(x)}
$$

é igual a

$$
\frac{p_{0} \cdot p_{0}^{\prime}}{q_{0} \cdot q_{0}^{\prime}}=\frac{p_{0}}{q_{0}} \cdot \frac{p_{0}^{\prime}}{q_{0}^{\prime}}>0
$$

temos $0<(p(x) / q(x)) \cdot\left(p^{\prime}(x) / q^{\prime}(x)\right)$, provando que $\mathcal{R}(x)$ satisfaz a (Pos) e é um corpo ordenado.

O leitor atento notará que a maneira mais rápida de verificar uma condição da forma $p / q<p^{\prime} / q^{\prime}$ consiste em manipular as frações de modo que os coeficientes dominantes de $q$ e $q^{\prime}$ sejam positivos, e em seguida investigar a condição equivalente $0<p^{\prime} q-p q^{\prime}$. Esta última será verdadeira se, e somente se, o coeficiente dominante do polinômio $p^{\prime} q-p q^{\prime}$ for positivo.

A função $\phi: \mathbb{R} \rightarrow \mathcal{R}(x)$ definida por $\phi(r):=r / 1$ (onde $r$ e 1 são polinômios constantes em $\mathbb{R}[x]$ ) é uma imersão (Definição A.42) entre corpos ordenados. Por esse motivo, um elemento de $\mathcal{R}(x)$ da forma $r / 1$ com $r \in \mathbb{R}$ é identificado com o número real $r$.

Exemplo 1.16. Sejam $\left\{a_{n}\right\}$ e $\left\{b_{n}\right\}$ dois elementos não zero do domínio ordenado $\mathcal{L}$ (Exemplo 1.7), e sejam $p$ e $q$ dois números tais que $p$ é o maior número inteiro com $a_{n}=0(\forall n<p)$ e $q$ é o maior número inteiro com $b_{n}=0(\forall n<q)$. Suponha que as famílias $\left\{a_{n}\right\}$ e $\left\{b_{n}\right\}$ são inversas uma da outra em $\mathcal{L}$. Temos $\left\{a_{n}\right\} \cdot\left\{b_{n}\right\}=1$, ou seja, $p+q=1, a_{p} b_{q}=1, \mathrm{e}$

$$
\sum_{i=p}^{n-q} a_{i} b_{n-i}=0(\forall n>p+q) .
$$

Portanto, temos $b_{q}=1 / a_{p}$, e, como

$$
\sum_{i=p}^{n-q} a_{i} b_{n-i}=a_{p} b_{n-p}+\sum_{i=p+1}^{n-q} a_{i} b_{n-i}=0(\forall n>p+q),
$$

substituindo $n$ por $m+p \operatorname{com} q<m$, temos

$$
a_{p} b_{m}+\sum_{i=p+1}^{m+p-q} a_{i} b_{m+p-i}=0(\forall m>q)
$$

resultando em

$$
b_{m}=\frac{-\sum_{i=p+1}^{m+p-q} a_{i} b_{m+p-i}}{a_{p}}(\forall m>q) .
$$


Note que se $p+1 \leqslant i$, então $p<i, p-i<0$, e $m+p-i<m$. Assim, para cada $m>q$, o valor de $b_{m}$ depende apenas dos valores da sequência finita $b_{q} b_{q+1} \ldots b_{m-2} b_{m-1}$, mostrando que a família $\left\{b_{n}\right\}$ é univocamente determinada pela família $\left\{a_{n}\right\}$. Isso prova que $\mathcal{L}$ é um corpo ordenado, e, por tal motivo, tal estrutura é chamada de corpo ordenado das séries de Laurent.

\subsection{A Propriedade Arquimediana}

Considere dois números naturais, $m$ e $n$, tais que $m<n$. Sabemos que $n \leqslant m n$, ou seja, temos

$$
n \leqslant \overbrace{m+m+\cdots+m}^{n \text { vezes }} .
$$

Em particular, existe uma soma de termos repetidos e iguais a $m$ que ultrapassa ou iguala o valor $n$. O mesmo ocorre para os números racionais positivos, visto que se $p / q<r / s$, com $p, q, r$ e $s$ números naturais, então, como $r q \leqslant s p \cdot r q$, teremos

$$
\frac{r}{s} \leqslant \frac{p}{q} \cdot r q=\overbrace{\frac{p}{q}+\frac{p}{q}+\cdots+\frac{p}{q}}^{r q \text { vezes }} .
$$

No entanto, isso não ocorre para todo domínio ordenado. Considere o corpo ordenado das funções racionais, $\mathcal{R}(x)$, onde temos $0<x^{-1}<1$. Nesse cenário, temos

$$
\overbrace{x^{-1}+x^{-1}+\cdots+x^{-1}}^{n \text { vezes }}=n x^{-1}<1(\forall n \in \mathbb{N})
$$

ou seja, independentemente de quantas vezes o "valor" $x^{-1}$ for acumulado, o resultado da soma nunca ultrapassará o elemento neutro multiplicativo do corpo. Isso indica que os elementos $x^{-1}$ e 1 estão em classes essencialmente distintas de elementos de $\mathcal{R}(x)$.

Definição 1.17. Sejam $x$ e $y$ dois elementos de $D$.

(a) Dizemos que $x$ é infinitesimal em relação a $y$ ( ou $y$ é infinito em relação a $x)$ se $n|x|<|y|(\forall n \in \mathbb{N})$;

(b) Dizemos que $x$ é infinitesimal se $x$ é infinitesimal em relação a 1, ou seja, se $n|x|<1(\forall n \in \mathbb{N})$. Se $S$ é um subconjunto de $D$, então o conjunto de todos os elementos infinitesimais de $S$ é denotado por $\operatorname{Inf}(S)$;

(c) Dizemos que $x$ é finito se $x$ não é infinito em relação a 1, ou seja, se $|x| \leqslant n(\exists n \in \mathbb{N})$. Se $S$ é um subconjunto de $D$, então o conjunto de todos os elementos finitos de $S$ é denotado por Fin $(S)$;

(d) Dizemos que $x$ é infinito se $x$ não é finito, ou seja, se $n<|x|(\forall n \in \mathbb{N})$. Se $S$ é um subconjunto de $D$, então o conjunto de todos os elementos infinitos de $S$ é denotado por $S_{\infty}$; 
(e) Dizemos que $x$ é apreciável se $x$ é finito e não é infinitesimal, ou seja, se $|x| \leqslant n(\exists n \in \mathbb{N})$ e $1 \leqslant n|x|(\exists n \in \mathbb{N})$.

O elemento neutro aditivo, 0, é infinitesimal em relação a qualquer elemento não zero de $D$. Todo infinitesimal é finito, e todo número racional em $D$ é apreciável. Uma unidade $x$ será infinitesimal se, e somente se, $x^{-1}$ for infinito.

Teorema 1.18. Sejam $x, y \in D$. A Tabela 1 a seguir sumariza a situação da soma $x+y$, do produto $x y$ e do quociente $x / y$ (caso y for uma unidade) para diversas combinações de atributos de $x$ e y descritos na Definição $1.1 \%$.

Tabela 1 - Operações aritméticas entre elementos infinitesimais, finitos, infinitos e apreciáveis

\begin{tabular}{|c|c|c|c|c|}
\hline \multicolumn{1}{|c|}{$x$} & $y$ & $x+y$ & $x y$ & $x / y$ \\
\hline \hline Infinitesimal & Infinitesimal & Infinitesimal & Infinitesimal & Indeterminado \\
\hline Infinitesimal & Finito & Finito & Infinitesimal & Indeterminado \\
\hline Infinitesimal & Infinito & Infinito & Indeterminado & Infinitesimal \\
\hline Infinitesimal & Apreciável & Apreciável & Infinitesimal & Infinitesimal \\
\hline Finito & Infinitesimal & Finito & Infinitesimal & Indeterminado \\
\hline Finito & Finito & Finito & Finito & Indeterminado \\
\hline Finito & Infinito & Infinito & Indeterminado & Infinitesimal \\
\hline Finito & Apreciável & Apreciável & Finito & Finito \\
\hline Infinito & Infinitesimal & Infinito & Indeterminado & Infinito \\
\hline Infinito & Finito & Infinito & Indeterminado & Infinito \\
\hline Infinito & Infinito & Infinito & Infinito & Indeterminado \\
\hline Infinito & Apreciável & Infinito & Infinito & Infinito \\
\hline Apreciável & Infinitesimal & Apreciável & Infinitesimal & Infinito \\
\hline Apreciável & Finito & Apreciável & Finito & Indeterminado \\
\hline Apreciável & Infinito & Infinito & Infinito & Infinitesimal \\
\hline Apreciável & Apreciável & Apreciável & Apreciável & Apreciável \\
\hline
\end{tabular}

A entrada "indeterminado" na Tabela 1 representa que o resultado da operação indicada não será necessariamente infinitesimal, finito, infinito ou apreciável.

Demonstração. Trataremos apenas das duas seguintes hipóteses:

1. Se $x$ e $y$ forem infinitesimais, então $x+y$ será infinitesimal;

2. Se $x$ for infinitesimal e $y$ for finito, então $x y$ será infinitesimal.

As provas dos casos restantes mostrados na Tabela 1 são diretas e serão deixadas para o leitor. 
1. Se $x$ e $y$ forem dois infinitesimais em $D$, e se $n \in \mathbb{N}$, então

$$
\begin{aligned}
n|x+y| & \leqslant n|x|+n|y| \\
& <1+1 \\
& =2 .
\end{aligned}
$$

Nesse caso, escolhendo $n=2 m$ com $m \in \mathbb{N}$, temos $2 m|x+y|<2$, resultando em

$$
m|x+y|<1(\forall m \in \mathbb{N}),
$$

e provando que $x+y$ é infinitesimal.

2. Se $x$ for infinitesimal, se $y$ for finito, e se $n \in \mathbb{N}$, então existirá um $m \in \mathbb{N}$ com $|y| \leqslant m$, e teremos

$$
\begin{aligned}
n|x y| & =n \cdot|x| \cdot|y| \\
& \leqslant n \cdot|x| \cdot m \\
& =m n|x| \\
& <1,
\end{aligned}
$$

mostrando que $x y$ é infinitesimal.

Se $x$ e $y$ forem elementos não zero de $D$ tais que $x$ é infinitesimal e $y$ não é infinitesimal, então $x$ será infinitesimal em relação a $y$. Isso ocorre pois $y$ será apreciável ou infinito, e em ambos os casos teremos que $y / x$ será infinito, ou seja, teremos

$$
n<\left|\frac{y}{x}\right|=\frac{|y|}{|x|}(\forall n \in \mathbb{N})
$$

resultando em $n|x|<|y|(\forall n \in \mathbb{N})$. Em particular, teremos $|x|<|y|$.

\section{Corolário 1.19.}

(a) Fin $(D)$ é um subdominio ordenado de $D$;

(b) $\operatorname{Inf}(D)$ é um subdominio ordenado sem unidade de $D$ e é um ideal em Fin $(D)$.

Definição 1.20. Dizemos que $D$ é um domínio arquimediano ${ }^{9}$ se não existem $x, y \in D$ não zero tais que $x$ é infinitesimal em relação a $y$. Caso contrário, dizemos que $D$ é um domínio não arquimediano.

8 Um domínio ordenado sem unidade é uma $L_{A}$-estrutura (Exemplo A.9) que é modelo dos axiomas dos domínios ordenados exceto os axioma 6 e 10 descritos na Seção 1.1.

9 Em 1882 (1), o matemático austríaco Otto Stolz (1842-1905) vinculou o nome do matemático grego Arquimedes de Siracusa a tal propriedade, uma vez que ela aparece como Axioma V na obra deste entitulada Sobre a esfera e o cilíndro. 
A condição que define os domínios arquimedianos é conhecida como Propriedade Arquimediana.

Se $D$ for arquimediano, então teremos $\operatorname{Inf}(D)=\{0\}, \operatorname{Fin}(D)=D$, e $D_{\infty}=\emptyset$.

Exemplo 1.21. O domínio ordenado dos números inteiros, $\mathbb{Z}$, e o corpo ordenado dos números racionais, $\mathbb{Q}$, são arquimedianos. Utilizando o fato de que $\mathbb{Q}$ é denso em $\mathbb{R}$, prova-se que $\mathbb{R}$ também é um corpo arquimediano.

Exemplo 1.22. Se $n$ for um número natural que não é um quadrado perfeito, então o domínio ordenado $\mathbb{Z}(\sqrt{n})$ será arquimediano, visto que ele é um subdomínio ordenado de $\mathbb{R}$.

Exemplo 1.23. Os domínios ordenados $\mathbb{Z}[x], \mathbb{Q}[x]$ e $\mathbb{R}[x]$ (Exemplo 1.6) são não arquimedianos, uma vez que temos $n<x(\forall n \in \mathbb{N})$ para cada um deles. Se $m$ e $n$ forem números inteiros não negativos com $m<n$, então $x^{m}$ será infinitesimal em relação a $x^{n}$. Percebe-se que

$$
\begin{aligned}
& \operatorname{Inf}(\mathbb{R}[x])=\{0\}, \\
& \operatorname{Fin}(\mathbb{R}[x])=\mathbb{R},
\end{aligned}
$$

e

$$
\mathbb{R}[x]_{\infty}=\mathbb{R}[x]-\mathbb{R}
$$

e equações análogas são válidas para $\mathbb{Z}[x]$ e $\mathbb{Q}[x]$.

Exemplo 1.24. O corpo ordenado das funções racionais, $\mathcal{R}(x)$ (Exemplo 1.15), é não arquimediano. Se $m<n$ forem números inteiros, então $x^{m}$ será infinitesimal em relação a $x^{n}$. Em particular, se $n$ for um número natural, então $x^{-n}$ será infinitesimal e $x^{n}$ será infinito. Constata-se diretamente que

$$
\begin{gathered}
\operatorname{Inf}(\mathcal{R}(x))=\left\{\frac{p}{q} \vdots p, q \in \mathbb{R}[x] \text { com } q \neq 0 \text { e gr }(p)<\operatorname{gr}(q)\right\}, \\
\operatorname{Fin}(\mathcal{R}(x))=\left\{\frac{p}{q} \vdots p, q \in \mathbb{R}[x] \operatorname{com} q \neq 0 \text { e } \operatorname{gr}(p) \leqslant \operatorname{gr}(q)\right\},
\end{gathered}
$$

e

$$
\mathcal{R}(x)_{\infty}=\left\{\frac{p}{q}: p, q \in \mathbb{R}[x] \operatorname{com} q \neq 0 \text { e } \operatorname{gr}(q)<\operatorname{gr}(p)\right\} .
$$

Exemplo 1.25. O corpo ordenado das séries de Laurent, $\mathcal{L}$ (Exemplo 1.7), é não arquimediano. Se $m<n$ forem números inteiros, então $x^{n}$ será infinitesimal em relação a $x^{m}$. Em particular, se $n$ for um número natural, então $x^{-n}$ será infinito e $x^{n}$ será infinitesimal. Constata-se diretamente que

$$
\operatorname{Inf}(\mathcal{L})=\left\{\left\{a_{n}\right\} \vdots a_{i}=0(\forall i \leqslant 0)\right\},
$$




$$
\operatorname{Fin}(\mathcal{L})=\left\{\left\{a_{n}\right\} \vdots a_{i}=0(\forall i<0)\right\}
$$

$\mathrm{e}$

$$
\mathcal{L}_{\infty}=\left\{\left\{a_{n}\right\} \vdots a_{i} \neq 0(\exists i<0)\right\} .
$$

Teorema 1.26. As seguintes condições são equivalentes:

(a) $K$ é arquimediano;

(b) K não tem um elemento infinitesimal não zero;

(c) K não tem um elemento infinito.

Demonstração. As implicações $(a) \Rightarrow(b)$ e $(b) \Rightarrow(c)$ são imediatas.

$(c) \Rightarrow(a)$ : Suponha que existem $x, y \in K$ não zero tais que $x$ é infinitesimal em relação a $y$, ou seja, tais que

$$
n|x|<|y|(\forall n \in \mathbb{N}) .
$$

Como $x$ é não zero, temos que $|x|$ é invertível, e, multiplicando ambos os lados daquela inequação por $|x|^{-1}$, temos

$$
n<|y| \cdot|x|^{-1}(\forall n \in \mathbb{N}) \text {, }
$$

implicando que $|y| \cdot|x|^{-1}$ é infinito, e contradizendo (c).

\subsection{Relações Confrontantes em Domínios Ordenados}

Nesta seção, trataremos de uma classe de relações de equivalência $\equiv$ em $D$ que induzem canonicamente uma ordem no quociente $D / \equiv$.

Definição 1.27. Uma relação de equivalência $\equiv$ em $D$ é dita ser confrontante em $D$ se as seguintes condições são observadas:

(Som) Se $x \equiv y$, então $x+z \equiv y+z$;

(Conf) $x<y<z$ e $x \equiv z$ implicam $x \equiv y \equiv z$.

Exemplo 1.28. Considere a relação binária $\triangleq \mathrm{em} \mathbb{Q}[x]$ (Exemplo 1.6) definida por

$$
\begin{aligned}
\sum_{i=0}^{r} c_{i} x^{i} \triangleq \sum_{i=0}^{s} d_{i} x^{i}: & \Leftrightarrow c_{r} x^{r}=d_{s} x^{s} \\
& \Leftrightarrow\left(r=s \text { e } c_{r}=d_{s}\right) .
\end{aligned}
$$


Verifica-se prontamente que $\triangleq$ é uma relação de equivalência em $\mathbb{Q}[x]$ que obedece a (Som). Sejam $p(x)=\sum_{i=0}^{l} c_{i} x^{i}, q(x)=\sum_{i=0}^{m} d_{i} x^{i}$ e $r(x)=\sum_{i=0}^{n} e_{i} x^{i}$ três polinômios em $\mathbb{Q}[x]$ $\operatorname{com} p(x)<q(x)<r(x)$ e $p(x) \triangleq r(x)$. Assim, teremos $l=n$ e $c_{l}=e_{n}=e_{l}$. Se $l<m$, então os graus dos polinômios $q(x)-p(x)$ e $r(x)-q(x)$ serão iguais a $m$, e teremos $0<d_{m}<0$, o que é absurdo. Se $m<l$, então os graus dos polinômios $q(x)-p(x)$ e $r(x)-q(x)$ serão iguais a $l$, e teremos $c_{l}<0<e_{l}=c_{l}$, o que é absurdo. Portanto, temos $l=m=n$, resultando em $c_{l} \leqslant d_{l} \leqslant e_{l}=c_{l}, c_{l}=d_{l}=e_{l}$, e $p(x) \triangleq r(x)$. Isso prova que $\triangleq$ satisfaz a (Conf) e é confrontante em $\mathbb{Q}[x]$.

Na Seção 1.6, lidaremos com os dois exemplos de relações confrontantes em $D$ de maior influência para a teoria dos domínios ordenados.

Nesta seção, consideraremos uma relação de equivalência $\equiv$ em $D$ que é confrontante em $D$, e, para cada $x \in D$, a classe de equivalência de $x$ em relação a $\equiv$ será denotada por $\bar{x}$.

Seja $\sqsubset$ a relação binária no quociente $D / \equiv$ dada por

$$
A \sqsubset B: \Leftrightarrow(\forall x \in A)(\forall y \in B) x<y
$$

A proposição a seguir caracteriza a versão não estrita dessa relação.

Proposição 1.29. Se $A$ e $B$ forem classes de equivalência em $D / \equiv$, então

$$
(A \sqsubset B \text { ou } A=B) \Leftrightarrow(\exists x \in A)(\exists y \in B) x \leqslant y .
$$

Demonstração. A prova da parte $\Rightarrow$ da condição é imediata. Suponha que $x \in \bar{a}=A$, $y \in \bar{b}=B$, e $a \leqslant b$. Assim, temos $x \equiv a$ e $b \equiv y$. Por (Som), temos $x-a \equiv 0, b-y \equiv 0$, e

$$
(x-a)+(b-y) \equiv 0+(b-y)=b-y \equiv 0 .
$$

Se $y \leqslant x$, então

$$
0 \leqslant x-y=(x-a)+(a-y) \leqslant(x-a)+(b-y) \equiv 0
$$

e por (Conf) teremos $x-y \equiv 0$, resultando em $A=B$. Portanto, se $A \neq B$, então por (Lin) teremos $x<y$ para quaisquer $x \in A$ e $y \in B$.

Corolário 1.30. A relação $\sqsubset$ é uma ordem em D/ $\equiv$.

Definição 1.31. O quociente $D / \equiv$ munido da ordem $\sqsubset$ é dito ser o conjunto ordenado quociente de $D$ módulo $\equiv$, e ele também é denotado por $D / \equiv$ por abuso de linguagem. 


\subsection{Ideais Ordenados}

Constata-se na Teoria dos Anéis que qualquer ideal $I$ no domínio subentendido em $D$ induz sobre o quociente $D / I$ uma estrutura de domínio. Porém, para que possamos definir uma estrutura canônica de domínio ordenado sobre aquele quociente, o ideal $I$ deve satisfazer a uma propriedade suplementar às que qualquer ideal observa.

Definição 1.32. Um subconjunto $I$ de $D$ é dito ser um ideal ordenado em $D$ se $I$ for um ideal no domínio subentendido em $D$ tal que as condições $x \in D, y \in I$ e $0<x<y$ implicam $x \in I$.

Exemplo 1.33. O subconjunto $\{0\}$ de $D$, o qual é denotado por 0 , é um ideal ordenado em $D$.

Exemplo 1.34. Como vimos no Corolário 1.19, o conjunto Inf $(D)$ é um ideal em Fin $(D)$. Se $x \in \operatorname{Fin}(D), y \in \operatorname{Inf}(D)$, e $0<x<y$, então $x$ será infinitesimal, pois caso contrário existirá um número natural $n$ com $1<n x<n y$, contradizendo a circunstância de que $y$ é infinitesimal. Assim, Inf $(D)$ é um ideal ordenado em Fin $(D)$.

Exemplo 1.35. Se $f: D \rightarrow E$ for um morfismo entre domínios não estritamente ordenados, então ker $(f)$ será um ideal ordenado em $D$.

Exemplo 1.36. Seja $\equiv$ uma relação de equivalência em $D$ que é confrontante em $D$, e seja $I$ a classe de equivalência de 0 em relação a $\equiv$. A estrutura de $D$ induz sobre $I$ uma estrutura de grupo comutativo $(I,+)$, e para quaisquer $x, y \in D$ as condições $0<x<y$ e $y \in I$ implicam $x \in I$. Contudo, como $I$ não é necessariamente fechado sob a multiplicação, não pode-se afirmar que $I$ é um ideal ordenado em $D$. Se para quaisquer $x, y \in D$ a condição $x \equiv 0$ implicar na condição $x y \equiv 0$, o conjunto $I$ será um ideal ordenado em $D$. Veremos que a noção de relação de equivalência confrontante generaliza a noção de ideal ordenado (Teorema 1.37).

Nesta seção, consideraremos um ideal ordenado $I$ em $D$. Como $I$ é um ideal no domínio subentendido em $D$, a relação binária $\equiv$ em $D$ definida por

$$
x \equiv y: \Leftrightarrow x-y \in I
$$

é uma relação de equivalência em $D$. Para cada $x \in D$, a classe de equivalência de $x$ em relação a $\equiv$ é igual ao conjunto $x+I$, o qual será denotado por $\bar{x}$ nesta seção.

Define-se o domínio quociente de $D$ módulo $I$, denotado por $D / I$, como sendo o conjunto

$$
D / \equiv=\{\bar{x}: x \in D\}
$$

munido das operações

$$
\bar{x}+\bar{y}:=\overline{x+y}
$$




$$
\bar{x} \cdot \bar{y}:=\overline{x \cdot y}
$$

Prova-se que tais operações são bem definidas e que $D / I$ é um domínio (33).

Teorema 1.37. A relação de equivalência $\equiv$ em $D$ é confrontante em $D$.

Demonstração. Se $x<y<z \operatorname{com} x \equiv z$, então $0<y-x<z-x$ e $z-x \in I$, implicando em $y-x \in I$ e $z \equiv x \equiv y$.

Seja $\sqsubset$ a ordem do conjunto ordenado quociente de $D$ módulo $\equiv$ (Corolário 1.30).

Teorema 1.38. O dominio quociente D/I é um dominio ordenado quando munido da ordem $\sqsubset$.

Demonstração. Sejam $\bar{x}, \bar{y}, \bar{z} \in D / I$. Se $\bar{x} \sqsubset \bar{y}$, então $x<y, x+z<y+z$, e

$$
\bar{x}+\bar{z}=\overline{x+z} \sqsubseteq \overline{y+z}=\bar{y}+\bar{z}
$$

resultando claramente em $\bar{x}+\bar{z} \sqsubset \bar{y}+\bar{z}$, e provando que $D / I$ observa (Inv). Se $\overline{0} \sqsubset \bar{x}$ e $\overline{0} \sqsubset \bar{y}$, então $0<x, 0<y, 0<x \cdot y$, e

$$
\overline{0} \sqsubseteq \overline{x \cdot y}=\bar{x} \cdot \bar{y}
$$

resultando em $\overline{0} \sqsubset \bar{x} \cdot \bar{y}$ pois $D / I$ não tem divisores de zero, e provando que $D / I$ satisfaz a (Pos).

Definição 1.39. O domínio quociente $D / I$ munido da ordem $\sqsubset$ é dito ser o domínio ordenado quociente de $D$ módulo $I$, e ele também é denotado por $D / I$ por abuso de linguagem.

A demonstração do teorema a seguir será deixada para o leitor.

Teorema 1.40 (Primeiro Teorema do Isomorfismo para Domínios Não Estritamente Ordenados). Se $f: D \rightarrow E$ for um morfismo entre domínios não estritamente ordenados, então a função $g: D / \operatorname{ker}(f) \rightarrow \operatorname{Im}(f)$ dada por

$$
g(x+\operatorname{ker}(f)):=f(x)
$$

será bem definida e será um isomorfismo entre domínios ordenados. 


\subsection{Mônadas e Galáxias}

Como 0 é infinitesimal, como toda soma de infinitesimais é infinitesimal, e como toda soma de finitos é finita, podemos definir duas relações de equivalência em $D$ como segue.

\section{Definição 1.41 .}

- Denotaremos por $\sim_{D}$ a relação binária em $D$ definida de modo que $x \sim_{D} y$ se, e somente se, $x-y$ for infinitesimal. Tal relação é uma relação de equivalência, e ela é chamada de relação de proximidade infinita. Quando $x$ e $y$ forem elementos de $D$ tais que $x \sim_{D} y$, dizemos que $x$ e $y$ estão infinitamente próximos. Quando o domínio ordenado $D$ estiver implícito no contexto, denotaremos a relação $\sim_{D}$ simplesmente por $\sim$. Para cada $x \in D$, denotaremos por $\bar{x}$ a classe de equivalência ${ }^{10}$ de $x$ em relação a $\sim$, a qual é dita ser a mônada de $x$.

- Denotaremos por $\approx_{D}$ a relação binária em $D$ definida de modo que $x \approx_{D} y$ se, e somente se, $x-y$ for finito. Tal relação é uma relação de equivalência, e ela é chamada de relação de proximidade finita. Quando $x$ e $y$ forem elementos de $D$ tais que $x \approx_{D} y$, dizemos que $x$ e $y$ estão finitamente próximos. Quando o domínio ordenado $D$ estiver implícito no contexto, denotaremos a relação $\approx_{D}$ simplesmente por $\approx$. Para cada $x \in D$, denotaremos por $\mathcal{G}(x)$ a classe de equivalência de $x$ em relação a $\approx$, a qual é dita ser a galáxia de $x$.

Temos $\operatorname{Inf}(D)=\overline{0}$ e $\operatorname{Fin}(D)=\mathcal{G}(0)$, e se $x \in D$, então teremos $\bar{x}=x+\overline{0}$ e $\mathcal{G}(x)=x+\mathcal{G}(0)$. Portanto, para caracterizar as mônadas e as galáxias em $D$ basta descrever os conjuntos $\overline{0}$ e $\mathcal{G}(0)$. Toda mônada é isomorfa a $\overline{0}$ como conjuntos ordenados, e toda galáxia é isomorfa a $\mathcal{G}(0)$ como conjuntos ordenados.

Em termos intuitivos, pode-se pensar em uma mônada $\bar{x}$ como um envólucro ao redor de $x$ de comprimento pequeno e insignificante, e pode-se pensar em uma galáxia $\mathcal{G}(x)$ como uma extensa região contendo $x$ limitada apenas pela ressalva de que a distância entre quaisquer dois pontos nessa região deve ser finita.

\section{Corolário 1.42 .}

(a) $\operatorname{Inf}(D)$ é um ideal ordenado em Fin $(D)$;

(b) $\operatorname{Inf}(K)$ é um ideal maximal em Fin $(K)$.

Demonstração. Basta provar a maximalidade do ideal $\operatorname{Inf}(K)$ em Fin $(K)$. Suponha que $I$ é um ideal em Fin $(K)$ que contém $\operatorname{Inf}(K)$ propriamente. Assim, existe um $x \in I$

\footnotetext{
10 Muitos autores denotam essa classe de equivalência por $\mu(x)$. Adotarei a notação algébrica padrão.
} 
com $x \notin \operatorname{Inf}(K)$. Como $0 \in \operatorname{Inf}(K)$, temos $x \neq 0$. Se $x^{-1}$ for infinito, então $x$ será infinitesimal, o que é absurdo. Portanto, temos $x^{-1} \in$ Fin $(K)$, ou seja, $x$ é uma unidade em Fin $(K)$, implicando em $I=$ Fin $(K)$.

A letra (b) do Corolário 1.42 implica que o quociente Fin $(K) / \operatorname{Inf}(K)$ entre domínios ordenados é um corpo ordenado.

Corolário 1.43. As relações de equivalência $\sim e \approx e m D$ são confrontantes em $D$.

Nesta dissertação, denotaremos as respectivas ordens dos conjuntos ordenados quocientes $D / \sim \mathrm{e} D / \approx$ por $\prec \mathrm{e} \triangleleft$. Tais ordens são densas quando lidamos com corpos ordenados, como mostra a proposição a seguir.

\section{Proposição 1.44.}

(a) A relação $\prec$ é uma ordem densa em $K / \sim$;

(b) A relação $\triangleleft$ é uma ordem densa em $K / \approx$.

Demonstração.

(a) Suponha que $a$ e $b$ são elementos de $K$ tais que $\bar{a} \prec \bar{b}$. Como $a \not b$, existe um número natural $n$ tal que $\frac{1}{2 n}<\frac{1}{n}<b-a$, e temos

$$
a<a+\frac{1}{2 n}<b
$$

Claramente, temos $a \not x a+\frac{1}{2 n}$, e se $a+\frac{1}{2 n} \sim b$, então

$$
\frac{1}{2 n}=\frac{1}{n}-\frac{1}{2 n}<b-a-\frac{1}{2 n} \sim 0,
$$

o que é absurdo. Portanto, temos

$$
\bar{a} \prec \overline{a+\frac{1}{2 n}} \prec \bar{b} .
$$

(b) Suponha que $a$ e $b$ são elementos de $K$ tais que $\mathcal{G}(a) \triangleleft \mathcal{G}(b)$. Como $a \not z b$, temos $n<b-a(\forall n \in \mathbb{N})$, e, tomando $n=2 m$, temos $m<(b-a) / 2(\forall m \in \mathbb{N})$, ou seja, $(b-a) / 2$ é infinito. Assim, temos

$$
a<a+\frac{b-a}{2}<b,
$$

e claramente temos $a \not \approx a+{ }^{(b-a) / 2} \not \approx b$, resultando em

$$
\mathcal{G}(a) \triangleleft \mathcal{G}\left(a+\frac{b-a}{2}\right) \triangleleft \mathcal{G}(b) .
$$




\subsection{A Propriedade do Supremo}

Os conceitos referidos na definição a seguir são indispensáveis para o estudo das ordens.

Definição 1.45. Sejam $E \subset D$ e $y \in D$.

- Dizemos que y é uma cota superior (resp. cota inferior) de $E$ se $x \leqslant y$ (resp. $y \leqslant x)$ para todo $x \in E$. Nesse caso, dizemos que $y$ é o supremo ou a menor cota superior (resp. ínfimo ou a maior cota inferior) de $E$ se $y \leqslant z$ (resp. $z \leqslant y$ ) para qualquer cota superior (resp. cota inferior) $z$ de $E$. Caso o supremo (resp. ínfimo) de $E$ exista, ele é denotado por sup $(E)($ resp. inf $(E))$;

- Dizemos que y é um maior elemento (resp. menor elemento) de $E$ se $y \in E$ e $y$ é uma cota superior (resp. cota inferior) de $E$.

Se $E$ tiver um maior elemento (resp. menor elemento), então ele será um supremo (resp. ínfimo) de $E$, e se $E$ tiver um supremo (resp. ínfimo), então ele será único.

Considere o conjunto

$$
R:=\left\{x \in D \mid x^{2}<2\right\}
$$

Tal conjunto é não nulo (pois $1 \in R$ ) e o elemento $2 \in D$ é uma cota superior de $R$, já que se $x \in R$ e $2<x$, então

$$
4=2^{2}=2 \cdot 2<x \cdot x=x^{2}<2,
$$

o que é absurdo, resultando em $x<2(\forall x \in R)$. A existência de uma cota superior de $R$ acarreta na existência de uma menor cota superior de $R$ ? Isto é, o conjunto de todas as cotas superiores de $R$ tem um menor elemento? A resposta é positiva para o corpo ordenado dos números reais $\left(\operatorname{com} \sup _{\mathbb{R}}(R)=\sqrt{2}\right)$, e, como veremos na Seção 1.9, essa peculiaridade define $\mathbb{R}$ salvo isomorfismos entre corpos ordenados.

Definição 1.46. Dizemos que um domínio ordenado $D$ satisfaz à Propriedade do Supremo se todo subconjunto não nulo de $D$ que tem uma cota superior tem um supremo em $D$.

Na Definição 1.46, podemos substituir os vocábulos "cota superior" e "supremo" respectivamente por "cota inferior" e "ínfimo" sem alterar a essência da definição, uma vez que se $y$ for uma cota inferior de um subconjunto $E$ de $D$, então $-y$ será uma cota superior do conjunto $\{-x: x \in E\}$.

Proposição 1.47. Se D obedecer à Propriedade do Supremo, então D será arquimediano. 
Demonstração. Suponha que D obedece à Propriedade do Supremo e é não arquimediano. Seja $X$ o conjunto

$$
X:=\{x \in D: 0 \leqslant x \text { e } x \text { é finito }\} .
$$

Como $0 \in X$, tal conjunto é não nulo, e, como qualquer elemento infinito positivo de $D$ é uma cota superior de $X$, temos que $D$ é limitado superiormente (Teorema 1.26, Letra (c)) e existe um supremo $s$ de $X$ em $D$. Se $s \in X$, então $s+1 \in X$, o que é absurdo visto que $s<s+1$, e se $s \notin X$, então $s-1$ será infinito e será uma cota superior de $X$ em $D$ com $s-1<s$, o que também é absurdo. Portanto, a suposição inicial da demonstração é falsa.

Proposição 1.48. Se D for um subdominio ordenado próprio de um domínio ordenado $E$, e se $D$ for denso em E, então D não obedecerá à Propriedade do Supremo.

Demonstração. Se $x \in E-D$, então, como $x<x+1$ e como $D$ é denso em $E$, o conjunto

$$
\{y \in D \vdots y<x\}
$$

terá uma cota superior mas não terá um supremo em $D$.

Exemplo 1.49. O domínio ordenado dos números inteiros, $\mathbb{Z}$, satisfaz à Propriedade do Supremo.

Exemplo 1.50. O corpo ordenado dos números racionais, $\mathbb{Q}$, não satisfaz à Propriedade do Supremo, visto que $\mathbb{Q}$ é denso em $\mathbb{R}$.

Exemplo 1.51. O corpo ordenado dos números reais, $\mathbb{R}$, satisfaz à Propriedade do Supremo. Para provar esse fato, deve-se trabalhar com uma construção particular de $\mathbb{R}$ como as construções por sequências de Cauchy e por cortes de Dedekind. Tais construções são desenvolvidas com detalhe em (14). Na Seção 2.8, veremos outra maneira de definir $\mathbb{R}$ a partir do corpo ordenado dos números racionais, $\mathbb{Q}$.

Exemplo 1.52. Se $n$ for um número natural que não é um quadrado perfeito, então o domínio ordenado $\mathbb{Z}(\sqrt{n})$ (Exemplo 1.5) não obedecerá à Propriedade do Supremo, visto que $\mathbb{Z}(\sqrt{n})$ é denso em $\mathbb{R}$ (Proposição 1.48).

Exemplo 1.53. Os domínios ordenados não arquimedianos $\mathbb{Q}[x]$ e $\mathbb{R}[x]$ (Exemplo 1.6) não obedecem à Propriedade do Supremo. Se o subconjunto $\mathbb{Z}$ de $\mathbb{Z}[x]$ tiver um supremo, então nota-se facilmente que esse supremo será positivo e terá grau 1, ou seja, tal supremo será da forma $a x+b \operatorname{com} a, b \in \mathbb{Z}$ e $a>0$, o que é absurdo pois $a x+b-1$ é uma cota superior de $\mathbb{Z}$ menor que $a x+b$. Portanto, $\mathbb{Z}[x]$ não satisfaz à Propriedade do Supremo.

Exemplo 1.54. O corpo ordenado não arquimediano $\mathcal{R}(x)$ (Exemplo 1.15) não satisfaz à Propriedade do Supremo. 
Exemplo 1.55. O corpo ordenado não arquimediano $\mathcal{L}$ (Exemplo 1.7) não satisfaz à Propriedade do Supremo.

\subsection{Dedekind-Completude}

O corpo ordenado dos números racionais, $\mathbb{Q}$, pode ser particionado da seguinte forma. Sejam

$$
A:=\{x \in \mathbb{Q}: x<2\}
$$

e

$$
B:=\{x \in Q \vdots 2 \leqslant x\} .
$$

Como $A$ e $B$ são conjuntos não vazios disjuntos que cobrem $\mathbb{Q}$, diz-se que eles formam uma partição de $\mathbb{Q}$. Além disso, tal partição preserva a ordem usual em $\mathbb{Q}$, no sentido que todo elemento de $A$ é menor que todo elemento de $B$. Intuitivamente, podemos descrever tal partição como uma divisão geométrica de $\mathbb{Q}$ em duas metades, onde o número racional 2 está exatamente no meio ${ }^{11}$ dessa divisão. Denotaremos tal partição pelo par ordenado $(A, B)$.

É fácil encontrar outros exemplos de partições de $\mathbb{Q}$ em duas metades. Sejam

$$
A^{\prime}:=\left\{x \in \mathbb{Q} \vdots x \leqslant 0 \text { ou } x^{2}<2\right\}
$$

$\mathrm{e}$

$$
B^{\prime}:=\left\{x \in \mathbb{Q} \vdots 0<x \text { e } 2<x^{2}\right\}
$$

Claramente, ${ }^{12} A^{\prime}$ e $B^{\prime}$ são disjuntos. Como o número 2 não tem raiz quadrada racional, o quadrado de qualquer número racional $x$ é distinto de 2 , e verifica-se prontamente que $A^{\prime} \cup B^{\prime}=\mathbb{Q}$. Se $x \in A^{\prime}, y \in B^{\prime}$, e $y<x$, então teremos dois casos:

- Se $y \leqslant 0$, então $y \in A^{\prime}$, o que é absurdo;

- Se $0<y$, então $0<x$ e $2<y^{2}<x^{2}$, resultando em $2<x^{2}$ e $x \in B^{\prime}$, o que é absurdo.

Assim, a partição $\left(A^{\prime}, B^{\prime}\right)$ preserva a ordem usual de $\mathbb{Q}$. Neste caso, porém, não há um número racional exatamente no ponto médio da divisão obtida pela partição considerada.

11 Tecnicamente, temos $2 \in B$, mas estamos ignorando esse fato por enquanto.

12 Basta aplicar (Lin) e as leis de De Morgan

$$
\neg(p \vee q) \Leftrightarrow(\neg p) \wedge(\neg q) \mathrm{e} \neg(p \wedge q) \Leftrightarrow(\neg p) \vee(\neg q),
$$

onde $p$ e $q$ são condições, $\neg$ é a negação de uma condição, $\vee$ é a disjunção de condições, $\wedge$ é a conjunção de condições, e $\Leftrightarrow$ representa a equivalência lógica entre condições. 
Ao que parece, a partição $\left(A^{\prime}, B^{\prime}\right)$ evidencia um "vácuo" existente no corpo ordenado dos números racionais, e presumivelmente existem infinitos outros espaços vazios desse tipo.

A definição a seguir alicerça os conceitos que desenvolvemos nesses dois exemplos.

Definição 1.56. Seja $D$ um domínio ordenado. Um par ordenado $(X, Y)$ é dito ser um corte de Dedekind ${ }^{13}$ em $D$ se:

(Part) $X$ e $Y$ particionam $D$, ou seja, são subconjuntos não nulos de $D$ tais que $X \cap Y=\emptyset$ e $X \cup Y=D$;

(Ord) Para quaisquer $x \in X$ e $y \in Y$, temos $x<y$;

(Mel) $X$ não tem um maior elemento.

Nesse caso, dizemos que o par $(X, Y)$ é uma lacuna em $D$ se $Y$ não tem um menor elemento. Dizemos que $D$ é Dedekind-completo se não existem lacunas em $D$.

Acontece que a Propriedade do Supremo e a ausência de lacunas são dois aspectos de uma mesma propriedade dos domínios ordenados.

Teorema 1.57. O domínio ordenado D será Dedekind-completo se, e somente se, ele observar a Propriedade do Supremo.

Demonstração. Se $D$ obedecer à Propriedade do Supremo, e se $(X, Y)$ for um corte de Dedekind em $D$, então $X$ será um subconjunto não nulo de $D$ que é limitado superiormente, resultando que $X$ terá um supremo $s$ em $D$. Nesse caso, como $X$ não tem um maior elemento, teremos $s \in Y$, e, como todo elemento de $Y$ é uma cota superior de $X$, teremos que $s$ é o menor elemento de $Y$, implicando que $D$ será Dedekind-completo.

Reciprocamente, suponha que $D$ é Dedekind-completo, suponha que $S$ é um subconjunto não nulo de $D$ que não tem um maior elemento e é limitado superiormente, e seja $X$ o conjunto

$$
X:=\{x \in D \vdots(\exists s \in S) x \leqslant s\} .
$$

Nota-se que $X$ não tem um maior elemento, $X$ é limitado superiormente, e o par ordenado $(X, D-X)$ é um corte de Dedekind em $D$. Como esse corte não é uma lacuna em $D$, temos que a diferença $D-X$ tem um menor elemento $s$, o qual claramente é o supremo de $S$ em $D$, provando que $D$ satisfaz à Propriedade do Supremo.

13 O matemático Richard Dedekind (1831-1916) desenvolveu o conceito dos cortes nos números racionais objetivando definir o corpo ordenado dos números reais, $\mathbb{R}$, a partir do corpo ordenado dos números racionais, $\mathbb{Q}$. Por essa razão, tais cortes hoje levam o seu nome. 
Proposição 1.58. Se D for um subdominio ordenado próprio de um domínio ordenado $E$, e se $D$ for denso em $E$, então $D$ não será Dedekind-completo.

Demonstração. Seja $x \in E-D$, e sejam $X$ e $Y$ os conjuntos

$$
\begin{aligned}
& X:=\{y \in D \vdots y<x\} ; \\
& Y:=\{y \in D \vdots x<y\} .
\end{aligned}
$$

Claramente, o par $(X, Y)$ satisfaz aos axiomas (Part) e (Ord), e a densidade de $D$ em $E$ implica diretamente que $X$ não tem um maior elemento e $Y$ não tem um menor elemento.

Exemplo 1.59. O domínio ordenado dos números inteiros, $\mathbb{Z}$, é Dedekind-completo por vacuidade, pois não existem cortes de Dedekind em $\mathbb{Z}$.

Exemplo 1.60. O corpo ordenado dos números racionais, $\mathbb{Q}$, não é Dedekind-completo, visto que $\mathbb{Q}$ é denso em $\mathbb{R}$.

Exemplo 1.61. O corpo ordenado dos números reais, $\mathbb{R}$, é Dedekind-completo. A demonstração desse fato é detalhada em (14). Assim como no caso da Propriedade do Supremo, verifica-se que $\mathbb{R}$ é o único corpo ordenado que é Dedekind-completo, salvo isomorfismos.

Exemplo 1.62. Se $n$ for um número natural que não é um quadrado perfeito, então o domínio ordenado $\mathbb{Z}(\sqrt{n})$ (Exemplo 1.5$)$ não será Dedekind-completo, visto que $\mathbb{Z}(\sqrt{n})$ é denso em $\mathbb{R}$ (Proposição 1.58).

Exemplo 1.63. Os domínios ordenados não arquimedianos $\mathbb{Z}[x], \mathbb{Q}[x]$ e $\mathbb{R}[x]$ (Exemplo 1.6) não são Dedekind-completos.

Exemplo 1.64. O corpo ordenado não arquimediano das funções racionais, $\mathcal{R}(x)$ (Exemplo 1.15), não é Dedekind-completo.

Exemplo 1.65. O corpo ordenado não arquimediano das séries de Laurent, $\mathcal{L}$ (Exemplo 1.7), não é Dedekind-completo. 


\subsection{Cauchy-Completude}

Considere a sequência $\left\{q_{n}\right\}$ de números racionais definida por

- $q_{1}:=1$;

- $q_{n+1}=q_{n}+10^{-n^{2}}(\forall n \geqslant 1)$.

Os primeiros termos dessa sequência são

$$
1 ; 1,1 ; 1,1001 ; 1,100100001 ; 1,1001000010000001 ; \ldots
$$

Percebe-se que os termos da sequência $\left\{q_{n}\right\}$ parecem tender a um número com parte inteira 1, com casas decimais cuja posição é um quadrado perfeito iguais a 1, e com as demais casas decimais iguais a 0 . Tal número não pode ser racional pois suas casas decimais não repetem a mesma sequência finita de dígitos incessantemente.

Prova-se por indução que o $n$-ésimo termo dessa sequência é dado por

$$
q_{n}=\sum_{i=0}^{n} 10^{-i^{2}}(\forall n \geqslant 0) .
$$

Constataremos que os termos da sequência $\left\{q_{n}\right\}$ ficam arbitrariamente próximos uns dos outros a partir de algum índice $n$. Seja $h$ um número racional positivo arbitrariamente pequeno, seja $n_{h}$ um número natural tal que ${ }^{14} 10^{-n_{h}^{2}}<9 h$, e sejam $m$ e $n$ dois números naturais tais que $n_{h}<n<m$. Mostraremos que $\left|q_{m}-q_{n}\right|<h$. Para cada $k=1,2, \ldots, m-n$, temos $k \leqslant k^{2}, 0<2 n k, \mathrm{e}$

$$
n_{h}^{2}+k<n^{2}+k<n^{2}+2 n k+k^{2}=(n+k)^{2}
$$

resultando em

$$
10^{-(n+k)^{2}}<10^{-\left(n_{h}^{2}+k\right)}=10^{-n_{h}^{2}} \cdot 10^{-k}<9 h \cdot 10^{-k} .
$$

Assim, temos

$$
\begin{aligned}
\left|q_{m}-q_{n}\right| & =\left|\sum_{i=0}^{m} 10^{-i^{2}}-\sum_{i=0}^{n} 10^{-i^{2}}\right| \\
& =10^{-(n+1)^{2}}+10^{-(n+2)^{2}}+\cdots+10^{-(n+(m-n))^{2}} \\
& <9 h \cdot 10^{-1}+9 h \cdot 10^{-2}+\cdots+9 h \cdot 10^{-(m-n)} \\
& <9 h \cdot \frac{10^{-1}}{1-10^{-1}} \\
& =h .
\end{aligned}
$$

14 Isso ocorre para algum número natural $n_{h}$ em razão de $\left\{10^{-n^{2}}\right\}$ ser uma sequência decrescente no corpo ordenado $\mathbb{R}$, o qual satisfaz à Propriedade do Supremo. Prova-se que o ínfimo dessa sequência não pode ser positivo e é o limite daquela sequência. 
Embora a sequência $\left\{q_{n}\right\}$ seja constituída inteiramente de números racionais e se aproxime de um determinado valor, ela não tende a um número racional quando $n \rightarrow \infty$. Tal fato indica que os números racionais não são "completos" em um sentido peculiar relacionado a uma classe de sequências que têm "potencial de convergência" mas convergem apenas em uma extensão de $\mathbb{Q}$.

Definição 1.66. Seja $D$ um domínio ordenado, e seja

$$
\left\{x_{n}\right\}=x_{1} x_{2} x_{3} \ldots
$$

uma sequência em $D$.

- Para $l \in D$, dizemos que $l$ é um limite de $\left\{x_{n}\right\}$ em $D$ se para todo $h>0$ em $D$ existe um número natural $n_{h}$ tal que para todo número natural $n$ temos

$$
n_{h} \leqslant n \Rightarrow\left|x_{n}-l\right|<h
$$

Nesse caso, dizemos que a sequência $\left\{x_{n}\right\}$ é convergente em $D$, e denotamos o elemento $l \in D$ por $\lim x_{n}$;

- Dizemos $\left\{x_{n}\right\}$ é uma sequência de Cauchy em $D$ se para todo $h>0$ em $D$ existe um número natural $n_{h}$ tal que para quaisquer números naturais $m$ e $n$ temos

$$
m, n \geqslant n_{h} \Rightarrow\left|x_{m}-x_{n}\right|<h
$$

- Dizemos que $D$ é Cauchy-completo se toda sequência de Cauchy em $D$ é convergente em $D$.

Se $l$ e $l^{\prime}$ forem limites da sequência $\left\{x_{n}\right\}$ em $D$, então teremos $l=l^{\prime}$. Isso ocorre pois se $h$ for um elemento positivo em $D$, então existirão números naturais $n_{h / 2}$ e $n_{h / 2}^{\prime}$ tais que $\left|x_{n}-l\right|<h / 2$ e $\left|x_{n^{\prime}}-l^{\prime}\right|<h / 2$ para quaisquer $n \geqslant n_{h / 2}$ e $n^{\prime} \geqslant n_{h / 2}^{\prime}$. Nesse caso, se $n \geqslant \max \left\{n_{h / 2}, n_{h / 2}^{\prime}\right\}$, então teremos

$$
\begin{aligned}
\left|l-l^{\prime}\right| & =\left|\left(l-x_{n}\right)+\left(x_{n}-l^{\prime}\right)\right| \\
& \leqslant\left|l-x_{n}\right|+\left|x_{n}-l^{\prime}\right| \\
& <\frac{h}{2}+\frac{h}{2} \\
& =h,
\end{aligned}
$$

e, como $h$ é arbitrário, teremos $l=l^{\prime}$.

Proposição 1.67. Se D for um subdominio ordenado próprio de um domínio ordenado arquimediano $E$, se $D$ for denso em $E$, e se todo número natural for inversivel em $E$, então D não será Cauchy-completo. 
Demonstração. Seja $l \in E-D$. Como o domínio ordenado $D$ é denso em $E$, para cada número natural $n$ existe um $x_{n} \in D$ tal que $\left|x_{n}-l\right|<1 / 2 n$. Como $E$ é arquimediano, temos $x_{n} \rightarrow l$ quando $n \rightarrow \infty$, visto que todo elemento positivo de $E$ é maior que algum número racional da forma $1 / 2 n$ para algum número natural $n$ suficientemente grande. Se $h$ for um elemento positivo de $D$, então existirá um número natural $n_{h}$ com $1 / n_{h}<h$, e se além disso $m$ e $n$ forem números naturais maiores que $n_{h}$, então teremos

$$
\begin{aligned}
\left|x_{m}-x_{n}\right| & \leqslant\left|x_{m}-l\right|+\left|l-x_{n}\right| \\
& <\frac{1}{2 m}+\frac{1}{2 n} \\
& <\frac{1}{2 n_{h}}+\frac{1}{2 n_{h}} \\
& =\frac{1}{n_{h}} \\
& <h
\end{aligned}
$$

provando que $\left\{x_{n}\right\}$ é uma sequência de Cauchy.

Exemplo 1.68. O domínio ordenado dos números inteiros, $\mathbb{Z}$, é Cauchy-completo. De fato, toda sequência de Cauchy em $\mathbb{Z}$ é eventualmente constante.

Exemplo 1.69. Como vimos, $\left\{q_{n}\right\}$ é uma sequência de Cauchy que não é convergente em $\mathbb{Q}$. Portanto, o corpo ordenado $\mathbb{Q}$ não é Cauchy-completo, e, como $\mathbb{Q}$ é um domínio ordenado imerso em $\mathbb{Q}[x]$, temos que $\mathbb{Q}[x]$ não é Cauchy-completo.

Exemplo 1.70. O corpo ordenado dos números reais, $\mathbb{R}$, é Cauchy-completo. Tal fato é provado em (14).

Exemplo 1.71. Se $n$ for um número natural que não é um quadrado perfeito, então o domínio ordenado $\mathbb{Z}(\sqrt{n})$ (Exemplo 1.5$)$ não será Cauchy-completo, visto que $\mathbb{Z}(\sqrt{n})$ é denso em $\mathbb{R}$ e que todo número natural é inversível em $\mathbb{R}$ (Proposição 1.67).

Exemplo 1.72. Seja $\left\{\sum_{i=0}^{q_{n}} c_{n, i} x^{i}\right\}$ uma sequência arbitrária em $\mathbb{R}[x]$ (Exemplo 1.6). Suponha que essa sequência é de Cauchy. Assim, para todo $h>0$ em $\mathbb{R}[x]$ existe um número natural $n_{h}$ tal que para quaisquer números naturais $m$ e $n$ temos

$$
m, n \geqslant n_{h} \Rightarrow\left|\sum_{i=0}^{q_{m}} c_{m, i} x^{i}-\sum_{i=0}^{q_{n}} c_{n, i} x^{i}\right|<h
$$

Em particular, se $h$ for um número real positivo e $m, n \geqslant n_{h}$, então os polinômios $\sum_{i=0}^{q_{m}} c_{m, i} x^{i}$ e $\sum_{i=0}^{q_{n}} c_{n, i} x^{i}$ terão o mesmo grau e diferirão apenas no coeficiente da potência $x^{0}$, ou seja, teremos $q_{m}=q_{n}, c_{m, i}=c_{n, i}\left(\forall i \in\left[1, q_{m}\right]_{\mathbb{N}_{0}}\right)$, e $\left|c_{m, 0}-c_{n, 0}\right|<h$. Seja $q$ o valor constante 
que a sequência $\left\{q_{n}\right\}_{n \in \mathbb{N}}$ eventualmente assume, e, para cada número natural $i$ com $1 \leqslant i \leqslant q$, seja $c_{i}$ o valor constante que a sequência $\left\{c_{n, i}\right\}_{n \in \mathbb{N}}$ eventualmente assume. A sequência $\left\{c_{n, 0}\right\}_{n \in \mathbb{N}}$ é de Cauchy em $\mathbb{R}$, e, como $\mathbb{R}$ é Cauchy-completo, tal sequência converge para um número real $c_{0}$. Prova-se diretamente que

$$
\left\{\sum_{i=0}^{q_{n}} c_{n, i} x^{i}\right\} \rightarrow\left\{\sum_{i=0}^{q} c_{i} x^{i}\right\}
$$

quando $n \rightarrow \infty$. Portanto, $\mathbb{R}[x]$ é Cauchy-completo. Uma demonstração semelhante mostra que $\mathbb{Z}[x]$ é Cauchy-completo.

Exemplo 1.73. Considere a sequência $\left\{a_{n}(x)\right\}$ em $\mathcal{R}(x)$ (Exemplo 1.15) dada por

$$
a_{n}(x):=\frac{1}{x^{n}} \cdot \sum_{i=0}^{n-1} x^{i}=\sum_{i=1}^{n} \frac{1}{x^{i}}
$$

para $n \geqslant 1$, a qual tem seus primeiros termos iguais a

$$
\frac{1}{x}, \frac{x+1}{x^{2}}, \frac{x^{2}+x+1}{x^{3}}, \frac{x^{3}+x^{2}+x+1}{x^{4}}, \frac{x^{4}+x^{3}+x^{2}+x+1}{x^{5}}, \ldots
$$

Seja $u(x) / v(x)>0$ em $\mathcal{R}(x), \operatorname{com} u(x)=\sum_{i=0}^{k} g_{i} x^{i}, g_{k} \neq 0, v(x)=\sum_{i=0}^{l} h_{i} x^{i}$, e $h_{l}=1$. Se $m$ e $n$ forem números naturais tais que $l-k-1<n<m$, então

$$
(m-n-1)+l<m+k,
$$

resultando em

$$
\begin{aligned}
\left|a_{m}(x)-a_{n}(x)\right| & =\sum_{i=1}^{m} \frac{1}{x^{i}}-\sum_{i=1}^{n} \frac{1}{x^{i}} \\
& =\sum_{i=n+1}^{m} \frac{1}{x^{i}} \\
& =\frac{1}{x^{n}} \cdot \sum_{i=1}^{m-n} \frac{1}{x^{i}} \\
& =\frac{1}{x^{n}} \cdot \frac{1}{x^{m-n}} \cdot \sum_{i=0}^{m-n-1} x^{i} \\
& =\frac{1+x+x^{2}+\cdots+x^{m-n-1}}{x^{m}} \\
& <\frac{u(x)}{v(x)},
\end{aligned}
$$

e provando que $\left\{a_{n}(x)\right\}$ é uma sequência de Cauchy.

Provaremos que a sequência $\left\{a_{n}(x)\right\}$ não é convergente. Suponha que $p(x) / q(x)$ é o limite dessa sequência em $\mathcal{R}(x), \operatorname{com} p(x)=\sum_{i=1}^{r} c_{i} x^{i}, c_{r} \neq 0, q(x)=\sum_{i=1}^{s} d_{i} x^{i}$, e 
$d_{s}=1$. Como $\left\{a_{n}(x)\right\}$ é crescente, a fração $p(x) / q(x)$ é o supremo dessa sequência, e temos $1 / x<p(x) / q(x)$, resultando em $0<x \cdot p(x)-q(x)$ e $s \leqslant r+1$. O cenário $s \leqslant r$ pode ser facilmente descartado visto que nesse caso teríamos $c_{r} / 2<p(x) / q(x)$, contradizendo o fato de que $p(x) / q(x)$ é o supremo da sequência $\left\{a_{n}(x)\right\}$. Portanto, temos $s=r+1$, e, como $0<x \cdot p(x)-q(x)$, temos $1<c_{r}$. Nota-se que $a_{1}(x)<(x+2) / x^{2}<p(x) / q(x)$, e temos

$$
\begin{aligned}
a_{n}(x) & =\frac{1}{x}+\frac{1}{x^{2}}+\frac{1}{x^{3}}+\cdots+\frac{1}{x^{n}} \\
& <\frac{1}{x}+\frac{1}{x^{2}}+\overbrace{\frac{1}{(n-2) x^{2}}+\frac{1}{(n-2) x^{2}}+\cdots+\frac{1}{(n-2) x^{2}}}^{n-2 \text { vezes }} \\
& =\frac{x+2}{x^{2}}
\end{aligned}
$$

para todo $n>1$, ou seja, $(x+2) / x^{2}$ é uma cota superior da sequência $\left\{a_{n}(x)\right\}$, o que é absurdo pois $p(x) / q(x)$ é o supremo dessa sequência. Provamos que $\left\{a_{n}(x)\right\}$ não é convergente, e o corpo ordenado $\mathcal{R}(x)$ não é Cauchy-completo.

Exemplo 1.74. Seja $\left\{\sum_{i=p_{n}}^{\infty} c_{n, i} x^{i}\right\}$ uma sequência arbitrária em $\mathcal{L}$ (Exemplo 1.7). Suponha que essa sequência é de Cauchy. Isso significa que para todo $h(x)>0$ em $\mathcal{L}$ existe um número natural $n_{h}$ tal que

$$
\left|\sum_{i=p_{u}}^{\infty} c_{u, i} x^{i}-\sum_{i=p_{v}}^{\infty} c_{v, i} x^{i}\right|<h(x)
$$

para quaisquer $u$ e $v$ maiores ou iguais a $n_{h}$. Em particular, para cada número inteiro $k$ existe um número natural $n_{k}$ tal que

$$
\left|\sum_{i=p_{u}}^{\infty} c_{u, i} x^{i}-\sum_{i=p_{v}}^{\infty} c_{v, i} x^{i}\right|<x^{k}
$$

para quaisquer números naturais $u$ e $v$ maiores ou iguais a $n_{k}$.

Se $i<\min \left\{p_{n_{0}}, 0\right\}$, então $c_{n_{0}, i}=0$, e, como

$$
\left|\sum_{i=p_{u}}^{\infty} c_{u, i} x^{i}-\sum_{i=p_{n_{0}}}^{\infty} c_{n_{0}, i} x^{i}\right|<x^{0}=1\left(\forall u \geqslant n_{0}\right)
$$

temos $c_{u, i}=0\left(\forall u \geqslant n_{0}\right)$. Definindo $p:=\min \left\{p_{n_{0}}, 0\right\}$, temos

$$
c_{u, i}=0\left(\forall u \geqslant n_{0}\right)(\forall i<p)
$$

Em particular, para cada número inteiro $i<p$ a sequência $\left\{c_{n, i}\right\}_{n \geqslant 1}$ eventualmente estabiliza-se no valor 0 . 
Seja $k$ um número inteiro qualquer, e sejam $u$ e $v$ dois números naturais maiores ou iguais a $n_{k}$. Por indução sobre $i$, prova-se diretamente que $c_{u, i}-c_{v, i}=0(\forall i<k)$. Como $k$ é arbitrário, temos que para cada número inteiro $i$ a sequência $\left\{c_{n, i}\right\}_{n \geqslant 1}$ eventualmente estabiliza-se em um número real $r_{i}$ para todo $n$ maior ou igual a um número natural $N_{i}$, ou seja, $c_{n, i}=r_{i}\left(\forall n \geqslant N_{i}\right)$. Pelo parágrafo anterior, temos $r_{n}=0(\forall n<p)$ e $\left\{r_{n}\right\} \in \mathcal{L}$.

$\mathcal{L} \operatorname{com} 0<e_{a}$, e seja

Mostraremos que $\sum_{i=p_{n}}^{\infty} c_{n, i} x^{i} \rightarrow \sum_{i=p}^{\infty} r_{i} x^{i}$ quando $n \rightarrow \infty$. Seja $h(x):=\sum_{i=a}^{\infty} e_{i} x^{i}$ em

$$
n_{h}:=\max \left\{n_{0}, N_{\min \{p, a\}}, N_{\min \{p, a\}+1}, \ldots, N_{\max \{p, a\}}\right\} .
$$

Assim, se $n$ for um número natural com $n_{h} \leqslant n$, então $c_{n, i}=r_{i}(\forall i \leqslant \max \{p, a\})$, e teremos

$$
\left|\sum_{i=p_{n}}^{\infty} c_{n, i} x^{i}-\sum_{i=p}^{\infty} r_{i} x^{i}\right|=\left|\sum_{i=\max \{p, a\}+1}^{\infty}\left(c_{n, i}-r_{i}\right) \cdot x^{i}\right|<h(x),
$$

provando que a sequência $\left\{\sum_{i=p_{n}}^{\infty} c_{n, i} x^{i}\right\}$ é convergente e o corpo ordenado $\mathcal{L}$ é Cauchy-completo.

Na Seção 5.6 veremos um exemplo de um corpo ordenado tal que toda sequência de Cauchy é eventualmente constante, e, por conseguinte, tal corpo ordenado é Cauchy-completo.

O teorema a seguir tem importância substancial para a teoria dos corpos ordenados, e sua demonstração pode ser encontrada em (14). Além de estabelecer um vínculo entre os principais conceitos vistos neste capítulo, ele revela a singularidade do corpo ordenado dos números reais.

Teorema 1.75. Seja K um corpo ordenado. As seguintes condições são equivalentes:

- K é arquimediano e Cauchy-completo;

- K satisfaz à Propriedade do Supremo;

- $K$ é Dedekind-completo.

De fato, o corpo ordenado dos números reais, $\mathbb{R}$, é o único corpo ordenado que satisfaz às condições equivalentes mencionadas, salvo isomorfismos.

Sintetizando o que vimos até este ponto, podemos agrupar os domínios e corpos ordenados em quatro classes: 
Tabela 2 - Classificação dos domínios ordenados

\begin{tabular}{c|c|c|}
\multicolumn{1}{c}{} & Cauchy-incompleto & Cauchy-completo \\
\cline { 2 - 3 } Não arquimediano & $\mathcal{R}(x), \mathbb{Q}[x]$ & $\mathbb{Z}[x], \mathbb{R}[x], \mathcal{L}$ \\
\hline Arquimediano & $\mathbb{Q}, \mathbb{Z}(\sqrt{n})$ & $\mathbb{Z}, \mathbb{R}$ \\
\cline { 2 - 3 } & &
\end{tabular}

Tabela 3 - Classificação dos corpos ordenados

\begin{tabular}{|c|c|c|}
\hline & Cauchy-incompleto & Cauchy-completo \\
\hline Não arquimediano & $\mathcal{R}(x)$ & $\mathcal{L}$ \\
\hline Arquimediano & $\mathbb{Q}$ & $\mathbb{R}$ apenas, salvo isomorfismos \\
\hline
\end{tabular}

\subsection{Domínios Ordenados que Estendem $\mathbb{R}$}

Nesta seção, considere que existe uma imersão $\mathbb{R} \rightarrow D$ (Definição A.42) entre domínios ordenados. Assim, podemos identificar os elementos da imagem dessa imersão com os números reais, e, por abuso de linguagem, podemos escrever $\mathbb{R} \subset D$. Todo número real é finito em $D$.

Exemplo 1.76. O domínio ordenado $\mathbb{R}[x]$ (Exemplo 1.6) é uma extensão de $\mathbb{R}$.

Exemplo 1.77. O corpo ordenado das funções racionais, $\mathcal{R}(x)$ (Exemplo 1.15), é uma extensão de $\mathbb{R}$.

Exemplo 1.78. O corpo ordenado das séries de Laurent, $\mathcal{L}$ (Exemplo 1.7), é uma extensão de $\mathbb{R}$.

Proposição 1.79. O número zero é o único infinitesimal real em D.

Demonstração. Seja $r$ um infinitesimal real em $D$. Se $r \neq 0$, então $|r| \neq 0$, e, como $\mathbb{R}$ é um corpo ordenado arquimediano, existirá um número natural $n$ tal que $1 \leqslant n|r|$, o que é absurdo pois $r$ é infinitesimal.

Teorema 1.80. Se $x$ for um elemento finito de D, então existirá um único número real $r$ tal que $x \sim r$.

Demonstração. Como $x$ é finito, o conjunto

$$
A:=\{y \in \mathbb{R}: y<x\}
$$

é limitado superiormente em $\mathbb{R}$. Seja $r:=\sup _{\mathbb{R}}(A)$. Provaremos que $x \sim r$, e para isso basta mostrar que $|r-x| \leqslant h$ para todo número real positivo $h$. Seja $h$ um número desse tipo. Se $r<x-h$, então $r+h<x, r+h \in A$, e $r+h \leqslant r$, o que é absurdo pois $0<h$. 
Se $x+h<r$, então $x<r-h$ e $r-h$ é uma cota superior de $A$ em $\mathbb{R}$, o que é absurdo pois $0<h$ e $r-h<r=\sup _{\mathbb{R}}(A)$. Portanto, temos

$$
x-h \leqslant r \leqslant x+h,
$$

ou seja, $|r-x|<h$, mostrando que $x \sim r$. A unicidade de $r$ é uma consequência imediata da Proposição 1.79 e da transitividade da relação de equivalência $\sim$.

Todo elemento finito $x$ de $D$ pode ser escrito na forma st $(x)+e$, onde $e$ é um infinitesimal unicamente determinado por $x$.

Definição 1.81. A função parte standard associada a $D, \operatorname{st}_{D}:$ Fin $(D) \rightarrow \mathbb{R}$, é a função que associa cada elemento finito $x$ em $D$ ao único número real $\operatorname{st}_{D}(x)$ tal que $x \sim \operatorname{st}_{D}(x)$. Denotaremos tal função simplesmente por st quando o domínio ordenado $D$ em questão estiver implícito no contexto.

$\operatorname{Temos} \operatorname{Im}(\mathrm{st})=\mathbb{R}$ e $\operatorname{ker}(\mathrm{st})=\operatorname{Inf}(D)$

Proposição 1.82. A função st $:$ Fin $(D) \rightarrow \mathbb{R}$ é um morfismo entre domínios não estritamente ordenados. Ou seja, as seguintes condições são observadas para quaisquer $x, y \in \operatorname{Fin}(D)$ :
(a) $\operatorname{st}(1)=1$;
(c) $\quad$ st $(x \cdot y)=\operatorname{st}(x) \cdot \operatorname{st}(y)$;
(b) $\quad$ st $(x+y)=\operatorname{st}(x)+$ st $(y)$;
(d) Se $x \leqslant y$, então st $(x) \leqslant \operatorname{st}(y)$.

Demonstração. As letras $(a)$, (b) e (c) são consequências imediatas do Teorema 1.18.

(d) Provaremos primeiro que para $z$ finito em $D$ vale a implicação $0 \leqslant z \Rightarrow 0 \leqslant \operatorname{st~}(z)$. Seja $z \in \operatorname{Fin}(D)$, e seja $e$ o infinitesimal em $D$ tal que $z=\operatorname{st}(z)+e$. Se $\operatorname{st}(z)<0$, então $|\operatorname{st}(z)|$ não será infinitesimal (Proposição 1.79) e teremos

$$
|e|<|\operatorname{st}(z)|=-\operatorname{st}(z)
$$

ou seja, st $(z)<e<-$ st $(z)$, resultando em $0<-$ st $(z)-e=-z$ e $z<0$, e provando a implicação desejada. Se $x \leqslant y, \operatorname{com} x$ e $y$ finitos, então $0 \leqslant y-x$ e

$$
0 \leqslant \operatorname{st}(y-x)=\operatorname{st}(y)-\operatorname{st}(x) .
$$

O Primeiro Teorema do Isomorfismo (Teorema 1.40) implica que o domínio ordenado quociente Fin $(D) / \operatorname{Inf}(D)$ é isomorfo a $\mathbb{R}$. 


\subsection{Análise Diferencial e Integral em Extensões de $\mathbb{R}$}

A Análise Real é repleta de resultados amplamente aplicáveis a diversas áreas do conhecimento, como a Física e as Engenharias. A ampla aplicabilidade dos números reais decorre em razão de eles modelarem satisfatoriamente a intuição que temos das grandezas contínuas como as distâncias e o tempo. De fato, é possível argumentar que nenhum outro objeto matemático foi tão útil e benéfico ao desenvolvimento teórico-científico como o conjunto $\mathbb{R}$.

Porém, tal corpo ordenado é arquimediano. Isso significa que ele não contém quantidades infinitesimais e infinitas, as quais são bastante úteis na exploração de novos conceitos e resultados na Ciência e na Matemática. Essa aparente imperfeição levou muitos a questionarem a possibilidade de substituir o conjunto dos números reais por uma extensão deste a um corpo ordenado não arquimediano que ainda goze de muitas das qualidades que os números reais possuem.

É desejável que o desenvolvimento de uma teoria acerca de um substituto de $\mathbb{R}$ contenha resultados precisos, notáveis e utilitários sobre a convergência de séries de potências, as expansões de funções diferenciáveis em séries de Taylor e as equações diferenciáis ordinárias e parciais, entre outros temas. Ademais, versões renovadas de teoremas intuitivos relacionados à noção de continuidade, como o Teorema do Valor Intermediário, o Teorema do Valor Médio e o Teorema Fundamental do Cálculo, são indispensáveis para a teoria de um sistema numérico que seja proposto para sobrepor os números reais. Duas construções não arquimedianas apresentadas que cumprem essas demandas são os corpos de números surreais $(16,24,31)$ e o corpo de Levi-Civita $(48,49)$. Construído mediante uma variedade de métodos da área conhecida como Análise Não Standard, o corpo ordenado dos números hiper-reais, o qual é denotado por * $\mathbb{R}$, é um sistema numérico não arquimediano que satisfaz a todas as propriedades desejáveis para uma extensão de $\mathbb{R}$. A edificação da Análise Hiper-real como uma proposta persuasiva de substituição de $\mathbb{R}$ por ${ }^{*} \mathbb{R}$ está fora do escorpo deste trabalho, e pode ser encontrada em $(43,45,50)$.

No Capítulo 2, estabeleceremos de modo informal os princípios que regem o comportamento e a aplicabilidade dos números hiper-reais. Veremos que o resultado conhecido como Princípio da Transferência permite que determinações sejam bilateralmente transferidas entre os sistemas dos números reais e dos números hiper-reais. Essa peculiaridade viabiliza um procedimento proveitoso que permite que vários resultados da Análise Real sejam provados de maneira mais espontânea, direta e intuitiva. 


\section{2 \\ Visão Ingênua dos Números Hiper-reais}

\subsection{Os Números Hiper-reais}

Neste capítulo trataremos informalmente de um corpo ordenado não arquimediano denotado por ${ }^{*} \mathbb{R}$ cujos elementos são ditos serem números hiper-reais. A construção formal dessa estrutura será mostrada apenas nos Capítulos 3 e 5, e aqui abordaremos apenas as propriedades de ${ }^{*} \mathbb{R}$ que o tornam úteis para o estudo da Análise Real.

O que faz ${ }^{*} \mathbb{R}$ ser especial e incomum em relação a qualquer outra extensão não arquimediana de $\mathbb{R}$, como $\mathcal{R}(x)$ e $\mathcal{L}$ ? Como veremos, há um vínculo essencial entre os números reais e hiper-reais, o qual permite que vários resultados fundamentais do Cálculo Diferencial e Integral em $\mathbb{R}$ sejam provados de maneira direta e intuitiva, aproximando-se do caráter das demonstrações originais de Leibniz e Euler nos séculos XVII e XVIII.

Exploraremos os princípios que regem a conexão entre $\mathbb{R}$ e ${ }^{*} \mathbb{R}$, e enunciaremos novas interpretações para as noções básicas do Cálculo.

\subsection{Objetos Relacionados a $\mathbb{R} e^{*} \mathbb{R}$}

Quais objetos matemáticos são potencialmente relevantes ao estudo da Análise Real? Os números reais e o conjunto $\mathbb{R}$ são certamente relevantes nessa área, e incontáveis exemplos mostram que vários outros objetos e conjuntos podem ser considerados nos resultados da teoria. Intervalos em $\mathbb{R}$ são citados no Teorema de Heine-Borel; uniões de sequências crescentes de conjuntos abertos em $\mathbb{R}^{n}$ são importantes em critérios de integrabilidade; produtos cartesianos finitos são usados para definir funções reais de mais de uma variável; e o conjunto das partes de $\mathbb{N}$ é citado em resultados que envolvem o cardinal de $\mathbb{R}$. É admissível assumir que a coleção dos conjuntos pertinentes na Análise Real é fechado sob algumas operações básicas da Teoria dos Conjuntos. A definição a seguir é uma tentativa de formalizar essa ideia.

Definição 2.1 (Objetos Relacionados a $\mathbb{R}$ e ${ }^{*} \mathbb{R}$ - Versão Ingênua).

(a) Dizemos que os números reais são átomos relacionados a $\mathbb{R}$ ou $\mathbb{R}$-átomos;

(b) Definiremos o conceito dos conjuntos relacionados a $\mathbb{R}$ ou $\mathbb{R}$-conjuntos indutivamente de modo que:

1. $\mathbb{R}$ é um $\mathbb{R}$-conjunto;

2. Todo subconjunto de um $\mathbb{R}$-conjunto é um $\mathbb{R}$-conjunto; 
3. Se $A$ for um $\mathbb{R}$-conjunto, então $\mathcal{P}(A)$ será um $\mathbb{R}$-conjunto;

4. Toda união de $\mathbb{R}$-conjuntos é um $\mathbb{R}$-conjunto.

(c) Dizemos que um objeto matemático é um objeto relacionado a $\mathbb{R}$ ou $\mathbb{R}$-objeto se ele pertence a algum conjunto relacionado a $\mathbb{R}$.

Definimos os conceitos de átomo, conjunto e objeto relacionado $\mathbf{a}{ }^{*} \mathbb{R}$ de modo análogo.

Nos Capítulos 4 e 5, veremos que, para os propósitos desta dissertação, será necessário restringir a propriedade 4 de modo que apenas algumas uniões específicas de $\mathbb{R}$-conjuntos necessariamente serão $\mathbb{R}$-conjuntos (Teorema 4.8, Letra $(d)$ ).

O leitor familiarizado com a Teoria dos Conjuntos perceberá que a classe dos objetos relacionados a $\mathbb{R}\left(\right.$ resp. ${ }^{*} \mathbb{R}$ ) será igual ao universo de von Neumann (Exemplo B.8), o qual é dado por (Seção B.5)

$$
\mathrm{V}=\{x: x=x\}=\bigcup_{\gamma \in \mathrm{On}} \mathrm{V}_{\gamma}
$$

Portanto, a investida para definir os objetos relacionados a $\mathbb{R}$, representada pela Definição 2.1, excede severamente a meta de identificar os objetos matemáticos que são pertinentes à investigação da Análise Real. Esse quesito será ignorado neste capítulo, sendo propriamente ponderado apenas no Capítulo 4.

Será importante para a discussão subsequente que nenhum átomo relacionado a $\mathbb{R}\left(\right.$ resp. $\left.{ }^{*} \mathbb{R}\right)$ tenha um objeto relacionado a $\mathbb{R}\left(\right.$ resp. $\left.{ }^{*} \mathbb{R}\right)$ como elemento. Na Seção 5.1, veremos como obter uma cópia de $\mathbb{R}$ tal que esse requisito seja cumprido.

\section{Proposição 2.2.}

(a) Se a e b forem $\mathbb{R}$-objetos, então $\{a, b\}$ será um $\mathbb{R}$-conjunto;

(b) Se a e b forem $\mathbb{R}$-objetos, então $(a, b)$ será um $\mathbb{R}$-conjunto;

(c) Se $A$ e $B$ forem $\mathbb{R}$-conjuntos, então $A \times B$ será um $\mathbb{R}$-conjunto.

Tais afirmações são verdadeiras ao trocarmos $\mathbb{R}$ por ${ }^{*} \mathbb{R}$.

Demonstração.

(a) Se $A$ e $B$ forem $\mathbb{R}$-conjuntos tais que $a \in A$ e $b \in B$, então $A \cup B$ será um $\mathbb{R}$-conjunto, e, como $\{a, b\} \subset A \cup B$, teremos que $\{a, b\}$ será um $\mathbb{R}$-conjunto. 
A letra $(b)$ é consequência imediata da $(a)$.

(c) Se $a \in A$ e $b \in B$, então, pelas letras $(a)$ e $(b)$, teremos que $(a, b)$ e $\{(a, b)\}$ são $\mathbb{R}$-conjuntos, implicando que a união

$$
A \times B=\bigcup_{a \in A, b \in B}\{(a, b)\}
$$

será um $\mathbb{R}$-conjunto.

As provas dessas afirmações são análogas ao trocarmos $\mathbb{R}$ por * $\mathbb{R}$.

Em geral, assumiremos que o leitor tem a mestria para demonstrar que um objeto matemático é relacionado a $\mathbb{R}$ ou ${ }^{*} \mathbb{R}$, e nos pouparemos desse tipo de incumbência neste trabalho.

\subsection{Condições com Quantificadores Limitados}

Neste capítulo, quando nos referirmos informalmente a uma condição matemática $\phi\left(x_{1} \ldots x_{n}\right)$, estaremos assumindo que ela observa aos seguintes requisitos:

- Toda variável livre que aparece em $\phi$ pertence à lista de variáveis $x_{1} \ldots x_{n}$. As variáveis ligadas em $\phi$ podem ser quaisquer variáveis que não estejam na lista $x_{1} \ldots x_{n}$;

- $\phi$ é completamente descrita por símbolos basilares da Teoria dos Conjuntos, os quais são:

- Objetos matemáticos fixos, como $2, \pi, \mathbb{C}, 1 / 3, \omega_{1}$;

- Os símbolos lógicos: $\neg, \vee, \wedge, \Rightarrow, \Leftrightarrow,=, \neq$;

- Os quantificadores (prefixais): $\exists, \exists !, \forall$;

- Os delimitadores: $),(],,[\},,\{$;

- A relação de pertinência: $\in$;

- A notação de par ordenado: $(x, y)$ representa o par ordenado cuja primeira coordenada é $x$ e cuja segunda coordenada é $y$;

- A notação de aplicação funcional prefixal: $x(y)$ representa a imagem de um objeto $y$ sobre a função $x$;

- A notação de aplicação funcional infixal: $x y z$ representa a imagem do par ordenado cujas respectivas primeira e segunda coordenadas são $x$ e $z$ sobre a lei de composição $y$; 
- A notação de vinculação relacional infixal: $x y z$ representa que o par ordenado cujas respectivas primeira e segunda coordenadas são $x$ e $z$ pertence à relação binária $y$.

- Cada ocorrência do quantificador existencial $\exists x$ (lê-se "existe um $x$ ") e do quantificador universal $\forall x$ (lê-se "para todo $x$ ") em $\phi$ está semanticamente limitada a um universo delimitado, de modo que tais quantificadores aparecem nessa condição apenas nas formas $\forall x \in y$ e $\exists x \in y$, onde $y$ pode ser uma variável (livre ou ligada) ou um objeto matemático fixo.

Se pelo menos uma variável da lista $x_{1} \ldots x_{n}$ aparece livre em $\phi$, então dizemos que $\phi$ é aberta, e caso contrário dizemos que $\phi$ é fechada. Se $\phi$ é aberta e nenhum objeto matemático fixo aparece em $\phi$, então dizemos que $\phi$ é completamente aberta. Condições fechadas ou são verdadeiras ou são falsas. Se todo objeto matemático fixo que aparece em $\phi$ é um objeto relacionado a $\mathbb{R}$ (resp. ${ }^{*} \mathbb{R}$ ), então dizemos que $\phi$ é uma condição relacionada a $\mathbb{R}\left(\right.$ resp. $\left.{ }^{*} \mathbb{R}\right)$ ou uma $\mathbb{R}$-condição (resp. ${ }^{*} \mathbb{R}$-condição).

\section{$2.4 *$ *-Transformações e o Princípio da Transferência}

O teorema a seguir é a principal ferramenta desta dissertação, e ele é utilizado sistematicamente em todo o estudo da Análise Não Standard. Desenvolveremos duas maneiras distintas de prová-lo nos Capítulos 3 e 5, onde o enunciaremos de maneiras mais precisas e gerais.

Teorema 2.3 (*-Transformações de Objetos - Versão Ingênua). Existe uma função injetora $*$ tal que:

(a) O domínio de * é o conjunto dos $\mathbb{R}$-objetos;

(b) O contradomínio de * é o conjunto dos $* \mathbb{R}$-objetos;

(c) $*(x)=x(\forall x \in \mathbb{R})$;

(d) $*(\mathbb{R})=* \mathbb{R}$

(e) Se $A$ for um $\mathbb{R}$-conjunto, então $*\langle A\rangle \subset *(A)$, e se além disso A for infinito, então $*\langle A\rangle \subsetneq *(A)$. Em particular, $\mathbb{N} \subsetneq *(\mathbb{N})$ e $\mathbb{R} \subsetneq *(\mathbb{R})$;

(f) (Princípio da Transferência; PT) Seja $\phi\left(x_{1} \ldots x_{n}\right)$ uma condição completamente aberta, e seja $a_{1} \ldots a_{n}$ uma sequência de $\mathbb{R}$-objetos. A condição fechada $\phi\left(a_{1} \ldots a_{n}\right)$ será verdadeira se, e somente se, a condição fechada $\phi\left(*\left(a_{1}\right) \cdots *\left(a_{n}\right)\right)$ for verdadeira. 
Tal função * é dita ser um monomorfismo não standard.

A letra $(f)$ do Teorema 2.3, conhecida como Princípio da Transferência $(\mathbf{P T})$, estabelece um elo lógico entre as propriedades dos $\mathbb{R}$-objetos e as propriedades dos $* \mathbb{R}$-objetos.

A condição fechada $\phi\left(*\left(a_{1}\right) \cdots *\left(a_{n}\right)\right)$ relacionada a $* \mathbb{R}$, mencionada no enunciado do PT, é dita ser a $*$-transformação da condição fechada $\phi\left(a_{1} \ldots a_{n}\right)$.

Notação 2.4. Se $a$ for um $\mathbb{R}$-objeto, então a $*$-transformação de $a$, dada por $*(a)$, será denotada por ${ }^{*} a$.

Corolário 2.5 (Propriedades de * - Versão Ingênua). Sejam a e b dois $\mathbb{R}$-objetos, e sejam $A$ e $B$ dois $\mathbb{R}$-conjuntos. Temos:
(a) $*\langle A\rangle \subset{ }^{*} A$
(d) $A \subset B \Leftrightarrow{ }^{*} A \subset{ }^{*} B$
(b) $a=b \Leftrightarrow{ }^{*} a={ }^{*} b$;
(c) $\quad a \in A \Leftrightarrow{ }^{*} a \in{ }^{*} A$
(e) ${ }^{*} \emptyset=\emptyset$.

Demonstração. A letra (a) é consequência evidente da letra (e) do Teorema 2.3, e a letra (b) é uma releitura da injetividade da função *. Os resultados das letras $(c),(d)$ e $(e)$ seguem ao aplicarmos o Princípio da Transferência às respectivas condições fechadas dadas por $a \in A,(\forall x \in A)(x \in B)$ e $(\forall x \in \emptyset)(x \neq x)$.

\subsection{Objetos Standard e Não Standard}

A função * não é sobrejetora, ou seja, existem * ${ }^{*}$-objetos que não são *-transformações de algum $\mathbb{R}$-objeto. Considere, a título de exemplo, o conjunto * $A-*\langle A\rangle$, onde $A$ é um $\mathbb{R}$-conjunto infinito qualquer, o qual é não nulo pela letra $(e)$ do Teorema 2.3. Seja $x \in{ }^{*} A-*\langle A\rangle$. Se existir um $\mathbb{R}$-objeto $y$ tal que $x={ }^{*} y$, então teremos ${ }^{*} y \in *^{*} A$ e $y \in A$ pela letra $(c)$ do Corolário 2.5 , resultando em $x={ }^{*} y \in *\langle A\rangle$, o que é absurdo. Portanto, $x$ não está na imagem da função $*$.

Definição 2.6. Dizemos que um ${ }^{*} \mathbb{R}$-objeto é standard se ele está na imagem da função *. Caso contrário, dizemos que ele é não standard.

Exemplo 2.7. Como vimos, se $A$ for um $\mathbb{R}$-conjunto (finito ou infinito), então ${ }^{*} A-*\langle A\rangle$ será o conjunto dos elementos não standard de * $A$. Nesse caso, se $A$ for infinito, então * $A$ terá pelo menos um elemento não standard.

Exemplo 2.8. Seja $\phi\left(x_{1} \ldots x_{n}\right)$ uma condição completamente aberta, e seja $a_{1} \ldots a_{n}$ uma sequência de $\mathbb{R}$-objetos. Todo objeto matemático fixo que aparece na condição fechada 
$\phi\left({ }^{*} a_{1} \ldots{ }^{*} a_{n}\right)$ é standard. Portanto, ao aplicarmos o PT nenhum objeto não standard pode aparecer na ${ }^{*} \mathbb{R}$-condição fechada envolvida.

Teorema 2.9 (Princípio da Definição Standard; PDS - Versão Ingênua). Seja

$$
\phi\left(x_{1} \ldots x_{n}, y_{1} \ldots y_{p}\right)
$$

uma condição completamente aberta, seja $R$ um $\mathbb{R}$-conjunto que é uma relação n-ária, e sejam $a_{1} \ldots a_{p} \mathbb{R}$-objetos. Sejam

$$
C:=\left\{\left(t_{1} \ldots t_{n}\right) \in R: \phi\left(t_{1} \ldots t_{n}, a_{1} \ldots a_{p}\right) \text { é verdadeira }\right\}
$$

$e$

$$
D:=\left\{\left(t_{1} \ldots t_{n}\right) \in{ }^{*} R: \phi\left(t_{1} \ldots t_{n},{ }^{*} a_{1} \ldots{ }^{*} a_{p}\right) \text { é verdadeira }\right\} .
$$

$\operatorname{Temos}^{*} C=D$.

Demonstração. Como $C \subset R$, temos ${ }^{*} C \subset{ }^{*} R$. Nesta seção, provaremos apenas o caso $n=1$, e o caso geral é uma consequência imediata de um princípio geral que veremos na Seção 4.4. A condição fechada

$$
(\forall x \in R)\left(x \in C \Leftrightarrow \phi\left(x, a_{1} \ldots a_{p}\right)\right)
$$

é verdadeira pela definição de $C$, e pelo PT temos que a condição fechada

$$
\left(\forall x \in{ }^{*} R\right)\left(x \in{ }^{*} C \Leftrightarrow \phi\left(x,{ }^{*} a_{1} \ldots{ }^{*} a_{p}\right)\right)
$$

também é verdadeira.

Exemplo 2.10. Considere um intervalo $[a, b]_{\mathbb{R}}$ entre dois números reais $a$ e $b$ com $a \leqslant b$, o qual é definido por

$$
[a, b]_{\mathbb{R}}:=\{x \in \mathbb{R} \vdots a \leqslant x \leqslant b\} .
$$

Pelo PDS, a *-transformação desse intervalo é dada por

$$
\begin{aligned}
{ }^{*}[a, b]_{\mathbb{R}} & =\left\{x \in{ }^{*} \mathbb{R}:{ }^{*} a^{*} \leqslant x^{*} \leqslant{ }^{*} b\right\} \\
& =\left\{x \in{ }^{*} \mathbb{R}: a^{*} \leqslant x^{*} \leqslant b\right\} .
\end{aligned}
$$

\section{Corolário 2.11.}
(a) ${ }^{*} \mathbb{N}=\left\{x \in{ }^{*} \mathbb{Z}: 0 *<x\right\} ;$
(b) ${ }^{*} \mathbb{Z}=\left\{x \in{ }^{*} \mathbb{Q} \vdots x \in{ }^{*} \mathbb{N}\right.$ ou $\left.(-1)^{*} \cdot x \in{ }^{*} \mathbb{N}\right\}$;
(c) ${ }^{*} \mathbb{Q}=\left\{x \in{ }^{*} \mathbb{R} \vdots\left(\exists p, q \in{ }^{*} \mathbb{Z}\right)\left(q \neq 0\right.\right.$ e $\left.\left.x={ }^{*} \div(p, q)\right)\right\}$. 


\subsection{Propriedades Conjuntistas de $*$}

A função * preserva algumas operações básicas da Teoria dos Conjuntos.

Teorema 2.12 (Propriedades Conjuntistas de * - Versão Ingênua). Sejam a e $b$ dois $\mathbb{R}$-objetos, e sejam $A$ e $B$ dois $\mathbb{R}$-conjuntos. Temos:
(a) ${ }^{*}\{a, b\}=\left\{{ }^{*} a,{ }^{*} b\right\}$;
(d) ${ }^{*}(A \cap B)={ }^{*} A \cap{ }^{*} B$
(b) ${ }^{*}(a, b)=\left({ }^{*} a,{ }^{*} b\right)$;
(e) ${ }^{*}(A-B)={ }^{*} A-{ }^{*} B$
(c) ${ }^{*}(A \cup B)={ }^{*} A \cup{ }^{*} B$;
(f) ${ }^{*}(A \times B)={ }^{*} A \times{ }^{*} B$

Demonstração. Sejam $\phi_{1} \ldots \phi_{5}$ as seguintes condições completamente abertas:

- $\phi_{1}\left(x, y_{1}, y_{2}\right):=x=y_{1} \vee x=y_{2}$;

- $\phi_{4}(x, U, V):=x \in U \wedge x \notin V$;

- $\phi_{2}(x, U, V):=x \in U \vee x \in V$;

- $\phi_{3}(x, U, V):=x \in U \wedge x \in V$;

- $\phi_{5}\left(x_{1}, x_{2}, U, V\right):=x_{1} \in U \wedge x_{2} \in V$,

e seja $R$ um $\mathbb{R}$-conjunto que contém os conjuntos $\{a, b\}, A \cup B, A \cap B, A-B$ e $A \times B$. Os resultados das letras $(a),(c),(d),(e)$ e $(f)$ seguem ao aplicarmos o PDS às condições $\phi_{1}, \phi_{2}, \phi_{3}, \phi_{4}$ e $\phi_{5}$, respectivamente. A letra (b) é consequência imediata da $(a)$.

Pela letra $(e)$ do Teorema 2.3, temos que se $A$ for um $\mathbb{R}$-conjunto infinito, então teremos $*\langle A\rangle \subsetneq^{*} A$. A recíproca desse fato também é válida.

Teorema 2.13. Para todo $\mathbb{R}$-conjunto $A$, teremos $*\langle A\rangle \subsetneq^{*} A$ se, e somente se, A for infinito.

Demonstração. Basta provar a condição necessária. Se $A$ for finito, então ele poderá ser escrito na forma $A=\left\{x_{1} \ldots x_{n}\right\}$, onde $x_{1} \ldots x_{n}$ são $\mathbb{R}$-objetos. Nesse caso, pelo Teorema 2.12 , teremos ${ }^{*} A=\left\{{ }^{*} x_{1} \ldots{ }^{*} x_{n}\right\}=*\langle A\rangle$.

Pelo Exemplo 2.7, o Teorema 2.13 pode ser reescrito com as seguintes palavras: * A terá elementos não standard se, e somente se, A for infinito.

A função * preserva algumas noções básicas referentes às relações e funções.

Teorema 2.14 (*-Transformações de Relações e Funções - Versão Ingênua). Sejam A, $B$ e $C$ três $\mathbb{R}$-conjuntos. Se $R \subset A \times B$ e $S \subset C \times A$, então teremos:
(a) ${ }^{*} R \subset{ }^{*} A \times{ }^{*} B$
(c) $\operatorname{Im}\left({ }^{*} R\right)={ }^{*}(\operatorname{Im}(R))$;

(b) $\operatorname{dom}\left({ }^{*} R\right)={ }^{*}(\operatorname{dom}(R))$; 

(d) ${ }^{*} R\left\langle{ }^{*} D\right\rangle={ }^{*}(R\langle D\rangle)(\forall D \subset \operatorname{dom}(R))$;
(f) ${ }^{*} R \circ{ }^{*} S={ }^{*}(R \circ S)$.
(e) $\left({ }^{*} R\right)^{-1}={ }^{*}\left(R^{-1}\right)$;

Em particular, se $f: A \rightarrow B$, então teremos:
(g) $\quad * f:{ }^{*} A \rightarrow{ }^{*} B$;
(i) ${ }^{*} f$ será injetora se, e somente se, $f$ for injetora;
(h) ${ }^{*} f\left({ }^{*} x\right)={ }^{*}(f(x))(\forall x \in A)$;
(j) ${ }^{*} f$ será sobrejetora se, e somente se, $f$ for sobrejetora.

Demonstração. A letra $(a)$ é consequência da letra $(d)$ do Corolário 2.5 e da letra $(f)$ do Teorema 2.12. Seja $M$ um $\mathbb{R}$-conjunto que contém os conjuntos dom $(R), \operatorname{Im}(R), R\langle D\rangle$, $R^{-1}$ e $R \circ S$, e sejam $\phi_{1} \ldots \phi_{5}$ as seguintes condições completamente abertas:

- $\phi_{1}(x, U, T):=(\exists y \in T)(x, y) \in U$;

- $\phi_{2}(x, U, T):=(\exists y \in T)(y, x) \in U$;

- $\phi_{3}(x, U, V):=(\exists y \in V)(y, x) \in U$;

- $\phi_{4}\left(x_{1}, x_{2}, U\right):=\left(x_{2}, x_{1}\right) \in U$;

- $\phi_{5}\left(x_{1}, x_{2}, U, V, T\right):=(\exists y \in T)\left(\left(x_{1}, y\right) \in S \wedge\left(y, x_{2}\right) \in R\right)$.

Pela letra $(d)$ do Corolário 2.5, as *-transformações dos conjuntos dom $(R), \operatorname{Im}(R), R\langle D\rangle$, $R^{-1}$ e $R \circ S$ estão contidas em ${ }^{*} M$. Os resultados das letras $(b),(c),(d),(e)$ e $(f)$ seguem ao aplicarmos o PDS às condições $\phi_{1}, \phi_{2}, \phi_{3}, \phi_{4}$ e $\phi_{5}$, respectivamente, onde a variável $T$ deve ser substituída pelo conjunto $M$.

Sejam $\phi_{6}, \phi_{7}$ e $\phi_{8}$ as seguintes condições completamente abertas:

- $\phi_{6}(U, P, Q):=(\forall x \in P)(\exists ! y \in Q) y=U(x)$;

- $\phi_{7}(U, P, Q):=(\forall x \in P)(\forall y \in Q)(U(x)=U(y) \Rightarrow x=y)$;

- $\phi_{8}(U, P, Q):=(\forall y \in Q)(\exists x \in P) y=U(x)$.

Os resultados das letras $(g),(i)$ e $(j)$ seguem ao aplicarmos o PT às condições $\phi_{6}, \phi_{7}$ e $\phi_{8}$, respectivamente, onde a variável $U$ deve ser substituída pela função $f$, a variável $P$ deve ser substituída pelo conjunto $A$, e a variável $Q$ deve ser substituída pelo conjunto $B$.

Se $\quad x \in A$, então $(x, f(x)) \in f, \quad$ resultando em $\quad\left({ }^{*} x,^{*}(f(x))\right) \in{ }^{*} f, \quad$ e provando $(h)$. 


\subsection{Domínios e Corpos Ordenados Relacionados a * $\mathbb{R}$}

As classes de estruturas dos domínios ordenados e dos corpos ordenados são fechadas sob *-transformações, uma vez que tais classes são axiomatizáveis na lógica de primeira ordem.

\section{Teorema 2.15.}

(a) Se D for um dominio ordenado relacionado a $\mathbb{R}$, então ${ }^{*} D$ será um dominio ordenado quando munido das *-transformações da adição, da multiplicação e da ordem de D;

(b) Se $K$ for um corpo ordenado relacionado a $\mathbb{R}$, então ${ }^{*} K$ será um corpo ordenado quando munido das *-transformações da adição, da multiplicação e da ordem de $K$.

Demonstração.

(a) Os axiomas 1-15 (Seção 1.1) se tornam condições fechadas quando substituímos cada ocorrência dos quantificadores $(\forall x)$ e $(\exists x)$ por $(\forall x \in D)$ e $(\exists x \in D)$, respectivamente. O resultado segue pelo PT.

(b) O axioma (InvM) (Seção 1.2) se torna uma condição fechada quando substituímos cada ocorrência dos quantificadores $(\forall x)$ e $(\exists x)$ por $(\forall x \in K)$ e $(\exists x \in K)$, respectivamente. $\mathrm{O}$ resultado segue pelo $\mathrm{PT}$.

Em particular, o conjunto ${ }^{*} \mathbb{R}$ é um corpo ordenado quando munido das *-transformações da adição, da multiplicação e da ordem de $\mathbb{R}$. O vínculo entre $\mathbb{R}$ e * $\mathbb{R}$ estabelecido pelo Princípio da Transferência faz com que o corpo ordenado dos números hiper-reais se sobressaia às extensões de $\mathbb{R}$ que vimos no Capítulo 1, visto que ele simplifica consideravelmente as demonstrações de vários resultados importantes acerca de $\mathbb{R}$ e ${ }^{*} \mathbb{R}$.

Exemplo 2.16. Seja $D$ um domínio ordenado e seja $K$ um corpo ordenado, ambos relacionados a $\mathbb{R}$, e considere as funções $f: D \rightarrow D$ e $g: K-\{0\} \rightarrow K$ dadas por $f(x):=-x$ e $g(x):=x^{-1}$. As condições

$$
(\forall x \in D) x+f(x)=0
$$

$\mathrm{e}$

$$
(\forall x \in K-\{0\}) x \cdot g(x)=1
$$

são verdadeiras, e, pelo PT, temos que as condições

$$
\left(\forall x \in{ }^{*} D\right) x^{*}+{ }^{*} f(x)=0
$$




$$
\left(\forall x \in{ }^{*} K-\{0\}\right) x^{*} \cdot{ }^{*} g(x)=1
$$

também são verdadeiras. Assim, considerando o domínio ordenado $\left({ }^{*} D,{ }^{*}+,{ }^{*} \cdot, 0,1,{ }^{*}<\right)$ e o corpo ordenado $\left({ }^{*} K,{ }^{*}+,{ }^{*}, 0,1,{ }^{*}<\right)$, temos que se $x \in{ }^{*} D$ e $y \in{ }^{*} K-\{0\}$, então ${ }^{*} f(x)$ e ${ }^{*} g(y)$ serão o inverso aditivo de $x$ em ${ }^{*} D$ e o inverso multiplicativo de $y$ $\mathrm{em}^{*} K$, respectivamente. Por tal razão, denotaremos os respectivos objetos ${ }^{*} f(x)$ e ${ }^{*} g(y)$ simplesmente por $-x$ e $y^{-1}$.

Exemplo 2.17. Todo número real positivo tem uma raiz quadrada, ou seja, a condição

$$
(\forall x \in \mathbb{R})(0<x \Rightarrow(\exists y \in \mathbb{R}) x=y \cdot y)
$$

é verdadeira, e, pelo PT, a condição

$$
\left(\forall x \in{ }^{*} \mathbb{R}\right)\left(0^{*}<x \Rightarrow\left(\exists y \in{ }^{*} \mathbb{R}\right) x=y^{*} \cdot y\right)
$$

é verdadeira. Nota-se que os corpos ordenados $\mathcal{L}$ e $\mathcal{R}(x)$ (Exemplos 1.7 e 1.15) não observam essa propriedade, e, portanto, não são extensões de $\mathbb{R}$ que satisfazem ao Princípio da Transferência.

Os elementos dos conjuntos ${ }^{*} \mathbb{N},{ }^{*} \mathbb{Z},{ }^{*} \mathbb{Q},{ }^{*}$ Irr e ${ }^{*} \mathbb{R}$ são chamados de números hipernaturais, números hiperinteiros, números hiper-racionais, números hiperirracionais e números hiper-reais, respectivamente. Pelo Teorema 2.15 , temos que * $\mathbb{Z}$ é um domínio ordenado enquanto $* \mathbb{Q}$ e $* \mathbb{R}$ são corpos ordenados.

\section{Teorema 2.18.}
(a) $\quad \mathbb{N}=\mathbb{Z} \cap * \mathbb{N}$
(d) ${ }^{*} \mathbb{N}_{\infty}={ }^{*} \mathbb{Z}_{\infty} \cap * \mathbb{N} ;$
(b) $\mathbb{Z}=\mathbb{Q} \cap * \mathbb{Z}$;
(e) ${ }^{*} \mathbb{Z}_{\infty}={ }^{*} \mathbb{Q}_{\infty} \cap * \mathbb{Z} ;$
(c) $\quad \mathbb{Q}=\mathbb{R} \cap * \mathbb{Q} ;$
(f) ${ }^{*} \mathbb{Q}_{\infty}=* \mathbb{R}_{\infty} \cap * \mathbb{Q}$.

Demonstração. As equações das letras $(d),(e)$ e $(f)$ são claras.

(a) Como $\mathbb{N} \subset \mathbb{Z}$ e $\mathbb{N} \subset{ }^{*} \mathbb{N}$, temos $\mathbb{N} \subset \mathbb{Z} \cap{ }^{*} \mathbb{N}$. Se $n \in \mathbb{Z} \cap{ }^{*}$, então $n={ }^{*} n \in{ }^{*} \mathbb{N}$, e, pela letra $(c)$ do Corolário 2.5, teremos $n \in \mathbb{N}$. Provam-se as letras $(b)$ e $(c)$ de modo análogo.

Teorema 2.19. Todo número hipernatural que não é um número natural é infinito. Assim, temos ${ }^{*} \mathbb{N}=\mathbb{N} \cup \mathbb{N}_{\infty} e^{*} \mathbb{Z}=\mathbb{Z} \cup{ }^{*} \mathbb{Z}_{\infty}$, onde tais uniões são disjuntas. 
Demonstração. Suponha que $n \in{ }^{*} \mathbb{N}-\mathbb{N}$ e $n$ é finito. Assim, existe um número natural $m$ tal que $n^{*} \leqslant m$. A condição fechada

$$
(\forall y \in \mathbb{N})(y \leqslant m \Rightarrow(y=1 \vee y=2 \vee \cdots \vee y=m))
$$

é verdadeira, e pelo PT temos que a condição fechada

$$
\left(\forall y \in{ }^{*} \mathbb{N}\right)\left(y^{*} \leqslant m \Rightarrow(y=1 \vee y=2 \vee \cdots \vee y=m)\right)
$$

também é verdadeira, resultando em $n \in\{1,2, \ldots, m\} \subset \mathbb{N}$, o que é absurdo. Se $k \in{ }^{*} \mathbb{Z}-\mathbb{Z}$, então pelo Corolário 2.11 teremos $|k| \in{ }^{*} \mathbb{N}-\mathbb{N}$ e $|k|$ será infinito, resultando em $k \in{ }^{*} \mathbb{Z}_{\infty}$.

Corolário 2.20. O dominio ordenado dos números hiperinteiros, ${ }^{*} \mathbb{Z}$, e os corpos ordenados dos números hiper-racionais e hiper-reais, ${ }^{*} \mathbb{Q} e{ }^{*} \mathbb{R}$, são não arquimedianos.

Demonstração. Pela letra (e) do Teorema 2.3, o conjunto * $\mathbb{Z}$ tem um elemento infinito, e, pela letra $(d)$ do Corolário 2.5 , temos que ${ }^{*} \mathbb{Q}$ e $* \mathbb{R}$ também têm um elemento infinito.

Notação 2.21 (Identificações Usuais). Símbolos que comumente denotam relações n-árias em $\mathbb{R}$ serão usados para denotar as $*$-transformações dessas relações. Assim, se $\diamond$ for um símbolo usual usado (na literatura clássica) para denotar uma relação $R \subset A \times B$ com $A, B \subset \mathbb{R}$, então a relação ${ }^{*} R \subset{ }^{*} A \times{ }^{*} B$ também será denotada por $\diamond$.

Como funções são relações, tal notação também será aplicada para funções. Como exemplo, se $x$ e $y$ forem dois números hiper-reais, então denotaremos a soma $x^{*}+y$ por $x+y$, e se além disso $x$ for positivo e $f:(0, \infty)_{\mathbb{R}} \times \mathbb{R} \rightarrow(0, \infty)_{\mathbb{R}}$ for a função definida por $f(x, y):=x^{y}$, então denotaremos o número hiper-real positivo ${ }^{*} f(x, y)$ por $x^{y}$.

Exemplo 2.22. Empregando a Notação 2.21, o desfecho do Exemplo 2.10 se torna

$$
\begin{aligned}
{ }^{*}[a, b]_{\mathbb{R}} & =\left\{x \in{ }^{*} \mathbb{R}:{ }^{*} a{ }^{*} \leqslant x^{*} \leqslant{ }^{*} b\right\} \\
& =\left\{x \in{ }^{*} \mathbb{R} \vdots a \leqslant x \leqslant b\right\} \\
& =[a, b]_{* \mathbb{R}} .
\end{aligned}
$$

Assim, pelo Teorema 2.15, temos que as $*$-transformações dos intervalos em $\mathbb{R}$ são intervalos em ${ }^{*} \mathbb{R}$ entre números reais.

Dizemos que um número hiperinteiro $n$ é par se $n=2 m\left(\exists m \in{ }^{*} \mathbb{Z}\right)$, e dizemos que $n$ é ímpar se $n=2 m+1\left(\exists m \in{ }^{*} \mathbb{Z}\right)$. Pelo PT, temos que todo número hiperinteiro ou é par ou é ímpar.

Pela Proposição 1.44, os respectivos conjuntos ordenados das galáxias em * $\mathbb{Q}$ e $* \mathbb{R}$ são densos. O mesmo ocorre para o conjunto ordenado das galáxias em * $\mathbb{Z}$. 
Proposição 2.23. O conjunto ordenado das galáxias em ${ }^{*} \mathbb{Z}$ é denso.

Demonstração. Para cada $x \in{ }^{*} \mathbb{Z}$, seja $\mathcal{G}(x)$ a galáxia de $x$ em * $\mathbb{Z}$ (Definição 1.41), e sejam $m$ e $n$ dois números hiperinteiros tais que $\mathcal{G}(m) \triangleleft \mathcal{G}(n)$. Assim, $n-m$ é um número hipernatural infinito. Seja

$$
\begin{aligned}
k & := \begin{cases}m+\frac{n-m}{2} & \text { se } n-m \text { for par; } \\
m+\frac{n-m+1}{2} & \text { se } n-m \text { for ímpar; }\end{cases} \\
& = \begin{cases}\frac{m+n}{2} & \text { se } n-m \text { for par; } \\
\frac{m+n+1}{2} & \text { se } n-m \text { for ímpar. }\end{cases}
\end{aligned}
$$

Nota-se prontamente que $\mathcal{G}(m) \triangleleft \mathcal{G}(k) \triangleleft \mathcal{G}(n)$.

Doravante, usaremos as notações descritas na Definição 1.41 apenas para o caso $D={ }^{*} \mathbb{R}$, ou seja, para cada número hiper-real $x$ teremos

$$
\bar{x}:=x+\operatorname{Inf}\left({ }^{*} \mathbb{R}\right) \quad \text { e } \quad \mathcal{G}(x):=x+\operatorname{Fin}\left({ }^{*} \mathbb{R}\right) .
$$

\subsection{Definição Alternativa para $\mathbb{R}$}

A função $\iota: \mathbb{R} \rightarrow{ }^{*} \mathbb{R}$ dada por $\iota(x):=x$ é claramente uma imersão entre domínios ordenados. Portanto, ${ }^{*} \mathbb{R}$ é uma extensão do domínio ordenado $\mathbb{R}$, e podemos aplicar os resultados da Seção 1.10. No restante desta dissertação, denotaremos a função parte standard associada a * $\mathbb{R}$ por st (Definição 1.81).

Pelo Corolário 1.19 , o conjunto $\operatorname{Fin}\left({ }^{*} \mathbb{Q}\right)$ forma um subdomínio ordenado de $\operatorname{Fin}\left({ }^{*} \mathbb{R}\right)$.

Proposição 2.24. A restrição do morfismo st $:$ Fin $\left({ }^{*} \mathbb{R}\right) \rightarrow \mathbb{R}$ ao subdomínio ordenado Fin $\left({ }^{*} \mathbb{Q}\right)$ de $\operatorname{Fin}\left({ }^{*} \mathbb{R}\right)$ é um morfismo sobrejetor do tipo Fin $\left({ }^{*} \mathbb{Q}\right) \rightarrow \mathbb{R}$ entre domínios não estritamente ordenados cujo kernel é $\operatorname{Inf}\left({ }^{*} \mathbb{Q}\right)$.

Demonstração. Basta provar que a restrição

$$
\text { st }\left\lceil_{\operatorname{Fin}(* \mathbb{Q})}: \operatorname{Fin}\left({ }^{*} \mathbb{Q}\right) \rightarrow \mathbb{R}\right.
$$

é sobrejetora. Seja $r$ um número real e seja $h$ um número hiper-real infinitesimal e positivo. Como a condição fechada

$$
(\forall x \in \mathbb{R})(\forall y \in \mathbb{R})(x<y \Rightarrow(\exists z \in \mathbb{Q})(x<z \wedge z<y))
$$

é verdadeira, temos pelo PT que existe um número hiper-racional $z$ tal que $r<z<r+h$. Assim, $z$ é finito, $z \sim r$, e st $(z)=r$. 
Pelo Teorema 1.40, temos o seguinte corolário.

Corolário 2.25. O quociente

$$
\operatorname{Fin}\left({ }^{*} \mathbb{Q}\right) / \operatorname{Inf}\left({ }^{*} \mathbb{Q}\right)
$$

é isomorfo ao corpo ordenado dos números reais.

Um isomorfismo entre estruturas é uma tradução entre duas maneiras de descrever a essência de um sistema matemático. Assim, pode-se devidamente definir o corpo ordenado dos números reais, $(\mathbb{R},+, \cdot, 0,1,<)$, como sendo o quociente $\operatorname{Fin}\left({ }^{*} \mathbb{Q}\right) / \operatorname{Inf}\left({ }^{*} \mathbb{Q}\right)$. Como veremos no Capítulo 5 , a determinação de ${ }^{*} \mathbb{Q}$ independe de $\mathbb{R}$ e ${ }^{*} \mathbb{R}$, e, portanto, não há circularidade alguma em definir o sistema dos números reais como sendo o quociente mencionado.

Essa descrição do sistema dos números reais prontamente proporciona a ordem usual e as operações de soma e multiplicação no quociente referido, distinguindo-se das construções usuais, as quais exigem algumas deliberações técnicas para definir as munições que tornam o conjunto $\mathbb{R}$ em um corpo ordenado. Além disso, sob a nova definição, provase facilmente que $\mathbb{R}$ satisfaz a diversas propriedades oriundas de $\mathbb{Q}$, como, por exemplo, a Propriedade Arquimediana (Seção 1.3).

Na Seção 2.10, veremos que uma sequência de números racionais, $\{f(n)\}_{n \geqslant 1}$, será uma sequência de Cauchy em $\mathbb{Q}$ se, e somente se, tivermos ${ }^{*} f(M) \sim{ }^{*} f(N)$ para quaisquer números hipernaturais infinitos $M$ e $N$. Nesse caso, como toda sequência de Cauchy é limitada, teremos que ${ }^{*} f(M)$ é um número hiper-racional finito para um $M \in{ }^{*} \mathbb{N}_{\infty}$ qualquer (Corolário 2.47, Letra $(c)$ ), e a mônada

$$
{ }^{*} f(M)+\operatorname{Inf}\left({ }^{*} \mathbb{Q}\right) \in \operatorname{Fin}\left({ }^{*} \mathbb{Q}\right) / \operatorname{Inf}\left({ }^{*} \mathbb{Q}\right)
$$

não dependerá do número hipernatural infinito $M$. Assim, tal mônada determinará um número real.

\subsection{Objetos Internos e Externos}

Postularemos a existência de um método objetivo de rotular os ${ }^{*} \mathbb{R}$-objetos em duas variedades: os objetos internos e os objetos externos. A definição rigorosa dessa noção será apresentada no Capítulo 4, e, para os propósitos deste capítulo, mencionaremos apenas os principais resultados acerca desse tópico.

Intuitivamente, pode-se pensar que os objetos internos se comportam da maneira que esperaríamos que um $\mathbb{R}$-objeto de natureza similar se comportaria, enquanto os objetos externos não acatam às leis que regem a conduta das suas contrapartes relacionadas a $\mathbb{R}$. Como exemplo, temos que todo subconjunto interno de ${ }^{*} \mathbb{R}$ que tem 
uma cota superior tem um supremo em * $\mathbb{R}$, e, como sabemos (Seção 1.7), o mesmo ocorre para os subconjuntos de $\mathbb{R}$ que têm uma cota superior. Todavia, existem subconjuntos de * $\mathbb{R}$ que têm uma cota superior mas não têm um supremo, tal como o conjunto $\mathbb{N}$, e tais objetos são externos.

As provas dos dois teoremas a seguir serão descritas na Seção 4.3.

\section{Teorema 2.26.}

(a) Todo objeto standard é interno;

(b) Todo elemento de um conjunto interno é interno.

A letra (b) do Teorema 2.26 implica que se uma $n$-tupla ordenada $\left(a_{1} \ldots a_{n}\right)$ de ${ }^{*} \mathbb{R}$-objetos for interna, então cada $a_{i}$ será interno.

Teorema 2.27 (Princípio da Definição Interna; PDI - Versão Ingênua). Seja

$$
\phi\left(x_{1} \ldots x_{n}, y_{1} \ldots y_{p}\right)
$$

uma condição completamente aberta, seja $R$ um conjunto interno que é uma relação n-ária, e sejam $a_{1} \ldots a_{p}$ objetos internos. O conjunto

$$
S:=\left\{\left(t_{1} \ldots t_{n}\right) \in R: \phi\left(t_{1} \ldots t_{n}, a_{1} \ldots a_{p}\right) \text { é verdadeira }\right\}
$$

é interno.

Exemplo 2.28. Sejam $a$ e $b$ dois números hiper-reais com $a \leqslant b$. O intervalo $[a, b]_{* \mathbb{R}}$ é definido por

$$
[a, b]_{* \mathbb{R}}:=\left\{x \in{ }^{*} \mathbb{R} \vdots a \leqslant x \leqslant b\right\},
$$

e, como ${ }^{*} \mathbb{R}, a, b$ e $\leqslant$ são objetos internos, temos pelo PDI que o intervalo $[a, b]_{* \mathbb{R}}$ é interno. Analogamente, qualquer intervalo aberto, fechado ou semi-aberto em ${ }^{*} \mathbb{N},{ }^{*} \mathbb{Z},{ }^{*} \mathbb{Q}$ $\mathrm{e}^{*} \mathbb{R}$ é interno.

Corolário 2.29. Seja

$$
\phi\left(x, y, z_{1} \ldots z_{p}\right)
$$

uma condição completamente aberta, seja $R$ um conjunto interno que é uma relação binária, e sejam $a_{1} \ldots a_{p}$ objetos internos. Suponha que para cada $u \in \operatorname{dom}(R)$ existe um único $v \in \operatorname{Im}(R)$ tal que a condição fechada $\phi\left(u, v, a_{1} \ldots a_{p}\right)$ é verdadeira. A função $f$ com dominio dom $(R)$ definida pela condição fechada

$$
\phi\left(u, f(u), a_{1} \ldots a_{p}\right)
$$

é interna. 
Demonstração. Basta notar que

$$
f=\left\{(u, v) \in R \vdots \phi\left(u, v, a_{1} \ldots a_{p}\right) \text { é verdadeira }\right\}
$$

e aplicar o PDI.

O atributo de um conjunto ser interno é preservado sobre algumas operações conjuntistas.

Teorema 2.30 (Propriedades Conjuntistas dos Objetos Internos - Versão Ingênua).

(a) Se a e b forem objetos internos, então o conjunto $\{a, b\}$ será interno;

(b) Se $A$ e $B$ forem conjuntos internos, então os conjuntos

$$
A \cup B, A \cap B, A-B \text { e } A \times B
$$

serão internos;

(c) Se $R$ for uma relação interna, e se $A$ for um conjunto interno, entãa $R^{-1}$, $\operatorname{dom}(R)$ e $R\langle A\rangle$ serão internos.

Demonstração. Sejam $\phi_{1} \phi_{2} \ldots \phi_{8}$ as seguintes condições completamente abertas:

- $\phi_{1}\left(x, y_{1}, y_{2}\right):=x=y_{1} \vee x=y_{2}$

- $\phi_{2}(x, U, V):=x \in U \vee x \in V$;

- $\phi_{3}(x, U, V):=x \in U \wedge x \in V$;

- $\phi_{4}(x, U, V):=x \in U \wedge x \notin V$;
- $\phi_{5}\left(x_{1}, x_{2}, U, V\right):=x_{1} \in U \wedge x_{2} \in V$

- $\phi_{6}\left(x_{1}, x_{2}, U\right):=\left(x_{2}, x_{1}\right) \in U$;

- $\phi_{7}(x, U, T):=(\exists y \in T)(x, y) \in U$;

- $\phi_{8}(x, U, V):=(\exists y \in V)(y, x) \in U$.

O resultado da letra $(a)$ segue ao aplicarmos o PDI à condição $\phi_{1}$, e os resultados da letra (b) seguem ao aplicarmos o PDI às condições $\phi_{2}, \phi_{3}, \phi_{4}$ e $\phi_{5}$.

(c) Aplicando o PDI às condições $\phi_{6}$ e $\phi_{8}$, obtém-se facilmente que $R^{-1}$ e $R\langle A\rangle$ são internos. Assuma que existe ${ }^{1}$ um conjunto interno $B$ que contém dom $(R)$. Assim, obtém-se que o conjunto dom $(R)$ é interno aplicando o PDI à condição $\phi_{7}$, onde as respectivas variáveis $U$ e $T$ devem ser substituídas por $R$ e $B$.

1 Não é possível confirmar a existência desse conjunto neste capítulo. Na Seção 4.3, veremos que o aprovisionamento dessa peça imprescindível para a presente demonstração é completamente justificável. 
Denotaremos o conjunto dos objetos internos por $\mathbb{I}$.

O teorema a seguir será provado na Seção 4.4.

Teorema 2.31. Sejam $A$ e $B$ dois $\mathbb{R}$-conjuntos. Temos:
(a) ${ }^{*} \mathcal{P}^{n}(A)=\mathbb{I} \cap \mathcal{P}^{n}\left({ }^{*} A\right)$, onde $n \in \mathbb{N}$;
(c) $\quad *(\operatorname{Int}(A, \leqslant) B)=\mathbb{I} \cap \operatorname{Int}\left({ }^{*} A,{ }^{*} \leqslant\right)\left({ }^{*} B\right)$ onde
(b) $\quad *\left({ }^{A} B\right)=\mathbb{I} \cap\left({ }^{*} A\right)\left({ }^{*} B\right)$;
$\leqslant$ é uma relação binária em $A$.

Exemplo 2.32. Pelo Princípio da Boa Ordenação (Teorema B.12), a condição fechada

$$
(\forall S \in \mathcal{P}(\mathbb{N})-\{\emptyset\})(\exists x \in S)(\forall y \in S) x \leqslant y
$$

é verdadeira. Pelo PT e pela letra $(a)$ do Teorema 2.31, todo subconjunto interno não nulo de ${ }^{*} \mathbb{N}$ tem um menor elemento. Analogamente, como todo subconjunto não nulo de $\mathbb{N}$ que é limitado superiormente tem um maior elemento, temos que todo subconjunto interno não nulo de ${ }^{*} \mathbb{N}$ que é limitado superiormente tem um maior elemento.

Suponha que o conjunto dos números naturais é interno. Assim, a diferença ${ }^{*} \mathbb{N}-\mathbb{N}={ }^{*} \mathbb{N}_{\infty}$ é interna (Teorema 2.30, Letra $(b)$ ), e, como ela é não nula (Teorema 2.3, Letra (e)), ela tem um menor elemento $N$. Como $N$ é infinito, temos $n<N(\forall n \in \mathbb{N})$, resultando em $n<N-1(\forall n \in \mathbb{N})$ e $N-1 \in{ }^{*} \mathbb{N}_{\infty}$, o que é absurdo visto que $N-1<N$. Portanto, o conjunto $\mathbb{N}$ é externo.

Exemplo 2.33. Pelo Princípio da Indução (Exemplo B.20), a condição fechada

$$
(\forall S \in \mathcal{P}(\mathbb{N}))[(0 \in S \wedge(\forall n \in \mathbb{N})(n \in S \rightarrow n+1 \in S)) \rightarrow S=\mathbb{N}]
$$

é verdadeira, resultando pelo PT que se $S$ for um conjunto interno de números hipernaturais que contém o número 0 , e se o sucessor de cada elemento de $S$ pertencer a $S$, então $S$ será igual ao conjunto ${ }^{*} \mathbb{N}$. Esse resultado é conhecido como Princípio da Indução Interna.

Exemplo 2.34. Como ${ }^{*} \mathbb{N}$ é standard, temos que ${ }^{*} \mathbb{N}$ é interno. Já o conjunto das partes $\mathcal{P}\left({ }^{*} \mathbb{N}\right)$ não pode ser interno, pois caso contrário o elemento $\mathbb{N} \in \mathcal{P}\left({ }^{*} \mathbb{N}\right)$ também seria interno (Teorema 2.26, Letra (b)), o que é absurdo (Exemplo 2.32). Assim, o conjunto das partes de um conjunto interno não é necessariamente interno.

Corolário 2.35. Se A for um $\mathbb{R}$-conjunto infinito, então $*\langle A\rangle$ será externo.

Demonstração. Seja $f: \mathbb{N} \rightarrow A$ injetora. Se $*\langle A\rangle$ for interno, então o conjunto

$$
*\langle A\rangle \cap *(f\langle\mathbb{N}\rangle)=*\langle f\langle\mathbb{N}\rangle\rangle={ }^{*} f\langle\mathbb{N}\rangle
$$

será interno, e, como ${ }^{*} f$ é injetora (Teorema 2.14, Letra $(i)$ ), teremos que $\left({ }^{*} f\right)^{-1}\left\langle{ }^{*} f\langle\mathbb{N}\rangle\right\rangle=\mathbb{N}$ é interno, o que é absurdo (Exemplo 2.32). 
Exemplo 2.36. Seja $\mathcal{D}: \operatorname{Int}(\mathbb{Z},<) \mathbb{R} \rightarrow \mathcal{P}(\mathbb{Z})$ a função definida por $\mathcal{D}(x):=\operatorname{dom}(x)$. Assim, a condição fechada

$$
(\forall x \in \operatorname{Int}(\mathbb{Z},<) \mathbb{R})(\forall n \in \mathbb{Z})(n \in \mathcal{D}(x) \Leftrightarrow(\exists r \in \mathbb{R})(n, r) \in x)
$$

é verdadeira, e pelo PT e pelo Teorema 2.31 temos que a condição

$$
\left(\forall x \in \mathbb{I} \cap \operatorname{Int}\left({ }^{*} \mathbb{Z}^{*}<\right)\left({ }^{*} \mathbb{R}\right)\right)\left(\forall n \in{ }^{*} \mathbb{Z}\right)\left(n \in{ }^{*} \mathcal{D}(x) \Leftrightarrow\left(\exists r \in{ }^{*} \mathbb{R}\right)(n, r) \in x\right)
$$

também é verdadeira, resultando que a função ${ }^{*} \mathcal{D}: \mathbb{I} \cap \operatorname{Int}\left({ }^{*} \mathbb{Z},{ }^{*}<\right)\left({ }^{*} \mathbb{R}\right) \rightarrow \mathbb{I} \cap \mathcal{P}\left({ }^{*} \mathbb{Z}\right)$ é tal que

$$
{ }^{*} \mathcal{D}(x)=\operatorname{dom}(x)
$$

para todo $x \in \mathbb{I} \cap \operatorname{Int}\left({ }^{*} \mathbb{Z},{ }^{*}<\right)\left({ }^{*} \mathbb{R}\right)$.

Exemplo 2.37. Denotaremos por $\mathcal{P}_{\text {sup }}(\mathbb{R})$ o conjunto dos subconjuntos de $\mathbb{R}$ que têm um supremo, ou seja

$$
\begin{aligned}
\mathcal{P}_{\text {sup }}(\mathbb{R}) & :=\{S \subset \mathbb{R} \vdots S \text { tem um supremo }\} \\
& =\{S \in \mathcal{P}(\mathbb{R}) \vdots(\exists M \in \mathbb{R})((\forall x \in S) x \leqslant M \wedge(\forall N \in \mathbb{R})((\forall x \in S) x \leqslant N \Rightarrow M \leqslant N))\}
\end{aligned}
$$

Pelo PDS e pelo Teorema 2.31, temos

$$
\begin{aligned}
{ }^{*} \mathcal{P}_{\text {sup }}(\mathbb{R}) & =\left\{S \in \mathbb{I} \cap \mathcal{P}\left({ }^{*} \mathbb{R}\right) \vdots\left(\exists M \in{ }^{*} \mathbb{R}\right)\left((\forall x \in S) x \leqslant M \wedge\left(\forall N \in{ }^{*} \mathbb{R}\right)((\forall x \in S) x \leqslant N \Rightarrow M \leqslant N)\right)\right\} \\
& =\left\{S \subset{ }^{*} \mathbb{R} \vdots S \text { é interno e tem um supremo }\right\} .
\end{aligned}
$$

Denotaremos por $\mathcal{P}_{\text {inf }}(\mathbb{R})$ o conjunto dos subconjuntos de $\mathbb{R}$ que têm um ínfimo. Analogamente ao procedimento que vimos, obtém-se que ${ }^{*} \mathcal{P}_{\text {inf }}(\mathbb{R})$ é o conjunto dos subconjuntos internos de ${ }^{*} \mathbb{R}$ que têm um ínfimo.

Exemplo 2.38. Sejam Sup : $\mathcal{P}_{\text {sup }}(\mathbb{R}) \rightarrow \mathbb{R}$ e $\operatorname{lnf}: \mathcal{P}_{\text {inf }}(\mathbb{R}) \rightarrow \mathbb{R}$ as funções respectivamente definidas por

$$
\operatorname{Sup}(S):=\sup (S) \text { e } \operatorname{lnf}(S):=\inf (S) .
$$

Assim sendo, temos que as condições fechadas

$$
\left(\forall S \in \mathcal{P}_{\text {sup }}(\mathbb{R})\right)((\forall x \in S) x \leqslant \operatorname{Sup}(S) \wedge(\forall N \in \mathbb{R})((\forall x \in S) x \leqslant N \Rightarrow \operatorname{Sup}(S) \leqslant N))
$$

e

$$
\left(\forall S \in \mathcal{P}_{\text {inf }}(\mathbb{R})\right)((\forall x \in S) \operatorname{lnf}(S) \leqslant x \wedge(\forall N \in \mathbb{R})((\forall x \in S) N \leqslant x \Rightarrow N \leqslant \operatorname{lnf}(S)))
$$

são verdadeiras, e pelo PT temos que as condições

$$
\left(\forall S \in{ }^{*} \mathcal{P}_{\text {sup }}(\mathbb{R})\right)\left((\forall x \in S) x \leqslant{ }^{*} \operatorname{Sup}(S) \wedge\left(\forall N \in{ }^{*} \mathbb{R}\right)\left((\forall x \in S) x \leqslant N \Rightarrow{ }^{*} \operatorname{Sup}(S) \leqslant N\right)\right)
$$


$\left(\forall S \in{ }^{*} \mathcal{P}_{\text {inf }}(\mathbb{R})\right)\left((\forall x \in S){ }^{*} \operatorname{Inf}(S) \leqslant x \wedge\left(\forall N \in{ }^{*} \mathbb{R}\right)\left((\forall x \in S) N \leqslant x \Rightarrow N \leqslant{ }^{*} \operatorname{Inf}(S)\right)\right)$

são verdadeiras. Portanto, as funções ${ }^{*} \operatorname{Sup}:{ }^{*} \mathcal{P}_{\text {sup }}(\mathbb{R}) \rightarrow{ }^{*} \mathbb{R}$ e ${ }^{*} \operatorname{Inf}:{ }^{*} \mathcal{P}_{\text {inf }}(\mathbb{R}) \rightarrow{ }^{*} \mathbb{R}$ são tais que

$$
{ }^{*} \operatorname{Sup}\left(S_{1}\right)=\sup \left(S_{1}\right) \text { e }{ }^{*} \operatorname{lnf}\left(S_{2}\right)=\inf \left(S_{2}\right)
$$

para quaisquer subconjuntos internos $S_{1}$ e $S_{2}$ de ${ }^{*} \mathbb{R}$ tais que $S_{1}$ tem um supremo e $S_{2}$ tem um ínfimo.

Corolário 2.39. Os conjuntos numéricos

$$
\begin{gathered}
\mathbb{N}, \mathbb{Z}, \mathbb{Q}, \operatorname{Irr}, \mathbb{R}, \operatorname{Inf}\left({ }^{*} \mathbb{Q}\right), \operatorname{Inf}\left({ }^{*} \operatorname{Irr}\right), \operatorname{Inf}\left({ }^{*} \mathbb{R}\right), \operatorname{Fin}\left({ }^{*} \mathbb{Q}\right), \\
\operatorname{Fin}\left({ }^{*} \operatorname{Irr}\right), \operatorname{Fin}\left({ }^{*} \mathbb{R}\right),{ }^{*} \mathbb{N}_{\infty},{ }^{*} \mathbb{Z}_{\infty},{ }^{*} \mathbb{Q}_{\infty},{ }^{*} \operatorname{Ir} r_{\infty} e^{*} \mathbb{R}_{\infty}
\end{gathered}
$$

são externos.

Demonstração. Vimos que $\mathbb{N}$ e ${ }^{*} \mathbb{N}_{\infty}$ são externos (Exemplo 2.32), e, como os conjuntos infinitos $\mathbb{Z}, \mathbb{Q}, \operatorname{Irr}$ e $\mathbb{R}$ são da forma $*\langle A\rangle$ com $A \subset \mathbb{R}$ infinito, pelo Corolário 2.35 temos que eles são externos.

O corpo ordenado dos números reais satisfaz à Propriedade do Supremo (Seção 1.7), ou seja, a condição fechada abreviada

$$
\begin{aligned}
(\forall S \in \mathcal{P}(\mathbb{R}))(S \neq \emptyset \wedge(\exists x \in \mathbb{R})(" x \text { é uma cota superior de } S ") \\
\quad \Rightarrow(\exists y \in \mathbb{R}) \text { (" } y \text { é o supremo de } S "))
\end{aligned}
$$

é verdadeira. Portanto, pelo PT e pelo Teorema 2.31, a condição fechada

$$
\left(\forall S \in \mathbb{I} \cap \mathcal{P}\left({ }^{*} \mathbb{R}\right)\right)\left(S \neq \emptyset \wedge\left(\exists x \in{ }^{*} \mathbb{R}\right)\left(" x \text { é uma cota superior de } S^{\prime \prime}\right)\right.
$$

$$
\left.\left.\Rightarrow\left(\exists y \in{ }^{*} \mathbb{R}\right) \text { (" } y \text { é o supremo de } S^{\prime \prime}\right)\right)
$$

é verdadeira, e os conjuntos $\operatorname{Inf}\left({ }^{*} \mathbb{Q}\right), \operatorname{Inf}\left({ }^{*} \operatorname{Irr}\right), \operatorname{Inf}\left({ }^{*} \mathbb{R}\right), \operatorname{Fin}\left({ }^{*} \mathbb{Q}\right), \operatorname{Fin}\left({ }^{*} \operatorname{Irr}\right)$ e $\operatorname{Fin}\left({ }^{*} \mathbb{R}\right)$ não podem ser internos, visto que todos eles têm uma cota superior em * $\mathbb{R}$ e nenhum deles tem um supremo.

Os conjuntos ${ }^{*} \mathbb{N},{ }^{*} \mathbb{Z}$ e ${ }^{*} \mathbb{Q}$ são standard, e, portanto, são internos. Como temos

$$
\begin{aligned}
& { }^{*} \mathbb{N}_{\infty}={ }^{*} \mathbb{N} \cap{ }^{*} \mathbb{Z}_{\infty} ; \\
& { }^{*} \mathbb{Z}_{\infty}={ }^{*} \mathbb{Z} \cap{ }^{*} \mathbb{Q}_{\infty} ; \\
& { }^{*} \mathbb{Q}_{\infty}={ }^{*} \mathbb{Q} \cap{ }^{*} \mathbb{R}_{\infty},
\end{aligned}
$$

pela letra (b) do Teorema 2.30 temos que os conjuntos ${ }^{*} \mathbb{Z}_{\infty},{ }^{*} \mathbb{Q}_{\infty}$ e ${ }^{*} \mathbb{R}_{\infty}$ são externos. 


\subsection{Aplicações no Cálculo Diferencial}

O Princípio da Transferência permite que as noções básicas do Cálculo Diferencial possam ser reescritas em termos dos números hiper-reais, e essas novas perspectivas muito se assemelham às interpretações que uma série de matemáticos dos séculos XVII e XVIII nutriam acerca desses conceitos.

O Teorema 2.41, o Teorema 2.46 e o Corolário 2.47 desta seção apresentam algumas dessas traduções dos conceitos do Cálculo para a Análise Não Standard. Antes de enunciarmos tais resultados, introduziremos a seguinte notação:

Notação 2.40. Sejam $I$ e $R$ dois conjuntos relacionados a $\mathbb{R}$, e seja $\left\{x_{i}\right\}_{i \in I}$ uma família de elementos de $R$ com índices em $I$. Se $j$ for um elemento de ${ }^{*} I$, então denotaremos por $x_{j}$ o elemento ${ }^{*} f(j)$ do conjunto ${ }^{*} R$, onde $f$ é a função $I \rightarrow R$ definida por $f(i):=x_{i}$. No caso $I=\left[n_{0}, \infty\right)_{\mathbb{Z}}$ para algum $n_{0} \in \mathbb{Z}$, dizemos que a família

$$
\left\{{ }^{*} f(i)\right\}_{i \in \in^{*} I}=\left\{{ }^{*} f(n)\right\}_{\substack{n \geqslant n_{0} \\ n \in *^{*}}}
$$

é uma hipersequência de elementos de ${ }^{*} R$.

Em outras palavras, a Notação 2.40 estipula que sempre que um subíndice em um termo geral de uma família pertencer à *-transformação do conjunto de índices original, estará pressuposto que, de fato, trata-se de um termo da *-transformação daquela família.

Teorema 2.41 (Equivalências no Cálculo). Seja I um conjunto de números reais e seja $i_{0}$ um número real estendido. ${ }^{2}$ Temos:

(a) I será limitado superiormente se, e somente se, todo elemento positivo de * I for finito;

(b) $i_{0}$ será um ponto de acumulação ${ }^{3}$ de I pela esquerda se, e somente se, tivermos $i \sim i_{0}$ para algum $i \in{ }^{*} I$ menor que $i_{0}$.

Suponha que $i_{0}$ é um ponto de acumulação de I pela esquerda. Seja $S$ um conjunto relacionado a $\mathbb{R}$, seja $\left\{f_{i}\right\}_{i \in I}$ uma família de funções reais definidas ${ }^{4}$ em $S$ com indices em $I$, e seja $f$ uma função real definida em $S$.

2 O conjunto ordenado dos números reais estendidos consiste no conjunto $\overline{\mathbb{R}}:=\mathbb{R} \cup\{-\infty, \infty\}$, onde $-\infty$ e $\infty$ são objetos que não pertencem a $\mathbb{R}$, munido da extensão da ordem usual de $\mathbb{R}$ definida por $-\infty<\infty$ e $-\infty<x<\infty(\forall x \in \mathbb{R})$. Dizemos que $-\infty$ e $\infty$ são os elementos infinitos de $\overline{\mathbb{R}}$. A condição $0<x \in{ }^{*} \mathbb{R}_{\infty}$ (resp. $0>x \in{ }^{*} \mathbb{R}_{\infty}$ ) é denotada por $x \sim \infty$ (resp. $x \sim-\infty$ ), e o conjunto $\left\{x \in{ }^{*} \mathbb{R} \vdots x \sim \infty\right\}$ (resp. $\left.\left\{x \in{ }^{*} \mathbb{R} \vdots x \sim-\infty\right\}\right)$ é denotado por $\bar{\infty}$ (resp. $\overline{-\infty}$ ). Note que as condições $x \sim \infty$ e $y \sim \infty$ não implicam $x \sim y$.

3 Estamos considerando que o elemento $\infty$ (resp. $-\infty$ ) do conjunto ordenado dos números reais estendidos, $\overline{\mathbb{R}}=\mathbb{R} \cup\{-\infty, \infty\}$, é um ponto de acumulação pela esquerda (resp. pela direita) dos subconjuntos de $\mathbb{R}$ que são ilimitados superiormente (resp. inferiormente). Analogamente, consideraremos que $\infty$ (resp. $-\infty$ ) é um ponto de acumulação das funções reais que são ilimitadas superiormente (resp. inferiormente) nas proximidades de um ponto de acumulação do domínio.

4 Dizemos que uma função $f$ é definida em $S$ se $S \subset \operatorname{dom}(f)$. 
(c) $\lim _{i \rightarrow i_{0}^{-}} f_{i}=f$ pontualmente em $S$ se, e somente se, $f_{i}(x) \sim^{*} f(x)$ para todo $i \in \overline{i_{0}} \cap^{*} I$ menor que $i_{0}$ e para todo $x \in *\langle S\rangle$;

(d) $\lim _{i \rightarrow i_{0}^{-}} f_{i}=f$ uniformemente em $S$ se, e somente se, $f_{i}(x) \sim^{*} f(x)$ para todo $i \in \overline{i_{0}} \cap^{*} I$ menor que $i_{0}$ e para todo $x \in{ }^{*} S$.

Suponha que $S \subset \mathbb{R}$ e $x_{0}$ é um ponto de acumulação de $S$ pela esquerda. Seja l um número real estendido.

(e) $f$ será uniformemente contínua em $S$ se, e somente se, ${ }^{*} f(x) \sim{ }^{*} f(y)$ para quaisquer $x, y \in{ }^{*} S \operatorname{com} x \sim y$;

(f) $\quad \lim _{\substack{x \rightarrow x_{0}^{-} \\ x \in S}} f(x)=l$ se, e somente se, ${ }^{*} f(x) \sim l$ para todo $x \in \overline{x_{0}} \cap{ }^{*} S$ menor que $x_{0}$;

(g) $f$ será uma função de Cauchy nas proximidades de $x_{0}$ em $S$ pela esquerda se, e somente se, ${ }^{*} f(x) \sim{ }^{*} f(y)$ para quaisquer $x, y \in \overline{x_{0}} \cap{ }^{*} S$ menores que $x_{0}$;

(h) l será um ponto de acumulação de $f$ nas proximidades de $x_{0}$ em $S$ pela esquerda se, e somente se, ${ }^{*} f(x) \sim l$ para algum $x \in \overline{x_{0}} \cap{ }^{*} S$ menor que $x_{0}$;

(i) $f$ será limitada superiormente nas proximidades de $x_{0}$ em $S$ pela esquerda se, e somente se, existir um número real $M$ tal que ${ }^{*} f(x)<M$ para todo $x \in \overline{x_{0}} \cap{ }^{*} S$ menor que $x_{0}$.

Suponha que $x_{0} \in S$.

(j) f terá um máximo local nas proximidades de $x_{0}$ em $S$ pela esquerda se, e somente se, ${ }^{*} f(x) \leqslant f\left(x_{0}\right)$ para todo $x \in \overline{x_{0}} \cap{ }^{*} S$ menor que $x_{0}$;

(k) $f$ será contínua em $x_{0}$ pela esquerda se, e somente se, ${ }^{*} f(x) \sim f\left(x_{0}\right)$ para todo $x \in \overline{x_{0}} \cap{ }^{*} S$ menor que $x_{0}$.

Seja b um número real.

(1) f será diferenciável em $x_{0}$ pela esquerda com derivada b se, e somente se, tivermos

$$
\frac{* f(x)-f\left(x_{0}\right)}{x-x_{0}} \sim b
$$

para todo $x \in \overline{x_{0}} \cap * S$ menor que $x_{0}$.

Demonstração. Provaremos tais afirmações apenas para o caso em que os números reais estendidos $i_{0}, l$ e $x_{0}$ são finitos, e as provas dos casos em que $i_{0}, l$ ou $x_{0}$ são números reais estendidos infinitos são análogas às que veremos aqui. 
(a) Se $I$ for limitado superiormente, então existirá um número real positivo $r$ tal que $i<r(\forall i \in I)$, e, pelo PT, teremos $i<r\left(\forall i \in{ }^{*} I\right)$, implicando na condição necessária da afirmação. Reciprocamente, se todo elemento positivo de ${ }^{*} I$ for finito, então a condição fechada

$$
\left(\exists r \in{ }^{*} \mathbb{R}\right)\left(0<r \wedge\left(\forall i \in{ }^{*} I\right) i<r\right)
$$

será verdadeira (pois bastaria escolher um $r$ infinito e positivo), e o resultado seguirá ao aplicarmos o PT a essa * $\mathbb{R}$-condição.

(b) Suponha que $i_{0}$ é um ponto de acumulação de $I$ pela esquerda. Isso significa que a condição fechada

$$
(\forall \epsilon \in \mathbb{R})\left(0<\epsilon \Rightarrow(\exists i \in I) 0<i_{0}-i<\epsilon\right)
$$

é verdadeira, e, pelo PT, se $\epsilon$ for um número hiper-real positivo e infinitesimal, então existirá um $i \in{ }^{*} I$ tal que $0<i_{0}-i<\epsilon$, resultando em $i \sim i_{0}$ e $i<i_{0}$. Reciprocamente, se $i \sim i_{0}$ para algum $i \in{ }^{*} I$ menor que $i_{0}$, se $\epsilon$ for um número real positivo, então teremos $0<i_{0}-i<\epsilon$, e a condição fechada

$$
\left(\exists i \in{ }^{*} I\right) 0<i_{0}-i<\epsilon
$$

será verdadeira. Nesse caso, pelo PT, existirá um $i \in I$ com $0<i_{0}-i<\epsilon$, resultando que $i_{0}$ será um ponto de acumulação de $I$ pela esquerda.

(d) Suponha que $\lim _{i \rightarrow i_{0}^{-}} f_{i}=f$ uniformemente em $S$. Seja $\epsilon$ um número real positivo, e seja $\delta_{\epsilon}$ um número real positivo tal que a condição fechada

$$
(\forall i \in I)\left(0<i_{0}-i<\delta_{\epsilon} \Rightarrow(\forall x \in S)\left|f_{i}(x)-f(x)\right|<\epsilon\right)
$$

é verdadeira. Se $i \in \overline{i_{0}} \cap{ }^{*} I$ com $i<i_{0}$, e se $x \in{ }^{*} S$, então teremos $0<i_{0}-i<\delta_{\epsilon}$, e, pelo PT, teremos $\left|f_{i}(x)-{ }^{*} f(x)\right|<\epsilon$, implicando em $f_{i}(x) \sim{ }^{*} f(x)$ pois $\epsilon$ é arbitrário. Reciprocamente, se $f_{i}(x) \sim^{*} f(x)$ para todo $i \in \overline{i_{0}} \cap^{*} I$ menor que $i_{0}$ e para todo $x \in{ }^{*} S$, e se $\epsilon$ for um número real positivo, então a condição fechada

$$
\left(\exists \delta \in{ }^{*} \mathbb{R}\right)\left(0<\delta \wedge\left(\forall i \in{ }^{*} I\right)\left(0<i_{0}-i<\delta \Rightarrow\left(\forall x \in{ }^{*} S\right)\left|f_{i}(x)-{ }^{*} f(x)\right|<\epsilon\right)\right)
$$

será verdadeira (pois bastaria escolher um $\delta$ infinitesimal e positivo), e o resultado seguirá ao aplicarmos o PT a essa *R-condição.

(e) Suponha que $f$ é uniformemente contínua em $S$. Seja $\epsilon$ um número real positivo qualquer, e seja $\delta_{\epsilon}$ um número real positivo tal que a condição fechada

$$
(\forall x, y \in S)\left(|x-y|<\delta_{\epsilon} \Rightarrow|f(x)-f(y)|<\epsilon\right)
$$


é verdadeira. Se $x, y \in{ }^{*} S \operatorname{com} x \sim y$, então $|x-y|<\delta_{\epsilon}$, e pelo PT teremos $\left|{ }^{*} f(x)-{ }^{*} f(y)\right|<\epsilon$, resultando em ${ }^{*} f(x) \sim{ }^{*} f(y)$ pois $\epsilon$ é arbitrário. Reciprocamente, se ${ }^{*} f(x) \sim^{*} f(y)$ para quaisquer $x, y \in{ }^{*} S$ com $x \sim y$, e se $\epsilon$ for um número real positivo, então a condição fechada

$$
\left(\exists \delta \in{ }^{*} \mathbb{R}\right)\left(0<\delta \wedge\left(\forall x, y \in{ }^{*} S\right)\left(|x-y|<\left.\delta_{\epsilon} \Rightarrow\right|^{*} f(x)-{ }^{*} f(y) \mid<\epsilon\right)\right)
$$

será verdadeira (pois bastaria escolher um $\delta$ infinitesimal e positivo), e o resultado seguirá ao aplicarmos o PT a essa * R-condição.

(f) Suponha que o limite $\lim _{\substack{x \rightarrow x_{0}^{-} \\ x \in S}} f(x)$ existe e é igual a $l$. Seja $\epsilon$ um número real positivo qualquer, e seja $\delta_{\epsilon}$ um número real positivo tal que a condição fechada

$$
(\forall x \in S)\left(0<x_{0}-x<\delta_{\epsilon} \Rightarrow|f(x)-l|<\epsilon\right)
$$

é verdadeira. Se $x \in \overline{x_{0}} \cap{ }^{*} S \operatorname{com} x<x_{0}$, então teremos $0<x_{0}-x<\delta_{\epsilon}$, e, pelo PT, teremos $\left|{ }^{*} f(x)-l\right|<\epsilon$, implicando em ${ }^{*} f(x) \sim l$ pois $\epsilon$ é arbitrário. Reciprocamente, se ${ }^{*} f(x) \sim l$ para todo $x \in \overline{x_{0}} \cap{ }^{*} S$ menor que $x_{0}$, e se $\epsilon$ for um número real positivo, então a condição fechada

$$
\left(\exists \delta \in{ }^{*} \mathbb{R}\right)\left(0<\delta \wedge\left(\forall x \in{ }^{*} S\right)\left(0<x_{0}-x<\left.\delta \Rightarrow\right|^{*} f(x)-l \mid<\epsilon\right)\right)
$$

será verdadeira (pois bastaria escolher um $\delta$ infinitesimal e positivo), e o resultado seguirá ao aplicarmos o $\mathrm{PT}^{5}$ a essa * $\mathbb{R}$-condição.

(c) Se $r \in S$ e $x={ }^{*} r$, então, como $f(r) \in \mathbb{R}$, teremos (Teorema 2.14, Letra $(h)$ )

$$
{ }^{*} f(x)={ }^{*} f\left({ }^{*} r\right)={ }^{*}(f(r))=f(r) .
$$

O resultado segue ao aplicarmos a letra $(f)$;

(g) Suponha que $f$ é uma função de Cauchy nas proximidades de $x_{0}$ em $S$ pela esquerda. Seja $\epsilon$ um número real positivo qualquer, e seja $x_{\epsilon}$ um elemento de $S$ menor que $x_{0}$ tal que a condição fechada

$$
(\forall x, y \in S)\left(\left(x_{\epsilon}<x<x_{0} \wedge x_{\epsilon}<y<x_{0}\right) \Rightarrow|f(x)-f(y)|<\epsilon\right)
$$

5 O leitor deve estar atento à Notação 2.21. Na ausência das convenções estabelecidas nessa notação, a * $\mathbb{R}$-condição fechada a qual nos referimos na prova da letra $(f)$ é expressa como

$$
\left(\exists \delta \in{ }^{*} \mathbb{R}\right)\left(0^{*}<\delta \wedge\left(\forall x \in{ }^{*} S\right)\left(0^{*}<{ }^{*} M\left(x^{*}-a\right){ }^{*}<\delta \Rightarrow{ }^{*} M\left({ }^{*} f(x){ }^{*}-l\right){ }^{*}<\epsilon\right)\right),
$$

onde $M: \mathbb{R} \rightarrow \mathbb{R}$ é a função valor absoluto dada por $M(x):=|x|$, e, tendo em vista que ${ }^{*} x=x(\forall x \in \mathbb{R})$, tal condição também pode ser exprimida como

$\left(\exists \delta \in{ }^{*} \mathbb{R}\right)\left({ }^{*} 0{ }^{*}<\delta \wedge\left(\forall x \in{ }^{*} S\right)\left({ }^{*} 0^{*}<{ }^{*} M\left(x^{*}-{ }^{*} a\right){ }^{*}<\delta \Rightarrow{ }^{*} M\left({ }^{*} f(x){ }^{*}-{ }^{*} l\right){ }^{*}<{ }^{*} \epsilon\right)\right)$,

evidenciando o fato de que todo objeto matemático fixo que aparece nessa condição é um objeto standard (Exemplo 2.8) e facilitando o entendimento da aplicação do PT a essa condição. A inconveniência nítida de abandonar a Notação 2.21 consiste na abundância de asteriscos que ocorrem nas $* \mathbb{R}$-condições fechadas. 
é verdadeira. Se $x, y \in \overline{x_{0}} \cap{ }^{*} S$ com $x, y<x_{0}$, então teremos $x_{\epsilon}<x$ e $x_{\epsilon}<y$, resultando pelo PT em $|f(x)-f(y)|<\epsilon$ e $f(x) \sim f(y)$ pois $\epsilon$ é arbitrário. Reciprocamente, se ${ }^{*} f(x) \sim{ }^{*} f(y)$ para quaisquer $x, y \in \overline{x_{0}} \cap{ }^{*} S$ menores que $x_{0}$, e se $\epsilon$ for um número real positivo, então a condição fechada

$$
\left(\exists z \in{ }^{*} S\right)\left(\forall x, y \in{ }^{*} S\right)\left(\left.\left(z<x<x_{0} \wedge z<y<x_{0}\right) \Rightarrow\right|^{*} f(x)-{ }^{*} f(y) \mid<\epsilon\right)
$$

será verdadeira (pois bastaria escolher um $z \in{ }^{*} S$ com $z \sim x_{0}$ e $z<x_{0}$, o qual existe pois assumimos que $x_{0}$ é um ponto de acumulação de $S$ pela esquerda), e o resultado seguirá ao aplicarmos o PT a essa * $\mathbb{R}$-condição.

(h) Suponha que $l$ é um ponto de acumulação de $f$ nas proximidades de $x_{0}$ em $S$ pela esquerda. Isso significa que a condição fechada

$$
(\forall \epsilon \in \mathbb{R})(\forall x \in S)\left(\left(0<\epsilon \wedge x<x_{0}\right) \Rightarrow(\exists y \in S)\left(x<y<x_{0} \wedge|f(y)-l|<\epsilon\right)\right)
$$

é verdadeira, e, pelo PT, se $\epsilon$ for um número hiper-real positivo e infinitesimal, e se $x \in{ }^{*} S \operatorname{com} x \sim x_{0}$ e $x<x_{0}$, então existirá um $y \in{ }^{*} S \operatorname{com} x<y<x_{0}$ e ${ }^{*} f(y)-l \mid<\epsilon$, resultando em $y \sim x_{0} \mathrm{e}^{*} f(y) \sim l$. Reciprocamente, se ${ }^{*} f(x) \sim l$ para algum $x \in \overline{x_{0}} \cap{ }^{*} S$ menor que $x_{0}$, se $\epsilon$ for um número real positivo, e se $y$ for um elemento de $S$ menor que $x_{0}$, então teremos $y<x,\left.\right|^{*} f(x)-l \mid<\epsilon$, e a condição fechada

$$
\left(\exists x \in{ }^{*} S\right)\left(y<\left.x \wedge\right|^{*} f(x)-l \mid<\epsilon\right)
$$

será verdadeira. Nesse caso, pelo PT, existirá um $x \in S \operatorname{com} y<x$ e $|f(x)-l|<\epsilon$, resultando que $l$ será um ponto de acumulação de $f$ nas proximidades de $x_{0}$ em $S$ pela esquerda.

(i) Se $f$ for limitada superiormente nas proximidades de $x_{0}$ em $S$ pela esquerda, se $M$ for um número real tal que existe um número real positivo $\delta$ tal que a condição fechada

$$
(\forall x \in S)\left(0<x_{0}-x<\delta \Rightarrow f(x)<M\right)
$$

é verdadeira, e se $x \in \overline{x_{0}} \cap{ }^{*} S \operatorname{com} x<x_{0}$, então teremos $0<x_{0}-x<\delta$, e, pelo PT, teremos * $f(x)<M$. Se $M$ for um número real, e se tivermos ${ }^{*} f(x)<M$ para todo $x \in \overline{x_{0}} \cap{ }^{*} S$ menor que $x_{0}$, então a condição fechada

$$
\left(\exists \delta \in{ }^{*} \mathbb{R}\right)\left(0<\delta \wedge\left(\forall x \in{ }^{*} S\right)\left(0<x_{0}-x<\delta \Rightarrow{ }^{*} f(x)<M\right)\right)
$$

será verdadeira (pois bastaria escolher um $\delta$ infinitesimal e positivo), e o resultado seguirá ao aplicarmos o PT a essa * $\mathbb{R}$-condição.

(j) Se $f$ tiver um máximo local nas proximidades de $x_{0}$ em $S$ pela esquerda, se $\delta$ for um número real positivo tal que a condição fechada

$$
(\forall x \in S)\left(0<x_{0}-x<\delta \Rightarrow f(x) \leqslant f\left(x_{0}\right)\right)
$$


é verdadeira, e se $x \in \overline{x_{0}} \cap{ }^{*} S \operatorname{com} x<x_{0}$, então teremos $0<x_{0}-x<\delta$, e, pelo $\mathrm{PT}$, teremos ${ }^{*} f(x) \leqslant f\left(x_{0}\right)$. Se ${ }^{*} f(x) \leqslant f\left(x_{0}\right)$ para todo $x \in \overline{x_{0}} \cap{ }^{*} S$ menor que $x_{0}$, então a condição fechada

$$
\left(\exists \delta \in{ }^{*} \mathbb{R}\right)\left(0<\delta \wedge\left(\forall x \in{ }^{*} S\right)\left(0<x_{0}-x<\delta \Rightarrow{ }^{*} f(x) \leqslant f\left(x_{0}\right)\right)\right)
$$

será verdadeira (pois bastaria escolher um $\delta$ infinitesimal e positivo), e o resultado seguirá ao aplicarmos o PT a essa * R-condição.

As letras $(k)$ e $(l)$ são consequências imediatas da letra $(f)$.

Exemplo 2.42. Seja $X$ um subconjunto de $\mathbb{R}$. Empregando os mesmos procedimentos aplicados na demonstração do Teorema 2.41, prova-se que existirá um número real positivo $h$ com $|x-y| \geqslant h$ para quaisquer $x, y \in X$ distintos se, e somente se, para quaisquer $x, y \in{ }^{*} X \operatorname{com} x \sim y$ tivermos $x=y$. Esse será o caso para os exemplos $X=\mathbb{N}$ e $X=\mathbb{Z}$, e, para qualquer $X \subset \mathbb{R}$ desse tipo, teremos que toda função real definida em $X$ será uniformemente contínua em $X$ (Teorema 2.41, Letra $(e)$ ).

Exemplo 2.43. Seja $Y$ um subconjunto ilimitado superiormente de $\mathbb{R}$ tal que existe um número hiper-real positivo e infinito $N$ com $N \in{ }^{*} Y$ e $N+N^{-1} \in{ }^{*} Y$. Esse será o caso para $Y=\mathbb{Q}, Y=\operatorname{Irr}$ e $Y=\mathbb{R}$. Seja $n$ um número natural maior que 1 , e seja $p: Y \rightarrow \mathbb{R}$ a função dada por $p(x):=x^{n}$. Pelo PT, nota-se que ${ }^{*} p(x)=x^{n}\left(\forall x \in{ }^{*} Y\right)$, e

$$
\begin{aligned}
{ }^{*} p\left(N+N^{-1}\right) & =\left(N+N^{-1}\right)^{n} \\
& =\sum_{k=0}^{n}\left(\begin{array}{l}
n \\
k
\end{array}\right) N^{k}\left(N^{-1}\right)^{(n-k)} \\
& =\sum_{k=0}^{n}\left(\begin{array}{l}
n \\
k
\end{array}\right) N^{2 k-n} \\
& =h+H+{ }^{*} p(N)
\end{aligned}
$$

onde

$$
h:=\sum_{0 \leqslant k<n / 2}\left(\begin{array}{l}
n \\
k
\end{array}\right) N^{2 k-n}= \begin{cases}N^{-n}+n N^{2-n}+\cdots+\left(\begin{array}{c}
n \\
(n-2) / 2
\end{array}\right) N^{-2} & \text { se } n \text { for par } \\
N^{-n}+n N^{2-n}+\cdots+\left(\begin{array}{c}
n \\
(n-1) / 2
\end{array}\right) N^{-1} & \text { se } n \text { for ímpar }\end{cases}
$$

$\mathrm{e}$

$$
H:=\sum_{n / 2 \leqslant k<n}\left(\begin{array}{l}
n \\
k
\end{array}\right) N^{2 k-n}= \begin{cases}\left(\begin{array}{c}
n \\
n / 2
\end{array}\right)+\left(\begin{array}{c}
n \\
(n+2) / 2
\end{array}\right) N^{2}+\cdots+\left(\begin{array}{c}
n \\
n-1
\end{array}\right) N^{n-2} & \text { se } n \text { for par } \\
\left(\begin{array}{c}
n \\
(n+1) / 2
\end{array}\right) N+\left(\begin{array}{c}
n \\
(n+3) / 2
\end{array}\right) N^{3}+\cdots+\left(\begin{array}{c}
n \\
n-1
\end{array}\right) N^{n-2} & \text { se } n \text { for ímpar. }\end{cases}
$$

Nesse caso, como $h$ é infinitesimal e $H$ não é, teremos ${ }^{*} p\left(N+N^{-1}\right) \chi^{*} p(N)$, resultando que $p$ não é uniformemente contínua (Teorema 2.41, Letra $(e)$ ). 
Se $Z$ for um subconjunto limitado de $\mathbb{R}$, e se $u$ e $v$ forem elementos de ${ }^{*} Z$ com $u \sim v$, então $u$ e $v$ serão números hiper-reais finitos (Teorema 2.41, Letra $(a)$ ), e teremos $u^{n}-v^{n}=(u-v) \cdot k$, onde

$$
k:=u^{n-1}+u^{n-2} v^{1}+\cdots+u^{1} v^{n-2}+v^{n-1} .
$$

Nesse caso, como $u-v$ é infinitesimal e $k$ é finito (Teorema 1.18), teremos $u^{n} \sim v^{n}$, provando que a função polinomial $x \mapsto x^{n}$ é uniformemente contínua em $Z$.

Nas letras $(c)$ e $(d)$ do Teorema 2.41, note que $*\langle S\rangle \subset{ }^{*} S$ (Teorema 2.3, Letra $(e)$ ), resultando que as famílias de funções reais que convergem uniformemente para uma função real também convergem pontualmente para tal função.

Exemplo 2.44. Seja $\left\{f_{\lambda}\right\}_{\lambda \in(0, \infty)_{\mathbb{R}}}$ a família de funções $f_{\lambda}:(0,1)_{\mathbb{R}} \rightarrow \mathbb{R}$ dadas por $f_{\lambda}(x):=e^{-\lambda x}$. Mostraremos que $\lim _{\lambda \rightarrow \infty} f_{\lambda}=0$ pontualmente mas não uniformemente. Seja $\lambda$ um índice positivo e infinito, e seja $x$ um número real em $(0,1)_{\mathbb{R}}=*\left\langle(0,1)_{\mathbb{R}}\right\rangle$. Como $x$ é apreciável, o produto $\lambda x$ é infinito (Teorema 1.18), resultando em $e^{-\lambda x} \sim 0$ visto que $\lim _{y \rightarrow \infty} e^{-y}=0$, e provando que $\lim _{\lambda \rightarrow \infty} f_{\lambda}=0$ pontualmente (Teorema 2.41, Letra $(c)$ ). Além disso, temos

$$
0 \sim \lambda^{-1} \in(0,1)_{* \mathbb{R}}={ }^{*}\left((0,1)_{\mathbb{R}}\right)
$$

e

$$
e^{-\lambda \lambda^{-1}}=e^{-1} \neq 0
$$

provando que a família $\left\{f_{\lambda}\right\}_{\lambda \in(0, \infty)}$ não converge uniformemente para 0 (Teorema 2.41, Letra $(d))$.

Exemplo 2.45. Seja $\left\{g_{\lambda}\right\}_{\lambda \in(0, \infty)_{\mathbb{R}}}$ a família de funções $g_{\lambda}:[0,1]_{\mathbb{R}} \rightarrow \mathbb{R}$ dadas por $g_{\lambda}(x):=x e^{-\lambda x}$. Mostraremos que $\lim _{\lambda \rightarrow \infty} g_{\lambda}=0$ uniformemente. Seja $\lambda$ um índice positivo e infinito, e seja $x$ um número hiperreal em ${ }^{*}\left([0,1]_{\mathbb{R}}\right)=[0,1]_{* \mathbb{R}}$. Como $0<e^{-r} \leqslant 1\left(\forall r \in[0, \infty)_{\mathbb{R}}\right)$, pelo PT temos $0<e^{-r} \leqslant 1\left(\forall r \in[0, \infty)_{* \mathbb{R}}\right)$, e, como $0 \leqslant \lambda x$, temos que $e^{-\lambda x}$ é finito, implicando em $x e^{-\lambda x} \sim 0$ se $x$ for infinitesimal (Teorema 1.18). Se $x$ não for infinitesimal, então $\lambda x$ será infinito e teremos $e^{-\lambda x} \sim 0$, resultando em $x e^{-\lambda x} \sim 0$ pois $x$ é finito. Portanto, temos $x e^{-\lambda x} \sim 0$ em todo caso, e $\lim _{\lambda \rightarrow \infty} g_{\lambda}=0$ uniformemente (Teorema 2.41, Letra $(d)$ ).

Seja $I$ um conjunto de números reais, seja $i_{0}$ um ponto de acumulação de $I$ pela esquerda, seja $S$ um conjunto relacionado a $\mathbb{R}$, e seja $\left\{f_{i}\right\}_{i \in I}$ uma família de funções reais definidas em $S$ com índices em $I$. Pela Notação 2.40, se $i \in{ }^{*} I$, então a notação $f_{i}$ representa a função ${ }^{*} g(i)$, onde $g$ é a função com domínio $I$ definida por $g(i):=f_{i}$. Como cada função $f_{i}$ com $i \in I$ é definida em $S$, a condição fechada

$$
(\forall i \in I)(\forall r \in S)(\exists s \in \mathbb{R})(r, s) \in g(i)
$$


será verdadeira, resultando pelo PT que cada função $f_{i} \operatorname{com} i \in{ }^{*} I$ é definida em ${ }^{*} S$. Para cada $r \in S$, seja $g_{r}$ a função do tipo $I \rightarrow \mathbb{R}$ definida por $g_{r}(i):=f_{i}(r)$. Assim, a condição fechada

$$
(\forall i \in I)\left(g_{r}(i)=g(i)(r)\right)
$$

é verdadeira, e, pelo PT, temos ${ }^{*} g_{r}(i)=f_{i}\left({ }^{*} r\right)\left(\forall i \in{ }^{*} I\right)$.

Teorema 2.46 (Equivalências para Convergências de Famílias de Funções). Seja I um conjunto de números reais, seja $i_{0}$ um ponto de acumulação de I pela esquerda, seja $S$ um conjunto relacionado a $\mathbb{R}$, e seja $\left\{f_{i}\right\}_{i \in I}$ uma família de funções reais definidas em $S$ com indices em I. Temos:

(a) A familia $\left\{f_{i}\right\}_{i \in I}$ convergirá pontualmente em $S$ quando $i \rightarrow i_{0}$ pela esquerda se, $e$ somente se, $f_{i}(x) \sim f_{j}(x)$ para quaisquer $i, j \in \overline{i_{0}} \cap^{*} I$ menores que $i_{0} e$ para todo $x \in *\langle S\rangle$;

(b) A família $\left\{f_{i}\right\}_{i \in I}$ convergirá uniformemente em $S$ quando $i \rightarrow i_{0}$ pela esquerda se, e somente se, $f_{i}(x) \sim f_{j}(x)$ para quaisquer $i, j \in \overline{i_{0}} \cap{ }^{*} I$ menores que $i_{0} e$ para todo $x \in{ }^{*} S$.

Demonstração. As provas das condições necessárias dessas duas equivalências são consequências imediatas da transitividade da relação de proximidade infinita e das letras $(c)$ e $(d)$ do Teorema 2.41. Considere a função $g$ e as funções $g_{r}$ definidas no parágrafo imediatamente anterior a este teorema.

(a) Suponha que $f_{i}(x) \sim f_{j}(x)$ para quaisquer $i, j \in \overline{i_{0}} \cap^{*} I$ menores que $i_{0}$ e para todo $x \in *\langle S\rangle$. Assim, se $r$ for um elemento de $S$, então ${ }^{*} r \in *\langle S\rangle$, e teremos

$$
{ }^{*} g_{r}(i)=f_{i}\left({ }^{*} r\right) \sim f_{j}\left({ }^{*} r\right)={ }^{*} g_{r}(j)
$$

para quaisquer $i, j \in \overline{i_{0}} \cap^{*} I$ menores que $i_{0}$, implicando, pela letra (a) do Teorema 2.48, que o limite

$$
\lim _{i \rightarrow i_{0}^{-}} g_{r}(i)=\lim _{i \rightarrow i_{0}^{-}} f_{i}(r)
$$

existe. Nota-se que a família $\left\{f_{i}\right\}_{i \in I}$ converge pontualmente em $S$ para a função $f: S \rightarrow \mathbb{R}$ definida por $f(r):=\lim _{i \rightarrow i_{0}^{-}} f_{i}(r)$ quando $i \rightarrow i_{0}$ pela esquerda.

(b) Suponha que $f_{i}(x) \sim f_{j}(x)$ para quaisquer $i, j \in \overline{i_{0}} \cap^{*} I$ menores que $i_{0}$ e para todo $x \in{ }^{*} S$, seja $f$ a função definida na prova da letra $(a)$, e seja $\epsilon$ um número real positivo. Pela letra $(a)$, temos que $\lim _{i \rightarrow i_{0}^{-}} f_{i}=f$ pontualmente em $S$, e a condição fechada

$$
(\forall x \in S)(\exists \delta \in \mathbb{R})\left(0<\delta \wedge(\forall i \in I)\left(0<i_{0}-i<\delta \Rightarrow|g(i)(x)-f(x)|<\epsilon\right)\right)
$$


é verdadeira. Seja $x$ um elemento de ${ }^{*} S$. Portanto, pelo PT, existe um número hiper-real positivo $\delta$ tal que, para cada $i \in{ }^{*} I$ com $0<i_{0}-i<\delta$, temos $\left|f_{i}(x)-{ }^{*} f(x)\right|<\epsilon$, e podemos assumir ${ }^{6}$ que $\delta$ é infinitesimal. Seja ${ }^{7} j$ um elemento de ${ }^{*} I$ com $0<i_{0}-j<\delta$. Assim, temos $j \in \overline{i_{0}} \cap{ }^{*} I, j<i_{0},\left|f_{j}(x)-{ }^{*} f(x)\right|<\epsilon$, e se $i$ for um elemento de $\overline{i_{0}} \cap{ }^{*} I$ menor que $i_{0}$, então $f_{i}(x) \sim f_{j}(x)$ pela suposição, e $\left|f_{i}(x)-{ }^{*} f(x)\right|<\epsilon$. Nesse caso, como $\epsilon$ é arbitrário, teremos $f_{i}(x) \sim{ }^{*} f(x)$, provando que $\lim _{i \rightarrow i_{0}^{-}} f_{i}=f$ uniformemente em $S$ (Teorema 2.41, Letra $(d)$ ).

Voltando a atenção para as situações:

- $I=\left[n_{0}, \infty\right)_{\mathbb{Z}}$ e $i_{0}=\infty$ nas letras $(c)$ e $(d)$ do Teorema 2.41 e nas letras $(a)$ e $(b)$ do Teorema 2.46;

- $S=\left[n_{0}, \infty\right)_{\mathbb{Z}}$ e $x_{0}=\infty$ nas letras $(f),(g),(h)$ e $(i)$ do Teorema 2.41,

temos o seguinte corolário:

Corolário 2.47. Seja $S$ um conjunto relacionado a $\mathbb{R}$, seja $n_{0}$ um número inteiro, seja $\left\{f_{n}\right\}_{n \geqslant n_{0}}$ uma sequência de funções reais definidas em $S$, e seja $f$ uma função real definida em S. Teremos:

(a) $\lim _{n \rightarrow \infty} f_{n}=f$ pontualmente em $S$ se, e somente se, $f_{n}(x) \sim{ }^{*} f(x)$ para todo $n \in{ }^{*} \mathbb{N}_{\infty}$ e para todo $x \in *\langle S\rangle$;

(b) $\lim _{n \rightarrow \infty} f_{n}=f$ uniformemente em $S$ se, e somente se, $f_{n}(x) \sim{ }^{*} f(x)$ para todo $n \in{ }^{*} \mathbb{N}_{\infty}$ e para todo $x \in{ }^{*} S$;

(c) A sequência $\left\{f_{n}\right\}_{n \geqslant n_{0}}$ convergirá pontualmente em $S$ quando $n \rightarrow \infty$ se, e somente se, $f_{m}(x) \sim f_{n}(x)$ para quaisquer $m, n \in{ }^{*} \mathbb{N}_{\infty}$ e para todo $x \in *\langle S\rangle$;

(d) A sequência $\left\{f_{n}\right\}_{n \geqslant n_{0}}$ convergirá uniformemente em $S$ quando $n \rightarrow \infty$ se, e somente se, $f_{m}(x) \sim f_{n}(x)$ para quaisquer $m, n \in{ }^{*} \mathbb{N}_{\infty}$ e para todo $x \in{ }^{*} S$.

Seja $\left\{x_{n}\right\}_{n \geqslant n_{0}}$ uma sequência de números reais e seja l um número real estendido.

(e) $\quad \lim _{n \rightarrow \infty} x_{n}=l$ se, e somente se, $x_{n} \sim l$ para todo $n \in{ }^{*} \mathbb{N}_{\infty}$;

6 Se $\delta$ não for infinitesimal, então qualquer número hiper-real positivo e infinitesimal pode substituí-lo na demonstração.

7 Por hipótese, $i_{0}$ é um ponto de acumulação de $I$ pela esquerda, ou seja, a sentença fechada

$$
(\forall \delta \in \mathbb{R})\left(0<\delta \Rightarrow(\exists i \in I) 0<i_{0}-i<\delta\right)
$$

é verdadeira. A existência do elemento $j$ de ${ }^{*} I$ com $0<i_{0}-j<\delta$ segue pelo PT. 
(f) $\left\{x_{n}\right\}_{n \geqslant n_{0}}$ será uma sequência de Cauchy se, e somente se, $x_{m} \sim x_{n}$ para quaisquer $m, n \in{ }^{*} \mathbb{N}_{\infty}$;

(g) l será um ponto de acumulação de $\left\{x_{n}\right\}_{n \geqslant n_{0}}$ se, e somente se, $x_{n} \sim l$ para algum $n \in{ }^{*} \mathbb{N}_{\infty}$;

(h) $\left\{x_{n}\right\}_{n \geqslant n_{0}}$ será limitada superiormente se, e somente se, existir um número real $M$ tal que $x_{n}<M$ para todo $n \in{ }^{*} \mathbb{N}_{\infty}$.

No restante desta seção, empregaremos as novas perspectivas apresentadas no Teorema 2.41, no Teorema 2.46 e no Corolário 2.47 para demonstrar alguns teoremas importantes da Análise Real. O cunho dessas demonstrações se aproxima do estilo dos matemáticos dos séculos XVII e XVIII, destoando da tendência promovida por matemáticos proeminentes do século XIV como Bolzano, Weierstrass e Cantor, os quais desaprovavam veementemente o uso das quantidades infinitesimais e infinitas na Matemática.

Teorema 2.48. Seja $S$ um conjunto de números reais com um ponto de acumulação $x_{0}$ pela esquerda e seja $f$ uma função real definida em $S$. Temos:

(a) O limite $\lim _{\substack{x \rightarrow x_{0}^{-} \\ x \in S}} f(x)$ existirá se, e somente se, a função $f$ for uma função de Cauchy nas proximidades de $x_{0}$ em $S$ pela esquerda;

(b) (Teorema de Cauchy) O corpo ordenado dos números reais é Cauchy-completo. Ou seja, uma sequência $\left\{r_{n}\right\}_{n \geqslant n_{0}}$ de números reais será convergente se, e somente se, ela for uma sequência de Cauchy;

(c) (Teorema de Bolzano-Weierstrass $)^{8}$ Se $f$ for limitada nas proximidades de $x_{0}$ em $S$ pela esquerda, então $f$ terá um ponto de acumulação real nas proximidades de $x_{0}$ em $S$ pela esquerda.

Demonstração. Claramente, a letra (b) é uma consequência da letra (a).

(a) A condição necessária segue pela transitividade da relação de proximidade infinita. Se $f$ for uma função de Cauchy nas proximidades de $x_{0}$ em $S$ pela esquerda, então $f$ será limitada $^{9}$ nas proximidades de $x_{0}$ em $S$ pela esquerda, e se além disso $y$ for

8 Esta é uma generalização do Teorema de Bolzano-Weierstrass. A versão tradicional corresponde ao caso em que $x_{0}=+\infty$ e $S=\mathbb{N}$.

9 Existirá um $z \in S$ menor que $x_{0}$ tal que $|f(x)-f(y)|<1$ para quaisquer $x, y \in S$ entre $z$ e $x_{0}$. Assim, fixando um $y$ em $S$ entre $z$ e $x_{0}$, teremos

$$
f(y)-1<f(x)<f(y)+1\left(\forall x \in\left(z, x_{0}\right)_{S}\right),
$$

implicando que a função $f$ é limitada nas proximidades de $x_{0}$ em $S$ pela esquerda. 
um elemento de $\overline{x_{0}} \cap * S$ que é menor que $x_{0}$, então * $f(y)$ será finito (Teorema 2.41, Letra $(i))$, resultando em

$$
{ }^{*} f(z) \sim{ }^{*} f(y) \sim \operatorname{st}\left({ }^{*} f(y)\right)
$$

para todo $z \in \overline{x_{0}} \cap * S$ menor que $x_{0}$, provando que $\lim _{\substack{x \rightarrow x_{0}^{-} \\ x \in S}} f(x)=\operatorname{st}\left({ }^{*} f(y)\right)$.

(c) Como $x_{0}$ é um ponto de acumulação de $S$ pela esquerda, existe um $x \in{ }^{*} S$ menor que $x_{0}$ tal que $x \sim x_{0}$ (Teorema 2.41, Letra $(b)$ ), e, como $f$ é limitada nas proximidades de $x_{0}$ em $S$ pela esquerda, existe um número real positivo $M$ tal que $\left.\right|^{*} f(y) \mid<M$ para todo $y \in \overline{x_{0}} \cap{ }^{*} S$ menor que $x_{0}$ (Teorema 2.41, Letra $(i)$ ). Em particular, temos $\left|{ }^{*} f(x)\right|<M$, implicando que ${ }^{*} f(x)$ é finito. Assim, o número real st $\left({ }^{*} f(x)\right)$ é um ponto de acumulação real de $f$ nas proximidades de $x_{0}$ em $S$ pela esquerda (Teorema 2.41, Letra $(h)$ ).

Teorema 2.49. Seja $f$ uma função real definida no intervalo compacto $[a, b]_{\mathbb{R}}$, onde $a, b \in \mathbb{R}$ com $a<b$. Se $f$ for contínua em $[a, b]_{\mathbb{R}}$, então $f$ será uniformemente contínua em $[a, b]_{\mathbb{R}}$.

Demonstração. Sejam $x$ e $y$ dois elementos do conjunto $*\left([a, b]_{\mathbb{R}}\right)=[a, b]_{* \mathbb{R}}$ (Exemplo 2.10) tais que $x \sim y$. Assim, $x$ e $y$ são finitos. Seja $r:=\operatorname{st}(x)=\operatorname{st}(y)$. Como a função parte standard associada a ${ }^{*} \mathbb{R}$ é um morfismo entre domínios não estritamente ordenados, temos

$$
\text { st }(a) \leqslant \operatorname{st}(x) \leqslant \operatorname{st}(b),
$$

ou seja, temos $a \leqslant r \leqslant b$. Portanto, $f$ é contínua em $r$, e, como $x \sim r$ e $y \sim r$, temos

$$
{ }^{*} f(x) \sim f(r) \sim{ }^{*} f(y)
$$

provando que $f$ é uniformemente contínua em $[a, b]_{\mathbb{R}}$ (Teorema 2.41, Letra $(e)$ ).

Teorema 2.50 (Teorema do Ponto Crítico). Seja $f:(a, b)_{\mathbb{R}} \rightarrow \mathbb{R}$ uma função diferenciável em $c \in(a, b)_{\mathbb{R}}$. Se $f$ tiver um máximo local em c, então $f^{\prime}(c)=0$.

Demonstração. Seja $h$ um número hiper-real infinitesimal e positivo. Nota-se facilmente que $c \pm h \in(a, b)_{* \mathbb{R}}$, e temos ${ }^{*} f(c \pm h)-f(c) \leqslant 0$ (Teorema 2.41, Letra $(j)$ ), resultando em

$$
f^{\prime}(c) \sim \frac{{ }^{*} f(c+h)-f(c)}{h} \leqslant 0 \leqslant \frac{{ }^{*} f(c-h)-f(c)}{-h} \sim f^{\prime}(c)
$$

pela letra $(l)$ do Teorema 2.41. Assim, temos $f^{\prime}(c) \sim 0$ e $f^{\prime}(c)=0$ (Proposição 1.79). 
Teorema 2.51 (Teorema da Função Inversa). Seja $f:(a, b)_{\mathbb{R}} \rightarrow \mathbb{R}$ contínua, estritamente monotônica e diferenciável em $x \in(a, b)_{\mathbb{R}}$ com $f^{\prime}(x) \neq 0$. A função inversa $g:=f^{-1}$ é diferenciável em $y:=f(x)$, e

$$
g^{\prime}(y)=\frac{1}{f^{\prime}(x)}
$$

Demonstração. Pelo Teorema do Valor Intermediário e pela monotonicidade de $f$, nota-se que a função $g$ é definida em um intervalo aberto em $\mathbb{R}$ contendo $y$. Assim, fixando um número hiper-real infinitesimal $h$, temos que a função ${ }^{*} g$ é definida em $y+h$. Note que as funções ${ }^{*} f \mathrm{e}^{*} g$ são inversas (Teorema 2.14, Letra $(e)$ ). Provaremos que

$$
\frac{{ }^{*} g(y+h)-g(y)}{h}=\frac{{ }^{*} g(f(x)+h)-x}{h} \sim \frac{1}{f^{\prime}(x)} .
$$

Os número ${ }^{*} g(f(x)+h)$ é finito, uma vez que ele pertence a ${ }^{*}\left((a, b)_{\mathbb{R}}\right)=(a, b)_{* \mathbb{R}}$. Se ${ }^{*} g(f(x)+h) \not x$, então $\overline{{ }^{*} g(f(x)+h)} \neq \bar{x}$, e existirá um número real $r$ (estritamente) entre ${ }^{*} g(f(x)+h)$ e $x$ (Proposição 1.44). Nesse caso, como $f$ é estritamente monotônica, ${ }^{*} f$ será estritamente monotônica pelo PT, e $f(r)$ estará entre $f(x)+h$ e $f(x)$, implicando em $f(r) \sim f(x), f(r)=f(x)$, e $r=x$, o que é absurdo pela maneira que obtivemos $r$. Portanto, temos ${ }^{*} g(f(x)+h) \sim x$. Seja $Q$ o número hiper-real dado por

$$
\begin{aligned}
Q & :=\frac{{ }^{*} f\left({ }^{*} g(f(x)+h)\right)-f(x)}{{ }^{*} g(f(x)+h)-x} \\
& =\frac{h}{{ }^{*} g(f(x)+h)-x} \\
& =\left(\frac{{ }^{*} g(y+h)-g(y)}{h}\right)^{-1} .
\end{aligned}
$$

Como $f$ é diferenciável em $x$, temos $Q \sim f^{\prime}(x) \neq 0, Q$ é apreciável, e a diferença

$$
\frac{1}{Q}-\frac{1}{f^{\prime}(x)}=\frac{f^{\prime}(x)-Q}{Q \cdot f^{\prime}(x)}
$$

é infinitesimal (Teorema 1.18).

Após ter contemplado as demonstrações dos últimos quatro teoremas, o leitor pode ter notado que tais argumentos são consideravelmente mais simples e diretos que as provas apresentadas nos cursos tradicionais de Cálculo e Análise Real. Uma possível interpretação para tal simplificação é o fortalecimento do sistema de axiomas utilizado na argumentação manifestada nesta seção. Nos Capítulos 3 e 5 veremos que o Axioma da Escolha é imprescindível na construção do monomorfismo não standard $*$, enquanto as provas tradicionais dos teoremas basilares da Análise utilizam apenas uma versão mais fraca daquele axioma conhecida como Axioma da Escolha Contável (40). De modo genérico, a Análise Não Standard pode ser vista como uma maneira criativa e eficiente de aproveitar melhor o potencial do Axioma da Escolha, mesmo em parcelas da Matemática onde apenas versões mais fracas desse axioma são estritamente necessárias. 


\subsection{Somas Hiperfinitas}

Seja $\mathcal{S}: \operatorname{Int}(\mathbb{Z},<) \mathbb{R} \rightarrow \mathbb{R}$ a função que associa uma sequência finita de números reais à sua soma, ou seja, a função definida recursivamente sobre o comprimento das sequências finitas em $\operatorname{Int}(\mathbb{Z},<) \mathbb{R}$ como segue:

$(\mathcal{S} 1) \mathcal{S}\left(\left\{x_{i}\right\}_{m \leqslant i \leqslant m}\right):=x_{m}$ para qualquer sequência $\left\{x_{i}\right\}_{m \leqslant i \leqslant m}$ em ${ }^{\operatorname{Int}(\mathbb{Z},<)} \mathbb{R}$ cujo conjunto de índices é unitário;

$(\mathcal{S} 2) \mathcal{S}\left(\left\{x_{i}\right\}_{m \leqslant i \leqslant n}\right):=\mathcal{S}\left(\left\{x_{i}\right\}_{m \leqslant i \leqslant n-1}\right)+x_{n}$ para qualquer sequência finita de números reais $\left\{x_{i}\right\}_{m \leqslant i \leqslant n}$ com $m, n \in \mathbb{Z}$ e $m<n$.

Tais cláusulas definem os valores de $\mathcal{S}$ sobre cada sequência não nula pertencente a $\operatorname{Int}(\mathbb{Z},<) \mathbb{R}$, e, a fim de completar a definição de $\mathcal{S}$, definimos $\mathcal{S}(\emptyset):=0 .{ }^{10}$

Pelos Teoremas 2.14 e 2.31, temos que ${ }^{*} \mathcal{S}$ é uma função do tipo $\mathbb{I} \cap \operatorname{Int}\left({ }^{*} \mathbb{Z},<\right)\left({ }^{*} \mathbb{R}\right) \rightarrow{ }^{*} \mathbb{R}$, e, assim como fizemos na Notação 2.40 , dizemos que as famílias pertencentes ao conjunto ${ }^{\operatorname{Int}\left({ }^{*} \mathbb{Z},<\right)}\left({ }^{*} \mathbb{R}\right)$ são hipersequências. ${ }^{11}$

Definição 2.52. Seja $x_{m} x_{m+1} \ldots x_{n}$ uma hipersequência interna de números hiper-reais. O número hiper-real

$$
{ }^{*} \mathcal{S}\left(x_{m} x_{m+1} \ldots x_{n}\right)
$$

é chamado de soma hiperfinita da hipersequência interna $x_{m} x_{m+1} \ldots x_{n}$, e, por abuso de linguagem, ele é denotado por $\sum_{i=m}^{n} x_{i}$ ou por

$$
x_{m}+x_{m+1}+\cdots+x_{n}
$$

Veremos no teorema a seguir que as somas hiperfinitas compartilham algumas propriedades com as somas finitas.

$\overline{10}$ Como $0 \in \mathbb{R}$ é o elemento neutro da adição, a função $\mathcal{S}$ pode ser definida pelas seguintes condições:

$(\mathcal{S} 0) \quad \mathcal{S}(\emptyset):=0$

$\left(\mathcal{S} 2^{\prime}\right) \quad \mathcal{S}\left(\left\{x_{i}\right\}_{m \leqslant i \leqslant n}\right):=\mathcal{S}\left(\left\{x_{i}\right\}_{m \leqslant i \leqslant n-1}\right)+x_{n}$ para qualquer sequência finita de números reais $\left\{x_{i}\right\}_{m \leqslant i \leqslant n} \operatorname{com} m, n \in \mathbb{Z}$ e $m \leqslant n$.

Note que $\left\{x_{i}\right\}_{m \leqslant i \leqslant m-1}=\emptyset$ e

$$
\mathcal{S}\left(\left\{x_{i}\right\}_{m \leqslant i \leqslant m}\right)=\mathcal{S}(\emptyset)+x_{m}=0+x_{m}=x_{m} .
$$

11 Em geral, os matemáticos chamam de sequências apenas as famílias cujos conjuntos de índices são bem ordenados. 
Teorema 2.53 (Propriedades das Somas Hiperfinitas). Se $x_{m} \ldots x_{n} \ldots x_{p}$ e $y_{m} \ldots y_{n}$ forem duas hipersequências internas de números hiper-reais (onde $m \leqslant n<p$ são números hiperinteiros), se $r \in{ }^{*} \mathbb{R}$, e se $j \in *^{*} \mathbb{Z}$, então teremos:
(a) $\sum_{i=m}^{n}\left(x_{i}+r \cdot y_{i}\right)=\sum_{i=m}^{n} x_{i}+r \cdot \sum_{i=m}^{n} y_{i}$;
(Linearidade)
(b) $\sum_{i=m}^{n} x_{i}+\sum_{i=n+1}^{p} x_{i}=\sum_{i=m}^{p} x_{i}$;
(Aditividade)
(c) $\sum_{i=m}^{n} x_{i}=\sum_{i=m+j}^{n+j} x_{i-j}$;
(Invariância Translacional)
(d) $\sum_{i=m}^{n} x_{i} \leqslant \sum_{i=m}^{n} y_{i}$ se $x_{i} \leqslant y_{i}\left(\forall i \in[m, n]_{*_{\mathbb{Z}}}\right)$;
(Monotonicidade)
(e) $\left|\sum_{i=m}^{n} x_{i}\right| \leqslant \sum_{i=m}^{n}\left|x_{i}\right|$.
(Desigualdade Triangular)

Demonstração. Sejam D , Sup e Inf as funções definidas nos Exemplos 2.36, 2.37 e 2.38, sejam

$$
\begin{gathered}
\mathcal{E}:=\left\{(x, y) \in\left({ }^{\operatorname{Int}(\mathbb{Z},<)} \mathbb{R}\right)^{2}: \mathcal{D}(x)=\mathcal{D}(y)\right\}, \\
\leqq:=\{(x, y) \in \mathcal{E} \vdots(\forall S \in \mathcal{P}(\mathbb{Z}))(S=\mathcal{D}(x) \Rightarrow(\forall n \in S) x(n) \leqslant y(n))\},
\end{gathered}
$$

e

$$
\mathcal{C}:=\left\{(x, y) \in\left({ }^{\operatorname{Int}(\mathbb{Z},<)} \mathbb{R}\right)^{2}: \mathcal{D}(x) \neq \emptyset \neq \mathcal{D}(y) \wedge \operatorname{Sup}(\mathcal{D}(x))+1=\operatorname{lnf}(\mathcal{D}(y))\right\}
$$

subconjuntos do produto cartesiano $\left({ }^{\operatorname{Int}(\mathbb{Z},<)} \mathbb{R}\right)^{2}=\left({ }^{\operatorname{Int}(\mathbb{Z},<)} \mathbb{R}\right) \times(\operatorname{Int}(\mathbb{Z},<) \mathbb{R})$, e sejam

- $\oplus: \mathcal{E} \rightarrow{ }^{\operatorname{Int}(\mathbb{Z},<)} \mathbb{R}$ definida de modo que $\mathcal{D}(x \oplus y):=\mathcal{D}(x)=\mathcal{D}(y)$ e

$$
(x \oplus y)(n):=x(n)+y(n) ;
$$

- $\otimes: \mathbb{R} \times{ }^{\operatorname{Int}(\mathbb{Z},<)} \mathbb{R} \rightarrow^{\operatorname{Int}(\mathbb{Z},<)} \mathbb{R}$ definida de modo que $\mathcal{D}(r \otimes x):=\mathcal{D}(x) \mathrm{e}$

$$
(r \otimes x)(n):=r \cdot x(n) ;
$$

- $\perp: \mathcal{C} \rightarrow{ }^{\operatorname{Int}(\mathbb{Z},<)} \mathbb{R}$ definida de modo que $\mathcal{D}(x \perp y)=\mathcal{D}(x) \cup \mathcal{D}(y) \mathrm{e}$

$$
(x \perp y)(n):= \begin{cases}x(n) & \text { se } n \in \mathcal{D}(x) \\ y(n) & \text { se } n \in \mathcal{D}(y)\end{cases}
$$


- Trans $:{ }^{\operatorname{Int}(\mathbb{Z},<)} \mathbb{R} \rightarrow{ }^{\operatorname{Int}(\mathbb{Z},<)} \mathbb{R}$ definida de modo que $\mathcal{D}(\operatorname{Trans}(x)):=\mathcal{D}(x)+j$ e

$$
\operatorname{Trans}(x)(n):=x(n-j)
$$

- Abs : ${ }^{\operatorname{Int}(\mathbb{Z},<)} \mathbb{R} \rightarrow{ }^{\operatorname{Int}(\mathbb{Z},<)} \mathbb{R}$ definida de modo que $\mathcal{D}($ Abs $(x)):=\mathcal{D}(x)$ e

$$
\operatorname{Abs}(x)(n):=|x(n)| \text {. }
$$

Os resultados das letras $(a),(b),(c),(d)$ e $(e)$ seguem ao aplicarmos o PT às respectivas condições fechadas a seguir:

1. $(\forall x, y \in \operatorname{Int}(\mathbb{Z},<) \mathbb{R})(\forall r \in \mathbb{R})((x, y) \in \mathcal{E} \Rightarrow \mathcal{S}(x \oplus(r \otimes y))=\mathcal{S}(x)+r \cdot \mathcal{S}(y))$

2. $(\forall x, y \in \operatorname{Int}(\mathbb{Z},<) \mathbb{R})((x, y) \in \mathcal{C} \Rightarrow \mathcal{S}(x \perp y)=\mathcal{S}(x)+\mathcal{S}(y))$

3. $(\forall x \in \operatorname{Int}(\mathbb{Z},<) \mathbb{R}) \mathcal{S}(x)=\mathcal{S}(\operatorname{Trans}(x)) ;$

4. $(\forall x, y \in \operatorname{Int}(\mathbb{Z},<) \mathbb{R})(((x, y) \in \mathcal{E} \wedge x \leqq y) \Rightarrow \mathcal{S}(x) \leqslant \mathcal{S}(y))$

5. $(\forall x \in \operatorname{Int}(\mathbb{Z},<) \mathbb{R})|\mathcal{S}(x)| \leqslant \mathcal{S}(\operatorname{Abs}(x))$.

Deixaremos para o leitor a demonstração da propriedade $\sum_{i=n}^{n} x_{i}=x_{n}$.

Corolário 2.54. Se $x_{m} \ldots x_{n}$ for uma hipersequência interna de números hiper-reais (onde $m<n$ são números hiperinteiros), então teremos

$$
\sum_{i=m+1}^{n}\left(x_{i}-x_{i-1}\right)=x_{n}-x_{m}
$$

Demonstração. Temos

$$
\begin{aligned}
\sum_{i=m+1}^{n}\left(x_{i}-x_{i-1}\right) & =\sum_{i=m+1}^{n} x_{i}-\sum_{i=m+1}^{n} x_{i-1} \\
& =\left(\sum_{i=m+1}^{n-1} x_{i}+\sum_{i=n}^{n} x_{i}\right)-\left(\sum_{i=m+1}^{m+1} x_{i-1}+\sum_{i=m+2}^{n} x_{i-1}\right) \\
& =\left(\sum_{i=m+1}^{n-1} x_{i}+x_{n}\right)-\left(x_{(m+1)-1}+\sum_{i=(m+2)-1}^{n-1} x_{(i-1)+1}\right) \\
& =x_{n}-x_{m} .
\end{aligned}
$$


Pode-se definir produtos hiperfinitos de hipersequências de números hiper-reais de modo análogo ao que fizemos nesta seção para as somas, e, sistematicamente aplicando o PT, prova-se que tais produtos hiperfinitos herdam várias propriedades dos produtos finitos. Veremos no Capítulo 4 que é possível estender qualquer lei de composição associativa e interna em um conjunto interno para uma operação sobre hipersequências hiperfinitas de elementos desse conjunto.

\subsection{Atalho Dedutivo Usual}

Na literatura dedicada à Análise Não Standard, é comum que as respectivas condições 1-5 apresentadas na demonstração do Teorema 2.53 sejam expressas das seguintes maneiras equivalentes:

$$
\begin{array}{r}
1^{\prime} . \quad(\forall x, y \in \operatorname{Int}(\mathbb{Z},<) \mathbb{R})(\forall r \in \mathbb{R})(\operatorname{dom}(x)=\operatorname{dom}(y) \Rightarrow \mathcal{S}(x+(r \cdot y))=\mathcal{S}(x)+r \cdot \mathcal{S}(y)) ; \\
2^{\prime} . \quad(\forall x, y \in \operatorname{Int}(\mathbb{Z},<) \mathbb{R})((\operatorname{dom}(x) \neq \emptyset \neq \operatorname{dom}(y) \wedge \sup (\operatorname{dom}(x))+1=\inf (\operatorname{dom}(y))) \\
\Rightarrow \mathcal{S}(x y)=\mathcal{S}(x)+\mathcal{S}(y)) ; \\
\begin{array}{r}
(\forall x, y \in \operatorname{Int}(\mathbb{Z},<) \mathbb{R})(\forall S \in \mathcal{P}(\mathbb{Z}))((\operatorname{dom}(x)+j=S=\operatorname{dom}(y) \\
\left.\left.3^{\prime} . \quad(\forall i \in S) y(i)=x(i-j)\right) \Rightarrow \mathcal{S}(x)=\mathcal{S}(y)\right) ; \\
4^{\prime} . \quad(\forall x, y \in \operatorname{Int}(\mathbb{Z},<) \mathbb{R})(\forall S \in \mathcal{P}(\mathbb{Z}))((\operatorname{dom}(x)=S=\operatorname{dom}(y) \wedge(\forall i \in S) x(i) \leqslant y(i)) \\
\quad \Rightarrow \mathcal{S}(x) \leqslant \mathcal{S}(y)) ; \\
5^{\prime} . \quad(\forall x, y \in \operatorname{Int}(\mathbb{Z},<) \mathbb{R})(\forall S \in \mathcal{P}(\mathbb{Z}))((\operatorname{dom}(x)=S=\operatorname{dom}(y) \wedge(\forall i \in S) y(i)=|x(i)|) \\
\Rightarrow|\mathcal{S}(x)| \leqslant \mathcal{S}(y)) .
\end{array}
\end{array}
$$

A extensa série de definições prévias $\left(\mathcal{D}, \mathcal{P}_{\text {sup }}(\mathbb{R})\right.$, Sup, Inf, $\mathcal{E}, \leqq, \mathcal{C}, \oplus, \otimes, \perp$, Trans e Abs) gerada com o objetivo de revelar as condições 1-5 não foi necessária para que pudéssemos escrever as condições 1'-5', de modo que os objetos dessa série foram substituídos por relações mais comuns na prática da Matemática. Em contrapartida, não pode-se aplicar o PT a tais condições, visto que a presença dos operadores dom, sup e inf, os quais são operadores entre classes próprias e não são $\mathbb{R}$-objetos, viola um dos requisitos assentados na Seção 2.3.

Seja $\phi\left(x_{1} \ldots x_{m}, y_{1} \ldots y_{n}\right)$ uma $\mathbb{R}$-condição completamente aberta que segue às regras que haviam sido predeterminadas na Seção 2.3 , sejam $a_{1} \ldots a_{m} \mathbb{R}$-objetos, e considere que $A_{1} \ldots A_{n}$ são classes não relacionadas a $\mathbb{R}$ tais que existem $\mathbb{R}$-conjuntos $A_{1}^{\prime} \ldots A_{n}^{\prime}$ que observam a seguinte exigência:

(SubS) Cada $A_{i}^{\prime}\left(\right.$ resp. * $\left.A_{i}^{\prime}\right)$ é um "substituto satisfatório" para a classe $A_{i}$ no contexto dos $\mathbb{R}$-conjuntos (resp. ${ }^{*} \mathbb{R}$-conjuntos), de modo que a fórmula

$$
\phi\left(a_{1} \ldots a_{m}, A_{1} \ldots A_{n}\right) \quad\left(\operatorname{resp} . \phi\left({ }^{*} a_{1} \ldots{ }^{*} a_{m}, A_{1} \ldots A_{n}\right)\right)
$$


seja metamatematicamente equivalente à fórmula

$$
\phi\left(a_{1} \ldots a_{m}, A_{1}^{\prime} \ldots A_{n}^{\prime}\right) \quad\left(\operatorname{resp} . \phi\left({ }^{*} a_{1} \ldots{ }^{*} a_{m},{ }^{*} A_{1}^{\prime} \ldots{ }^{*} A_{n}^{\prime}\right)\right)
$$

Nesse caso, por abuso de linguagem, a condição fechada $\phi\left(a_{1} \ldots a_{m}, A_{1}^{\prime} \ldots A_{n}^{\prime}\right)$ é denotada por

$$
\phi\left(a_{1} \ldots a_{m}, A_{1} \ldots A_{n}\right),
$$

e a $*$-transformação dessa condição, dada por $\phi\left({ }^{*} a_{1} \ldots{ }^{*} a_{m},{ }^{*} A_{1}^{\prime} \ldots{ }^{*} A_{n}^{\prime}\right)$, é denotada por

$$
\phi\left({ }^{*} a_{1} \ldots{ }^{*} a_{m}, A_{1} \ldots A_{n}\right)
$$

O leitor é encorajado a se habituar a reconhecer rapidamente quando uma classe $A$ pode ser restrita a um $\mathbb{R}$-conjunto $A^{\prime}$ que satisfaz a (SubS), pois frequentemente tal conjunto $A^{\prime}$ não é mencionado explicitamente nas argumentações e demonstrações. O uso desse abuso de linguagem é indispensável na prática da Análise Não Standard, visto que ele abrevia consideravelmente o processo dedutivo vinculado à aplicação regular do Princípio da Transferência. No caso do Teorema 2.53, percebe-se que uma extensa série de definições prévias foram necessárias para que pudéssemos escrever as condições 1-5 seguindo às regras que haviam sido predeterminadas na Seção 2.3, e a maioria dos autores da área se poupariam dessa incumbência. Esse atalho dedutivo é dispensado nesta dissertação apenas até esta seção.

Exemplo 2.55. Em diversas situações, poderemos empregar a função dom, a qual é definida na classe das funções cujos domínios são conjuntos, em nossas condições matemáticas. Em particular, quando o contexto demandar uma função que compute o domínio das fun-

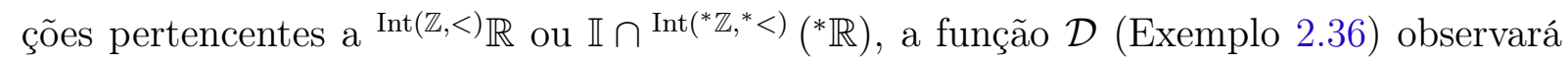
(SubS) para a função-classe dom.

Exemplo 2.56. Em diversas situações, poderemos empregar a função sup, a qual é definida na classe das ordens parciais que têm um supremo, em nossas condições matemáticas. Em particular, quando o contexto demandar uma função que compute o supremo dos elementos de $\mathcal{P}_{\text {sup }}(\mathbb{R})$ ou ${ }^{*} \mathcal{P}_{\text {sup }}(\mathbb{R})$ (Exemplo 2.37), a função Sup (Exemplo 2.38) observará (SubS) para a função-classe sup.

Exemplo 2.57. A relação $\subset$ pode ser restrita a qualquer conjunto de subconjuntos de uma classe. Seja $₫$ a relação

$$
\Subset:=\subset \cap(\mathcal{P}(\mathbb{R}) \times \mathcal{P}(\mathbb{R}))
$$

Note que $₫ \subset \mathcal{P}(\mathbb{R}) \times \mathcal{P}(\mathbb{R}) \mathrm{e}^{*} \Subset \subset\left(\mathbb{I} \cap \mathcal{P}\left({ }^{*} \mathbb{R}\right)\right) \times\left(\mathbb{I} \cap \mathcal{P}\left({ }^{*} \mathbb{R}\right)\right.$ ) (Corolário 2.5; Teoremas 2.12 e 2.31). Temos que a condição

$$
(\forall S, T \in \mathcal{P}(\mathbb{R}))(S \Subset T \Leftrightarrow(\forall x \in S) x \in T)
$$


é verdadeira, e, aplicando o PT a essa condição fechada, temos que a condição

$$
\left(\forall S, T \in \mathbb{I} \cap \mathcal{P}\left({ }^{*} \mathbb{R}\right)\right)\left(S^{*} \Subset T \Leftrightarrow(\forall x \in S) x \in T\right)
$$

é verdadeira, resultando que $\Subset \mathrm{e}^{*} \Subset$ são restrições de $\subset$. Assim, quando o contexto demandar uma relação binária de inclusão entre os elementos de $\mathcal{P}(\mathbb{R})$ ou $\mathbb{I} \cap \mathcal{P}\left({ }^{*} \mathbb{R}\right)$, a relação $\oplus$ observará $($ SubS) para a relação-classe $\subset$.

Exemplo 2.58. Seja Func a relação binária tal que $(f, B) \in$ Func se, e somente se, tivermos que $f$ é uma função com domínio $\mathbb{R}$ e contradomínio $B$, e seja $F$ unc a relação

$$
\text { Func }:=\text { Func } \cap\left({ }^{\mathbb{R}} \times \mathbb{P}(\mathbb{R})\right) \text {. }
$$

Note que Func $\subset \mathbb{R} \mathbb{R} \times \mathcal{P}(\mathbb{R})$ e

$$
\left.{ }^{*} \text { Func } \subset\left(\mathbb{I} \cap{ }^{*} \mathbb{R}\right)\left({ }^{*} \mathbb{R}\right)\right) \times\left(\mathbb{I} \cap \mathcal{P}\left({ }^{*} \mathbb{R}\right)\right)
$$

pelo Corolário 2.5 e pelos Teoremas 2.12 e 2.31. Claramente Func é uma restrição de Func. É possível (porém bastante maçante) obter uma $\mathbb{R}$-condição fechada que descreve a relação Func plenamente, e, aplicando o PT a tal condição, obtém-se que *Func é uma restrição de Func. Assim, quando o contexto demandar uma relação binária que associe uma função em ${ }^{\mathbb{R}} \mathbb{R}$ ou $\mathbb{I} \cap{ }^{(* \mathbb{R})}\left({ }^{*} \mathbb{R}\right)$ a um possível contradomínio em $\mathcal{P}(\mathbb{R})$ ou $\mathbb{I} \cap \mathcal{P}\left({ }^{*} \mathbb{R}\right)$ para tal função, a relação Func observará (SubS) para a relação-classe Func.

\subsection{Aplicações na Riemann-Integrabilidade}

Na história do Cálculo Integral, o primeiro aparecimento do símbolo $\int$, a letra S alongada, ocorreu no manuscrito Analyseos tetragonisticae pars secunda de Leibniz, datado de 29 de Outubro de 1675. Nele, o autor afirma que o uso da letra S para denotar integrais representa a palavra soma em Latim, summa, visto que ele concebia o processo de integração como uma soma infinita de somandos infinitesimais (35).

Veremos que a noção de Riemann-integrabilidade pode ser formalmente reescrita de uma maneira bastante semelhante à idealizada por Leibniz. De fato, sob a perspectiva da Análise Não Standard, uma integral de Riemann é a parte standard de uma soma hiperfinita de números hiper-reais infinitesimais.

Definição 2.59. Sejam $a$ e $b$ dois números reais com $a<b$, e seja $n \in{ }^{*} \mathbb{N}$. Um par de hipersequências de números hiper-reais

$$
P=\left(x_{0} x_{1} \ldots x_{n}, \pi_{1} \pi_{2} \ldots \pi_{n}\right)
$$


é dito ser uma hiperpartição (etiquetada) do intervalo $[a, b]_{* \mathbb{R}}$ se

$$
a=x_{0}<x_{1}<\cdots<x_{n}=b
$$

$\mathrm{e}$

$$
x_{i-1} \leqslant \pi_{i} \leqslant x_{i}\left(\forall i \in[1, n]_{* \mathbb{N}}\right) .
$$

Nesse caso, dizemos que $P$ é refinada se $x_{i}-x_{i-1} \sim 0\left(\forall i \in[1, n]_{* \mathbb{N}}\right)$.

Se $P$ for interna, então pelo Teorema 2.26 as hipersequências $x_{0} x_{1} \ldots x_{n}$ e $\pi_{1} \pi_{2} \ldots \pi_{n}$ serão internas, e o subconjunto

$$
S:=\left\{x_{i}-x_{i-1} \vdots 1 \leqslant i \leqslant n\right\}
$$

de ${ }^{*} \mathbb{R}$ será interno pelo PDI. Nesse caso, se $P$ for refinada, então $S$ terá uma cota superior em ${ }^{*} \mathbb{R}$ (e.g., qualquer número real positivo), resultando na existência do supremo

$$
\sup _{* \mathbb{R}}(S)=\sup _{1 \leqslant i \leqslant n}\left(x_{i}-x_{i-1}\right),
$$

o qual será infinitesimal.

Teorema 2.60. Seja $f:[a, b]_{\mathbb{R}} \rightarrow \mathbb{R}$ uma função limitada. Essa função será Riemannintegrável se, e somente se, existir um número real I tal que

$$
\sum_{i=1}^{n}{ }^{*} f\left(\pi_{i}\right) \cdot\left(x_{i}-x_{i-1}\right) \sim I
$$

para toda hiperpartição refinada e interna de $[a, b]_{* \mathbb{R}}$ dada por

$$
P=\left(x_{0} x_{1} \ldots x_{n}, \pi_{1} \pi_{2} \ldots \pi_{n}\right) \text {. }
$$

Nesse caso, teremos $\int f=I$.

Demonstração. Suponha que $f$ é Riemann-integrável. Seja $P=\left(x_{0} x_{1} \ldots x_{n}, \pi_{1} \pi_{2} \ldots \pi_{n}\right)$ uma hiperpartição interna e refinada de $[a, b]_{* \mathbb{R}}$, e seja $\epsilon>0$ um número real. Assim, existe um $\delta>0$ real tal que a condição fechada

$$
\begin{gathered}
\text { "| }\left|\sum_{i=1}^{m} f\left(\rho_{i}\right) \cdot\left(y_{i}-y_{i-1}\right)-\int f\right|<\epsilon \text { para toda partição } \\
Q=\left(y_{0} y_{1} \ldots y_{m}, \rho_{1} \rho_{2} \ldots \rho_{m}\right) \\
\operatorname{de}[a, b]_{\mathbb{R}} \operatorname{com} \sup _{1 \leqslant i \leqslant m}\left(y_{i}-y_{i-1}\right)<\delta "
\end{gathered}
$$

é verdadeira, e, aplicando o PT a tal condição, temos que a condição fechada

$$
\begin{gathered}
\text { "| }\left|\sum_{i=1}^{m}{ }^{*} f\left(\rho_{i}\right) \cdot\left(y_{i}-y_{i-1}\right)-\int f\right|<\epsilon \text { para toda hiperpartição interna } \\
Q=\left(y_{0} y_{1} \ldots y_{m}, \rho_{1} \rho_{2} \ldots \rho_{m}\right) \\
\text { de }[a, b]_{* \mathbb{R}} \operatorname{com} \sup _{1 \leqslant i \leqslant m}\left(y_{i}-y_{i-1}\right)<\delta "
\end{gathered}
$$


é verdadeira. Como $P$ é interna e refinada, temos que $\sup _{1 \leqslant i \leqslant n}\left(x_{i}-x_{i-1}\right)$ é infinitesimal e $\sup _{1 \leqslant i \leqslant n}\left(x_{i}-x_{i-1}\right)<\delta$, resultando em

$$
\left|\sum_{i=1}^{m}{ }^{*} f\left(\pi_{i}\right) \cdot\left(x_{i}-x_{i-1}\right)-\int f\right|<\epsilon
$$

e

$$
\sum_{i=1}^{m} * f\left(\pi_{i}\right) \cdot\left(x_{i}-x_{i-1}\right) \sim \int f
$$

pois $\epsilon$ é arbitrário. Portanto, a condição necessária do teorema está provada.

Suponha que $I$ é um número real tal que

$$
\sum_{i=1}^{n} * f\left(\pi_{i}\right) \cdot\left(x_{i}-x_{i-1}\right) \sim I
$$

para toda hiperpartição refinada e interna de $[a, b]_{* \mathbb{R}}$ dada por

$$
P=\left(x_{0} x_{1} \ldots x_{n}, \pi_{1} \pi_{2} \ldots \pi_{n}\right)
$$

Seja $\epsilon$ um número real positivo, e seja $\delta$ um número hiper-real positivo e infinitesimal. Como toda hiperpartição interna

$$
Q=\left(y_{0} y_{1} \ldots y_{m}, \rho_{1} \rho_{2} \ldots \rho_{m}\right)
$$

de $[a, b]_{* \mathbb{R}} \operatorname{com} \sup _{1 \leqslant i \leqslant m}\left(y_{i}-y_{i-1}\right)<\delta$ é refinada, temos que a * $\mathbb{R}$-condição fechada dada por

$$
\left(\exists \zeta \in{ }^{*} \mathbb{R}\right)\left(\zeta>0 \wedge "\left|\sum_{i=1}^{m}{ }^{*} f\left(\rho_{i}\right) \cdot\left(y_{i}-y_{i-1}\right)-\int f\right|<\epsilon\right. \text { para toda }
$$

hiperpartição interna

$$
\begin{array}{r}
Q=\left(y_{0} y_{1} \ldots y_{m}, \rho_{1} \rho_{2} \ldots \rho_{m}\right) \\
\left.\operatorname{de}[a, b]_{* \mathbb{R}} \text { com } \sup _{1 \leqslant i \leqslant m}\left(y_{i}-y_{i-1}\right)<\zeta "\right)
\end{array}
$$

é verdadeira, e, aplicando o PT a essa condição, temos que a condição

$$
\begin{gathered}
(\exists \zeta \in \mathbb{R})\left(\zeta>0 \wedge "\left|\sum_{i=1}^{m} f\left(\rho_{i}\right) \cdot\left(y_{i}-y_{i-1}\right)-\int f\right|<\epsilon\right. \text { para toda partição } \\
Q=\left(y_{0} y_{1} \ldots y_{m}, \rho_{1} \rho_{2} \ldots \rho_{m}\right) \\
\text { de } \left.[a, b]_{\mathbb{R}} \text { com } \sup _{1 \leqslant i \leqslant m}\left(y_{i}-y_{i-1}\right)<\zeta "\right)
\end{gathered}
$$

é verdadeira, provando que $f$ é Riemann-integrável.

Teorema 2.61 (Teorema Fundamental do Cálculo). Seja $f:[a, b]_{\mathbb{R}} \rightarrow \mathbb{R}$ uma função contínua, e seja $F:[a, b]_{\mathbb{R}} \rightarrow \mathbb{R}$ a função dada por $F(x):=\int_{a}^{x} f$. A função $F$ é diferenciável e $F^{\prime}=f$. 
Demonstração. Seja $x \in(a, b)_{\mathbb{R}}$. Para cada número real positivo $h$, seja $P_{h}$ uma hiperpartição refinada interna do intervalo $[x, x+h]_{* \mathbb{R}}$, seja $m_{h}:=\min _{x \leqslant y \leqslant x+h} f(y)$, e seja $M_{h}:=\max _{x \leqslant y \leqslant x+h} f(y)$. Note que $m_{h}$ e $M_{h}$ são números reais bem definidos visto que $f$ é contínua, e temos

$$
\lim _{h \rightarrow 0^{+}} m_{h}=f(x)=\lim _{h \rightarrow 0^{+}} M_{h} .
$$

Suponha que $h$ é um número real positivo. Assim, pelo Teorema 2.60, temos

$$
\frac{F(x+h)-F(x)}{h}=\frac{1}{h} \int_{x}^{x+h} f \sim \frac{1}{h} \cdot \sum_{i=1}^{n}{ }^{*} f\left(\pi_{i}\right) \cdot\left(x_{i}-x_{i-1}\right),
$$

onde o par de sequências $\left(x_{0} x_{1} \ldots x_{n}, \pi_{1} \pi_{2} \ldots \pi_{n}\right)$ representa $P_{h}$. Como $f\left\langle[x, x+h]_{\mathbb{R}}\right\rangle \subset\left[m_{h}, M_{h}\right]_{\mathbb{R}}$, temos ${ }^{*} f\left\langle[x, x+h]_{* \mathbb{R}}\right\rangle \subset\left[m_{h}, M_{h}\right]_{* \mathbb{R}}$, e temos (Teorema 2.53)

$$
\frac{1}{h} \cdot m_{h} \cdot \sum_{i=1}^{n}\left(x_{i}-x_{i-1}\right) \leqslant \frac{1}{h} \cdot \sum_{i=1}^{n} f\left(\pi_{i}\right) \cdot\left(x_{i}-x_{i-1}\right) \leqslant \frac{1}{h} \cdot M_{h} \cdot \sum_{i=1}^{n}\left(x_{i}-x_{i-1}\right) .
$$

resultando em (Corolário 2.54)

$$
m_{h}=\frac{1}{h} \cdot m_{h} \cdot h \leqslant \frac{1}{h} \cdot \sum_{i=1}^{n} f\left(\pi_{i}\right) \cdot\left(x_{i}-x_{i-1}\right) \leqslant \frac{1}{h} \cdot M_{h} \cdot h=M_{h} .
$$

Aplicando a função parte standard st nos três membros dessa inequação, temos

$$
m_{h} \leqslant \frac{1}{h} \int_{x}^{x+h} f \leqslant M_{h}
$$

e, pelo Teorema do Confronto, temos que $\frac{1}{h} \int_{x}^{x+h} f \rightarrow f(x)$ quando $h \rightarrow 0^{+}$. A prova de que $\frac{1}{h} \int_{x}^{x+h} f \rightarrow f(x)$ quando $h \rightarrow 0^{-}$é análoga.

Como anunciado, o aparato matemático descrito informalmente neste capítulo pode ser incorporado em NBG, e o restante desta dissertação será destinada a essa tarefa. Nesse processo de formalização, retificaremos algumas lacunas propositalmente inseridas neste capítulo em prol da simplicidade, como a ausência da construção do monomorfismo não standard, *, e a privação das demonstrações íntegras dos Teoremas 2.3, 2.9, 2.26, 2.27 e 2.31. Também revisitaremos alguns conceitos parcialmente inextricáveis com os quais trabalhamos aqui, como a noção das condições relacionadas a $\mathbb{R}\left(\right.$ ou $\left.{ }^{*} \mathbb{R}\right)$ e a noção dos objetos internos e externos. 



\section{Filtros e Ultrafiltros}

\subsection{A Ideia da Construção de * $\mathbb{R}$}

Uma das construções usuais dos números reais a partir dos números racionais, atribuída a Charles Méray e Georg Cantor, consiste na identificação de cada número real com uma classe de equivalência de sequências de Cauchy em $\mathbb{Q}$, onde duas sequências desse tipo, $\left\{r_{n}\right\}$ e $\left\{s_{n}\right\}$, são equivalentes se, e somente se, temos

$$
\lim _{n \rightarrow \infty}\left(r_{n}-s_{n}\right)=0
$$

Tal relação de equivalência tem em consideração apenas o "valor alvo" de uma sequência de Cauchy "no infinito", ou seja, a classe de equivalência que uma sequência de Cauchy $\left\{r_{n}\right\}$ pertence depende somente do valor residual para o qual essa sequência se aproxima quando $n \rightarrow \infty$, independentemente da forma e rapidez que essa sequência tende a esse valor. Como exemplo, nessa construção de $\mathbb{R}$ as sequências de Cauchy em $\mathbb{Q}$ dadas por

$$
\left\{\frac{n+1}{n}\right\} \text { e }\left\{\frac{n+(-1)^{n}}{n}\right\}
$$

correspondem ao mesmo número real, viz. o número 1, embora a primeira sempre assuma valores maiores que 1 e a segunda se aproxime do valor limite alternando entre valores menores e maiores que 1 .

Neste capítulo, desenvolveremos uma construção dos números hiper-reais análoga à mencionada abstração de Méray e Cantor, a qual foi essencialmente concebida por Edwin Hewitt em 1948 (27) e popularizada por Wilhelmus Luxemburg na década de 1960 (37, 38). Nessa idealização, cada número hiper-real é identificado com uma classe de equivalência de uma sequência de números reais. Em particular, as sequências em $\mathbb{R}$ com limite 0 correspondem aos números infinitesimais em ${ }^{*} \mathbb{R}$, e as sequências ilimitadas em $\mathbb{R}$ correspondem aos números infinitos em ${ }^{*} \mathbb{R}$. A relação de equivalência no conjunto das sequências em $\mathbb{R}$ empregada nessa concepção de ${ }^{*} \mathbb{R}$ é tal que duas sequências de números reais, $\left\{x_{n}\right\}$ e $\left\{y_{n}\right\}$, são equivalentes se, e somente se, o conjunto de índices $n$ tais que $x_{n}=y_{n}$ pertence a um subconjunto de $\mathcal{P}(\mathbb{N})$ cujas propriedades serão estabelecidas nas Seções 3.2, 3.5 e 3.7. Desse modo, a classe de equivalência de uma sequência de números reais depende não somente do possível valor para o qual os elementos dessa sequência assintoticamente se aproximam, mas também da configuração específica de uma subsequência que sumariza o comportamento da sequência em questão. 


\subsection{Filtros}

A noção que introduziremos a seguir é bastante útil no estudo da Topologia, e ela tem aplicações em diversas outras áreas da Matemática.

Definição 3.1. Seja $I$ um conjunto não nulo. Um subconjunto não nulo $\mathcal{F}$ de $\mathcal{P}(I)$ é dito ser um filtro em $I$ se as seguintes condições são observadas:

(F1) Se $X \subset Y \subset I$ e $X \in \mathcal{F}$, então $Y \in \mathcal{F}$;

(F2) Se $X, Y \in \mathcal{F}$, então $X \cap Y \in \mathcal{F}$.

Nesse caso, dizemos que:

- $\mathcal{F}$ é próprio se $\emptyset \notin \mathcal{F}$; caso contrário dizemos que $\mathcal{F}$ é impróprio;

- $\mathcal{F}$ é principal se ele é da forma

$$
\mathcal{F}=\{X \subset I \vdots E \subset X\}
$$

para algum $E \subset I$ não nulo; caso contrário, dizemos que $\mathcal{F}$ é não principal;

- $\mathcal{F}$ é livre se $\bigcap \mathcal{F}=\emptyset$;

- $\mathcal{F}$ é maximal se ele é o único filtro próprio em $I$ que contém $\mathcal{F}$.

A condição (F1) implica que $\mathcal{P}(I)$ é o único filtro impróprio em $I$, e, como filtros são não nulos, temos que o conjunto $I$ pertence a todo filtro em $I$. Todo filtro livre é não principal, e, por (F2), todo filtro próprio em um conjunto finito é principal. Uma interseção de filtros em $I$ é um filtro em $I$.

Há na literatura diversas tentativas de interpretar a noção de um filtro $\mathcal{F}$ em um conjunto $I$ de modo elementar, porém, na visão deste autor, nenhuma delas obteve êxito em especificar uma analogia simples na qual os axiomas (F1) e (F2) são asseguradamente verdadeiros sem que outras propriedades indesejadas também fossem intuitivamente observadas. Por tal razão, não trabalharemos com alguma versão intuitiva da Definição 3.1, embora essa não seja a maneira ideal de introduzir conceitos matemáticos dessa relevância.

Neste Capítulo, assumiremos que $I$ é um conjunto não nulo.

Exemplo 3.2. O conjunto unitário $\{I\}$ é um filtro principal em $I$, o qual é chamado de filtro trivial em $I$.

Exemplo 3.3. Para cada $i \in I$, o conjunto

$$
\mathcal{J}^{i}:=\{X \subset I \vdots i \in X\}
$$


é um filtro principal em $I$. Se um conjunto unitário $\{i\}$ com $i \in I$ pertencer a um filtro próprio $\mathcal{F}$ em $I$, então teremos $\mathcal{J}^{i} \subset \mathcal{F}$ por (F1), e $\{i\} \cap X \neq \emptyset(\forall X \in \mathcal{F})$ por (F2), resultando em $\mathcal{F}=\mathcal{J}^{i}$. Assim, para cada $i \in I$, teremos a igualdade $\mathcal{F}=\mathcal{J}^{i}$ se, e somente se, tivermos $\{i\} \in \mathcal{F}$, implicando que $\mathcal{J}^{i}$ é maximal.

Exemplo 3.4. Seja $\mathcal{T}$ uma topologia em $I$, e seja $J$ um subconjunto não nulo de $I$. O conjunto $\mathcal{T}^{J}$ das $\mathcal{T}$-vizinhanças de $J$ será um filtro em $I$ que não é livre. Temos que $\mathcal{T}$ será uma topologia de Alexandrov ${ }^{1}$ se, e somente se, todo filtro em $I$ da forma $\mathcal{T}^{\{i\}}$ para $i \in I$ for principal.

Exemplo 3.5. Se $I$ for infinito, então o conjunto dos subconjuntos finitos de $I$, o qual é denotado por $\mathcal{P}_{\omega}(I)$, não será um filtro em $I$, visto que ele falhará em satisfazer ao axioma (F1). De fato, teremos $I \notin P_{\omega}(I)$ nesse caso.

Exemplo 3.6. Seja

$$
\mathcal{C}:=\{X \subset I \vdots I-X \text { é finito }\} .
$$

Se $I$ for finito, então $\mathcal{C}$ será o filtro impróprio em $I$.

Suponha que $I$ é infinito. Claramente $I \in \mathcal{C} \neq \emptyset$ e $\emptyset \notin \mathcal{C}$. Se $X \subset Y \subset I$ e $X \in \mathcal{C}$, então teremos $I-Y \subset I-X$, resultando que $I-Y$ é finito e $Y \in \mathcal{C}$. Assim, $\mathcal{C}$ satisfaz ao axioma (F1). Se $X, Y \in \mathcal{C}$, então os conjuntos $I-X, I-Y$ e

$$
(I-X) \cup(I-Y)=I-(X \cap Y)
$$

serão finitos, resultando em $X \cap Y \in \mathcal{C}$, e provando que $\mathcal{C}$ é um filtro próprio em $I$. Tal filtro é chamado de filtro de Fréchet em $I$. Um subconjunto $X \subset I$ é dito ser cofinito se $I-X$ é finito, e por tal razão às vezes $\mathcal{C}$ é chamado de filtro dos cofinitos em $I$.

O filtro $\mathcal{C}$ é livre, pois se $i \in \bigcap \mathcal{C}$, então $i \in I-\{i\} \in \mathcal{C}$, o que é absurdo. Provaremos que $\mathcal{C}$ é o menor filtro livre em $I$. Seja $\mathcal{F}$ um filtro livre em $I$, e suponha que $X \in \mathcal{C}$. Como o complemento $I-X$ é finito, podemos escrever $I-X=\left\{i_{1} i_{2} \ldots i_{n}\right\}$, e, como $\mathcal{F}$ é livre, para cada $i_{k}$ existe um $X_{k} \in \mathcal{F}$ tal que $i_{k} \notin X_{k}$. A interseção $X_{1} \cap \cdots \cap X_{n}$ pertence a $\mathcal{F}$ por $(\mathrm{F} 2)$, e

$$
X_{1} \cap \cdots \cap X_{n} \subset I-\left\{i_{1} i_{2} \ldots i_{n}\right\}=X \in \mathcal{F}
$$

por $(\mathrm{F} 1)$, provando que $\mathcal{C} \subset \mathcal{F}$. De fato, um filtro $\mathcal{F}$ qualquer em $I$ será livre se, e somente se, ele contiver o filtro de Fréchet em $I$, visto que nesse caso teremos $\bigcap \mathcal{F} \subset \bigcap \mathcal{C}=\emptyset$.

Exemplo 3.7. Seja $i$ um elemento de $I$, e seja

$$
\mathcal{C}^{i}:=\{X \subset I \vdots I-X \text { é finito e } i \in X\} .
$$

1 Uma topologia em um conjunto é dita ser uma topologia de Alexandrov se todo ponto do conjunto tem uma menor vizinhança que o contém, ou seja, se a interseção das vizinhanças de qualquer ponto do conjunto é aberta. 
Analogamente ao procedimento realizado no Exemplo 3.6, nota-se que $\mathcal{C}^{i}$ é um filtro em $I$ que não é livre. Se $I$ for finito, então $\mathcal{C}^{i}$ será o filtro principal $\mathcal{J}^{i}$ (Exemplo 3.3) .

Suponha que $I$ é infinito e $\mathcal{C}^{i}$ é principal. Assim, existe um $E \subset I$ tal que $\mathcal{C}^{i}$ é da forma $\{X \subset I \vdots E \subset X\}$. Como $E \in \mathcal{C}^{i}$, temos que $E$ é infinito e $i \in E$, e se $j$ for um elemento de $E$ distinto de $i$, então $E-\{j\}$ será um subconjunto cofinito de $I$ que contém $i$, resultando em $E-\{j\} \in \mathcal{C}^{i}$ e $E \subset E-\{j\}$, o que é absurdo. Portanto, quando $I$ for infinito, o filtro próprio $\mathcal{C}^{i}$ será não principal.

Exemplo 3.8. Suponha que $d: I \times I \rightarrow[0, \infty)_{\mathbb{R}}$ é uma pseudométrica ${ }^{2}$ em $I$ tal que $I$ é $d$-ilimitado. O conjunto

$$
\mathcal{M}^{d}:=\{S \subset I: S=I \text { ou } I-S \text { é } d \text {-limitado }\}
$$

é um filtro próprio em $I$. Se $i \in \bigcap \mathcal{M}^{d}$ e se $r$ for qualquer número real positivo, então a bola aberta

$$
B_{r}(i):=\{j \in I \vdots d(i, j)<r\}
$$

será um subconjunto próprio $d$-limitado de $I$, resultando em $I-B_{r}(i) \in \mathcal{M}^{d}$ e $i \in I-B_{r}(i)$, o que é absurdo. Portanto, o filtro $\mathcal{M}^{d}$ é livre.

Exemplo 3.9. Seja $\mathcal{V}$ uma $\sigma$-algebra em $I$, e seja $\mu: \mathcal{V} \rightarrow[0, \infty]_{\mathbb{R}}$ uma medida não negativa em $I \operatorname{com} \mu(I)>0$. O conjunto

$$
\mathcal{O}^{\mu}:=\{S \in \mathcal{V}: \mu(I-S)=0\}
$$

é um filtro próprio em $I$. Se para cada $i \in I$ tivermos que o conjunto unitário $\{i\}$ é $\mathcal{V}$-mensurável e tem $\mu$-medida zero, então para cada $i \in I$ teremos $I-\{i\} \in \mathcal{O}^{\mu}$, implicando em $\bigcap \mathcal{O}^{\mu}=\emptyset$. Assim, nesse caso, teremos que o filtro $\mathcal{O}^{\mu}$ é livre.

Para cada conjunto $\mathcal{K}$ de subconjuntos de $I$, existe um filtro em $I$ que contém $\mathcal{K}$ e que está contido em todo filtro em $I$ que contém $\mathcal{K}$.

2 Uma pseudométrica em um conjunto não nulo $I$ é uma função $d: I \times I \rightarrow[0, \infty)_{\mathbb{R}}$ tal que para quaisquer $x, y, z \in I$ temos

- $d(x, x)=0$

- $d(x, y)=d(y, x)$;

- $d(x, z) \leqslant d(x, y)+d(y, z)$.
(Distâncias Triviais)

(Simetria)

(Desigualdade Triangular)

Nesse caso, dizemos que um subconjunto $S$ de $I$ é $d$-limitado se existem um $x_{0} \in S$ e um $r \in(0, \infty)_{\mathbb{R}}$ tais que $d\left(x_{0}, x\right)<r(\forall x \in S)$; caso contrário dizemos que $S$ é $d$-ilimitado. Uma métrica em $I$ é uma pseudométrica $d: I \times I \rightarrow[0, \infty)_{\mathbb{R}}$ tal que a condição $d(x, y)=0$ implica $x=y$. 
Definição 3.10. Seja $\mathcal{K}$ um subconjunto de $\mathcal{P}(I)$.

- A interseção dos filtros em $I$ que contêm $\mathcal{K}$ é chamada de filtro em $I$ gerado por $\mathcal{K}$;

- Dizemos que $\mathcal{K}$ satisfaz à Propriedade das Interseções Finitas (PIF) se $\bigcap F \neq \emptyset$ para todo subconjunto finito $F$ de $\mathcal{K}$.

Exemplo 3.11. O filtro em $I$ gerado por $\emptyset$ é o filtro trivial $\{I\}$ (Exemplo 3.2).

Se $\mathcal{K}$ for um conjunto de subconjuntos de $I$, então o filtro em $I$ gerado por $\mathcal{K}$ poderá ser impróprio. A proposição a seguir esclarece precisamente a circunstância em que isso ocorre.

Proposição 3.12. Seja $\mathcal{K} \subset \mathcal{P}(I)$. O filtro $\mathcal{F}$ em I gerado por $\mathcal{K}$ é dado por

$$
\mathcal{F}=\left\{X \subset I \vdots Y_{1} \cap \cdots \cap Y_{n} \subset X \text { para alguma sequência } Y_{1} \ldots Y_{n} \text { em } \mathcal{K}\right\},
$$

o qual será próprio se, e somente se, $\mathcal{K}$ obedecer à PIF.

Demonstração. Nota-se prontamente que $\mathcal{F}$ é um filtro em $I$ que contém $\mathcal{K}$. Se $\mathcal{G}$ for outro filtro em $I$ que contém $\mathcal{K}$, e se $X \in \mathcal{F}$, então existirá uma sequência $Y_{1} \ldots Y_{n}$ em $\mathcal{K}$ com $Y_{1} \cap \cdots \cap Y_{n} \subset X$, teremos $Y_{1} \cap \cdots \cap Y_{n} \in \mathcal{G}$ por (F2), e $X \in \mathcal{G}$ por (F1). Portanto, $\mathcal{F}$ está contido em todo filtro em $I$ que contém $\mathcal{K}$.

Exemplo 3.13. Seja < uma ordem parcial direcionada pela direita ${ }^{3}$ em um subconjunto não nulo $J$ de $I$, e, para cada $i \in J$, seja $i \leqslant$ o conjunto

$$
i \leqslant:=\{j \in J \vdots i \leqslant j\}
$$

O filtro $\mathcal{Z}^{J}$ em $I$ gerado pelo conjunto $\left\{i^{\leqslant}: i \in J\right\}$ é chamado de filtro das caudas de $J$ em $I$.

Se $i_{1} i_{2} \ldots i_{p} \in J$, então, como < é direcionada pela direita, existirá um $j \in J$ com $i_{n} \leqslant j(\forall n)$, e teremos

$$
\emptyset \neq j \leqslant \subset i_{1}^{\leqslant} \cap i_{2}^{\leqslant} \cap \cdots \cap i_{p}^{\leqslant}
$$

Pela Proposição 3.12, o filtro $\mathcal{Z}^{J}$ é próprio e é dado por

$$
\mathcal{Z}^{J}=\{X \subset I \vdots i \leqslant X(\exists i \in J)\}
$$

No caso $I=\mathbb{N}$, temos $\mathcal{Z}^{\mathbb{N}}=\mathcal{C}$ (Exemplo 3.6).

3 Uma ordem parcial < em um conjunto $J$ é dita ser direcionada pela direita se para quaisquer $x, y \in J$ existe um $z \in J$ tal que $x, y \leqslant z$. 
Nota-se diretamente que $\mathcal{Z}^{J}$ será livre se, e somente se, o conjunto parcialmente ordenado $J$ não tiver um maior elemento $k$. Caso contrário, $\mathcal{Z}^{J}$ será o filtro principal $\mathcal{J}^{k}$. Assim, mostramos que o filtro $\mathcal{Z}^{J}$ será não principal se, e somente se, ele for livre.

A partir deste ponto neste capítulo, assumiremos que $\mathcal{F}$ é um filtro próprio em $I$.

Notação 3.14. Seja $C(i)$ uma condição na qual a letra $i$ é uma variável. Usaremos a notação

$$
C(i) \text { q.t.p. }
$$

para denotar a condição

$$
\{i \in I \vdots C(i)\} \in \mathcal{F} .
$$

Quando o filtro $\mathcal{F}$ não está implícito no contexto, tal condição é denotada por $C(i)$ q.t.p. [ $\mathcal{F}]$. Neste trabalho, sempre usaremos a letra $i$ como variável ligada em condições dessa forma.

Nota 3.15. No Capítulo 2, tratamos as condições matemáticas predominantemente como entidades metamatemáticas, as quais podem ser verdadeiras ou falsas. Neste capítulo, elas também serão tratadas como cláusulas completas em ocasiões. Por exemplo, poderemos construir sentenças da forma

$$
\text { "Se } C(i) \text {, então ...", }
$$

onde $C(i)$ representa uma cláusula, em vez de nos limitarmos apenas às sentenças da forma

$$
\text { "Se } C(i) \text { for verdadeira, então ...", }
$$

onde $C(i)$ representa o sujeito da cláusula antecedente da implicação.

Proposição 3.16. Sejam $C_{1}(i)$ e $C_{2}(i)$ duas condições na variável $i$, e seja $C(i, x)$ uma condição nas variáveis $i$ e $x$.

(a) $S e(\forall i \in I) C_{1}(i)$, então $C_{1}(i)$ q.t.p.;

(b) $S e(\forall i \in I)\left(C_{1}(i) \Rightarrow C_{2}(i)\right)$ e se $C_{1}(i)$ q.t.p., então $C_{2}(i)$ q.t.p.;

(c) As condições $C_{1}(i)$ q.t.p. e $C_{2}(i)$ q.t.p. serão verdadeiras se, e somente se, a condição

$$
\left(C_{1}(i) \wedge C_{2}(i)\right) \text { q.t.p. }
$$

for verdadeira; 
(d) Se $\left(A_{i}\right)_{i \in I}$ for uma família de conjuntos não nulos, então as seguintes condições serão equivalentes:
1. $\left(\left(\exists x \in A_{i}\right) C(i, x)\right)$ q.t.p.;
2. $\left(\exists r \in \prod_{k \in I} A_{k}\right)[C(i, r(i))$ q.t.p. $]$;
3. $\left[\left(\exists r \in \prod_{k \in I} A_{k}\right) C(i, r(i))\right]$ q.t.p.

Demonstração.

(a) Basta notar que $I \in \mathcal{F}$.

(b) $\operatorname{Temos}\left\{i \in I \vdots C_{1}(i)\right\} \in \mathcal{F} \mathrm{e}$

$$
\left\{i \in I \vdots C_{1}(i)\right\} \subset\left\{i \in I \vdots C_{2}(i)\right\}
$$

resultando em $\left\{i \in I \vdots C_{2}(i)\right\} \in \mathcal{F}$ pelo axioma $(\mathrm{F} 1)$.

(c) Se $C_{1}(i)$ q.t.p. e $C_{2}(i)$ q.t.p., então os conjuntos $\left\{i \in I \vdots C_{1}(i)\right\}$ e $\left\{i \in I \vdots C_{2}(i)\right\}$ pertencerão a $\mathcal{F}$, e, pelo axioma (F2), a interseção

$$
\left\{i \in I \vdots C_{1}(i)\right\} \cap\left\{i \in I \vdots C_{2}(i)\right\}=\left\{i \in I \vdots C_{1}(i) \wedge C_{2}(i)\right\}
$$

pertencerá a $\mathcal{F}$. A prova da implicação recíproca segue pela letra $(b)$.

(d) Como cada $A_{i}$ é não nulo, nota-se prontamente que as condições

$$
\left(\exists x \in A_{i}\right) C(i, x) \text { e }\left(\exists r \in \prod_{k \in I} A_{k}\right) C(i, r(i))
$$

são equivalentes para cada $i \in I$, implicando que as condições 1 e 3 são equivalentes.

Se $r \in \prod_{k \in I} A_{k}$ for tal que $C(i, r(i))$ q.t.p., então teremos

$$
\left\{i \in I \vdots\left(\exists x \in A_{i}\right) C(i, x)\right\} \supset\{i \in I \vdots C(i, r(i))\} \in \mathcal{F},
$$

resultando em $\left(\left(\exists x \in A_{i}\right) C(i, x)\right)$ q.t.p. por (F1), e provando que 2 implica 1.

Suponha que a condição 1 é verdadeira, e seja $J$ o conjunto

$$
J:=\left\{i \in I \vdots\left(\exists x \in A_{i}\right) C(i, x)\right\} .
$$


Assim, temos $J \in \mathcal{F}$. Para cada $i \in J$, seja $r(i)$ um elemento de $A_{i} \operatorname{com} C(i, r(i))$, e para cada $i \in I-J$, seja $r(i)$ um elemento qualquer de $A_{i}$. Portanto, temos

$$
\{i \in I \vdots C(i, r(i))\}=J \in \mathcal{F},
$$

provando que 1 implica 2.

\subsection{Produtos Reduzidos}

Considerando uma família de conjuntos $\left\{M_{i}\right\}_{i \in I}$ com índices em $I$, temos que o filtro $\mathcal{F}$ em $I$ canonicamente induz uma relação de equivalência no produto cartesiano $\prod_{i \in I} M_{i}$.

Definição 3.17. Seja $\left\{M_{i}\right\}_{i \in I}$ uma família de conjuntos. Denotaremos por $=_{M_{i}, \mathcal{F}}$ a relação binária no produto cartesiano $\prod_{i \in I} M_{i}$ definida de modo que, para quaisquer $r, s \in \prod_{i \in I} M_{i}$, teremos $r={ }_{M_{i}, \mathcal{F}} s$ se, e somente se, tivermos $r(i)=s(i)$ q.t.p.

O resultado a seguir segue diretamente da Proposição 3.16.

Corolário 3.18. Seja $\left\{M_{i}\right\}_{i \in I}$ uma família de conjuntos. A relação $=_{M_{i}, \mathcal{F}}$ é uma relação de equivalência em $\prod_{i \in I} M_{i}$.

Notação 3.19. Para cada $r \in \prod_{i \in I} M_{i}$, a classe de equivalência

$$
\begin{aligned}
r /={ }_{M_{i}, \mathcal{F}} & =\left\{s \in \prod_{i \in I} M_{i} \vdots r={ }_{M_{i}, \mathcal{F}} s\right\} \\
& =\left\{s \in \prod_{i \in I} M_{i} \vdots r(i)=s(i) \text { q.t.p. }\right\}
\end{aligned}
$$

é dita ser o limite de $r$ módulo $\left(M_{i}, \mathcal{F}\right)$, e ela é denotada por $\lim _{M_{i}, \mathcal{F}} r(i)$.

Proposição 3.20. Sejam $\left\{M_{i}\right\}_{i \in I} e\left\{N_{i}\right\}_{i \in I}$ duas famílias de conjuntos não nulos tais que $M_{i} \subset N_{i}(\forall i \in I)$, e seja $s \in \prod_{i \in I} N_{i}$ tal que $s(i) \in M_{i}$ q.t.p. $\quad$ Existe um $r \in \prod_{i \in I} M_{i}$ tal que $r(i)=s(i)$ q.t.p. 
Demonstração. Como cada conjunto $M_{i}$ é não nulo, existe um $t \in \prod_{i \in I} M_{i}$ (Teorema B.26). Seja $J:=\left\{i \in I \vdots s(i) \in M_{i}\right\}$ e seja $r$ a função com domínio $I$ dada por

$$
r(i):= \begin{cases}s(i) & \text { se } i \in J \\ t(i) & \text { se } i \in I-J\end{cases}
$$

Assim, $J$ está contido no conjunto $\{i \in I \vdots r(i)=s(i)\}$, e, como $J \in \mathcal{F}$, temos $r(i)=s(i)$ q.t.p. por (F1).

Sob as condições da Proposição 3.20, seja $r^{\prime} \in \prod_{i \in I} M_{i}$. Nota-se que $r^{\prime}(i)=s(i)$ q.t.p. se, e somente se, $r^{\prime}(i)=r(i)$ q.t.p. (Proposição 3.16, Letras $(b)$ e $(c)$ ), resultando em

$$
\lim _{M_{i}, \mathcal{F}} r(i)=\left\{r^{\prime} \in \prod_{i \in I} M_{i} \vdots r^{\prime}(i)=s(i) \text { q.t.p. }\right\} .
$$

Portanto, a classe de equivalência de $r$ em relação a $=_{M_{i}, \mathcal{F}}$ é determinada por $s$, justificando a seguinte notação:

Notação 3.21. Em situações análogas à da Proposição 3.20, a classe de equivalência $\lim _{M_{i}, \mathcal{F}} r(i)$ poderá ser denotada por $\lim _{M_{i}, \mathcal{F}} s(i)$ por abuso de linguagem.

Até agora nesta seção, obtivemos uma sistematização de um processo que gera um conjunto quociente

$$
\left(\prod_{i \in I} M_{i}\right) /={ }_{M_{i}, \mathcal{F}}
$$

a partir de uma família de conjuntos $\left\{M_{i}\right\}_{i \in I}$ com índices em $I$. Esse processo pode ser generalizado, ${ }^{4}$ de modo que se $L$ for uma assinatura (Definição A.5) e se $\left\{M_{i}\right\}_{i \in I}$ for uma família de $L$-estruturas (Definição A.23) com índices em $I$, então poderemos canonicamente definir uma $L$-estrutura cujo universo é o quociente do produto cartesiano da família $\left\{\left\|M_{i}\right\|\right\}_{i \in I}$ módulo $=_{\left\|M_{i}\right\|, \mathcal{F}}$.

Até o restante deste capítulo, assumiremos que $L$ é uma assinatura e $\left\{M_{i}\right\}_{i \in I}$ é uma família de $L$-estruturas com índices em $I$, e, por abuso de linguagem, denotaremos o produto cartesiano $\prod_{i \in I}\left\|M_{i}\right\|$ por $\prod_{i \in I} M_{i}$.

Antes de definir a nova $L$-estrutura mencionada, devemos levar em conta outra decorrência imediata da Proposição 3.16:

Corolário 3.22. Se $r_{1} r_{2} \ldots r_{n}$ e $s_{1} s_{2} \ldots s_{n}$ forem sequências de funções pertencentes a $\prod_{i \in I} M_{i}$ tais que $r_{k}={ }_{M_{i}, \mathcal{F}} s_{k}(\forall k)$, então as seguintes condições serão verdadeiras:

4 Conjuntos são $\emptyset$-estruturas. 
(a) Para cada símbolo relacional n-ário $P$ em L, teremos

$$
\left(r_{1}(i) \ldots r_{n}(i)\right) \in P^{M_{i}} \text { q.t.p. }
$$

se, e somente se, tivermos

$$
\left(s_{1}(i) \ldots s_{n}(i)\right) \in P^{M_{i}} \text { q.t.p.; }
$$

(b) Para cada símbolo funcional n-ário $G$ em L, teremos

$$
G^{M_{i}}\left(r_{1}(i) \ldots r_{n}(i)\right)=G^{M_{i}}\left(s_{1}(i) \ldots s_{n}(i)\right) \text { q.t.p. }
$$

Definição 3.23. O produto reduzido de $\left\{M_{i}\right\}_{i \in I}$ módulo $\mathcal{F}$ é a $L$-estrutura $N$ definida como segue:

1. O universo de $N$ é definido por

$$
\begin{aligned}
\|N\| & :=\left(\prod_{i \in I} M_{i}\right) /=_{M_{i}, \mathcal{F}} \\
& =\left\{\lim _{M_{i}, \mathcal{F}} r(i) \vdots r \in \prod_{i \in I} M_{i}\right\} ;
\end{aligned}
$$

2. Para cada símbolo relacional $n$-ário $P$ em $L$, definimos a relação $n$-ária $P^{N}$ em $\|N\|$ tal que

$$
\left(\lim _{M_{i}, \mathcal{F}} r_{1}(i) \ldots \lim _{M_{i}, \mathcal{F}} r_{n}(i)\right) \in P^{N}
$$

se

$$
\left(r_{1}(i) \ldots r_{n}(i)\right) \in P^{M_{i}} \text { q.t.p. }
$$

Tal relação é bem definida (Corolário 3.22, Letra $(a)$ );

3. Para cada símbolo funcional $n$-ário $G$ em $L$, definimos a função $G^{N}:\|N\|^{n} \rightarrow\|N\|$ dada por

$$
G^{N}\left(\lim _{M_{i}, \mathcal{F}} r_{1}(i) \ldots \lim _{M_{i}, \mathcal{F}} r_{n}(i)\right):=\lim _{M_{i}, \mathcal{F}} G^{M_{i}}\left(r_{1}(i) \ldots r_{n}(i)\right)
$$

Tal função é bem definida (Corolário 3.22, Letra (b));

4. Para cada símbolo de constante $c$ em $L$, definimos $c^{N}:=\lim _{M_{i}, \mathcal{F}} c^{M_{i}}$.

Essa $L$-estrutura é denotada por $\prod_{\mathcal{F}} M_{i}$. Se $\left\{M_{i}\right\}_{i \in I}$ é uma família constante $\operatorname{com} M_{i}=M(\forall i \in I)$, então o produto reduzido $\prod_{\mathcal{F}} M$ é dito ser a potência reduzida de $M$ módulo $\mathcal{F}$, e a função $d: M \rightarrow \prod_{\mathcal{F}} M$ dada por $d(x):=\lim _{M, \mathcal{F}} x$ é dita ser canônica. 
Convenciona-se (Definição A.23) que o símbolo relacional binário de igualdade, $=$, sempre deve ser interpretado em uma $L$-estrutura qualquer $M$ da seguinte forma

$$
={ }^{M}:=\left\{(x, y) \in\|M\|^{2}: x=y\right\} .
$$

No caso do produto reduzido $\prod_{\mathcal{F}} M_{i}$, temos que essa convenção vai ao encontro do item 2 da Definição 3.23, visto que a condição

$$
\lim _{M_{i}, \mathcal{F}} r_{1}(i)=\lim _{M_{i}, \mathcal{F}} r_{2}(i)
$$

é equivalente à condição $\left(r_{1}(i), r_{2}(i)\right) \in={ }^{M_{i}}$ q.t.p., onde $r_{1}, r_{2} \in \prod_{i \in I} M_{i}$.

Exemplo 3.24. O produto direto da família de $L$-estruturas $\left\{M_{i}\right\}_{i \in I}$ é a $L$-estrutura $N$ definida por:

- $\|N\|:=\prod_{i \in I} M_{i}$

- Para cada símbolo relacional $n$-ário $P$ em $L$, definimos a relação $n$-ária $P^{N}$ em $\|N\|$ tal que

$$
\left(r_{1} \ldots r_{n}\right) \in P^{N}
$$

se

$$
\left(r_{1}(i) \ldots r_{n}(i)\right) \in P^{M_{i}}(\forall i \in I)
$$

- Para cada símbolo funcional $n$-ário $G$ em $L$, definimos a função $G^{N}:\|N\|^{n} \rightarrow\|N\|$ tal que, para quaisquer $r_{1} \ldots r_{n}$ em $\|N\|$, a imagem $G^{N}\left(r_{1} \ldots r_{n}\right)$ é a função $i \mapsto G^{M_{i}}\left(r_{1}(i) \ldots r_{n}(i)\right)$ com domínio $I$;

- Para cada símbolo de constante $c$ em $L$, definimos $c^{N}$ como sendo a função $i \mapsto c^{M_{i}}$ com domínio $I$.

Se $\mathcal{F}$ for o filtro trivial $\{I\}$ em $I$, então o limite de cada $r \in \prod_{i \in I} M_{i}$ módulo $\left(M_{i}, \mathcal{F}\right)$ será o conjunto unitário $\{r\}$, e a função $f: N \rightarrow \prod_{\mathcal{F}} M_{i}$ dada por $f(r):=\{r\}$ será um isomorfismo entre $L$-estruturas. Desse modo, temos que os produtos reduzidos generalizam os produtos diretos.

Exemplo 3.25. Suponha que o conjunto $I$ é infinito e é tal que para cada número natural $n$ existe um subconjunto cofinito $J_{n}$ de $I$ tal que cada $L$-estrutura $M_{i}$ com $i \in J_{n}$ tem no mínimo $n$ elementos. No Exemplo 3.48, mostraremos que se $\mathcal{F}$ for livre, então o produto reduzido $\prod_{\mathcal{F}} M_{i}$ será infinito. 
Teorema 3.26 (Termos Interpretados por Produtos Reduzidos). Se $t\left(x_{1} \ldots x_{n}\right)$ for um L-termo (Definição A.13) e se $r_{1} \ldots r_{n} \in \prod_{i \in I} M_{i}$, então

$$
t^{\prod_{\mathcal{F}} M_{i}}\left(\lim _{M_{i}, \mathcal{F}} r_{1}(i) \ldots \lim _{M_{i}, \mathcal{F}} r_{n}(i)\right)=\lim _{M_{i}, \mathcal{F}} t^{M_{i}}\left(r_{1}(i) \ldots r_{n}(i)\right)
$$

Demonstração. Procederemos por indução sobre a complexidade de $t$. O resultado é imediato quando $t$ é uma variável ou um símbolo de constante em $L$. Suponha que $t$ tem complexidade $k$ positiva, e que o teorema já foi provado para termos de complexidade menor que $k$. Assim, $t$ será da forma

$$
t\left(x_{1} \ldots x_{n}\right)=G\left(t_{1}\left(x_{1} \ldots x_{n}\right), \ldots, t_{m}\left(x_{1} \ldots x_{n}\right)\right)
$$

onde $G$ é um símbolo relacional $m$-ário em $L$ e $t_{1} \ldots t_{m}$ são $L$-termos abertos nas variáveis $x_{1} \ldots x_{n}$. Definindo $N:=\prod_{\mathcal{F}} M_{i}$, temos

$$
\begin{aligned}
t^{N}\left(\lim _{M_{i}, \mathcal{F}} r_{1}(i) \ldots \lim _{M_{i}, \mathcal{F}} r_{n}(i)\right) & =G^{N}\left(t_{1}^{N}\left(\lim _{M_{i}, \mathcal{F}} r_{1}(i) \ldots \lim _{M_{i}, \mathcal{F}} r_{n}(i)\right), \ldots, t_{m}^{N}\left(\lim _{M_{i}, \mathcal{F}} r_{1}(i) \ldots \lim _{M_{i}, \mathcal{F}} r_{n}(i)\right)\right) \\
& =G^{N}\left(\lim _{M_{i}, \mathcal{F}} t_{1}^{M_{i}}\left(r_{1}(i) \ldots r_{n}(i)\right), \ldots, \lim _{M_{i}, \mathcal{F}} t_{m}^{M_{i}}\left(r_{1}(i) \ldots r_{n}(i)\right)\right) \\
& =\lim _{M_{i}, \mathcal{F}} G^{M_{i}}\left(t_{1}^{M_{i}}\left(r_{1}(i) \ldots r_{n}(i)\right), \ldots, t_{m}^{M_{i}}\left(r_{1}(i) \ldots r_{n}(i)\right)\right) \\
& =\lim _{M_{i}, \mathcal{F}} t^{M_{i}}\left(r_{1}(i) \ldots r_{n}(i)\right)
\end{aligned}
$$

onde a hipótese de indução foi aplicada na transição da primeira linha para a segunda. Portanto, o teorema é válido para termos de complexidade $k$, e, pelo Princípio da Indução, o resultado está demonstrado.

\subsection{Limites Generalizados}

Podemos definir subconjuntos do universo de um produto reduzido $\prod_{\mathcal{F}} M_{i}$ a partir de famílias $\left\{A_{i}\right\}_{i \in I}$ de conjuntos com quase todo conjunto $A_{i}$ contido no universo da respectiva $L$-estrutura $M_{i}$.

Definição 3.27. Seja $\left\{A_{i}\right\}_{i \in I}$ uma família de conjuntos tal que $A_{i} \subset\left\|M_{i}\right\|$ q.t.p. O limite integral de $\left\{A_{i}\right\}_{i \in I}$ módulo $\left(M_{i}, \mathcal{F}\right)$ é o subconjunto de $\prod_{\mathcal{F}} M_{i}$ definido por

$$
\left\{\lim _{M_{i}, \mathcal{F}} r(i) \vdots r \in \prod_{i \in I} M_{i} \text { e } r(i) \in A_{i} \text { q.t.p. }\right\},
$$

o qual é denotado por $\lim _{M_{i}, \mathcal{F}} A_{i}$.

Exemplo 3.28. Se $A_{i}=\emptyset$ para todo $i \in I$, então teremos $\lim _{M_{i}, \mathcal{F}} A_{i}=\emptyset$. 
A proposição a seguir mostra que o limite integral $\lim _{M_{i}, \mathcal{F}} A_{i}$ é uma cópia do produto reduzido $\prod_{\mathcal{F}} A_{i}$ de $\emptyset$-estruturas (i.e., conjuntos) quando cada $A_{i}$ é não nulo.

Proposição 3.29. Seja $\left\{A_{i}\right\}_{i \in I}$ uma família de conjuntos não nulos tal que $A_{i} \subset\left\|M_{i}\right\|$ q.t.p. A função $u: \prod_{\mathcal{F}} A_{i} \rightarrow \lim _{M_{i}, \mathcal{F}} A_{i}$ dada por

$$
u\left(\lim _{A_{i}, \mathcal{F}} r(i)\right):=\lim _{M_{i}, \mathcal{F}} r(i)
$$

é bem definida e é bijetora.

Demonstração. Como $\prod_{i \in I} A_{i} \subset \prod_{i \in I} M_{i}$, nota-se que $u$ é bem definida. A injetividade de $u$ é imediata, e, como cada conjunto $A_{i}$ é não nulo, a sobrejetividade de $u$ segue pela Proposição 3.20 .

Relações $n$-árias cujas $n$-tuplas têm as $j$-ésimas coordenadas pertencentes a produtos reduzidos $\prod_{\mathcal{F}} N_{i}^{j}$ para cada $j \in[1, n]_{\mathbb{N}}$ são canonicamente definidas a partir de famílias $\left\{R_{i}\right\}_{i \in I}$ de relações $n$-árias que satisfazem a certas condições, como veremos na definição a seguir.

Definição 3.30. Seja $\left\{N_{i}^{j}\right\}_{\substack{i \in j \leqslant n \\ 1 \leqslant I}}$ uma família dupla de $L$-estruturas, seja $\left\{A_{i}^{j}\right\}_{\substack{i \leqslant j \leqslant n \\ 1 \leqslant j}}$ uma família dupla de conjuntos com $A_{i}^{j} \subset\left\|N_{i}^{j}\right\|$ q.t.p. $(\forall j)$, e seja $\left\{R_{i}\right\}_{i \in I}$ uma família de relações $n$-árias com $R_{i} \subset A_{i}^{1} \times A_{i}^{2} \times \cdots \times A_{i}^{n}$ q.t.p. $\quad$ O limite relacional de $\left\{R_{i}\right\}_{i \in I}$ módulo $\left(\left\{N_{i}^{j}\right\}_{1 \leqslant j \leqslant n}, \mathcal{F}\right)$ é a relação $n$-ária

$$
\lim _{N_{i}^{j}, \mathcal{F}} R_{i} \subset\left(\lim _{N_{i}^{1}, \mathcal{F}} A_{i}^{1}\right) \times\left(\lim _{N_{i}^{2}, \mathcal{F}} A_{i}^{2}\right) \times \cdots \times\left(\lim _{N_{i}^{n}, \mathcal{F}} A_{i}^{n}\right)
$$

definida por

$$
\left(\lim _{N_{i}^{1}, \mathcal{F}} r_{1}(i) \ldots \lim _{N_{i}^{n}, \mathcal{F}} r_{n}(i)\right) \in \lim _{N_{i}^{j}, \mathcal{F}} R_{i}: \Leftrightarrow\left(r_{1}(i) \ldots r_{n}(i)\right) \in R_{i} \text { q.t.p. }
$$

onde $r_{j} \in \prod_{i \in I} N_{i}^{j}(\forall j)$. O limite relacional $\lim _{N_{i}^{j}, \mathcal{F}} R_{i}$ é uma relação $n$-ária bem definida (Proposição 3.16).

Proposição 3.31. Seja $\left\{N_{i}^{j}\right\}_{\substack{i \in j \leqslant n+1 \\ 1 \leqslant j \leqslant n+1}}$ uma familia dupla de L-estruturas, seja $\left\{A_{i}^{j}\right\}_{\substack { 1 \leqslant j \leqslant n+1 \\ \begin{subarray}{c}{i \in I \\ \text { I }{ 1 \leqslant j \leqslant n + 1 \\ \begin{subarray} { c } { i \in I \\ \text { I } } }\end{subarray}}$ uma família dupla de conjuntos com $A_{i}^{j} \subset\left\|N_{i}^{j}\right\|$ q.t.p. $(\forall j)$, e seja $\left\{f_{i}\right\}_{i \in I}$ uma família 
de funções $n$-árias com $f_{i}: A_{i}^{1} \times A_{i}^{2} \times \cdots \times A_{i}^{n} \rightarrow A_{i}^{n+1}$ q.t.p. $\quad O$ limite relacional $d a$ família $\left\{f_{i}\right\}_{i \in I}$ módulo $\left(\left\{N_{i}^{j}\right\}_{\substack{i \leqslant j \leqslant n+1 \\ i \in I}}, \mathcal{F}\right)$ é uma função n-ária do tipo

$$
\lim _{N_{i}^{j}, \mathcal{F}} f_{i}:\left(\lim _{N_{i}^{1}, \mathcal{F}} A_{i}^{1}\right) \times\left(\lim _{N_{i}^{2}, \mathcal{F}} A_{i}^{2}\right) \times \cdots \times\left(\lim _{N_{i}^{n}, \mathcal{F}} A_{i}^{n}\right) \rightarrow \lim _{N_{i}^{n+1}, \mathcal{F}} A_{i}^{n+1}
$$

dada por

$$
\left(\lim _{N_{i}^{j}, \mathcal{F}} f_{i}\right)\left(\lim _{N_{i}^{1}, \mathcal{F}} r_{1}(i) \ldots \lim _{N_{i}^{n}, \mathcal{F}} r_{n}(i)\right):=\lim _{N_{i}^{n+1}, \mathcal{F}} f_{i}\left(r_{1}(i) \ldots r_{n}(i)\right),
$$

onde $f_{i}\left(r_{1}(i) \ldots r_{n}(i)\right)$ pode ser definido como sendo qualquer objeto quando $i$ for tal que $\left(r_{1}(i) \ldots r_{n}(i)\right) \notin \operatorname{dom}\left(f_{i}\right)$ (Notação 3.21).

Demonstração. Pela definição dos limites relacionais, temos

$$
\lim _{N_{i}^{j}, \mathcal{F}} f_{i} \subset\left(\lim _{N_{i}^{1}, \mathcal{F}} A_{i}^{1}\right) \times\left(\lim _{N_{i}^{2}, \mathcal{F}} A_{i}^{2}\right) \times \cdots \times\left(\lim _{N_{i}^{n}, \mathcal{F}} A_{i}^{n}\right) \times\left(\lim _{N_{i}^{n+1}, \mathcal{F}} A_{i}^{n+1}\right) .
$$

Sejam $r_{1} r_{2} \ldots r_{n}$ funções tais que, para cada $j \in[1, n]_{\mathbb{N}}$, temos $r_{j} \in \prod_{i \in I} N_{i}^{j}$ e $r_{j}(i) \in A_{i}^{j}$ q.t.p. $\quad$ Assim, tendo em vista a Proposição 3.16, temos

$$
\left(r_{1}(i) \ldots r_{n}(i)\right) \in A_{i}^{1} \times A_{i}^{2} \times \cdots \times A_{i}^{n} \text { q.t.p. }
$$

e

$$
\left(r_{1}(i) \ldots r_{n}(i), f_{i}\left(r_{1}(i) \ldots r_{n}(i)\right)\right) \in f_{i} \text { q.t.p. }
$$

resultando em

$$
\left(\lim _{N_{i}^{1}, \mathcal{F}} r_{1}(i) \ldots \lim _{N_{i}^{n}, \mathcal{F}} r_{n}(i), \lim _{N_{i}^{n+1}, \mathcal{F}} f_{i}\left(r_{1}(i) \ldots r_{n}(i)\right)\right) \in \lim _{N_{i}^{j}, \mathcal{F}} f_{i} .
$$

Se $s_{1}, s_{2} \in \prod_{i \in I} N_{i}^{n+1}$ com $s_{1}(i) \in A_{i}^{n+1}$ q.t.p. e $s_{2}(i) \in A_{i}^{n+1}$ q.t.p., e se as $(n+1)$-tuplas

$$
\left(\lim _{N_{i}^{1}, \mathcal{F}} r_{1}(i) \ldots \lim _{N_{i}^{n}, \mathcal{F}} r_{n}(i), \lim _{N_{i}^{n+1}, \mathcal{F}} s_{1}(i)\right) \text { e }\left(\lim _{N_{i}^{1}, \mathcal{F}} r_{1}(i) \ldots \lim _{N_{i}^{n}, \mathcal{F}} r_{n}(i), \lim _{N_{i}^{n+1}, \mathcal{F}} s_{2}(i)\right)
$$

pertencerem à relação $\lim _{N_{i}^{j}, \mathcal{F}} f_{i}$, então

$$
\left(\left(r_{1}(i) \ldots r_{n}(i), s_{1}(i)\right) \in f_{i} \wedge\left(r_{1}(i) \ldots r_{n}(i), s_{2}(i)\right) \in f_{i}\right) \text { q.t.p. }
$$

e, como ( $f_{i}$ é uma função) q.t.p., teremos $s_{1}(i)=s_{2}(i)$ q.t.p. e $\lim _{N_{i}^{n+1}, \mathcal{F}} s_{1}(i)=\lim _{N_{i}^{n+1}, \mathcal{F}} s_{1}(i)$, provando que $\lim _{N_{i}^{j}, \mathcal{F}} f_{i}$ é uma função.

Sob as condições da Proposição 3.31, dizemos que a função $n$-ária $\lim _{N^{j} \mathcal{F}} f_{i}$ é o limite funcional de $\left\{f_{i}\right\}_{i \in I}$ módulo $\left(\left\{N_{i}^{j}\right\}_{\substack{i \leqslant j \leqslant n+1 \\ 1 \in I}}, \mathcal{F}\right)$. 


\subsection{Ultrafiltros}

Para cada $X \subset I$, temos que $X$ e o seu complemento $I-X$ não podem simultaneamente pertencer ao filtro próprio $\mathcal{F}$, visto que isso implicaria em $\emptyset=X \cap(I-X) \in \mathcal{F}$ pelo axioma (F2). Nesta seção, investigaremos os filtros $\mathcal{U}$ em $I$ que são saturados no sentido que o complemento $I-X$ de um subconjunto $X$ de $I$ pertencerá ao filtro $\mathcal{U}$ sempre que $X$ não pertencer a $\mathcal{U}$.

Definição 3.32. Um filtro próprio $\mathcal{U}$ sobre um conjunto não nulo $I$ é dito ser um ultrafiltro em $I$ se para cada $X \subset I$ temos

(U) Se $X \notin \mathcal{U}$, então $I-X \in \mathcal{U}$.

O limite $\lim _{M_{i}, \mathcal{U}} r(i)$ é chamado de ultralimite de $r$ módulo $\left(M_{i}, \mathcal{U}\right)$ para cada $r \in \prod_{i \in I} M_{i}$, o produto reduzido $\prod_{\mathcal{U}} M_{i}$ é chamado de ultraproduto de $\left\{M_{i}\right\}_{i \in I}$ módulo $\mathcal{U}$, e cada um dos três tipos de limites generalizados módulo $\left(M_{i}, \mathcal{U}\right)$ são ditos serem ultralimites generalizados. Se $\left\{M_{i}\right\}_{i \in I}$ é uma família constante com $M_{i}=M(\forall i \in I)$, então o ultraproduto $\prod_{\mathcal{U}} M$ é dito ser a ultrapotência de $M$ módulo $\mathcal{U}$.

O axioma (U) configura os ultrafiltros de modo que eles sejam filtros próprios que compreendem a maior quantidade possível de subconjuntos de $I$. O teorema a seguir mostra que os ultrafiltros podem ser caracterizados por outras propriedades de saturação.

Teorema 3.33. As seguintes condições são equivalentes:

(a) $\mathcal{F}$ é um ultrafiltro em $I$;

(b) Para cada $X \subset I$, teremos $X \in \mathcal{F}$ se, e somente se, $I-X \notin \mathcal{F}$;

(c) Para quaisquer subconjuntos $X_{1} \ldots X_{n}$ de I, se tivermos

$$
X_{1} \cup \cdots \cup X_{n} \in \mathcal{F},
$$

então algum $X_{i}$ pertencerá a $\mathcal{F}$;

(d) $\mathcal{F}$ é maximal (Definição 3.1).

Demonstração. Provaremos separadamente que (a) é equivalente a cada uma das condições $(b),(c)$ e $(d)$. Claramente, temos $(b) \Rightarrow(a)$.

$(a) \Rightarrow(b)$ : Seja $X$ um subconjunto de $I$. Se $I-X \notin \mathcal{F}$, então

$$
X=I-(I-X) \in \mathcal{F},
$$

e se $I-X \in \mathcal{F}$, então $X \notin \mathcal{F}$ por (F2). 
$(a) \Rightarrow(c):$ Sejam $X_{1} \ldots X_{n}$ subconjuntos de $I$ com $\bigcup_{i=1}^{n} X_{i} \in \mathcal{F}$. Se $X_{i} \notin \mathcal{F}(\forall i)$, então $I-X_{i} \in \mathcal{F}(\forall i)$, e por $(\mathrm{F} 2)$ teremos

$$
I-\bigcup_{i=1}^{n} X_{i}=\bigcap_{i=1}^{n}\left(I-X_{i}\right) \in \mathcal{F},
$$

o que é absurdo.

$(c) \Rightarrow(a):$ Se $X$ for um subconjunto de $I$, então $X \cup(I-X)=I \in \mathcal{F}$, implicando que $X \in \mathcal{F}$ ou $I-X \in \mathcal{F}$.

$(a) \Rightarrow(d)$ : Se $\mathcal{E}$ for um filtro próprio em $I$ que contém $\mathcal{F}$, e se $X \in \mathcal{E}-\mathcal{F}$, então teremos $I-X \in \mathcal{F} \subset \mathcal{E}$, resultando em

$$
\emptyset=X \cap(I-X) \in \mathcal{E}
$$

por (F2), o que é absurdo.

$(d) \Rightarrow(a)$ : Seja $X \subset I \operatorname{com} X \notin \mathcal{F}$, e seja $\mathcal{K}:=\mathcal{F} \cup\{I-X\}$. Provaremos que $\mathcal{F}$ é um ultrafiltro em $I$, e, para isso, basta provarmos que $I-X \in \mathcal{F}$. Por (F2), uma interseção finita de elementos de $\mathcal{K}$ ou pertence a $\mathcal{F}$ ou é da forma $Y \cap(I-X)$ para algum $Y \in \mathcal{F}$. No primeiro caso, tal interseção finita é não nula visto que $\mathcal{F}$ é um filtro próprio. No segundo caso, temos $Y \cap(I-X) \neq 0$ pois caso contrário teríamos $Y \subset X$ e $X \in \mathcal{F}$ por (F1), o que seria absurdo. Portanto, interseções finitas de elementos de $\mathcal{K}$ são não nulas, e, pela Proposição 3.12, o filtro $\mathcal{H}$ em $I$ gerado por $\mathcal{K}$ é próprio. Como $\mathcal{F}$ é maximal, temos $\mathcal{F}=\mathcal{H}$ e $I-X \in \mathcal{F}$.

Chamaremos de (U') a condição expressa na letra (b) do Teorema 3.33, a qual pode ser vista como uma alternativa simples a (U) para axiomatizar os ultrafiltros.

Exemplo 3.34. O filtro trivial $\{I\}$ em $I$ será um ultrafiltro em $I$ se, e somente se, $I$ for um conjunto unitário, visto que se $i \in I$ e $I-\{i\} \neq \emptyset$, então os conjuntos $\{i\}$ e $I-\{i\}$ não pertencerão a $\{I\}$, contradizendo (U').

Exemplo 3.35. Para cada $i \in I$, o filtro próprio $\mathcal{J}^{i}$ (Exemplo 3.3) é ultrafiltro em $I$.

Exemplo 3.36. Suponha que $I$ é infinito. Assim, existe uma função injetora $f: \mathbb{N} \rightarrow I$, e os conjuntos $2 \mathbb{N}, f\langle 2 \mathbb{N}\rangle, 2 \mathbb{N}-1$ e $f\langle 2 \mathbb{N}-1\rangle$ têm o mesmo cardinal $\omega$, implicando que todos eles são infinitos. O complemento de $f\langle 2 \mathbb{N}\rangle$ em $I$ também é infinito visto que $f\langle 2 \mathbb{N}-1\rangle \subset I-f\langle 2 \mathbb{N}\rangle$. Portanto, $f\langle 2 \mathbb{N}\rangle$ e $I-f\langle 2 \mathbb{N}\rangle$ não pertencem ao filtro de Fréchet em $I$ (Exemplo 3.6), provando que $\mathcal{C}$ não é um ultrafiltro em $I$ pelo axioma (U'). 
Exemplo 3.37. Seja $i$ um elemento de $I$. Se $I$ for finito, então o filtro $\mathcal{C}^{i}$ em $I$ (Exemplo 3.7) será o filtro principal $\mathcal{J}^{i}$, o qual claramente é um ultrafiltro em $I$, e se $I$ for infinito, então, analogamente à prova mostrada no Exemplo 3.36, obtém-se que $\mathcal{C}^{i}$ não é um ultrafiltro em $I$.

Exemplo 3.38. Suponha que $d: I \times I \rightarrow[0, \infty)_{\mathbb{R}}$ é uma pseudométrica em $I$ tal que $I$ é $d$-ilimitado. Mostraremos que o filtro próprio $\mathcal{M}^{d}$ em $I$ (Exemplo 3.8) não é um ultrafiltro. Seja $i$ um elemento de $I$. Como $I$ é ilimitado, existe uma sequência $\left\{i_{n}\right\}_{n \geqslant 1}$ de pontos de $I$ tal que a sequência $\left\{d\left(i, i_{n}\right)\right\}_{n \geqslant 1}$ é crescente e é tal que $d\left(i, i_{n}\right) \rightarrow \infty$ quando $n \rightarrow \infty$. Assim, temos que o conjunto $\left\{i_{2 n}\right\}_{i \geqslant 1}$ é $d$-ilimitado, e o seu complemento em $I$, o qual contém o conjunto $\left\{i_{2 n-1}\right\}_{n \geqslant 1}$, também é $d$-ilimitado, contradizendo o axioma (U') e implicando que $\mathcal{M}^{d}$ não é um ultrafiltro em $I$.

Exemplo 3.39. Seja $\mathcal{V}$ uma $\sigma$-algebra em $I$, e seja $\mu: \mathcal{V} \rightarrow[0, \infty]_{\mathbb{R}}$ uma medida não negativa em $I \operatorname{com} \mu(I)>0$. Se existir um $S \in \mathcal{V}$ tal que $0<\mu(S)<\mu(I)$, então o filtro próprio $\mathcal{O}^{\mu}$ em $I$ (Exemplo 3.9) não será um ultrafiltro em $I$, visto que nesse caso teremos $\mu(I-S)=\mu(I)-\mu(S)>0$, resultando que $S$ e $I-S$ não pertencem a $\mathcal{O}^{\mu}$ e contradizendo o axioma (U').

A partir deste ponto neste capítulo, assumiremos que $\mathcal{U}$ é um ultrafiltro em $I$ e consideraremos que $\mathcal{U}$ é o filtro subentendido no contexto das ocorrências das condições da forma $C(i)$ q.t.p. (Notação 3.14).

Proposição 3.40. Seja $C(i)$ uma condição na variável $i$. A condição $C(i)$ q.t.p. será falsa se, e somente se, $\neg C(i)$ q.t.p. for verdadeira.

Demonstração. A condição $C(i)$ q.t.p. será falsa se, e somente se, tivermos

$$
I-\{i \in I \vdots \neg C(i)\}=\{i \in I \vdots C(i)\} \notin \mathcal{U}
$$

e, pelo axioma (U'), temos que isso é equivalente à condição $\neg C(i)$ q.t.p.

Proposição 3.41. As seguintes condições são equivalentes:
(a) $\mathcal{U}=\mathcal{J}^{i}(\exists i \in I)$ (Exemplo 3.3);
(c) U não é livre;
(b) $\mathcal{U}$ é principal;
(d) $\mathcal{U}$ tem um subconjunto finito de $I$ como elemento.

Demonstração. As implicações $(a) \Rightarrow(b) \Rightarrow(c)$ são imediatas.

$(c) \Rightarrow(d)$ : Como $\mathcal{U}$ não é livre, ele não contém o filtro de Fréchet $\mathcal{C}$ em $I$ (Exemplo 3.6), e existe um $X \subset I$ não nulo tal que $I-X$ é finito e $X \notin \mathcal{U}$, implicando em $I-X \in \mathcal{U}$ por $(\mathrm{U})$. 
$(d) \Rightarrow(a)$ : Sejam $i_{1} \ldots i_{n}$ os elementos de um subconjunto finito de $I$ pertencente a $\mathcal{U}$. Assim, temos

$$
\left\{i_{1}\right\} \cup \cdots \cup\left\{i_{n}\right\} \in \mathcal{U}
$$

e algum $\left\{i_{k}\right\}$ pertence a $\mathcal{U}$ (Teorema 3.33, Letra $(c)$ ), resultando em $\mathcal{U}=\mathcal{J}^{i_{k}}$.

O Axioma da Escolha (Definição B.2) na forma do Lema de Zorn (Teorema B.26) viabiliza uma maneira não construtiva de obter extensões de filtros próprios para ultrafiltros.

Teorema 3.42 (Lema do Ultrafiltro). Todo filtro próprio em I está contido em um ultrafiltro em $I$.

Demonstração. Seja $\mathcal{W}$ o conjunto dos filtros próprios em $I$ que contêm $\mathcal{F}$, o qual é não nulo pois $\mathcal{F} \in \mathcal{W}$. Usaremos o Lema de Zorn para provar que $\mathcal{W}$ tem um elemento maximal quando parcialmente ordenado pela inclusão. Suponha que $\mathcal{K}$ é um subconjunto não nulo de $\mathcal{W}$ que é ordenado pela inclusão. A união $\bigcup \mathcal{K}$ contém $\mathcal{F}$ e não contém o conjunto vazio como elemento. Provaremos que $\bigcup \mathcal{K}$ observa os axiomas (F1) e (F2):

- Se $X \subset Y \subset I$ e $X \in \bigcup \mathcal{K}$, então $X \in A$ para algum $A \in \mathcal{K}$, e teremos $Y \in A$ por (F1), resultando em $Y \in \bigcup \mathcal{K}$ e provando que $\bigcup \mathcal{K}$ satisfaz ao axioma (F1).

- Se $X, Y \in \bigcup \mathcal{K}$, então $X \in A$ e $Y \in B$ para algum $A \in \mathcal{K}$ e algum $B \in \mathcal{K}$. Como $\mathcal{K}$ é ordenado pela inclusão, temos $A \subset B$ ou $B \subset A$, e, sem perda de generalidade, podemos assumir que $B \subset A$ e $X, Y \in A$. Portanto, temos $X \cap Y \in A$ por (F2), e $X \cap Y \in \bigcup \mathcal{K}$, provando que $\bigcup \mathcal{K}$ satisfaz ao axioma (F2).

Assim, $\bigcup \mathcal{K}$ é uma cota superior de $\mathcal{K}$ em $\mathcal{W}$, e, pelo Lema de Zorn, existe um elemento maximal $\mathcal{F}^{\prime}$ no conjunto parcialmente ordenado $\mathcal{W}$. Todo filtro próprio em $I$ que contém $\mathcal{F}^{\prime}$ pertence a $\mathcal{W}$, implicando que $\mathcal{F}^{\prime}$ é um filtro maximal e é um ultrafiltro em $I$ (Teorema 3.33, Letra $(d))$.

Em particular, podemos estender os filtros livres, como o filtro de Fréchet (Exemplo 3.6), para ultrafiltros livres, os quais são não principais.

Corolário 3.43. Existe um ultrafiltro livre em I.

Se $I$ é infinito, então a existência de um ultrafiltro não principal em $I$ é uma condição estritamente mais fraca que o Axioma da Escolha módulo ZF (Definição A.34), e ela não pode ser demonstrada em ZF. De fato, existe um modelo de ZF no qual todo ultrafiltro em qualquer conjunto é principal (42). 
Exemplo 3.44. Se $<$ for uma ordem parcial direcionada pela direita em um subconjunto $J$ de $I$, então, pelo Lema do Ultrafiltro, existirá um ultrafiltro em $I$ que contém $\mathcal{Z}^{J}$ (Exemplo 3.13), o qual terá o conjunto $J$ como elemento. Esse ultrafiltro será livre se $J$ não tiver um maior elemento.

\subsection{Ultraprodutos}

O teorema a seguir foi publicado pela primeira vez por Jerzy Łoś em 1955 (53). Também conhecido por Teorema Fundamental dos Ultraprodutos, ele evidencia a importância dos ultrafiltros e ultraprodutos na Teoria dos Modelos.

Teorema 3.45 (Teorema de Łoś). Seja $\phi\left(x_{1} \ldots x_{n}\right)$ uma L-fórmula (Definição A.14). Para quaisquer $r_{1} \ldots r_{n} \in \prod_{i \in I} M_{i}$, temos

$$
M_{i} \models \phi\left[r_{1}(i) \ldots r_{n}(i)\right] \text { q.t.p. } \Leftrightarrow \prod_{\mathcal{U}} M_{i}=\phi\left[\lim _{M_{i}, \mathcal{U}} r_{1}(i) \ldots \lim _{M_{i}, \mathcal{U}} r_{n}(i)\right] \text {. }
$$

Demonstração. Procederemos por indução sobre a complexidade de $\phi$. Pelas Definições 3.23 e A.34, a validade do resultado é imediata quando $\phi$ for uma fórmula atômica. Suponha que $\phi$ tem complexidade $k$ positiva, e que o teorema já foi provado para fórmulas de complexidade menor que $k$. Assim, temos os seguintes casos:

1. Se $\phi$ for da forma $\left(\neg \phi^{\prime}\left(x_{1} \ldots x_{n}\right)\right)$, então a $L$-fórmula $\phi^{\prime}$ terá complexidade $k-1$, e, pela Proposição 3.40, a negação da condição $M_{i} \models \phi\left[r_{1}(i) \ldots r_{n}(i)\right]$ q.t.p. será equivalente à condição $M_{i} \models \phi^{\prime}\left[r_{1}(i) \ldots r_{n}(i)\right]$ q.t.p., a qual, por sua vez, é equivalente a

$$
\prod_{\mathcal{U}} M_{i} \models \phi^{\prime}\left[\lim _{M_{i}, \mathcal{U}} r_{1}(i) \ldots \lim _{M_{i}, \mathcal{U}} r_{n}(i)\right]
$$

pela hipótese de indução. O resultado desejado segue pela definição da relação de satisfatibilidade.

2. Se $\phi$ for da forma $\left(\phi_{1}\left(x_{1} \ldots x_{n}\right) \wedge \phi_{2}\left(x_{1} \ldots x_{n}\right)\right)$, então a soma das complexidades das $L$-fórmulas $\phi_{1}$ e $\phi_{2}$ será $k-1$, implicando que ambas têm complexidade menor que $k$. Nesse caso, pela letra (c) da Proposição 3.16, a condição $M_{i} \models \phi\left[r_{1}(i) \ldots r_{n}(i)\right]$ q.t.p. será equivalente à conjunção das condições

$$
M_{i}=\phi_{1}\left[r_{1}(i) \ldots r_{n}(i)\right] \text { q.t.p. e } M_{i} \models \phi_{2}\left[r_{1}(i) \ldots r_{n}(i)\right] \text { q.t.p., }
$$

a qual, por sua vez, é equivalente à conjunção das condições

$$
\prod_{\mathcal{U}} M_{i} \models \phi_{1}\left[\lim _{M_{i}, \mathcal{U}} r_{1}(i) \ldots \lim _{M_{i}, \mathcal{U}} r_{n}(i)\right] \text { e } \prod_{\mathcal{U}} M_{i} \models \phi_{2}\left[\lim _{M_{i}, \mathcal{U}} r_{1}(i) \ldots \lim _{M_{i}, \mathcal{U}} r_{n}(i)\right]
$$


pela hipótese de indução. O resultado desejado segue pela definição da relação de satisfatibilidade.

3. Se $\phi$ for da forma $\left(\phi_{1}\left(x_{1} \ldots x_{n}\right) \vee \phi_{2}\left(x_{1} \ldots x_{n}\right)\right)$, então $\phi$ será equivalente à condição

$$
\left[\neg\left[\neg\left(\phi_{1}\left(x_{1} \ldots x_{n}\right)\right) \wedge \neg\left(\phi_{2}\left(x_{1} \ldots x_{n}\right)\right)\right]\right],
$$

e o teorema será válido para $\phi$ pelos casos 1 e 2 .

4. Se $\phi$ for da forma $(\exists y) \chi\left(x_{1} \ldots x_{n}, y\right)$, onde $y$ é uma variável que não está na lista $x_{1} \ldots x_{n},{ }^{5}$ então a $L$-fórmula $\chi$ terá complexidade $k-1$, e, pela letra $(d)$ da Proposição 3.16 , a condição $M_{i}=\phi\left[r_{1}(i) \ldots r_{n}(i)\right]$ q.t.p. será equivalente à condição

$$
\left(\exists s \in \prod_{k \in I} M_{k}\right)\left(M_{i} \models \chi\left[r_{1}(i) \ldots r_{n}(i), s(i)\right] \text { q.t.p. }\right) \text {, }
$$

a qual, por sua vez, é equivalente à condição

$$
\left(\exists s \in \prod_{k \in I} M_{k}\right)\left(\prod_{\mathcal{U}} M_{i} \models \chi\left[\lim _{M_{i}, \mathcal{U}} r_{1}(i) \ldots \lim _{M_{i}, \mathcal{U}} r_{n}(i), \lim _{M_{i}, \mathcal{U}} s(i)\right]\right)
$$

pela hipótese de indução. Como todo elemento de $\prod_{\mathcal{U}} M_{i}$ é uma classe de equivalência de algum elemento do produto $\prod_{k \in I} M_{k}$, temos que o resultado desejado segue pela definição da relação de satisfatibilidade.

5. Se $\phi$ for da forma $(\forall y) \chi\left(x_{1} \ldots x_{n}, y\right)$, onde $y$ é uma variável que não está na lista $x_{1} \ldots x_{n}$, então $\phi$ será equivalente à condição

$$
\left[\neg(\exists y)\left(\neg \chi\left(x_{1} \ldots x_{n}, y\right)\right)\right]
$$

e o teorema será válido para $\phi$ pelos casos 1 e 4 .

Portanto, pelo Princípio da Indução, provamos que o teorema é válido para $L$-fórmulas $\phi$ de qualquer complexidade inteira não negativa.

Veremos que o Teorema de Łoś implica em uma versão fraca do Princípio da Transferência (Corolário 3.71) e obteremos a versão geral desse princípio de forma análoga à que demonstramos o Teorema 3.45 .

$\overline{5}$ Se $y=x_{i}$ para algum $i$, então $x_{i}$ não aparecerá livre em $\phi$, e poderemos escrever

$$
\phi=\phi\left(x_{1} \ldots x_{i-1} x_{i+1} \ldots x_{n}\right) .
$$

Assim, $y$ não estará na lista $x_{1} \ldots x_{i-1} x_{i+1} \ldots x_{n}$, mostrando que basta provarmos o caso em que $y$ não aparece na lista $x_{1} \ldots x_{n}$. 
Corolário 3.46. Toda classe axiomatizável $K$ de L-estruturas (Definição A.37) é fechada sobre ultraprodutos, ou seja, temos $\prod_{\mathcal{U}} M_{i} \in K$ para qualquer família $\left\{M_{i}\right\}_{i \in I}$ de $L$-estruturas em $K$.

Exemplo 3.47. Na Seção 3.8, veremos que é possível obter um corpo não arquimediano como resultado de um ultraproduto de corpos arquimedianos, implicando que a classe dos corpos arquimedianos não é axiomatizável. Isso ocorre devido à limitação do processo de construção das $L_{A O}$-fórmulas (Definição A.14) na lógica de primeira ordem, onde apenas uniões e interseções finitas de $L_{A O}$-fórmulas são permitidas. Em lógicas infinitárias, a Propriedade Arquimediana (Definição 1.20) pode ser expressa pela $L_{A O}$-fórmula infinitária

$$
(\forall x, y)\left(0<x<y \longrightarrow \bigvee_{n=1}^{\infty}(y<\overbrace{x+x+\cdots+x}^{n \text { vezes }})\right)
$$

onde o símbolo $\bigvee_{n=1}^{\infty}$ representa uma disjunção infinita das $L_{A O}$-fórmulas que correspondem a cada número natural $n$. Tal fórmula não pode ser expressa em lógicas finitárias como a que estamos trabalhando nesta dissertação.

Exemplo 3.48. Considere o caso descrito no Exemplo 3.25, e suponha que $\mathcal{F}$ é livre. Assim, temos $\mathcal{C} \subset \mathcal{F}$, e, pelo Lema do Ultrafiltro, podemos assumir que $\mathcal{F} \subset \mathcal{U}$. Para cada número natural $n$, seja $\sigma_{\geqslant n}$ uma $L$-sentença que afirma que uma $L$-estrutura tem

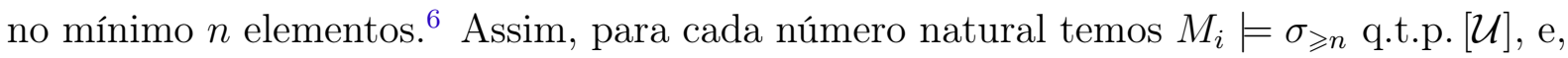
pelo Teorema de Łoś, temos $\prod_{\mathcal{U}} M_{i} \models \sigma_{\geqslant n}(\forall n)$, implicando que o ultraproduto $\prod_{\mathcal{U}} M_{i}$ é infinito. Nota-se que a função $f: \prod_{\mathcal{F}} M_{i} \rightarrow \prod_{\mathcal{U}} M_{i}$ dada por

$$
f\left(\lim _{M_{i}, \mathcal{F}} r(i)\right):=\lim _{M_{i}, \mathcal{U}} r(i)
$$

é bem definida e sobrejetora, resultando que o produto reduzido $\prod_{\mathcal{F}} M_{i}$ é infinito. Ademais, tal função $f$ é um morfismo entre $L$-estruturas.

Exemplo 3.49. Seja $p$ um número primo maior que 2, e, para cada número natural $n$,

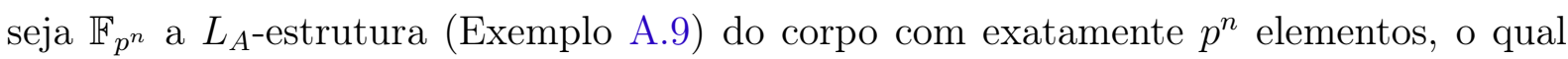
é único salvo isomorfismos e tem característica $p$. Se $n \in \mathbb{N}$ e se todo elemento de $\mathbb{F}_{p^{n}}$

6 A sentença $\sigma_{\geqslant n}$ pode ser escrita na forma

$$
\sigma_{\geqslant n}=\left(\exists x_{1}\right)\left(\exists x_{2}\right) \cdots\left(\exists x_{n}\right) \bigwedge_{1 \leqslant i<j \leqslant n} x_{i} \neq x_{j}
$$

onde $\bigwedge_{1 \leqslant i<j \leqslant n}$ representa uma conjunção finita das $L$-fórmulas que correspondem a cada par $(i, j)$ de números naturais menores ou iguais a $n$ com $i<j$. 
possuir uma raiz quadrada, ou seja, se para todo $x \in \mathbb{F}_{p^{n}}$ existir um $y \in \mathbb{F}_{p^{n}}$ com $x=y^{2}$, então a função $x \mapsto x^{2}$ do tipo $\mathbb{F}_{p^{n}} \rightarrow \mathbb{F}_{p^{n}}$ será sobrejetora, e, como $\mathbb{F}_{p^{n}}$ é finito, tal função será bijetora. Nesse caso, como $(-1)^{2}=1=(1)^{2}$, teremos $-1=1\left(\mathrm{em} \mathbb{F}_{p^{n}}\right)$ e a característica $p$ do corpo $\mathbb{F}_{p^{n}}$ será igual a 2, o que é absurdo. Portanto, para cada $n \in \mathbb{N}$ existe um elemento de $\mathbb{F}_{p^{n}}$ que não tem uma raiz quadrada, e teremos

$$
\mathbb{F}_{p^{n}} \models(\exists x)(\forall y)(x \neq y \cdot y) .
$$

Considerando $I=\mathbb{N}$, pelo Teorema de Łoś temos que o ultraproduto $\prod_{\mathcal{U}} \mathbb{F}_{p^{i}}$ é um corpo infinito (Corolário 3.46 e Exemplo 3.48) de característica $p$ que não é algebricamente fechado. Se $n$ for um número natural, se $I$ for um conjunto infinito de números primos, e se $\mathcal{U}$ for não principal, então o ultraproduto $\prod_{\mathcal{U}} \mathbb{F}_{i^{n}}$ será um corpo infinito de característica 0 que não é algebricamente fechado.

Exemplo 3.50. Seja $\left\{K_{i}\right\}_{i \in I}$ uma família de corpos algebricamente fechados com índices em $I$. Se o ultraproduto $\prod_{\mathcal{U}} K_{i}$ não for algebricamente fechado, então existirá um polinômio em $\prod_{\mathcal{U}} K_{i}$ de grau $k \geqslant 2$ que não possui raízes nesse corpo, e teremos

$$
\prod_{\mathcal{U}} K_{i}=\left(\exists c_{k-1}\right) \cdots\left(\exists c_{0}\right)(\forall x)\left(x^{k}+c_{k-1} \cdot x^{k-1}+\cdots+c_{1} \cdot x+c_{0} \neq 0\right),
$$

onde cada $x^{n}$ é o $L_{A}$-termo

$$
\overbrace{x \cdot x \cdots x}^{n \text { vezes }}
$$

Nesse caso, pelo Teorema de Łoś, teremos

$$
K_{i} \models\left(\exists c_{k-1}\right) \cdots\left(\exists c_{0}\right)(\forall x)\left(x^{k}+c_{k-1} \cdot x^{k-1}+\cdots+c_{1} \cdot x+c_{0} \neq 0\right) \text { q.t.p. }
$$

o que é absurdo visto que cada $K_{i}$ é algebricamente fechado. Portanto, provamos que qualquer ultraproduto de corpos algebricamente fechados é algebricamente fechado.

O Teorema de Łoś implica no corolário a seguir no caso particular da família de $L$-estruturas $\left\{M_{i}\right\}_{i \in I}$ ser constante com $M_{i}=M(\forall i \in I)$.

Corolário 3.51. Seja $M$ uma L-estrutura e seja $d: M \rightarrow \prod_{\mathcal{U}} M$ canônica.

(a) Se $t\left(x_{1} \ldots x_{n}\right)$ for um L-termo e se $a_{1} \ldots a_{n} \in M$, então

$$
d\left(t^{M}\left(a_{1} \ldots a_{n}\right)\right)=t^{\Pi_{\mathcal{U}} M}\left(d\left(a_{1}\right) \ldots d\left(a_{n}\right)\right) ;
$$

(b) Se $\phi\left(x_{1} \ldots x_{n}\right)$ for uma L-fórmula e se $a_{1} \ldots a_{n} \in M$, então

$$
M \models \phi\left[a_{1} \ldots a_{n}\right] \Leftrightarrow \prod_{\mathcal{U}} M \models \phi\left[d\left(a_{1}\right) \ldots d\left(a_{n}\right)\right] .
$$

Em outras palavras, d é uma imersão elementar (Definição A.42); 
(c) $\quad M \equiv \prod_{\mathcal{U}} M($ Definição A.36);

(d) Se $M$ for finita, então as L-estruturas $M e \prod_{\mathcal{U}} M$ serão isomorfas (Definição A.42).

Demonstração. Somente a letra $(d)$ ainda não foi provada.

(d) Assumindo que $M$ é finita, mostraremos que $d$ é sobrejetora, e, portanto, é um isomorfismo pela letra (b). Todo elemento de $\prod_{\mathcal{U}} M$ é da forma $\lim _{M, \mathcal{U}} r(i)$ para $r \in{ }^{I} M=\prod_{i \in I} M$. Seja $r$ uma função desse tipo, sejam $a_{1} \ldots a_{n}$ os elementos de $M$, e, para cada $a_{i}$, seja $J_{i}:=r^{-1}\left\langle\left\{a_{i}\right\}\right\rangle$. Temos

$$
J_{1} \cup J_{2} \cup \cdots \cup J_{n}=I \in \mathcal{U}
$$

e existe um $J_{k}$ pertencente a $\mathcal{U}$ (Teorema 3.33, Letra $(c)$ ), resultando em $r(i)=a_{k}$ q.t.p. e $\lim _{M, \mathcal{U}} r(i)=d\left(a_{k}\right)$.

Ultrafiltros principais não podem ser utilizados para representar $L$-estruturas novas via ultraprodutos, como está evidenciado no corolário a seguir.

Corolário 3.52. Se $\mathcal{U}$ for principal, então existirá um $j \in I$ tal que $\prod_{\mathcal{U}} M_{i} \cong M_{j}$.

Demonstração. Pela Proposição 3.41, existe um $j \in I$ tal que $\mathcal{U}=\mathcal{J}^{j}$, resultando na equivalência

$$
C(i) \text { q.t.p. } \Leftrightarrow C(j)
$$

para qualquer condição matemática $C(i)$ na variável $i$. Assim, nota-se prontamente que a função $f: \prod_{\mathcal{U}} M_{i} \rightarrow M_{j}$ dada por

$$
f\left(\lim _{M_{i}, \mathcal{U}} r(i)\right):=r(j)
$$

é bem definida e é sobrejetora. Pelo Teorema de Łoś, temos

$$
M_{j} \models \phi\left[r_{1}(j) \ldots r_{n}(j)\right] \Leftrightarrow \prod_{\mathcal{U}} M_{i} \models \phi\left[\lim _{M_{i}, \mathcal{U}} r_{1}(i) \ldots \lim _{M_{i}, \mathcal{U}} r_{n}(i)\right]
$$

para quaisquer $r_{1} \ldots r_{n} \in \prod_{i \in I} M_{i}$, e, por conseguinte, $f$ é uma imersão elementar e um isomorfismo. 


\section{7 $\alpha$-Completude de um Filtro}

Pelo axioma (F2), sabemos que interseções finitas de conjuntos em um filtro qualquer pertencem a esse filtro. Porém, o mesmo não é necessariamente válido para interseções infinitas.

Definição 3.53. Seja $\alpha$ um cardinal. Dizemos que um filtro $\mathcal{F}$ sobre $I$ é $\alpha$-completo se ele é fechado sobre interseções com menos de $\alpha$ elementos de $\mathcal{F}$ envolvidos, ou seja, se ele obedecer à seguinte condição:

$\left(\mathbf{F 2}^{\alpha}\right)$ Se $\left\{X_{k}\right\}_{k \in K}$ for uma família de elementos de $\mathcal{F}$ indexada por um conjunto não nulo $K \operatorname{com} K \prec \alpha$ (Definição B.24), então $\bigcap_{k \in K} X_{k} \in \mathcal{F}$.

Caso contrário, dizemos que $\mathcal{F}$ é $\alpha$-incompleto. Dizemos que um filtro é completo se ele é $\alpha$-completo para qualquer cardinal $\alpha$, e caso contrário dizemos que ele é incompleto.

Se $\alpha$ e $\beta$ forem cardinais com $\alpha<\beta$, então todo filtro $\beta$-completo será $\alpha$-completo.

$\mathrm{O}$ axioma $\left(\mathrm{F} 2^{\alpha}\right)$ prontamente implica na condição:

$\left(\right.$ F2 $\left.^{\alpha}{ }^{\prime}\right)$ Se $\left\{X_{\gamma}\right\}_{\gamma<\beta}$ for uma família de elementos de $\mathcal{F}$ indexada por um ordinal não zero $\beta$ menor que $\alpha$, então $\bigcap_{\gamma<\beta} X_{\gamma} \in \mathcal{F}$.

Como estamos admitindo que Axioma da Escolha está em vigor, temos que $\left(\mathrm{F} 2^{\alpha}\right)$ e $\left(\mathrm{F} 2^{\alpha}\right)$ são equivalentes. De fato, se $\left(\mathrm{F} 2^{\alpha}\right)$ for verdadeira, e se $\left\{X_{k}\right\}_{k \in K}$ for uma família de elementos de $\mathcal{F}$ com $K \prec \alpha$, então, pelos Teoremas B.14 e B.26 existirá um ordinal $\beta$ equipotente a $K$ e existirá uma família injetora $\left\{k_{\gamma}\right\}_{\gamma<\beta}$ cujos elementos são os elementos de $K$. Nesse caso, visto que $\alpha$ é um ordinal inicial, teremos $\beta<\alpha$ e

$$
\bigcap_{k \in K} X_{k}=\bigcap_{\gamma<\beta} X_{k_{\gamma}} \in \mathcal{F},
$$

provando que $\left(\mathrm{F}^{\alpha}{ }^{\alpha}\right)$ será verdadeira. Além disso, o Axioma da Escolha nos permite lidar com a concepção do cardinal de um conjunto arbitrário (Definição B.27), e, assim, uma condição da forma $K \prec \alpha$ é equivalente a $|K|<\alpha$.

Exemplo 3.54. O filtro trivial em I (Exemplo 3.2) é completo.

Exemplo 3.55. Pelo axioma (F2), todo filtro finito em $I$ é completo e todo filtro em $I$ é $\omega$-completo. 
Exemplo 3.56. Qualquer interseção de conjuntos em um filtro principal pertence a esse filtro. Assim, todo filtro principal é completo.

Proposição 3.57. As seguintes condições são equivalentes:
(a) $\mathcal{F}$ é não principal;
(c) $\mathcal{F}$ é $|I|^{+}$-incompleto.

(b) $\mathcal{F}$ é $|\mathcal{F}|^{+}$-incompleto;

Demonstração. As provas das condições contrapositivas das implicações $(b) \Rightarrow(a)$ e $(c) \Rightarrow(a)$ são imediatas (Exemplo 3.56).

$(a) \Rightarrow(b)$ : Se $\mathcal{F}$ for $|\mathcal{F}|^{+}$-completo, então $\{X\}_{X \in \mathcal{F}}$ será uma família de elementos de $\mathcal{F}$ indexada pelo conjunto não nulo $\mathcal{F}$ com cardinal menor que $|\mathcal{F}|^{+}$, e, por $\left(\mathrm{F} 2^{\alpha}\right)$, teremos

$$
\bigcap \mathcal{F}=\bigcap_{X \in \mathcal{F}} X \in \mathcal{F}
$$

implicando que o filtro $\mathcal{F}$ será principal.

$(a) \Rightarrow(c)$ : Suponha que $\mathcal{F}$ é $|I|^{+}$-completo, e seja $\mathcal{S}$ o conjunto

$$
\mathcal{S}:=\{I-\{i\} \vdots i \in I \text { e } I-\{i\} \in \mathcal{F}\} \subset \mathcal{F}
$$

Temos $|\mathcal{S}| \leqslant|I|<|I|^{+}$e $\bigcap \mathcal{S} \in \mathcal{F}$ por $\left(\mathrm{F} 2^{\alpha}\right)$. Se $X \in \mathcal{F}$ e $i \in I-X$, então teremos $X \subset I-\{i\}, I-\{i\} \in \mathcal{F}$ por (F1), e $i \notin I-\{i\} \in \mathcal{S}$, resultando em $i \notin \bigcap \mathcal{S}$. Portanto, temos $\bigcap \mathcal{S} \subset X(\forall X \in \mathcal{F})$, e, por $(\mathrm{F} 1)$, provamos que $\mathcal{F}$ é o filtro principal dos subconjuntos de $I$ que contêm $\bigcap \mathcal{S}$.

Em particular, todo filtro não principal em $\mathbb{N}$ é $\omega_{1}$-incompleto.

Valendo-se do Axioma da Escolha, pode-se atestar a existência de um filtro $\omega_{1}$-incompleto em qualquer conjunto infinito. A demonstração desse resultado está fora do escorpo desta dissertação e pode ser encontrada em (13).

Teorema 3.58. Se I for infinito, então existirá um ultrafiltro $\omega_{1}$-incompleto em $I$.

Se $P_{1} P_{2} \ldots P_{n}$ for uma partição finita de $I$, ou seja, se $P_{1} P_{2} \ldots P_{n}$ forem subconjuntos disjuntos de $I$ que cobrem $I$, então teremos

$$
P_{1} \cup P_{2} \cup \cdots \cup P_{n}=I \in \mathcal{U},
$$

e, pela letra $(c)$ do Teorema 3.33, pelo menos um $P_{i}$ pertencerá a $\mathcal{U}$. A proposição a seguir assegura que esse fato pode ser estendido para partições infinitas de $I$ quando o ultrafiltro $\mathcal{U}$ for $\alpha$-completo $\operatorname{com} \omega<\alpha$. 
Proposição 3.59. Seja $\alpha$ um cardinal infinito. $O$ ultrafiltro $\mathcal{U}$ será $\alpha$-completo se, e somente se, toda partição de I em uma quantidade de partes menor que a tiver pelo menos uma dessas partes pertencente a $\mathcal{U}$.

Demonstração. Se $\mathcal{U}$ for $\alpha$-completo, e se $\left\{P_{k}\right\}_{k \in K}$ for uma partição ${ }^{7}$ de $I$ com $|K|<\alpha$, então teremos

$$
\bigcap_{k \in K}\left(I-P_{k}\right)=\emptyset \notin \mathcal{U}
$$

e, por $\left(\mathrm{F} 2^{\alpha}\right)$, existirá um $k \in K$ com $I-P_{k} \notin \mathcal{U}$, implicando em $P_{k} \in \mathcal{U}$ por (U').

Suponha que toda partição de $I$ em uma quantidade de partes menor que $\alpha$ tem pelo menos uma dessas partes pertencente a $\mathcal{U}$, e seja $\left\{X_{\gamma}\right\}_{\gamma<\beta}$ uma família de elementos de $\mathcal{U}$ indexada por um ordinal não zero $\beta$ menor que $\alpha$. Provaremos que $\bigcap_{\gamma<\beta} X_{\gamma} \in \mathcal{U}$. Seja $f: I \rightarrow \beta^{\prime}$ (Definição B.15) a função definida da seguinte maneira:

- Se $i \in \bigcap_{\gamma<\beta} X_{\gamma}$, então $f(i):=\beta$;

- Se $i \in I-\bigcap_{\gamma<\beta} X_{\gamma}$, então $f(i)$ será o menor ordinal $\gamma$ menor que $\beta$ tal que $i \notin X_{\gamma}$.

Note que teremos $f(i)=\beta$ se, e somente se, tivermos $i \in \bigcap_{\gamma<\beta} X_{\gamma}$. O conjunto $\left\{f^{-1}\langle\{\gamma\}\rangle\right\}_{\gamma \leqslant \beta}$ é uma partição de $I$ em uma quantidade de partes menor que $\alpha$, e, pela suposição, existe um ordinal $\eta \leqslant \beta$ tal que $f^{-1}\langle\{\eta\}\rangle \in \mathcal{U}$. Se $\eta<\beta$, e se $i$ for um elemento de $f^{-1}\langle\{\eta\}\rangle$, então $i \notin X_{\eta}$ pela definição de $f$, implicando em $f^{-1}\langle\{\eta\}\rangle \cap X_{\eta}=\emptyset$, o que é absurdo por (F2). Assim, $\eta=\beta$, e temos

$$
\bigcap_{\gamma<\beta} X_{\gamma}=f^{-1}\langle\{\beta\}\rangle \in \mathcal{U}
$$

provando que $\mathcal{U}$ é $\alpha$-completo por $\left(\mathrm{F} 2^{\alpha}{ }^{\prime}\right)$.

Dada uma $L$-estrutura $M$, é claro que a imersão canônica $d: M \rightarrow \prod_{\mathcal{U}} M$ é injetora. No entanto, a situação da sobrejetividade dessa função depende da $L$-estrutura $M$ e do ultrafiltro $\mathcal{U}$, e, caso $M$ seja infinita, esse quadro reduz-se à condição de completude do ultrafiltro $\mathcal{U}$, como mostra a proposição a seguir.

Proposição 3.60. Seja $M$ uma L-estrutura infinita. A imersão canônica d: $M \rightarrow \prod_{\mathcal{U}} M$ será sobrejetora se, e somente se, o ultrafiltro $\mathcal{U}$ for $|M|^{+}$-completo.

7 Uma partição de $I$ é uma família $\left\{P_{k}\right\}_{k \in K}$ de subconjuntos não nulos de $I$ tal que $I=\bigcup_{k \in K} P_{k}$ e $P_{k} \cap P_{l}=\emptyset(\forall k, l \in K)$. 
Demonstração. Se $\mathcal{U}$ for $|M|^{+}$-completo, e se $r: I \rightarrow M$ for uma função, então o conjunto $\left\{r^{-1}\langle\{a\}\rangle\right\}_{a \in \operatorname{Im}(r)}$ será uma partição de $I$ em uma quantidade de partes menor que $|M|^{+}$, e, pela Proposição 3.59, existirá um elemento $a \operatorname{de} \operatorname{Im}(r) \operatorname{com} r^{-1}\langle\{a\}\rangle \in \mathcal{U}$, resultando em $d(a)=\lim _{M, \mathcal{U}} r(i)$, e provando que a função $d$ é sobrejetora.

Suponha que $d$ é sobrejetora, e seja $\left\{P_{k}\right\}_{k \in K}$ uma partição de $I$ com $|K|<|M|^{+}$. Assim, temos $|K| \leqslant|M|$ e existe uma injeção do tipo $K \rightarrow M$, de modo que podemos assumir que $K$ é um subconjunto de $M$. Seja $f: I \rightarrow M$ a função definida por

$$
f(i):=k \text { se } i \in P_{k} \text {. }
$$

Como $d$ é sobrejetora, existe um $a \in M$ com

$$
d(a)=\lim _{M, \mathcal{U}} a=\lim _{M, \mathcal{U}} f(i)
$$

resultando em $f^{-1}\langle\{a\}\rangle \in \mathcal{U}, f^{-1}\langle\{a\}\rangle \neq \emptyset, a \in \operatorname{Im}(f)=K, \mathrm{e}$

$$
P_{a}=f^{-1}\langle\{a\}\rangle \in \mathcal{U}
$$

Portanto, pela Proposição 3.59, o ultrafiltro $\mathcal{U}$ é $|M|^{+}$-completo.

\subsection{A Ultrapotência * $\mathbb{R}$}

Nesta seção, assumiremos que $I=\mathbb{N}$ e que o ultrafiltro $\mathcal{U}$ em $\mathbb{N}$ é não principal. Como $\mathcal{C}$ é livre, o Lema do Ultrafiltro implica que ultrafiltros desse tipo existem em $\mathbb{N}$. Também assumiremos que o conjunto dos números reais, $\mathbb{R}$, está munido de sua $L_{A O}$-estrutura usual de corpo ordenado.

Definição 3.61. A ultrapotência $\prod_{\mathcal{U}} \mathbb{R}$ é dita ser o corpo ordenado dos números hiper-reais, e ela é denotada por ${ }^{*} \mathbb{R}$. Pelo Corolário 3.46, tal estrutura é um corpo ordenado que estende $\mathbb{R}$. Assim como fizemos na Seção 1.10, identificaremos os elementos da imagem da imersão canônica $d: \mathbb{R} \rightarrow{ }^{*} \mathbb{R}$ com os números reais, e, por abuso de linguagem, escreveremos $\mathbb{R} \subset{ }^{*} \mathbb{R}$. Além disso, denotaremos a adição, a multiplicação e a ordem em * $\mathbb{R}$ por,$+ \cdot$ e $<$, respectivamente.

Notação 3.62 (Limites Generalizados em * ${ }^{*}$ ).

- Se $A$ é um subconjunto de $\mathbb{R}$, então o ultralimite integral $\lim _{\mathbb{R}, \mathcal{U}} A$ é denotado por ${ }^{*} A$ e é chamado de $*$-transformação de $A$; 
- Se $A^{1} A^{2} \ldots A^{n}$ são subconjuntos de $\mathbb{R}$, e se $R \subset A^{1} \times A^{2} \times \cdots \times A^{n}$ é uma relação $n$-ária, então ultralimite relacional

$$
\lim _{\mathbb{R}, \mathcal{U}} R \subset^{*} A^{1} \times{ }^{*} A^{2} \times \cdots \times{ }^{*} A^{n}
$$

é denotado por ${ }^{*} R$ e é chamado de $*$-transformação de $R$.

Exemplo 3.63. A adição, a multiplicação e a ordem na ultrapotência * $\mathbb{R}$ são respectivamente iguais aos ultralimites relacionais da adição, da multiplicação e da ordem em $\mathbb{R}$ (Seção 3.4).

Exemplo 3.64. A *-transformação do conjunto vazio, * $\emptyset$, é igual ao conjunto vazio (Exemplo 3.28).

Pela Proposição 3.29, os limites integrais ${ }^{*} \mathbb{N},{ }^{*} \mathbb{Z},{ }^{*} \mathbb{Q}$ e ${ }^{*} \operatorname{Irr}$ são cópias das respectivas ultrapotências $\prod_{\mathcal{U}} \mathbb{N}, \prod_{\mathcal{U}} \mathbb{Z}, \prod_{\mathcal{U}} \mathbb{Q}$ e $\prod_{\mathcal{U}} I r r$, e, assim como fizemos no Capítulo 2, chamaremos os elementos desses conjuntos de números hipernaturais, números hiperinteiros, números hiper-racionais e números hiperirracionais, respectivamente. Nota-se que as ultrapotências mencionadas têm os mesmos traços estruturais dos respectivos limites integrais que elas espelham em ${ }^{*} \mathbb{R}$.

Exemplo 3.65. Seja $\left\{q_{n}\right\}$ a sequência definida na Seção 1.9, e considere o número hiperreal $w$ dado pelo ultralimite $w:=\lim _{\mathbb{R}, \mathcal{U}} q_{i}$. Claramente, temos $q_{i} \in \mathbb{Q}(\forall i)$, e, pela definição dos limites integrais, temos $w \in{ }^{*} \mathbb{Q}$. Assim, $w$ é um número hiper-racional mesmo sendo gerado por uma sequência cujo limite em $\mathbb{R}$ é um número irracional.

Exemplo 3.66. Considere o número hiper-real $h$ dado pelo ultralimite $h:=\lim _{\mathbb{R}, \mathcal{U}} 1 / i$. Como $0<1 / i(\forall i)$, temos $0<\lim _{\mathbb{R}, \mathcal{U}} 1 / i=h$, e se $n \in \mathbb{N}$, então teremos

$$
\begin{aligned}
n h & =\overbrace{h+\cdots+h}^{n \text { vezes }} \\
& =\lim _{\mathbb{R}, \mathcal{U}} \frac{\overbrace{1+\cdots+1}^{n \text { vezes }}}{i} \\
& =\lim _{\mathbb{R}, \mathcal{U}} \frac{n}{i} \\
& <1,
\end{aligned}
$$

visto que $(n, \infty)_{\mathbb{N}} \in \mathcal{C} \subset \mathcal{U}$. Portanto, $h$ é um infinitesimal positivo em ${ }^{*} \mathbb{R}$, e $h^{-1}$ é infinito (Teorema 1.18). Analogamente, nota-se que o ultralimite $\lim _{\mathbb{R}, \mathcal{U}} \sqrt{2} / i$ é um número hiperirracional positivo e infinitesimal. Provamos, assim, o teorema a seguir.

Teorema 3.67. O corpo ordenado dos números hiper-reais é não arquimediano. 
Exemplo 3.68. Considere o número hiper-real $u$ dado pelo ultralimite $u:=\lim _{\mathbb{R}, \mathcal{U}}(-1)^{i}$. Pelo Axioma (U') (Seção 3.5), temos que ou $(-1)^{i}=1$ q.t.p. ou $(-1)^{i}=-1$ q.t.p., resultando em ou $u=1$ ou $u=-1$. Como os conjuntos ordenados $2 \mathbb{N}$ e $2 \mathbb{N}-1$ são direcionados pela direita e não possuem um maior elemento, é possível escolher o ultrafiltro não principal $\mathcal{U}$ em $\mathbb{N}$ de modo que cada uma dessas duas alternativas vigora (Exemplo 3.44).

Exemplo 3.69. Considere a sequência $\{\cos (i)\}_{i \geqslant 1}$, onde os ângulos nos argumentos da função cosseno são dados em radianos. Se $m$ e $n$ forem números naturais com $\cos (m)=\cos (n)$, então existirá um número inteiro $k$ com $m \pm n=2 \pi k$. Nesse caso, se $k \neq 0$, então o número $\pi$ será racional, o que é absurdo, implicando que $k=0$ e $m=n$. Assim, a sequência $\{\cos (i)\}_{i \geqslant 1}$ é injetora, e, como o ultrafiltro $\mathcal{U}$ em $\mathbb{N}$ é não principal, temos que o ultralimite $\lim _{\mathbb{R}, \mathcal{U}} \cos (i)$ não é um número real em $* \mathbb{R}$.

Como $\pi$ é um número irracional, temos $\cos (i) \neq \pm 1(\forall i)$. Provaremos que o conjunto $\{\cos (i)\}_{i \geqslant 1}$ é denso em $[-1,1]_{\mathbb{R}}$. Sejam $a, b \in[-1,1]_{\mathbb{R}} \operatorname{com} a<b$, e seja $u$ um número real com $\cos (u)=(a+b) / 2$. Como a função cosseno é contínua, teremos que existe um número real positivo $h$ tal que

$$
|x-u|<h \Rightarrow\left|\cos (x)-\frac{a+b}{2}\right|<\frac{b-a}{2}
$$

para todo número real $x$, e, como o conjunto $\mathbb{Z}+2 \pi \mathbb{Z}$ é denso em $\mathbb{R}$ (Exemplo 1.5), existem números inteiros $n$ e $k$ tais que $|n+2 \pi k-u|<h$ e

$$
\left|\cos (|n|)-\frac{a+b}{2}\right|=\left|\cos (n+2 \pi k)-\frac{a+b}{2}\right|<\frac{b-a}{2},
$$

resultando em $a<\cos (|n|)<b$, e provando que $\{\cos (i)\}_{i \geqslant 1}$ é denso em $[-1,1]_{\mathbb{R}}$.

Seja $r \in[-1,1]_{\mathbb{R}}$ qualquer, seja $\left\{n_{i}\right\}_{i \geqslant 1}$ uma sequência injetora de números naturais tal que $\left|\cos \left(n_{i}\right)-r\right|<1 / i$ para cada índice $i \in \mathbb{N}$, e considere que $\mathcal{U}$ é um ultrafiltro não principal em $\mathbb{N}$ que contém o filtro $\mathcal{Z}^{\left\{n_{i}\right\}_{i \geqslant 1}}$ (Exemplo 3.44). Para cada número natural positivo $k$, temos

$$
-\frac{1}{k}<\lim _{\mathbb{R}, \mathcal{U}}(\cos (i)-r)<\frac{1}{k}
$$

implicando em $\lim _{\mathbb{R}, \mathcal{U}} \cos (i) \sim r$. Portanto, para cada $r \in[-1,1]_{\mathbb{R}}$ existe um ultrafiltro não principal $\mathcal{U}$ em $\mathbb{N}$ tal que o número hiper-real $\lim _{\mathbb{R}, \mathcal{U}} \cos (i)$ é infinitamente próximo a $r$.

O corpo ordenado dos números hiper-reais satisfaz à letra (e) do Teorema 2.3 para subconjuntos de $\mathbb{R}$ :

Corolário 3.70. Se $A$ for um subconjunto de $\mathbb{R}$, então $A \subset{ }^{*} A$, e se além disso $A$ for infinito, então $A \subsetneq^{*} A$. 
Demonstração. A prova da inclusão $A \subset{ }^{*} A$ é imediata. Suponha que $A$ é infinito. Assim, temos $\omega_{1} \leqslant|A|^{+}$, e, como $\mathcal{U}$ é $\omega_{1}$-incompleto (Proposição 3.57), o ultrafiltro $\mathcal{U}$ é $|A|^{+}$-incompleto, resultando que a imersão canônica $e: A \rightarrow \prod_{\mathcal{U}} A$ não é sobrejetora (Proposição 3.60). Seja $u: \prod_{\mathcal{U}} A \rightarrow{ }^{*} A$ a bijeção canônica (Proposição 3.29). Portanto, a composição $u \circ e: A \rightarrow{ }^{*} A$ não é sobrejetora, e, como $(u \circ e)(x)=d(x)(\forall x \in A)$, existe um elemento de ${ }^{*} A$ que não é da forma $d(x)$ para $x \in A$.

O Teorema de Łoś (Teorema 3.45) implica na seguinte variante do Princípio da Transferência:

Corolário 3.71 (Princípio da Transferência; PT - Versão para $\prod_{\mathcal{U}} \mathbb{R}$ ). Para toda

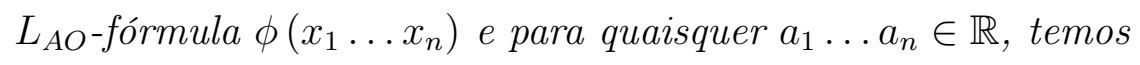

$$
\mathbb{R} \models \phi\left[a_{1} \ldots a_{n}\right] \Leftrightarrow{ }^{*} \mathbb{R} \models \phi\left[a_{1} \ldots a_{n}\right]
$$

ou seja, ${ }^{*} \mathbb{R}$ é uma extensão elementar de $\mathbb{R}$. Em particular, temos

$$
\mathbb{R} \models \phi \Leftrightarrow{ }^{*} \mathbb{R} \models \phi
$$

para toda $L_{A O-s e n t e n c ̧ a ~} \phi$, ou seja, os corpos ordenados $\mathbb{R} \quad e \quad{ }^{*} \mathbb{R}$ são elementarmente equivalentes.

A versão informal do Princípio da Transferência com a qual operamos no Capítulo 2 é mais abrangente e aplicável que a do Corolário 3.71. Essa disparidade dá-se, primeiramente, pelo fato de que nesta seção definimos apenas as $*$-transformações dos números reais, dos conjuntos de números reais e das relações $n$-árias em $\mathbb{R}$, deixando indeterminada a concepção da $*$-transformação de um $\mathbb{R}$-objeto qualquer, a qual foi postulada no Teorema 2.3. Ademais, na Seção 2.3 permitimos que o símbolo da relação de pertinência, $\epsilon$, aparecesse nas condições matemáticas, e isso nos possibilitou trabalhar com condições matemáticas mais expressivas que as consideradas na lógica de primeira ordem, como, por exemplo, as sentenças de segunda ordem. ${ }^{8}$

8 Embora as condições matemáticas especificadas na Seção 2.3 sejam fórmulas de primeira ordem, algumas delas têm aspecto de fórmulas de segunda ordem. A título de exemplo, na sentença verdadeira

$$
(\forall S \in \mathcal{P}(\mathbb{R}))(\forall x \in \mathbb{R})(x \in S \vee x \notin S)
$$

há uma quantificação sobre os subconjuntos de $\mathbb{R}$ típica das sentenças da lógica de segunda ordem. 


\section{4 \\ Monomorfismos Não Standard}

No Capítulo 2, trabalhamos informalmente com a concepção de um monomorfismo não standard * (Teorema 2.3), uma função que origina uma conexão lógica entre dois terrenos matemáticos: os objetos relacionados a $\mathbb{R}$ e os objetos relacionados a * $\mathbb{R}$. Veremos neste capítulo que essa ideia pode ser adequadamente formalizada mediante os conceitos da Teoria dos Modelos (Apêndice A), e ela não é aplicável apenas à extensão do conjunto dos números reais para o conjunto dos números hiper-reais: ela pode ser utilizada para conectar o universo relacionado a um conjunto $X$ qualquer ao universo relacionado a um conjunto ${ }^{*} X$ associado. Dessa forma, os resultados do Capítulo 2 correspondem ao caso especial $X=\mathbb{R}$, e outras escolhas de $X$ são proveitosas em diversas outras áreas da Matemática como a Topologia, a Teoria das Probabilidades, a Análise Funcional, a Teoria das Medidas, etc.

\section{$4.1 \quad X_{\omega}$-Objetos}

Vimos no Capítulo 2 que a classe dos objetos relacionados a $\mathbb{R}($ ou $* \mathbb{R})$ é igual ao universo de von Neumann, $\mathrm{V}$, tornando a definição desses objetos supérflua e infrutífera. Nesta seção, desenvolveremos uma noção similar à que foi elaborada na Seção 2.2, a qual confina os objetos definidos em uma classe não própria (i.e., um conjunto). O leitor é encorajado a comparar as definições e os resultados apresentados nesta parte com os da Seção B.5.

Pelo restante desta dissertação, assumiremos que $X$ é um conjunto qualquer.

Definição 4.1. Denotaremos por

$$
X_{0} X_{1} \ldots X_{n} \ldots X_{\omega}
$$

a sequência de conjuntos definida recursivamente (Teorema B.21) da seguinte maneira:

$$
\text { - } X_{0}:=X ; \quad \bullet X_{\omega}:=\bigcup_{n<\omega} X_{n}{ }^{1}
$$

- $X_{n+1}:=X_{n} \cup \mathcal{P}\left(X_{n}\right)(\forall n<\omega)$;

Os índices dessa sequência são os ordinais finitos e o ordinal infinito $\omega$ (Seção B.3). Temos as seguintes terminologias e notações:

1 O Teorema B.21 permite que a sequência dos $X_{\alpha}$ seja estendida para qualquer índice $\alpha$ em On. Para os propósitos da Análise Não Standard, será suficiente lidar apenas com a parte dessa sequência cujos índices são menores ou iguais a $\omega$. 
- Os elementos de $X_{\omega}$ são chamados de $X_{\omega}$-objetos;

- Cada conjunto $X_{n}$ com $n \leqslant \omega$ é dito ser o $n$-ésimo nível de $X_{\omega}$, e dizemos que cada elemento de $X_{n}$ tem nível $n$ em $X_{\omega}$;

- Os elementos de $X$, os quais são os $X_{\omega}$-objetos de nível 0, são chamados de átomos em $X_{\omega}$ ou $X_{\omega}$-átomos;

- Cada diferença $X_{n}-X$ com $n \leqslant \omega$ é denotada por $^{2} X^{n}$ e é dita ser o $n$-ésimo nível de $X^{\omega}$. Os elementos de $X^{\omega}$ são chamados de conjuntos em $X_{\omega}$ ou $X_{\omega}$-conjuntos, e dizemos que cada elemento de $X^{n}$ tem nível $n$ em $X^{\omega}$.

As sequências $\left\{X_{n}\right\}$ e $\left\{X^{n}\right\}$ são crescentes em relação à inclusão, e temos $X^{0}=\emptyset$, $X^{n} \subset X_{n}(\forall n \leqslant \omega)$, e $X^{\omega} \notin X_{\omega}$. O Teorema B.21 assegura que as classes $X_{n}$ com $n \leqslant \omega$ não são próprias.

A recursividade da Definição 4.1 nos permitirá construir o monomorfismo não standard $*$ em níveis

$$
*_{0} \subset *_{1} \subset *_{2} \subset \ldots
$$

de modo que cada função $*_{n}$ será do tipo $X_{n} \rightarrow Y_{n}$ e $*$ será a extensão das funções dessa sequência (Seção 5.2).

Exemplo 4.2. Prova-se facilmente por indução sobre $n \geqslant 1$ que se o conjunto $X$ for finito, então todo $X_{\omega}$-conjunto de nível $n$ será finito, implicando que todo $X_{\omega}$-conjunto será finito.

Exemplo 4.3. Nota-se que as sequências $\emptyset_{0} \emptyset_{1} \ldots \emptyset_{\omega}$ e $V_{0} V_{1} \ldots V_{\omega}$ são definidas da mesma maneira (Seção B.5), resultando em $\emptyset_{n}=\mathrm{V}_{n}(\forall n \leqslant \omega)$. Para qualquer conjunto $X$ temos $\emptyset \subset X$ e $\emptyset_{n} \subset X_{n}(\forall n \leqslant \omega)$.

O conjunto vazio, $\emptyset$, pertence ao nível $X_{1}$ em $X_{\omega}$, mas ele também pode ser um $X_{\omega}$-átomo. Também pode ocorrer de algum $X_{\omega}$-átomo ter elementos que pertencem ao conjunto $X_{\omega}$. Nesta seção, veremos que esses casos são indesejáveis e inconvenientes, e, por conseguinte, nos ateremos a trabalhar com conjuntos $X$ que não têm tais especificidades. Na Seção 5.1, uma maneira de superar essa limitação será apresentada, de modo que poderemos lidar com qualquer conjunto $X$ (ou pelo menos com uma cópia dele) na construção do monomorfismo não standard $*: X_{\omega} \rightarrow Y_{\omega}$.

2 Embora a notação para o $n$-ésimo nível de $X^{\omega}$ seja idêntica à notação para o produto cartesiano

$$
\overbrace{X \times X \times \cdots \times X}^{n \text { vezes }}
$$

o autor providenciará contextos suficientemente claros para que o leitor tenha ciência de qual objeto matemático a notação $X^{n}$ representa. 
Definição 4.4. Dizemos que um conjunto $X$ é um conjunto base se $\emptyset \notin X$ e

$$
x \cap X_{\omega}=\emptyset(\forall x \in X) .
$$

Se $X$ for um conjunto base, então o conjunto vazio será um $X_{\omega^{-}}$conjunto, e todo $X_{\omega}$-objeto ou será um $X_{\omega}$-átomo ou um $X_{\omega}$-conjunto.

Exemplo 4.5. O conjunto vazio, $\emptyset$, é um conjunto base.

Exemplo 4.6. Se $x \cap \emptyset_{\omega} \neq \emptyset$ para algum $x \in X$, então $X$ não será um conjunto base, visto que $\emptyset_{\omega} \subset X_{\omega}$ (Exemplo 4.3).

As condições que determinam que um conjunto $X$ é um conjunto base são regularmente utilizadas para demonstrar que alguns $X_{\omega}$-objetos não são $X_{\omega}$-átomos. A título

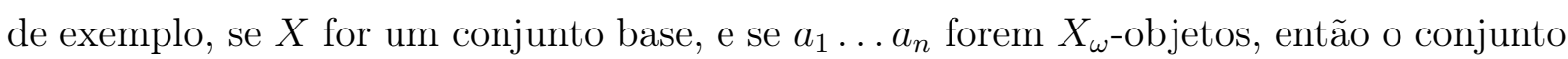
$\left\{a_{1} \ldots a_{n}\right\}$ não será um $X_{\omega}$-átomo, visto que

$$
\left\{a_{1} \ldots a_{n}\right\} \cap X_{\omega}=\left\{a_{1} \ldots a_{n}\right\} \neq \emptyset \text {. }
$$

Prova-se diretamente por indução sobre $n$ que a $n$-tupla $\left(a_{1} \ldots a_{n}\right)$ é um $X_{\omega}$-conjunto.

Até o final deste capítulo, admitiremos que $X$ é um conjunto base.

Proposição 4.7. Para cada número natural $n$, temos $X^{n}=\mathcal{P}\left(X_{n-1}\right)$.

Demonstração. Temos $\mathcal{P}\left(X_{n-1}\right) \subset X_{n}$, e se $x \in X \cap \mathcal{P}\left(X_{n-1}\right)$, então, como $X$ é um conjunto base, teremos $x \neq \emptyset$, e existirá um $y \in x \subset X_{n-1}$, resultando em $y \in X_{\omega}$, o que é absurdo visto que $x \cap X_{\omega}=\emptyset$. Portanto, provamos que $\mathcal{P}\left(X_{n-1}\right) \subset X^{n}$, e provaremos a inclusão oposta por indução sobre $n$. O resultado é imediato para $n=1$. Suponha que ele é verdadeiro para um número natural $n$, e tome $A \in X^{n+1}$. Temos $A \in X^{n}$ ou $A \in \mathcal{P}\left(X_{n}\right)$. No primeiro caso, a hipótese de indução implica em $A \subset X_{n-1} \subset X_{n}$. Assim, em ambos os casos temos $A \in \mathcal{P}\left(X_{n}\right)$, finalizando a indução.

Temos $X_{n}=X \cup \mathcal{P}\left(X_{n-1}\right)$ para cada número natural $n$, onde tal união é disjunta. Para cada número natural $n$, as condições $A \in X^{n}$ e $A \subset X_{n-1}$ são equivalentes, e se todo elemento de $A$ for um $X_{\omega}$-conjunto, então as condições $A \in X^{n}$ e $A \subset X^{n-1}$ serão equivalentes.

Teorema 4.8 (Propriedades de $\left.X_{\omega}\right)$. Seja $n<\omega$. Temos:

(a) Se $A \in X^{n}$ então $\mathcal{P}(A) \in X^{n+1}$;

(b) $\quad S e S \subset A \in X^{n}$, então $S \in X^{n}$;

(c) $\quad$ Se $\emptyset \neq \mathcal{A} \subset X^{\omega}$, ent $\tilde{a} o \bigcap \mathcal{A} \in X^{\omega}$; (d) $S e \mathcal{A}$ for um $X_{\omega}$-conjunto de nivel $n$ cujos elementos são $X_{\omega}$-conjuntos, então $\bigcup \mathcal{A} \in X^{n-1}$ 
(e) Se $A, B \in X^{n}$, então $A-B \in X^{n}$;

(f) Se $A, B \in X^{n}$, então $A \times B \in X^{n+2}$;

Seja $a_{1} a_{2} \ldots a_{m}$ uma sequência finita de $X_{\omega}$-objetos com $m \geqslant 2$.

(g) Teremos $a_{i} \in X_{n}(\forall i)$ se, e somente se, $\left\{a_{1} a_{2} \ldots a_{m}\right\} \in X^{n+1}$;

(h) Teremos $a_{1}, a_{2} \in X_{n}$ e $a_{i} \in X_{n+2 i-4}(\forall i \geqslant 3)$ se, e somente se, a m-tupla $\left(a_{1} \ldots a_{m}\right)$ pertencer a $X^{n+2 m-2}$;

(i) Se $A, B \in X^{n}$, se $R \subset A \times B$, e se $A \subset \operatorname{dom}(R)$, então $R, R^{-1} \in X^{n+2}$ e $R\langle A\rangle \in X^{n}$. Em particular, toda função do tipo $A \rightarrow B$ será um $X_{\omega}$-conjunto de nível $n+2$;

(j) Se $R$ for uma relação binária em $X_{\omega} \operatorname{com} R \in X^{n}$, e se $A \subset \operatorname{dom}(R)$, então o conjunto $R$ será uma relação binária em $X_{n-3}$, e as condições $R^{-1} \in X^{n} e$ $A, R\langle A\rangle \in X^{n-2}$ serão verdadeiras;

(k) Se $R$ e $S$ forem relações binárias em $X_{n}$, então $R \circ S \in X^{n+3}$;

(1) Se $\left\{A_{i}\right\}_{i \in I}$ for uma família de $X_{\omega}$-conjuntos pertencente a $X^{n}$ com $I \subset X_{\omega}$, então $\prod_{i \in I} A_{i} \in X^{n+1}$.

Demonstração.

(a) Temos $A \subset X_{n-1}$, e todo subconjunto de $A$ é um subconjunto de $X_{n-1}$, resultando $\operatorname{em} \mathcal{P}(A) \subset \mathcal{P}\left(X_{n-1}\right) \subset X_{n}$ e $\mathcal{P}(A) \in X^{n+1}$.

(b) Temos $S \subset A \subset X_{n-1}$ e $S \in X^{n}$.

(c) Se $A \in \mathcal{A}$ com $A \in X^{m}(m<\omega)$, então teremos $\bigcap \mathcal{A} \subset A \subset X_{m-1}$ e $\bigcap \mathcal{A} \in X^{m}$.

(d) Temos $\mathcal{A} \subset X^{n-1}$ e $A \subset X_{n-2}(\forall A \in \mathcal{A})$, resultando em $\bigcup \mathcal{A} \subset X_{n-2}$ e $\bigcup \mathcal{A} \in X^{n-1}$.

(e) Temos $A-B \subset A \subset X_{n-1}$, implicando em $A-B \in X^{n}$.

(f) Como $A \times B \subset \mathcal{P}(\mathcal{P}(A \cup B))$, o resultado segue pelas letras $(a)$ e $(d)$.

(g) Basta observar que as condições $\left\{a_{1} a_{2} \ldots a_{m}\right\} \in X^{n+1}$ e $\left\{a_{1} a_{2} \ldots a_{m}\right\} \subset X_{n}$ são equivalentes.

(h) Provaremos o resultado desejado por indução sobre $m$. Se $a_{1}, a_{2} \in X_{n}$, então os conjuntos $\left\{a_{1}\right\}$ e $\left\{a_{1}, a_{2}\right\}$ pertencerão a $X^{n+1}$, e teremos

$$
\left(a_{1}, a_{2}\right)=\left\{\left\{a_{1}\right\},\left\{a_{1}, a_{2}\right\}\right\} \in X^{n+2}=X^{n+2 \cdot 2-2} .
$$

Reciprocamente, se $\left(a_{1}, a_{2}\right) \in X^{n+2}$, então, pela letra $(g)$, os conjuntos $\left\{a_{1}\right\}$ e $\left\{a_{1}, a_{2}\right\}$ pertencerão a $X_{n+1}$, e, como $X$ é um conjunto base, tais conjuntos serão 
$X_{\omega}$-conjuntos de nível $n+1$, resultando em $a_{1}, a_{2} \in X_{n}$ e provando que a equivalência desejada é válida no caso $m=2$. Suponha que ela é válida para um número natural $m \geqslant 2$ qualquer, e considere o caso em que a sequência dos $a_{i}$ tem $m+1$ elementos. Se $a_{1}, a_{2} \in X_{n}$ e $a_{i} \in X_{n+2 i-4}(\forall i \geqslant 3)$, então teremos $a_{m+1} \in X_{n+2 m-2}$ e $\left(a_{1} \ldots a_{m}\right) \in X^{n+2 m-2}$ pela hipótese de indução, implicando em

$$
\left(a_{1} \ldots a_{m} a_{m+1}\right)=\left(\left(a_{1} \ldots a_{m}\right), a_{m+1}\right) \in X^{n+2 m-2+2}=X^{n+2(m+1)-2}
$$

como vimos no caso $m=2$. A prova da implicação recíproca é análoga, finalizando a indução.

(i) Temos $R\langle A\rangle \subset B \subset X_{n-1}$. Pela letra $(f)$, os produtos cartesianos $A \times B$ e $B \times A$ estão contidos em $X_{n+1}$, e, consequentemente, as relações $R$ e $R^{-1}$ também estão.

(j) Todo elemento de $R$ é um $X_{\omega}$-conjunto, e se $(x, y) \in R \subset X^{n-1}$, então $x, y \in X_{n-3}$ pela letra $(h)$. Assim, os conjuntos $A$ e $R\langle A\rangle$ estão contidos em $X_{n-3}$, a relação inversa $R^{-1}$ é uma relação binária em $X_{n-3}$, e, como $X_{n-3} \in X^{n-2}$, pela letra $(f)$ temos $R^{-1} \subset X_{n-1}$.

(k) Como $X_{n} \in X^{n+1} \mathrm{e}$

$$
R \circ H \subset X_{n} \times X_{n} \in X^{n+3},
$$

pela letra $(f)$ temos $R \circ H \in X^{n+3}$.

(l) Pela letra $(j)$, os conjuntos $I$ e $\left\{A_{i}\right\}_{i \in I}$ têm nível $n-2$ em $X^{\omega}$, e, pela letra $(d)$, temos $\bigcup_{i \in I} A_{i} \in X^{n-3}$. Como

$$
\prod_{i \in I} A_{i} \subset \mathcal{P}\left(I \times \bigcup_{i \in I} A_{i}\right)
$$

o resultado segue pelas letras $(a)$ e $(f)$.

Note que $X_{\omega}$ satisfaz às condições 1,2 e 3 da definição indutiva apresentada na Seção 2.2, mas a condição 4 não é observada, ou seja, existem uniões de $X_{\omega}$-conjuntos que não são $X_{\omega}$-conjuntos. Por exemplo, o conjunto $X_{\omega}$ é a união dos níveis $X_{0} X_{1} X_{2} \ldots$, e temos $X_{\omega} \notin X^{\omega}$ (Teorema B.5). Para que uma união de $X_{\omega}$-conjuntos seja um $X_{\omega}$-conjunto, a coleção das classes envolvidas nessa união deve formar um $X_{\omega}$-conjunto (Teorema 4.8, Letra $(d)$ ).

Pela letra $(h)$ do Teorema 4.8 , nota-se que se $a_{1} \ldots a_{m} \in X_{\omega}(\operatorname{com} m \geqslant 2)$ e se a $m$-tupla $\left(a_{1} \ldots a_{m}\right)$ pertencer a $X^{k}$, então cada $a_{i}$ pertencerá ao $(k-2)$-ésimo nível em $X_{\omega}$. 
Podemos hierarquizar os $X_{\omega}$-objetos de acordo com o menor nível que cada elemento aparece na sequência dos $X_{n}$. Temos, assim, a seguinte definição:

Definição 4.9. Seja $x \in X_{\omega}$. O posto de $x$ em $X_{\omega}$, denotado por posto ${ }_{X_{\omega}}(x)$, é o menor número inteiro $n \geqslant-1$ tal que $x \in X_{n+1}$.

Apenas os átomos têm posto -1 , e, para cada número inteiro não negativo $n$, o conjunto dos $X_{\omega}$-objetos de posto $n$ é dado pela diferença

$$
X_{n+1}-X_{n}=X^{n+1}-X^{n}
$$

a qual tem posto $n+1$ em $X_{\omega}$. Como assumimos que $X$ é um conjunto base, temos posto $(\emptyset)=\operatorname{posto}(X)=0$.

Proposição 4.10. Se A for um $X_{\omega}$-conjunto não nulo, então

$$
\operatorname{posto}_{X_{\omega}}(A)=\sup \left\{\operatorname{posto}_{X_{\omega}}(a)+1 \vdots a \in A\right\} .
$$

Demonstração. Seja $n$ o posto de $A$ em $X_{\omega}$, e seja $N:=\left\{\operatorname{posto}_{X_{\omega}}(a)+1 \vdots a \in A\right\}$. Assim, temos $A \in X^{n+1}$ e $A \subset X_{n}$, resultando que se $a$ for um elemento de $A$, então

$$
\operatorname{posto}_{X_{\omega}}(a)+1 \leqslant(n-1)+1=n=\operatorname{posto}_{X_{\omega}}(A)
$$

ou seja, o número $n$ é uma cota superior de $N$ em $\mathbb{Z}$. Suponha que $m$ é uma cota superior de $N$ com $m<n$. Se $a \in A$, então posto $_{X_{\omega}}(a)+1 \leqslant m$ e $a \in X_{m}$, resultando em $A \subset X_{m}$, $A \in X^{m+1}$ e $n+1 \leqslant m+1$, o que é absurdo, provando que $n$ é a menor cota superior de $N$ em $\mathbb{Z}$.

A função dada por $x \mapsto \operatorname{posto}_{X_{\omega}}(x)$ com domínio $X_{\omega}$ é crescente com respeito à relação de pertinência.

Corolário 4.11. Se $a, b \in X_{\omega}$ com $a \in b$, então

$$
\operatorname{posto}_{X_{\omega}}(a)<\operatorname{posto}_{X_{\omega}}(b)
$$

Exemplo 4.12. Se $Y:=\{\emptyset,\{\emptyset\}\}$, então $Y$ não é um conjunto base. Nesse caso, temos

$$
\operatorname{posto}_{Y_{\omega}}(\{\emptyset\})=-1 \neq 0=\sup \left\{\operatorname{posto}_{Y_{\omega}}(a)+1 \vdots a \in\{\emptyset\}\right\}
$$

e

$$
\operatorname{posto}_{Y_{\omega}}(\emptyset)=-1=\operatorname{posto}_{Y_{\omega}}(\{\emptyset\}),
$$

mostrando que a Proposição 4.10 e o Corolário 4.11 não seriam necessariamente válidos caso $X$ não fosse um conjunto base. 


\subsection{Monomorfismos Não Standard}

O conceito de $X_{\omega}$-objeto (Definição 4.1) e a ideia de satisfatibilidade de uma $L_{\epsilon}$-estrutura sobre uma $L_{\epsilon}$-fórmula com parâmetros (Definição A.34) viabilizam a elaboração de uma caracterização formal e generalizada da noção de monomorfismo não standard apresentada na Seção 2.4. Para isso, as condições relacionadas a $X$ (Seção 2.3) são substituídas por $L_{\epsilon}$-fórmulas com quantificadores limitados (Definições A.14 e A.16), e a noção de verdade ou falsidade de uma condição é manifestada por condições da forma $M \models \phi$, onde as $L_{\epsilon}$-estruturas $M$ utilizadas são definidas a seguir.

Definição 4.13. A $L_{\epsilon}$-estrutura $\left(X_{\omega}, \mathrm{E}_{X_{\omega}}\right)$ é chamada de superestrutura com átomos em $X$ e é denotada por $V(X)$.

Como todo conjunto finito de elementos de $X_{\omega}$ está contido algum nível $X_{n}$, podemos aplicar livremente várias notações que reduzem a aparência das fórmulas consideradas, tais como as Notações A.29, A.30 e A.31. Isso nos permitirá representar em $L_{\epsilon}$-fórmulas com quantificadores limitados os conjuntos finitos, as $n$-tuplas ordenadas, as imagens de funções sobre elementos dos seus domínios, os tipos de funções e diversas outras noções e operações conjuntistas.

Observação 4.14. Por efeito de $X$ ser um conjunto base, é impossível manifestar com alguma relevância as propriedades internas dos $X_{\omega}$-átomos por meio de condições da forma $V(X) \models \phi$, embora, tecnicamente, eles sejam conjuntos em NBG. A título de exemplo, se $\phi(x)$ for uma $L_{\epsilon}$-fórmula e se $a$ for um $X_{\omega}$-átomo, então a condição $V(X) \models(\forall x \in a) \phi(x)$ será verdadeira por vacuidade, visto que ela equivale a afirmar

$$
\left(\forall x \in X_{\omega}\right)(x \notin a \vee \phi(x))
$$

e temos $a \cap X_{\omega}=\emptyset$. De fato, as condições da forma $x \in a$ para $a \in X$ sempre terão valor lógico de falsidade sob a interpretação da $L_{\epsilon}$-estrutura $V(X)$, ou seja, os átomos em uma superestrutura comportam-se como se eles não possuíssem estrutura interna.

Definição 4.15. Seja $Y$ um conjunto base. Uma função $*: X_{\omega} \rightarrow Y_{\omega}$ é dita ser um monomorfismo não standard se ela satisfaz às seguintes condições:

(Mon1) $*(X)=Y$;

(Mon2) Para todo subconjunto infinito $A$ de $X$, teremos $*\langle A\rangle \subsetneq *(A)$;

(Mon3) (Princípio da Transferência; PT) Para toda $L_{\epsilon}$-fórmula $\phi\left(x_{1} \ldots x_{n}\right)$ com quantificadores limitados (Definição A.16), temos

$$
V(X) \models \phi\left[a_{1} \ldots a_{n}\right] \Leftrightarrow V(Y) \models \phi\left[*\left(a_{1}\right) \cdots *\left(a_{n}\right)\right]
$$

para quaisquer $a_{1} \ldots a_{n} \in X_{\omega}$. 
O PT implica que a função * é uma imersão do tipo $V(X) \rightarrow V(Y)$ que não é uma imersão elementar (Definição A.42). Em particular, * é injetora. A Notação 2.4 será novamente posta em uso para representar as imagens de $*$ sobre $X_{\omega}$-objetos.

O digrafo SupEstr cuja classe de objetos é dada por

$$
\{V(X) \vdots X \text { é um conjunto base }\}
$$

e cujas setas são os monomorfismos não standard entre superestruturas forma uma categoria grande ${ }^{3}$ tal que todos os morfismos de SupEstr são mono ${ }^{4}$, elucidando a escolha do nome atribuído às funções que satisfazem aos axiomas (Mon1)-(Mon3).

O único exemplo concreto de um monomorfismo não standard que veremos neste trabalho será construído ao longo do Capítulo 5. Tal construção é indubitavelmente a mais simples possível, mas existem diversas outras, incluindo algumas que são caprichosamente projetadas para que a função * obtida observe propriedades extras desejáveis.

Até o final deste capítulo, assumiremos que $*$ é um monomorfismo não standard cujo domínio é a superestrutura $V(X)$. Em virtude do axioma (Mon1), podemos dizer que $*$ é do tipo $V(X) \rightarrow V\left({ }^{*} X\right)$.

Notação 4.16. Se $n \leqslant \omega$ for um ordinal, o $n$-ésimo nível da superestrutura $V\left({ }^{*} X\right)$ será denotado por ${ }^{*} X_{n}$. Tal notação não deve ser confundida com a $*$-transformação do $n$-ésimo nível em $X_{\omega}$, a qual será consistentemente denotada por * $\left(X_{n}\right)$ a fim de evitar ambiguidades notacionais.

Notação 4.17. Ao lidarmos com os axiomas (Mon1)-(Mon3) dos monomorfismos não standard $*: V(X) \rightarrow V\left({ }^{*} X\right)$, apenas duas $L_{\epsilon}$-estruturas serão de interesse, e.g. $V(X)$ e $V\left({ }^{*} X\right)$. Por tal motivo, convém simplificar a notação para as condições das formas $V(X) \models \phi$ e $V\left({ }^{*} X\right) \models \phi$, as quais serão respectivamente denotadas por $\models \phi \mathrm{e}^{*} \models \phi$ neste capítulo.

Se $x$ for um $X_{\omega}$-átomo (resp. $X_{\omega}$-conjunto), então teremos $\models x \in X$ (resp. $\models x \notin X)$, resultando em ${ }^{*} \models{ }^{*} x \in{ }^{*} X$ (resp. $\left.{ }^{*} \models{ }^{*} x \notin{ }^{*} X\right)$, ou seja, ${ }^{*} x$ será um ${ }^{*} X_{\omega}$-átomo (resp. ${ }^{*} X_{\omega}$-conjunto).

Como * é uma imersão entre $L_{\epsilon}$-estruturas, seria razoável identificar cada $X_{\omega}$-objeto com a sua $*$-transformação. Porém, a identificação de um $X_{\omega}$-conjunto $A$ com o ${ }^{*} X_{\omega}$-conjunto ${ }^{*} A$ pode gerar muitas complicações e despropósitos, uma vez que, em geral, os conjuntos $A \mathrm{e}^{*} A$ apresentam traços estruturais consideravelmente discrepantes, tornando, assim, inquietante a ideia de denotá-los pelos mesmos símbolos. Seria incabível denotarmos o conjunto dos números hiper-reais pelo símbolo $\mathbb{R}$, por exemplo. Além

3 Uma categoria é dita ser grande se a classe dos seus objetos for uma classe própria.

4 Um morfismo $f: A \rightarrow B$ em uma categoria $C$ é dito ser mono se, para quaisquer morfismos $u, v: C \rightarrow A$ em $C$, a condição $f \circ u=f \circ v$ implica $u=v$. 
disso, o ${ }^{*} X_{\omega}$-conjunto $*\langle A\rangle$ é uma cópia ${ }^{5}$ de $A$, tornando-o um candidato mais pertinente em * $X^{\omega}$ para ser identificado com $A$. Não há inconveniência alguma, no entanto, em identificar cada $X_{\omega}$-átomo a com a sua $*$-transformação ${ }^{*} a$, visto que os átomos são desprovidos de estrutura interna sob a interpretação da superestrutura na qual estão inseridos (Observação 4.14). Assim, adotaremos a seguinte notação:

Notação 4.18. Cada $X_{\omega}$-átomo será identificado com a sua respectiva *-transformação, e escreveremos

$$
a={ }^{*} a(\forall a \in X) .
$$

Decorre dessa notação a identificação de cada subconjunto $S$ de $X$ com a imagem $*\langle S\rangle$, a qual está contida em ${ }^{*} X$.

Notação 4.19. Quando considerarmos que o conjunto $X$ é infinito, adotaremos uma injeção ${ }^{6}$ fixa do tipo $\mathbb{Z} \rightarrow X$ e identificaremos a imagem dessa função com o conjunto dos números inteiros. Assim, escreveremos $\mathbb{Z} \subset X$, e, em virtude da Notação 4.18, também escreveremos $\mathbb{Z} \subset{ }^{*} X$. Como os produtos cartesianos da forma $\mathbb{Z}^{n}$ são $X_{\omega}$-conjuntos e ${ }^{*} X_{\omega}$-conjuntos (Teorema 4.8, Letra $(f)$ ), temos que qualquer relação $n$-ária em $\mathbb{Z}$ é um $X_{\omega}$-conjunto e um ${ }^{*} X_{\omega}$-conjunto.

No Capítulo 2, tratamos informalmente do caso $X=\mathbb{R}$, ou seja, investigamos os monomorfismos não standard do tipo $*: \mathbb{R}_{\omega} \rightarrow^{*} \mathbb{R}_{\omega}$. A tabela a seguir resume a correlação existente entre os conceitos desenvolvidos neste capítulo e no Teorema 2.3.

Tabela 4 - Correspondências entre os postulados do Teorema 2.3 e os axiomas, convenções e teoremas do Capítulo 4.

\begin{tabular}{|c|c|c|}
\hline Postulado & Axioma/convenção & Observação \\
\hline Letra $(a)$ & $\operatorname{dom}(*)=\mathbb{R}_{\omega}$ & Os objetos relacionados a $\mathbb{R}\left(\right.$ resp. $\left.{ }^{*} \mathbb{R}\right)$ foram \\
\hline Letra $(b)$ & $\operatorname{Im}(*) \subset * \mathbb{R}_{\omega}$ & substituídos pelos $\mathbb{R}_{\omega}$-objetos (resp. ${ }^{*} \mathbb{R}_{\omega}$-objetos). \\
\hline Letra $(c)$ & Notação 4.18 & $\begin{array}{l}\text { A igualdade }{ }^{*} x=x \text { para } x \in \mathbb{R} \text { passou } \\
\text { a ser uma identificação notacional. }\end{array}$ \\
\hline Letra $(d)$ & (Mon1) & - \\
\hline Letra $(e)$ & Corolário 4.28 & -1 \\
\hline Letra $(f)$ & (Mon3) & $\begin{array}{c}\text { As condições completamente abertas relacionadas } \\
\text { a } \mathbb{R} \text { foram substituídas pelas } L_{\epsilon} \text {-fórmulas, as quais } \\
\text { são avaliadas via relação de satisfatibilidade } \\
\text { sobre superestruturas. }\end{array}$ \\
\hline
\end{tabular}

Nas seções a seguir, averiguaremos que os demais postulados considerados no Capítulo 2, viz. a letra (e) do Teorema 2.3 e os Teoremas 2.9, 2.26, 2.27 e 2.31, são consequências de outras definições e dos axiomas (Mon1)-(Mon3).

5 A restrição $* \uparrow_{A}: A \rightarrow *\langle A\rangle$ é uma bijeção.

6 A imersão de $\mathbb{Z}$ em $X$ é suficiente para os propósitos deste capítulo, mas poderíamos ter fixado uma injeção $\mathbb{Q} \rightarrow X$, visto que os conjuntos $\mathbb{Z}$ e $\mathbb{Q}$ são equipotentes. 


\subsection{Objetos Internos e Externos}

Duas categorizações para os ${ }^{*} X_{\omega}$-objetos são indispensáveis no estudo dos monomorfismos não standard.

\section{Definição 4.20.}

1. $\mathrm{Um}^{*} X_{\omega}$-objeto é dito ser standard se ele pertence à imagem $\operatorname{Im}(*)$ do monomorfismo não standard *. Caso contrário, dizemos que ele é não standard;

2. $\mathrm{Um}^{*} X_{\omega}$-objeto é dito ser interno se ele pertence a algum ${ }^{*} X_{\omega}$-objeto standard. Caso contrário dizemos que ele é externo.

Todo objeto standard é interno, e, em particular, todo ${ }^{*} X_{\omega}$-átomo é interno. Como as $*$-transformações dos $X_{\omega}$-átomos são ${ }^{*} X_{\omega}$-átomos, e como ${ }^{*} X$ é um conjunto base, temos que um ${ }^{*} X_{\omega}$-objeto $a$ é interno se, e somente se, existe um $X_{\omega}$-conjunto $A$ tal que $a \in{ }^{*} A$. Veremos que se $X$ for finito, então todo ${ }^{*} X_{\omega}$-objeto será standard (Exemplo 4.33). Diversos exemplos de objetos standard, não standard, internos e externos foram mostrados no Capítulo 2.

Proposição 4.21. O conjunto dos ${ }^{*} X_{\omega}$-objetos internos é dado pela união $\bigcup_{n<\omega}^{*}\left(X_{n}\right)$.

Demonstração. Como $X_{n} \in X^{\omega}(\forall n<\omega)$, todo elemento da união do enunciado é interno. Suponha que $a$ é um ${ }^{*} X_{\omega}$-objeto interno. Assim, existe um $B \in X^{m} \operatorname{com} m<\omega$ tal que $a \in{ }^{*} B$. Como $\models(\forall x \in B) x \in X_{m-1}$, pelo PT temos $* \models\left(\forall x \in{ }^{*} B\right) x \in{ }^{*}\left(X_{m-1}\right)$, implicando em

$$
a \in^{*}\left(X_{m-1}\right) \subset \bigcup_{n<\omega}^{*}\left(X_{n}\right)
$$

Notação 4.22 .

- Semelhantemente à maneira como fizemos no Capítulo 2, denotaremos por $\mathbb{I}_{\omega} \mathrm{o}$ conjunto $\bigcup_{n<\omega}^{*}\left(X_{n}\right)$ dos ${ }^{*} X_{\omega}$-objetos internos;

- Cada imagem * $\left(X_{n}\right)$ com $n<\omega$ é dita ser o $n$-ésimo nível de $\mathbb{I}_{\omega}$ e é denotada por $\mathbb{I}_{n}$. Dizemos que cada elemento de $\mathbb{I}_{n}$ tem nível $n$ em $\mathbb{I}_{\omega}$;

- Cada diferença $\mathbb{I}_{n}-{ }^{*} X$ com $n \leqslant \omega$ é denotada por $\mathbb{I}^{n}$. Dizemos que cada elemento de $\mathbb{I}^{n}$ tem nível $n$ em $\mathbb{I}^{\omega}$.

Claramente, temos $\mathbb{I}_{0}={ }^{*} X, \mathbb{I}^{0}=\emptyset$, e, como

$$
\models\left(\forall x \in X_{n}\right)\left(x \notin X \longleftrightarrow x \in X^{n}\right),
$$


temos $\mathbb{I}^{n}={ }^{*}\left(X^{n}\right)$. Para um $n<\omega$, aplicando o PT às condições verdadeiras $X_{n}, X^{n} \in X^{n+1}$ e $X^{n} \subset X_{n} \subset X_{n+1}$ obtém-se $\mathbb{I}_{n}, \mathbb{I}^{n} \in \mathbb{I}^{n+1}$ e $\mathbb{I}^{n} \subset \mathbb{I}_{n} \subset \mathbb{I}_{n+1}$.

Haja vista que

$$
\models\left(\forall A \in X^{n}\right)(\forall x \in A) x \in X_{n-1},
$$

temos que a condição $A \in \mathbb{I}^{n}$ implica $A \subset \mathbb{I}_{n-1}$, e se todo elemento de $A$ for $\mathrm{um}^{*} X_{\omega^{-}}$conjunto, então a a condição $A \in \mathbb{I}^{n}$ implicará $A \subset \mathbb{I}^{n-1}$. Em particular, todo elemento de um conjunto interno é interno, solidificando a validez do Teorema 2.26. Existem subconjuntos de $\mathbb{I}_{n-1}$ que não pertencem a $\mathbb{I}^{n}$, e.g. no caso $X=\mathbb{R}$ temos que $\mathbb{R}$ é um subconjunto externo de ${ }^{*} \mathbb{R}=\mathbb{I}_{1-1}$ (Corolário 2.35).

Observação 4.23. Analogamente à demonstração da letra $(h)$ do Teorema 4.8, prova-se que se $a_{1} a_{2} \ldots a_{m}$ forem ${ }^{*} X_{\omega}$-objetos tais que a $m$-tupla ordenada $\left(a_{1} a_{2} \ldots a_{m}\right)$ pertence ao nível $\mathbb{I}^{n}$ com $n<\omega$, então cada $a_{i}$ pertencerá ao nível $\mathbb{I}_{n-2}$.

Com tais decorrências da Definição 4.20, temos as ferramentas necessárias para demonstrar a versão formal do Teorema 2.27.

Teorema 4.24 (Princípio da Definição Interna; PDI). Seja

$$
\phi\left(x_{1} \ldots x_{n}, y_{1} \ldots y_{p}\right)
$$

uma $L_{\epsilon}$-fórmula com quantificadores limitados, seja $R$ uma relação $n$-ária em ${ }^{*} X_{\omega}$ com $R \in \mathbb{I}^{m}(m<\omega)$, e sejam $a_{1} \ldots a_{p} \in \mathbb{I}_{k}(k<\omega)$. O conjunto

$$
S:=\left\{\left(t_{1} \ldots t_{n}\right) \in R: * \models \phi\left(t_{1} \ldots t_{n}, a_{1} \ldots a_{p}\right)\right\}
$$

tem nível $m$ em $\mathbb{I}^{\omega}$.

Demonstração. Se $H$ for uma relação $n$-ária em $X_{\omega} \operatorname{com} H \in X^{m}$, e se $\left(t_{1} \ldots t_{n}\right) \in H$, então, como cada elemento de $H$ é um $X_{\omega}$-conjunto, teremos $H \subset X^{m-1}$, $\left(t_{1} \ldots t_{n}\right) \in X^{m-1}$, cada coordenada $t_{i}$ pertencerá a $X_{m-3}$ (Teorema 4.8 , Letra $(h)$ ), e para quaisquer $c_{1} \ldots c_{p} \in X_{k}$ teremos

$$
\left\{\left(t_{1} \ldots t_{n}\right) \in H \vdots \models \phi\left(t_{1} \ldots t_{n}, c_{1} \ldots c_{p}\right)\right\} \in X^{m}
$$

Assim, a condição

$$
\models\left(\forall H \in X^{m}\right)\left(\forall y_{1} \ldots y_{p} \in X_{k}\right)\left(\exists I \in X^{m}\right) \psi\left(I, X_{m-1}, H, y_{1} \ldots y_{p}, X_{m-3}\right)
$$

é verdadeira, onde a $L_{\epsilon}$-fórmula $\psi\left(I, U, H, y_{1} \ldots y_{p}, V\right)$ é lida informalmente como

$$
" I=\left\{\left(t_{1} \ldots t_{n}\right) \in H \vdots \models \phi\left(t_{1} \ldots t_{n}, y_{1} \ldots y_{p}\right)\right\} "
$$


e é dada formalmente por

$$
(\forall u \in U)\left[u \in I \longleftrightarrow\left(u \in H \wedge\left(\exists x_{1} \ldots x_{n} \in V\right)\left(u=\left(x_{1} \ldots x_{n}\right) \wedge \phi\left(x_{1} \ldots x_{n}, y_{1} \ldots y_{p}\right)\right)\right)\right] .
$$

Pelo PT, existe um $I \in \mathbb{I}^{m}$ tal que $* \models \psi\left(I, \mathbb{I}_{m-1}, R, a_{1} \ldots a_{p}, \mathbb{I}_{m-3}\right)$. Claramente temos $I \subset S$, e se $\left(t_{1} \ldots t_{n}\right)$ for uma $n$-tupla ordenada pertencente a $S$, então, como cada

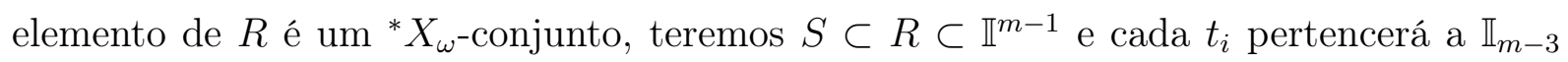
(Observação 4.23), resultando em $\left(t_{1} \ldots t_{n}\right) \in I$ e provando que $S=I \in \mathbb{I}^{m}$.

Ao aplicarmos o PDI no caso $n=1$ e $m \geqslant 1$, basta assumirmos que $R$ é o nível $\mathbb{I}_{m-1}$ em $\mathbb{I}_{\omega}$.

Teorema 4.25 (Propriedades Conjuntistas dos Objetos Internos).

(a) Se $a_{1} a_{2} \ldots a_{m} \in \mathbb{I}_{n}$, então $\left\{a_{1} a_{2} \ldots a_{m}\right\} \in \mathbb{I}^{n+1}$ e $\left(a_{1} a_{2} \ldots a_{m}\right) \in \mathbb{I}^{n+2 m-2}$;

(b) $\quad$ Se $\mathcal{A} \subset \mathbb{I}^{\omega}$ e $\mathcal{A} \in \mathbb{I}^{n}$, então $\bigcup \mathcal{A}, \bigcap \mathcal{A} \in \mathbb{I}^{n-1}$;

(c) Se $A, B \in \mathbb{I}^{n}$, então $A \cup B, A \cap B, A-B \in \mathbb{I}^{n}$;

(d) Se $A, B \in \mathbb{I}^{n}$, então $A \times B \in \mathbb{I}^{n+2}$;

(e) Se $R$ for uma relação binária em ${ }^{*} X_{\omega}$ pertencente $a \mathbb{I}^{n}$, e se $P$ for um subconjunto interno de $\operatorname{dom}(R)$, então $R$ será uma relação binária em $\mathbb{I}_{n-3}$, os conjuntos $\operatorname{dom}(R)$ e $R\langle P\rangle$ e terão nível $n-2$ em $\mathbb{I}^{\omega}$, a relação inversa $R^{-1}$ terá nível $n$ em $\mathbb{I}^{\omega}$, e teremos

$$
\mathcal{Q}:=\{R\langle\{x\}\rangle: x \in P\} \in \mathbb{I}^{n+1} .
$$

Em particular, se $f$ for uma função que é uma relação binária em ${ }^{*} X_{\omega}$ pertencente $a \mathbb{I}^{n}$, e se $\alpha \in \operatorname{dom}(f)$, então $f(\alpha) \in \mathbb{I}_{n-3} ;$

(f) Se $R$ e $H$ forem duas relações binárias $\mathrm{em}^{*} X_{\omega}$ pertencentes a $\mathbb{I}^{n}$, então a composição $R \circ H$ terá nível $n$ em $\mathbb{I}^{\omega}$;

(g) Se $\left\{A_{i}\right\}_{i \in I}$ for uma família de ${ }^{*} X_{\omega}$-conjuntos pertencente a $\mathbb{I}^{n}$ com $I \in{ }^{*} X^{\omega}$ finito, então o produto cartesiano $\prod_{i \in I} A_{i}$ terá nível $n+2$ em $\mathbb{I}^{\omega}$.

Demonstração. Sejam $\phi_{1} \phi_{2} \ldots \phi_{13}$ as seguintes $L_{\epsilon}$-fórmulas:

- $\phi_{1}\left(x, y_{1} y_{2} \ldots y_{m}\right):=x=y_{1} \vee x=y_{2} \vee \cdots \vee x=y_{m}$;

- $\phi_{2}(x, U):=(\exists y \in U) x \in y$;

- $\phi_{4}(x, U, V):=x \in U \vee x \in V$;

- $\phi_{3}(x, U):=(\forall y \in U) x \in y$;

- $\phi_{5}(x, U, V):=x \in U \wedge x \in V$; 
- $\phi_{6}(x, U, V):=x \in U \wedge x \notin V$;

- $\phi_{9}(x, U, N):=(\exists x \in N)(x, y) \in R$;

- $\phi_{7}\left(x_{1}, x_{2}, U, V\right):=x_{1} \in U \wedge x_{2} \in V$;

- $\phi_{8}(x, U, T):=(\exists y \in T)(x, y) \in U ; \quad \bullet \phi_{10}\left(x_{1}, x_{2}, U\right):=\left(x_{2}, x_{1}\right) \in U$;

- $\phi_{11}(x, U, V):=(\forall y \in x) y \in V \wedge(\exists u \in U)(\forall y \in V)(y \in x \longleftrightarrow(u, y) \in x)$;

- $\phi_{12}\left(x_{1}, x_{2}, U, V, T\right):=(\exists z \in T)\left(\left(x_{1}, z\right) \in V \wedge\left(z, x_{2}\right) \in U\right)$;

- $\phi_{13}(x, J, U, F):=x: J \rightarrow U \wedge(\forall j \in J) x(j) \in F(j)$,

onde aplicamos várias abreviações análogas às mencionadas nas Notações A.29, A.30 e A.31 para representar algumas dessas fórmulas.

(a) Como $\left\{a_{1} a_{2} \ldots a_{m}\right\} \subset \mathbb{I}_{n} \in \mathbb{I}^{n+1}$, o resultado $\left\{a_{1} a_{2} \ldots a_{m}\right\} \in \mathbb{I}^{n+1}$ segue ao aplicarmos o PDI à fórmula $\phi_{1}$, e a condição $\left(a_{1} a_{2} \ldots a_{m}\right) \in \mathbb{I}^{n+2 m-2}$ é provada diretamente por indução sobre $m$.

(b) Se $A \in \mathcal{A} \subset \mathbb{I}^{n-1}$, então $A \subset \mathbb{I}_{n-2}$, implicando em $\bigcup \mathcal{A}, \cap \mathcal{A} \subset \mathbb{I}_{n-2} \in \mathbb{I}^{n-1}$, e o resultado segue ao aplicarmos o PDI às fórmulas $\phi_{2}$ e $\phi_{3}$.

(c) Como $A, B \subset \mathbb{I}_{n-1}$, temos $A \cup B, A \cap B, A-B \subset \mathbb{I}_{n-1} \in \mathbb{I}^{n}$, e o resultado segue ao aplicarmos o PDI às fórmulas $\phi_{4}, \phi_{5}$ e $\phi_{6}$.

(d) Temos $A, B \subset \mathbb{I}_{n-1}$. Se $(x, y) \in A \times B$, então $x, y \in \mathbb{I}_{n-1}$ e $(x, y) \in \mathbb{I}^{n+1}$ pela letra (a), implicando em $A \times B \subset \mathbb{I}^{n+1} \in \mathbb{I}^{n+2}$. Assim, o resultado segue ao aplicarmos o $\mathrm{PDI}$ à fórmula $\phi_{7}$.

(e) Se $(x, y) \in R$, então $(x, y) \in \mathbb{I}^{n-1}$, os conjuntos $\{x\}$ e $\{x, y\}$ terão nível $n-2$ em $\mathbb{I}^{\omega}$, e teremos $x, y \in \mathbb{I}_{n-3}$, resultando em $\operatorname{dom}(R), R\langle P\rangle \subset \mathbb{I}_{n-3} \in \mathbb{I}^{n-2}$. Assim, aplicando o PDI às fórmulas $\phi_{8}$ e $\phi_{9}$, temos $\operatorname{dom}(R), R\langle P\rangle \in \mathbb{I}^{n-2}$, onde a variável $T$ deve ser substituída por $\mathbb{I}_{n-3}$ em $\phi_{8}$ e a variável $N$ deve ser substituída por $P$ em $\phi_{9}$. Nesse caso, como $(y, x) \in \mathbb{I}^{n-1}$ pela letra $(a)$, temos $R^{-1} \subset \mathbb{I}^{n-1} \in \mathbb{I}^{n}$, e a condição $R^{-1} \in \mathbb{I}^{n}$ segue ao aplicarmos o PDI à fórmula $\phi_{10}$.

Se $x \in P$, então $x$ e $\{x\}$ serão internos, resultando que $R\langle\{x\}\rangle$ terá nível $n-2$ em $\mathbb{I}^{\omega}$. Assim, temos $\mathcal{Q} \subset \mathbb{I}^{n-2} \in \mathbb{I}^{n-1}$, e o resultado $\mathcal{Q} \in \mathbb{I}^{n-1}$ segue ao aplicarmos o PDI à fórmula $\phi_{11}$, onde as variáveis $U$ e $V$ devem ser substituídas por $P$ e $\mathbb{I}_{n-3}$, respectivamente. 
(f) Pela letra $(e)$, temos que $R$ e $H$ são relações binárias em $\mathbb{I}_{n-3}$, resultando em $R \circ H \subset \mathbb{I}_{n-3} \times \mathbb{I}_{n-3} \in \mathbb{I}^{n}$ pela letra $(d)$. O resultado desejado segue ao aplicarmos o PDI à fórmula $\phi_{12}$, onde a variável $T$ deve ser substituída por $\mathbb{I}_{n-3}$.

(g) Seja $f \in \prod_{i \in I} A_{i}$ e seja $(i, x) \in f$. Pela letra $(e)$, temos $I \subset \mathbb{I}_{n-3}$ e $A_{i} \in \mathbb{I}^{n-3}(\forall i \in I)$, resultando em $A_{i} \subset \mathbb{I}_{n-2}(\forall i \in I)$ e $f \subset \mathbb{I}_{n-3} \times \mathbb{I}_{n-2} \subset \mathbb{I}^{n}$. Como $I$ é finito, temos que $f$ é um conjunto finito de elementos de $\mathbb{I}_{n}$, implicando em $f \in \mathbb{I}^{n+1}$ pela letra (a), e provando que $\prod_{i \in I} A_{i} \subset \mathbb{I}^{n+1} \in \mathbb{I}^{n+2}$. O resultado segue ao aplicarmos o PDI à fórmula $\phi_{13}$, onde a variável $U$ deve ser substituída por $\mathbb{I}_{n-2}$.

Pela letra $(a)$, temos que $\mathrm{um}^{*} X_{\omega}$-conjunto finito será interno se, e somente se, cada um dos seus elementos for interno.

Exemplo 4.26. Se $A$ for um $^{*} X_{\omega}$-conjunto interno e se $b$ for um $^{*} X_{\omega}$-objeto interno, então a função constante do tipo $f: A \rightarrow\{b\}$ será interna, visto que $f=A \times\{b\}$ (Teorema 4.25 , Letras $(a)$ e $(d))$.

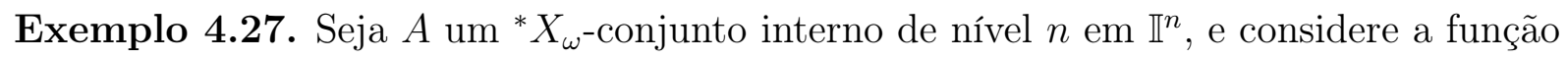
identidade $\operatorname{id}_{A}: A \rightarrow A$. Como o produto cartesiano $A \times A$ é interno (Teorema 4.25, Letra $(d))$ e como $\operatorname{id}_{A} \subset A \times A$, pelo PDI temos que id ${ }_{A}$ é interna, visto que tal função é dada por

$$
\operatorname{id}_{A}=\left\{\left(t_{1}, t_{2}\right) \in A \times A \vdots * \models t_{1}=t_{2}\right\}
$$

Se existir um $X_{\omega^{-}}$conjunto infinito, então $X$ será infinito (Exemplo 4.2), existirá uma cópia de $\mathbb{N}$ contida em $X$ e ${ }^{*} X$ (Notação 4.19), e, pelo axioma (Mon2), teremos $\mathbb{N} \subsetneq{ }^{*} \mathbb{N}$, implicando que o conjunto $\mathbb{N}$ será um ${ }^{*} X_{\omega}$-conjunto externo (Exemplo 2.32). Esta é a única aplicação direta do axioma (Mon2) neste capítulo.

O corolário a seguir implica na letra (e) do Teorema 2.3, e ele é demonstrado de modo análogo à argumentação discorrida no Corolário 2.35.

Corolário 4.28. Se A for um $X_{\omega}$-conjunto infinito, então $*\langle A\rangle$ será externo. Em particular, teremos $*\langle A\rangle \subsetneq \subsetneq^{*} A$ nesse caso.

Na presença do axioma (Mon3) e quando o conjunto $X$ for infinito, o axioma (Mon2) será equivalente à condição $\mathbb{N} \subsetneq{ }^{*} \mathbb{N}$. 


\subsection{Princípio Geral das $*$-Transformações}

Várias operações matemáticas em $X_{\omega}$ são definidas por $L_{\epsilon}$-fórmulas, de modo que $X_{\omega}$-conjuntos são formados a partir de sequências finitas de parâmetros em $X_{\omega}$. O teorema a seguir oferece uma descrição geral das *-transformações dos resultados dessas operações.

Teorema 4.29 (Princípio Geral das $*$-Transformações ${ }^{7} ;$ PGT). Seja $\phi\left(x, y_{1} \ldots y_{p}\right)$ uma $L_{\epsilon}$-fórmula com quantificadores limitados, e sejam $a_{1} \ldots a_{p} \in X_{\omega}$. Se o conjunto $C$ dado por

$$
C:=\left\{t \in X_{\omega}: \models \phi\left(t, a_{1} \ldots a_{p}\right)\right\}
$$

for um $X_{\omega}$-conjunto, e se $D$ for o conjunto

$$
D:=\left\{t \in{ }^{*} X_{\omega}: * \models \phi\left(t,{ }^{*} a_{1} \ldots{ }^{*} a_{p}\right)\right\}
$$

ent $\tilde{a} O{ }^{*} C=\mathbb{I}_{\omega} \cap D$

Demonstração. Seja $Z$ um $X_{\omega}$-conjunto qualquer. Como

$$
\vDash(\forall x \in Z)\left(x \in C \longleftrightarrow \phi\left(x, a_{1} \ldots a_{p}\right)\right)
$$

pelo PT temos

$$
\models\left(\forall x \in{ }^{*} Z\right)\left(x \in{ }^{*} C \longleftrightarrow \phi\left(x,{ }^{*} a_{1} \ldots{ }^{*} a_{p}\right)\right)
$$

Em particular, o caso $Z=C$ resulta $\mathrm{em}^{*} C \subset D$. Se $t$ for um elemento interno de $D$, então $t \in{ }^{*} A$ para algum $X_{\omega}$-conjunto $A$, e o caso $Z=A$ implicará $t \in{ }^{*} C$.

Sob as condições do Teorema 4.29 , o conjunto $D$ será um ${ }^{*} X_{\omega}$-conjunto, visto que $C$ e $D$ são definidos pela mesma $L_{\epsilon}$-fórmula e $C$ é um $X_{\omega}$-conjunto. Assim, a mesma argumentação que legitimiza $C$ como um membro de $X^{\omega}$ valerá para justificar que $D$ pertence a ${ }^{*} X^{\omega}$. Se todo elemento de $D$ for um ${ }^{*} X_{\omega}$-conjunto, então ${ }^{*} C=\mathbb{I}^{\omega} \cap D$, e se todo elemento de $D$ for interno, então ${ }^{*} C=D$.

Corolário 4.30 (Princípio da Definição Standard; PDS). Seja

$$
\phi\left(x_{1} \ldots x_{n}, y_{1} \ldots y_{p}\right)
$$

uma $L_{\epsilon}$-fórmula com quantificadores limitados, seja $R$ uma relação $n$-ária em $X_{\omega}$ pertencente a $X^{\omega}$, e sejam $a_{1} \ldots a_{p} \in X_{\omega}$. Sejam

$$
C:=\left\{\left(t_{1} \ldots t_{n}\right) \in R: \models \phi\left(t_{1} \ldots t_{n}, a_{1} \ldots a_{p}\right)\right\}
$$

7 Diferentemente do PDS e do PDI, o enunciado do Teorema 4.29 é raramente explicitamente mencionado na literatura. O autor considera-o tão importante, elucidativo e generalizante quanto aqueles princípios, legitimando, assim, a atribuição de um nome e uma sigla a tal resultado. 


$$
D:=\left\{\left(t_{1} \ldots t_{n}\right) \in{ }^{*} R: * \models \phi\left(t_{1} \ldots t_{n},{ }^{*} a_{1} \ldots{ }^{*} a_{p}\right)\right\}
$$

$\operatorname{Temos}^{*} C=D$.

Demonstração. Seja $k<\omega$ tal que $R \in X^{k}$. Como $C \subset R$, temos que $C$ é um $X_{\omega}$-conjunto, e se $\left(t_{1} \ldots t_{n}\right)$ for uma $n$-tupla de $X_{\omega}$-objetos pertencente a $R$, então $\left(t_{1} \ldots t_{n}\right) \in X^{k-1}$ e $t_{i} \in X_{k-3}(\forall i)$, resultando que o conjunto $C$ pode ser escrito na forma

$$
C=\left\{t \in X_{\omega} \vdots \models t \in R \wedge\left(\exists t_{1} \ldots t_{n} \in X_{k-3}\right)\left(t=\left(t_{1} \ldots t_{n}\right) \wedge \phi\left(t_{1} \ldots t_{n}, a_{1} \ldots a_{p}\right)\right)\right\} .
$$

Seja $E$ o conjunto

$$
E:=\left\{t \in{ }^{*} X_{\omega} \vdots \models t \in{ }^{*} R \wedge\left(\exists t_{1} \ldots t_{n} \in \mathbb{I}_{k-3}\right)\left(t=\left(t_{1} \ldots t_{n}\right) \wedge \phi\left(t_{1} \ldots t_{n},{ }^{*} a_{1} \ldots{ }^{*} a_{p}\right)\right)\right\} .
$$

Todo elemento de $D$ é interno, e, pelo PGT, temos ${ }^{*} C=E$. Como ${ }^{*} R \in \mathbb{I}^{k}$, pela Observação 4.23 nota-se que $E=D$.

Exemplo 4.31. Como $\emptyset=\{t \in X: \models t \neq t\}$, pelo PDS temos

$$
{ }^{*} \emptyset=\left\{t \in{ }^{*} X: * \models t \neq t\right\}=\emptyset \text {. }
$$

Todas as propriedades conjuntistas dos monomorfismos não standard são consequências do PGT, incluindo as que apresentamos como postulados no Teorema 2.31.

Teorema 4.32 (Propriedades Conjuntistas de $*$ ).

1. Sejam $a_{1} a_{2} \ldots a_{n} \in X_{\omega}$ e sejam $A$ e $B$ dois $X_{\omega}$-conjuntos.
(a) ${ }^{*}\left\{a_{1} a_{2} \ldots a_{n}\right\}=\left\{{ }^{*} a_{1}{ }^{*} a_{2} \ldots{ }^{*} a_{n}\right\}$;
(f) ${ }^{*}(A \times B)={ }^{*} A \times{ }^{*} B$;
(b) $\quad *\left(a_{1} a_{2} \ldots a_{n}\right)=\left({ }^{*} a_{1}{ }^{*} a_{2} \ldots{ }^{*} a_{n}\right)$;
(g) $\quad *\left(\mathcal{P}^{n}(A)\right)=\mathbb{I}^{\omega} \cap \mathcal{P}^{n}\left({ }^{*} A\right)$, onde $n$ é um número natural;
(c) ${ }^{*}(A \cup B)={ }^{*} A \cup{ }^{*} B$;
(h) $\quad *\left({ }^{A} B\right)=\mathbb{I}^{\omega} \cap\left({ }^{*} A\right)\left({ }^{*} B\right)$;
(d) ${ }^{*}(A \cap B)={ }^{*} A \cap{ }^{*} B$;
(i) $\quad *(\operatorname{Int}(A, \leqslant) B)=\mathbb{I}^{\omega} \cap \operatorname{Int}\left({ }^{*} A,{ }^{*} \leqslant\right)\left({ }^{*} B\right)$ onde $\leqslant$ é uma relação binária
(e) ${ }^{*}(A-B)={ }^{*} A-{ }^{*} B$; em $A$.

2. Sejam $R$ e $S$ relações binárias em $X_{\omega}$ pertencentes a $X^{\omega}$, e seja $P \subset \operatorname{dom}(R)$.
(j) $S e R \subset A \times B$, então ${ }^{*} R \subset{ }^{*} A \times{ }^{*} B$;
(m) $\quad *(R\langle P\rangle)={ }^{*} R\left\langle{ }^{*} P\right\rangle$;
(k) $\quad *(\operatorname{dom}(R))=\operatorname{dom}\left({ }^{*} R\right)$;
(n) $*\left(R^{-1}\right)=\left({ }^{*} R\right)^{-1}$;
(l) ${ }^{*}(\operatorname{Im}(R))=\operatorname{Im}\left({ }^{*} R\right)$;
(o) ${ }^{*}(R \circ S)={ }^{*} R \circ{ }^{*} S$. 
3. Seja $f: A \rightarrow B$ uma função, e seja $x \in A$.

(p) ${ }^{*} f:{ }^{*} A \rightarrow{ }^{*} B$

(q) $\quad *(f(x))={ }^{*} f\left({ }^{*} x\right)$; (r) ${ }^{*} f$ será injetora se, e somente se, $f$ for injetora;

(s) ${ }^{*} f$ será sobrejetora se, e somente se, $f$ for sobrejetora.

4. Seja $\left\{M_{i}\right\}_{i \in I}$ uma família de $X_{\omega}$-conjuntos pertencente a $X^{\omega}$ com $I \in X^{\omega}$.
(t) $*\left(\bigcup_{i \in I} M_{i}\right)=\bigcup_{i \in \in^{*}} * M_{i}$;
(v) *( $\left.\prod_{i \in I} M_{i}\right)=\mathbb{I}^{\omega} \cap \prod_{i \in{ }^{*} I}^{*} M_{i}$.
(u) $*\left(\bigcap_{i \in I} M_{i}\right)=\bigcap_{i \in{ }^{*} I} * M_{i}$,

5. Sejam $\mathcal{A}$ e $\mathcal{B}$ dois $X_{\omega}$-conjuntos cujos elementos são $X_{\omega}$-conjuntos.
(w) $*\{A \cup B \vdots A \in \mathcal{A}$ e $B \in \mathcal{B}\}=\left\{A \cup B \vdots A \in{ }^{*} \mathcal{A}\right.$ e $\left.B \in{ }^{*} \mathcal{B}\right\}$;
$(\mathbf{x}) *\{A \cap B \vdots A \in \mathcal{A}$ e $B \in \mathcal{B}\}=\left\{A \cap B \vdots A \in^{*} \mathcal{A}\right.$ e $\left.B \in{ }^{*} \mathcal{B}\right\} ;$
(y) $*\{A-B \vdots A \in \mathcal{A}$ e $B \in \mathcal{B}\}=\left\{A-B \vdots A \in{ }^{*} \mathcal{A}\right.$ e $\left.B \in{ }^{*} \mathcal{B}\right\} ;$
(z) $*\{A \times B: A \in \mathcal{A}$ e $B \in \mathcal{B}\}=\left\{A \times B \vdots A \in{ }^{*} \mathcal{A}\right.$ e $\left.B \in{ }^{*} \mathcal{B}\right\}$;
$(\boldsymbol{\alpha}) \quad *(\bigcup \mathcal{A})=\bigcup^{*} \mathcal{A}$
$(\boldsymbol{\beta}) *(\bigcap \mathcal{A})=\bigcap^{*} \mathcal{A}$.

Demonstração. Claramente, temos $(a) \Rightarrow(b)$ e $(f) \Rightarrow(j)$. As condições $(p),(r)$ e $(s)$ são consequências imediatas de $(j)$ e do PT, e as condições $(c)$ e $(d)$ são consequências de $(a),(b),(t)$ e $(u)$. Como

$$
R\langle P\rangle=\operatorname{Im}[R \cap(P \times \operatorname{Im}(R))]
$$

temos que $(m)$ é consequência de $(d),(f)$ e $(l)$.

Sejam $\phi_{1} \phi_{2} \ldots \phi_{19}$ as seguintes $L_{\epsilon}$-fórmulas com quantificadores limitados:

- $\phi_{1}\left(x, y_{1} y_{2} \ldots y_{n}\right):=x=y_{1} \vee x=y_{2} \vee \cdots \vee x=y_{n}$;

- $\phi_{2}(x, U, V):=x \in U \wedge x \notin V$;

- $\phi_{3}(x, U, V):=\left(\exists c_{1} \in U\right)\left(\exists c_{2} \in V\right) x=\left(c_{1}, c_{2}\right)$;

- $\phi_{4}(x, U):=\left(\forall y_{n} \in x\right)\left(\forall y_{n-1} \in y_{n}\right) \cdots\left(\forall y_{1} \in y_{2}\right) y_{1} \in U$; 
- $\phi_{5}(x, U, V):=x: U \rightarrow V$;

- $\phi_{6}(x, U, V, R):=\left(\exists y_{1}, y_{2} \in U\right)\left(x:\left[y_{1}, y_{2}\right]_{R} \rightarrow V\right)$;

- $\phi_{7}(x, U, T):=(\exists y \in T)((x, y) \in U)$;

- $\phi_{8}(x, U, T):=(\exists y \in T)((y, x) \in U)$;

- $\phi_{9}(x, U, T):=\left(\exists c_{1} \in T\right)\left(\exists c_{2} \in T\right)\left(\left(c_{1}, c_{2}\right) \in U \wedge x=\left(c_{2}, c_{1}\right)\right)$;

- $\phi_{10}(x, U, V, T):=\left(\exists c_{1} \in T\right)\left(\exists c_{2} \in T\right)\left(\exists c_{3} \in T\right)\left(\left(c_{1}, c_{2}\right) \in V \wedge\left(c_{2}, c_{3}\right) \in U \wedge x=\left(c_{1}, c_{3}\right)\right)$;

- $\phi_{11}(x, J, F):=(\exists j \in J) x \in F(j)$;

- $\phi_{12}(x, J, F):=(\forall j \in J) x \in F(j)$;

- $\phi_{13}(x, J, F, U):=x: J \rightarrow U \wedge(\forall j \in J) x(j) \in F(j)$;

- $\phi_{14}(x, U, V, T):=\left(\exists Y_{1} \in U\right)\left(\exists Y_{2} \in V\right)(\forall y \in T)\left(y \in x \longleftrightarrow\left(y \in Y_{1} \vee y \in Y_{2}\right)\right)$;

- $\phi_{15}(x, U, V, T):=\left(\exists Y_{1} \in U\right)\left(\exists Y_{2} \in V\right)(\forall y \in T)\left(y \in x \longleftrightarrow\left(y \in Y_{1} \wedge y \in Y_{2}\right)\right)$;

- $\phi_{16}(x, U, V, T):=\left(\exists Y_{1} \in U\right)\left(\exists Y_{2} \in V\right)(\forall y \in T)\left(y \in x \longleftrightarrow\left(y \in Y_{1} \wedge y \notin Y_{2}\right)\right)$;

- $\phi_{17}(x, U, V, T):=\left(\exists Y_{1} \in U\right)\left(\exists Y_{2} \in V\right)(\forall y \in T)\left[y \in x \longleftrightarrow\left(\left(\exists c_{1} \in Y_{1}\right)\left(\exists c_{2} \in Y_{2}\right) y=\left(c_{1}, c_{2}\right)\right)\right]$;

- $\phi_{18}(x, U):=(\exists y \in U) x \in y$;

- $\phi_{19}(x, U):=(\forall y \in U) x \in y$.

Nota-se que

$$
\left\{{ }^{*} a,{ }^{*} b\right\},{ }^{*} A-{ }^{*} B,{ }^{*} A \times{ }^{*} B, \bigcup_{i \in *^{*} I}^{*} M_{i}, \bigcap_{i \in{ }^{*} I}^{*} M_{i}, \bigcup^{*} \mathcal{A}, \bigcap{ }^{*} \mathcal{A} \subset \mathbb{I}_{\omega}
$$

e os resultados das letras $(a),(e),(f),(g),(h),(i),(t),(u),(v),(\alpha)$ e $(\beta)$ seguem ao aplicarmos o PGT às $L_{\epsilon}$-fórmulas $\phi_{1}, \phi_{2}, \phi_{3}, \phi_{4}, \phi_{5}, \phi_{6}, \phi_{11}, \phi_{12}, \phi_{13}, \phi_{18}$ e $\phi_{19}$, respectivamente. Em $\phi_{13}$, a variável $U$ deve ser substituída por um nível em $X_{\omega}$ que contenha a união $\bigcup_{i \in I} M_{i}$.

Como $R, S \in X^{n}$ para algum número natural $n$, temos $R \subset X_{n-3} \times X_{n-3}$ (Teorema 4.8, Letra $(j))$, e as letras $(k),(l),(n)$ e $(o)$ seguem ao aplicarmos o PGT às $L_{\epsilon}$-fórmulas $\phi_{7}, \phi_{8}, \phi_{9}$ e $\phi_{10}$, respectivamente, onde a variável $T$ deve ser substituída pelo nível $X_{n-3}$ em $X_{\omega}$.

Como $\mathcal{A}, \mathcal{B} \subset X^{\omega}$ e $\mathcal{A}, \mathcal{B} \in X^{n}$ para algum número natural $n$, temos $\mathcal{A}, \mathcal{B} \subset X^{n-1}$, e todo elemento de $\mathcal{A}$ ou de $\mathcal{B}$ será um subconjunto de $X_{n-2}$. Portanto, as letras $(w),(x)$ e $(y)$ seguem ao aplicarmos o PGT às $L_{\epsilon}$-fórmulas $\phi_{14}, \phi_{15}$ e $\phi_{16}$, respectivamente, onde a 
variável $T$ deve ser substituída pelo nível $X_{n-2}$ em $X_{\omega}$. Produtos cartesianos de elementos de $X^{n-1}$ são subconjuntos de $X_{n}$ (Teorema 4.8, Letra $(f)$ ), resultando que a letra $(z)$ segue ao aplicarmos o PGT à $L_{\epsilon}$-fórmula $\phi_{17}$, onde a variável $T$ deve ser substituída pelo nível $X_{n}$ em $X_{\omega}$.

Por fim, se $x \in A$, então pelas letras $(a)$ e $(m)$ teremos

$$
\left\{{ }^{*}(f(x))\right\}={ }^{*}\{f(x)\}={ }^{*}(f\langle\{x\}\rangle)={ }^{*} f\left\langle{ }^{*}\{x\}\right\rangle={ }^{*} f\left\langle\left\{{ }^{*} x\right\}\right\rangle=\left\{{ }^{*} f\left({ }^{*} x\right)\right\},
$$

implicando em ${ }^{*}(f(x))={ }^{*} f\left({ }^{*} x\right)$ e provando a letra $(q)$.

As letras $(g),(h)$ e $(i)$ do Teorema 4.32 correspondem ao Teorema 2.31. Assim, finalizamos as demonstrações de todas as afirmações postuladas no Capítulo 2.

Exemplo 4.33. Se $X$ for finito, então todo elemento de ${ }^{*} X={ }^{*} X_{0}$ será standard (Teorema 4.32 , Letra $(a)$ ), e prova-se por indução sobre $n$ que todo elemento do nível ${ }^{*} X_{n}$ será standard. Assim, nesse caso, todo ${ }^{*} X_{\omega}$-objeto será standard, e o monomorfismo não standard $*: V(X) \rightarrow V\left({ }^{*} X\right)$ será um isomorfismo.

\section{Corolário 4.34.}

(a) $\quad \mathbb{I}^{n}=\mathbb{I}^{\omega} \cap^{*} X^{n}$ para cada número natural $n$;

(b) Para cada $x \in X_{\omega}$, temos

$$
\operatorname{posto}_{X_{\omega}}(x)=\operatorname{posto}_{*} X_{\omega}\left({ }^{*} x\right)
$$

Demonstração.

(a) Temos

$$
\mathbb{I}^{n}={ }^{*} \mathcal{P}\left(X_{n-1}\right)=\mathbb{I}^{\omega} \cap \mathcal{P}\left({ }^{*} X_{n-1}\right)=\mathbb{I}^{\omega} \cap{ }^{*} X^{n}
$$

(b) Seja $n$ o posto de $x$ em $X_{\omega}$. Se $n=-1$, então $x \in X \mathrm{e}^{*} x \in{ }^{*} X$, ou seja, ${ }^{*} x$ terá posto $-1 \mathrm{em}^{*} X_{\omega}$. Se $0 \leqslant n$, então

$$
=x \in X^{n+1} \wedge x \notin X^{n}
$$

e, pelo PT, teremos ${ }^{*} x \in \mathbb{I}^{n+1}-\mathbb{I}^{n}$, resultando em $x \in{ }^{*} X^{n+1}-{ }^{*} X^{n}$ pela letra $(a)$, e provando que $\operatorname{posto}_{*} X_{\omega}\left({ }^{*} x\right)=n$.

Temos $* \uparrow_{X}: X \rightarrow{ }^{*} X$ e $* \uparrow_{X^{n}}: X^{n} \rightarrow{ }^{*} X^{n}$ para cada $n<\omega$. A letra $(a)$ do Corolário 4.34 é equivalente à afirmação de que a equação $\mathbb{I}_{n}=\mathbb{I}_{\omega} \cap{ }^{*} X_{n}$ é válida para cada $n<\omega$. Assim, cada nível $\mathbb{I}_{n}$ é o conjunto dos elementos internos no $n$-ésimo nível de ${ }^{*} X_{\omega}$. 


\subsection{Conjuntos Hiperfinitos}

Há uma classe peculiar de ${ }^{*} X_{\omega}$-conjuntos associada à noção de finitude de um conjunto.

Definição 4.35. Um ${ }^{*} X_{\omega}$-conjunto $A$ é dito ser hiperfinito se existe um $X_{\omega^{-}}$conjunto $E$ tal que $A \in{ }^{*} \mathcal{P}_{\omega}(E)$. Se $B$ for um ${ }^{*} X_{\omega}$-conjunto, então o conjunto dos subconjuntos hiperfinitos de $B$ é denotado por $\mathcal{P}_{h f}(B)$.

Todo conjunto hiperfinito é interno.

Exemplo 4.36. O conjunto vazio, $\emptyset$, é hiperfinito, visto que

$$
\emptyset \in\{\emptyset\}=\left\{{ }^{*} \emptyset\right\}={ }^{*}\{\emptyset\}={ }^{*} \mathcal{P}_{\omega}(\emptyset)
$$

Exemplo 4.37. Suponha que o conjunto $X$ é finito. Assim, os $X_{\omega}$-conjuntos e os ${ }^{*} X_{\omega}$-conjuntos são finitos (Exemplo 4.2). Se $A$ for um ${ }^{*} X_{\omega}$-conjunto, então existirá um $X_{\omega}$-conjunto $E$ tal que $A \in{ }^{*} E$ (Exemplo 4.33), e se $n$ for o nível de $E$ em $X^{\omega}$, então teremos $E \subset X_{n-1},{ }^{*} E \subset \mathbb{I}_{n-1}, A \in \mathbb{I}^{n-1}$, e

$$
A \in \mathbb{I}^{\omega} \cap \mathcal{P}\left(\mathbb{I}_{n-2}\right)={ }^{*} \mathcal{P}\left(X_{n-2}\right)={ }^{*} \mathcal{P}_{\omega}\left(X_{n-2}\right),
$$

implicando que $A$ será hiperfinito. Portanto, todo ${ }^{*} X_{\omega}$-conjunto será hiperfinito quando $X$ for finito.

\section{Proposição 4.38.}

(a) ${ }^{*}\left(\mathcal{P}_{\omega}(E)\right)=\mathcal{P}_{h f}\left({ }^{*} E\right)$ para todo $X_{\omega}$-conjunto $E$;

(b) Todo subconjunto interno de um ${ }^{*} X_{\omega}$-conjunto hiperfinito é hiperfinito;

(c) Se $f: A \rightarrow B$ for uma função interna que é uma relação binária em ${ }^{*} X_{\omega}$, e se $S$ for um subconjunto hiperfinito de $A$, então a imagem $f\langle S\rangle$ será hiperfinita.

Demonstração.

(a) Como $\mathcal{P}_{\omega}(E) \subset \mathcal{P}(E)$, temos (Teorema 4.32, Letra $(g)$ )

$$
{ }^{*} \mathcal{P}_{\omega}(E) \subset{ }^{*} \mathcal{P}(E)=\mathbb{I}^{\omega} \cap \mathcal{P}\left({ }^{*} E\right)
$$

implicando em ${ }^{*}\left(\mathcal{P}_{\omega}(E)\right) \subset \mathcal{P}_{h f}\left({ }^{*} E\right)$. Se $A$ for um subconjunto hiperfinito de ${ }^{*} E$,

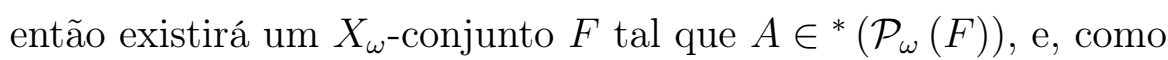

$$
\models\left(\forall A \in \mathcal{P}_{\omega}(F)\right)\left(A \subset E \longrightarrow A \in \mathcal{P}_{\omega}(E)\right),
$$

pelo PT temos $A \in{ }^{*} \mathcal{P}_{\omega}(E)$, provando o resultado desejado. 


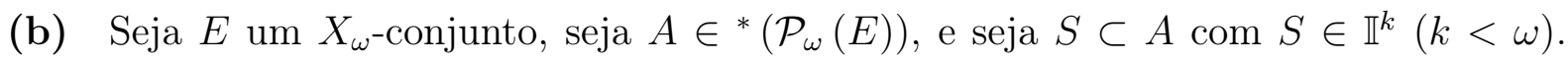
Temos

$$
\models\left(\forall A \in \mathcal{P}_{\omega}(E)\right)\left(\forall S \in X^{k}\right)\left(S \subset A \longrightarrow S \in \mathcal{P}_{\omega}(E)\right)
$$

e o resultado segue pelo $\mathrm{PT}$.

(c) Pelas letras $(c),(d)$ e $(e)$ do Teorema 4.25 , o conjunto $B$ e a restrição

$$
g:=f \uparrow_{S}=f \cap(S \times B)
$$

são internos. Seja $E$ um $X_{\omega}$-conjunto de nível $m<\omega$ tal que $S \in{ }^{*} \mathcal{P}_{\omega}(E)$, e seja $n$ um número natural tal que $B$ e $g$ têm nível $n$ em $\mathbb{I}^{\omega}$. Assim, temos $B \subset \mathbb{I}_{n-1}$ e

$$
\models\left(\forall g \in X^{n}\right)\left(\forall S \in \mathcal{P}_{\omega}(E)\right)\left(g: S \rightarrow X_{n-1} \longrightarrow \operatorname{Im}(g) \in \mathcal{P}_{\omega}\left(X_{n-1}\right)\right),
$$

resultando pelo PT e pela letra $(a)$ em

$$
f\langle S\rangle=\operatorname{Im}(g) \in{ }^{*} \mathcal{P}_{\omega}\left(X_{n-1}\right) .
$$

Até o final deste capítulo, assumiremos que o conjunto $X$ é infinito. A Notação 4.19 será posta em uso.

Exemplo 4.39. Se $\left\{x_{n}\right\}_{M \leqslant n \leqslant N}$ for uma hipersequência interna de ${ }^{*} X_{\omega}$-objetos com $M, N \in{ }^{*} \mathbb{Z}$, então o conjunto $\left\{x_{n}\right\}_{M \leqslant n \leqslant N}$ será hiperfinito (Proposição 4.38, Letra $(c)$ ).

Nesta seção, denotaremos por Fin $(E, P)$ a $L_{\epsilon}\left(X_{\omega}\right)$-fórmula (Definição A.26 e Nota A.27)

$$
(\exists ! n \in \mathbb{N})(\exists S \in \mathcal{P}(\mathbb{N}))(\exists f \in P) \phi(E, n, S, f),
$$

onde $\phi(E, n, S, f)$ é a conjunção das $L_{\epsilon}\left(X_{\omega}\right)$-fórmulas

1. $(\forall x \in S) x \in \mathbb{N} ; \quad$ 4. $(\forall s \in S)(\exists ! e \in E)(s, e) \in f$;

2. $(\forall x \in \mathbb{N})(x \in S \longleftrightarrow x \leqslant n)$;

3. $(\forall p \in f)(\exists s \in S)(\exists e \in E) p=(s, e) ; \quad$ 5. $(\forall e \in E)(\exists ! s \in S)(s, e) \in f$.

Se $A$ e $B$ forem dois $X_{\omega}$-conjuntos, então a condição $\models$ Fin $(A, \mathcal{P}(\mathbb{N} \times B))$ será verdadeira se, e somente se, $A$ for um subconjunto finito de $B$.

Teorema 4.40. Um ${ }^{*} X_{\omega}$-conjunto $A$ será hiperfinito se, e somente se, existir um único número hiperinteiro não negativo $n$ tal que existe uma bijeção interna $f:[1, n]_{*_{\mathbb{N}_{0}}} \rightarrow A$. 
Demonstração. O resultado é imediato para $A=\emptyset$, visto que a bijeção nula $\emptyset: \emptyset \rightarrow \emptyset$ é interna.

Suponha que $A$ é hiperfinito e não nulo. Assim, existirá um $X_{\omega}$-conjunto $F$ tal que $A \in{ }^{*} \mathcal{P}_{\omega}(F)$, e, como

$$
\models\left(\forall E \in \mathcal{P}_{\omega}(F)\right) \operatorname{Fin}(E, \mathcal{P}(\mathbb{N} \times F))
$$

pelo PT temos que existem exatamente um número hipernatural $n$, um

$$
S \in{ }^{*} \mathcal{P}(\mathbb{N}) \subset \mathcal{P}\left({ }^{*} \mathbb{N}\right)
$$

e uma função

$$
f \in{ }^{*} \mathcal{P}(\mathbb{N} \times F) \subset \mathcal{P}\left({ }^{*} \mathbb{N}_{0} \times{ }^{*} F\right)
$$

tais que $S=[1, n]_{* \mathbb{N}}$ e $f$ é uma bijeção do tipo $S \rightarrow A$, provando a condição necessária.

Suponha que existe um único número hipernatural $n$ tal que existe uma bijeção interna $f:[1, n]_{* \mathbb{N}} \rightarrow A$. Assim, existe um número natural $k$ com $A \in \mathbb{I}^{k}$ (Teorema 4.25, Letra $(e))$, e, como

$$
\models\left(\forall E \in X^{k}\right)\left[\operatorname{Fin}\left(E, \mathcal{P}\left(\mathbb{N} \times X_{k-1}\right)\right) \longrightarrow E \in \mathcal{P}_{\omega}\left(X_{k-1}\right)\right]
$$

pelo PT temos $A \in{ }^{*} \mathcal{P}_{\omega}\left(X_{k-1}\right)$, resultando que $A$ é hiperfinito.

Corolário 4.41. Seja E um $X_{\omega}$-conjunto.

(a) ${ }^{*}$ E será hiperfinito se, e somente se, E for finito;

(b) $U m^{*} X_{\omega}$-conjunto finito será hiperfinito se, e somente se, ele for interno.

Demonstração.

(a) Se $E$ for finito, então ${ }^{*} E$ será finito e será hiperfinito (Teorema 4.32, Letra $(a)$ ). Se ${ }^{*} E$ for hiperfinito, se $n$ for um número natural com $E \in X^{n}$, e se $E$ for infinito, então teremos

$$
\models \neg \operatorname{Fin}\left(E, \mathcal{P}\left(\mathbb{N} \times X_{n-1}\right)\right),
$$

o que é absurdo pelo PT.

(b) Suponha que $A$ é um ${ }^{*} X_{\omega}$-conjunto finito e interno. Como $A$ é finito, existe um

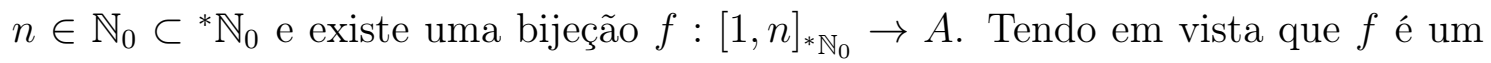
conjunto finito de pares ordenados internos (Teorema 4.32, Letras $(a)$ e $(b)$ ), temos que $f$ é interna, implicando que $A$ é hiperfinito. 
Exemplo 4.42. O conjunto dos números hipernaturais, * $\mathbb{N}$, não é hiperfinito. De fato, a *-transformação de qualquer $X_{\omega}$-conjunto infinito não é hiperfinita (Corolário 4.41, Letra $(a))$.

Definição 4.43. Se $A$ for um ${ }^{*} X_{\omega}$-conjunto hiperfinito, então o único número hipernatural $n$ tal que existe uma bijeção interna $f:[1, n]_{*_{\mathbb{N}_{0}}} \rightarrow A$ é chamado de cardinal interno de $A$ e é denotado por $\llbracket A \rrbracket$.

Exemplo 4.44. Temos $\llbracket[1, n]_{*_{\mathbb{N}}} \rrbracket=n\left(\forall n \in{ }^{*} \mathbb{N}_{0}\right)$, e, em particular, $\llbracket \emptyset \rrbracket=0$. Se $m$ e $n$ forem dois números hiperinteiros com $m \leqslant n$, então teremos $\llbracket[m, n]_{* \mathbb{Z}} \rrbracket=n-m+1$, visto que a função

$$
f:[1, n-m+1]_{* \mathbb{N}_{0}} \rightarrow[m, n]_{* \mathbb{Z}}
$$

dada por $f(x):=x+m-1$ é uma bijeção interna.

Proposição 4.45. Se $E$ for um $X_{\omega}$-conjunto, e se $f: \mathcal{P}_{\omega}(E) \rightarrow \mathbb{N}_{0}$ for a função dada por $f(A):=|A|$, então ${ }^{*} f$ será a função do tipo $\mathcal{P}_{h f}\left({ }^{*} E\right) \rightarrow{ }^{*} \mathbb{N}_{0}$ dada por ${ }^{*} f(A)=\llbracket A \rrbracket$.

Demonstração. Como $\models(\emptyset, 0) \in f$ e

$$
\models\left(\forall A \in \mathcal{P}_{\omega}(E)\right)(\forall n \in \mathbb{N})(\forall g \in \mathcal{P}(\mathbb{N} \times E))(\forall S \in \mathcal{P}(\mathbb{N}))(\phi(A, n, S, g) \longrightarrow(A, n) \in f),
$$

pelo PT temos que se $A \in{ }^{*}\left(\mathcal{P}_{\omega}(E)\right)=\mathcal{P}_{h f}\left({ }^{*} E\right)$, então $(A, \llbracket A \rrbracket) \in{ }^{*} f$.

A seguir, utilizaremos os conceitos desenvolvidos nesta seção para fornecer uma demonstração alternativa para o Teorema do Valor Intermediário, um dos resultados mais importantes da Análise Real. Nesse caso, devemos escolher qualquer conjunto $X$ que contenha $\mathbb{R}$.

Teorema 4.46 (Teorema do Valor Intermediário). Sejam $a, b \in \mathbb{R}$ com $a<b$. Se uma função $f:[a, b]_{\mathbb{R}} \rightarrow \mathbb{R}$ for contínua com $f(a) \neq f(b)$, e se y for um número real entre $f(a)$ e $f(b)$, então existirá um número real c entre a e b tal que $f(c)=y$.

Demonstração. Assumiremos que $f(a)<f(b)$. Seja $N$ um número hipernatural infinito, e seja $\left\{x_{n}\right\}_{0 \leqslant n \leqslant N}$ a hipersequência de números hiper-reais definida por

$$
x_{n}:=a+\frac{b-a}{N} \cdot n .
$$

Como o intervalo $[0, N]_{* \mathbb{N}}$ é interno (Exemplo 2.28), a sequência dos $x_{n}$ é interna (Corolário 2.29). Seja $A$ o conjunto não nulo dado por

$$
A:=\left\{x_{n}: 0 \leqslant n \leqslant N \mathrm{e}^{*} f\left(x_{n}\right)<y\right\} .
$$

Pelo PDI temos que $A$ é interno, e, como $A$ é um subconjunto do conjunto hiperfinito $\left\{x_{n}\right\}_{0 \leqslant n \leqslant N}$, temos que $A$ é hiperfinito (Proposição 4.38, Letras (b) e $(c)$ ). Como todo 
subconjunto finito não nulo de $\mathbb{R}$ tem um maior elemento, pelo $\mathrm{PT}$ temos que todo subconjunto hiperfinito não nulo de ${ }^{*} \mathbb{R}$ tem um maior elemento, implicando que existe um $k \in[0, N]_{* \mathbb{N}}$ tal que $x_{k}$ é o maior elemento de $A$. Seja $c:=\operatorname{st}\left(x_{k}\right) \in[a, b]_{\mathbb{R}}$. Temos

$$
{ }^{*} f\left(x_{k}\right)<y \leqslant{ }^{*} f\left(x_{k+1}\right)={ }^{*} f\left(x_{k}+\frac{b-a}{N}\right),
$$

e, como $f$ é contínua em $c$, temos (Teorema 2.41, Letra $(k)$ )

$$
{ }^{*} f\left(x_{k}\right) \sim f(c) \sim{ }^{*} f\left(x_{k}+\frac{b-a}{N}\right),
$$

resultando em $f(c) \sim y$ e $f(c)=y$ (Proposição 1.79).

\subsection{Operações sobre Hipersequências}

Na Seção 2.7, verificamos que a *-transformação da adição entre os números reais, ${ }^{*}+$, é uma extensão de + que pode ser vista como uma adição entre os números hiper-reais, e, na Seção 2.11, constatamos que essa operação pode ser estendida para obter somas de hipersequências internas de números hiper-reais. Nesta seção, veremos que qualquer lei de composição interna, assim como adição standard ${ }^{*}+$ em ${ }^{*} \mathbb{R}$, pode ser estendida para obter composições de hipersequências internas.

Consideraremos $\mathrm{um}^{*} X_{\omega}$-conjunto interno $A$, uma lei de composição interna $\oplus: A \times A \rightarrow A$, e um número natural $k$ com $\mathbb{Z},<\in X^{k}$ e $A, \oplus \in \mathbb{I}^{k}$, onde $<$ é a ordem usual em $\mathbb{Z}$.

Se $E$ for um $X_{\omega}$-conjunto de nível $k$, então cada lei de composição $f: E \times E \rightarrow E$ em $E$ terá nível $k+4$ em $X^{\omega}$, cada sequência $\left\{x_{i}\right\}_{m \leqslant i \leqslant n} \in{ }^{\operatorname{Int}(\mathbb{Z},<)} E$ terá nível $k+2$ em $X^{\omega}$, e cada função do tipo ${ }^{\operatorname{Int}(\mathbb{Z},<)} E-\{\emptyset\} \rightarrow E$ terá nível $k+5$ em $X^{\omega}$ (Teorema 4.8). Assim, o processo recursivo efetuado na Seção 2.11 com o intuito de definir a função $\mathcal{S}$ atesta a veridicidade da condição

$$
\models\left(\forall E \in X^{k}\right)\left(\forall f \in X^{k+4}\right)\left(f: E \times E \rightarrow E \longrightarrow\left(\exists g \in X^{k+5}\right) \psi(E, f, g)\right),
$$

onde $\psi(E, f, g)$ é a conjunção das $L_{\epsilon}\left(X_{\omega}\right)$-fórmulas:

- $g:{ }^{\operatorname{Int}(\mathbb{Z},<)} E-\{\emptyset\} \rightarrow E$;

- $\left(\forall x \in X^{k+2}\right)(\forall m \in \mathbb{Z})(x:\{m\} \rightarrow E \longrightarrow g(x)=x(m))$;

- $\left(\forall x, y \in X^{k+2}\right)(\forall m, n \in \mathbb{Z})\left[\left(m<n \wedge x:[m, n]_{\mathbb{Z}} \rightarrow E \wedge y:[m, n)_{\mathbb{Z}} \rightarrow E \wedge\right.\right.$

$\left.(\forall i \in \mathbb{Z})(m \leqslant i \leqslant n-1 \longrightarrow x(i)=y(i))) \longrightarrow g(x)=g(y) \oplus x_{n}\right]$.

Assim, pelo PT, existe uma função interna

$$
\bigoplus: \mathbb{I}^{\omega} \cap\left({ }^{\operatorname{Int}\left({ }^{*} \mathbb{Z},<\right)} A-\{\emptyset\}\right) \rightarrow A
$$

de nível $k+5$ em $\mathbb{I}^{\omega}$ tal que 
- $\bigoplus\left(\left\{x_{i}\right\}_{m \leqslant i \leqslant m}\right)=x_{m}$ para qualquer hipersequência interna $\left\{x_{i}\right\}_{m \leqslant i \leqslant m} \operatorname{com} x_{m} \in A$ cujo conjunto de índices é unitário;

- $\bigoplus\left(\left\{x_{i}\right\}_{m \leqslant i \leqslant n}\right)=\bigoplus\left(\left\{x_{i}\right\}_{m \leqslant i \leqslant n-1}\right) \oplus x_{n}$ para qualquer hipersequência interna $\left\{x_{i}\right\}_{m \leqslant i \leqslant n}$ de elementos de $A$ com $m, n \in * \mathbb{Z}$ e $m<n$.

Dizemos que a função $\bigoplus$ é a operação hiperfinita induzida por $\oplus$, visto que cada elemento do seu domínio define um conjunto hiperfinito (Proposição 4.38, Letra $(c)$ ). Caso a lei de composição $\oplus$ tenha um elemento neutro $0_{A} \in A$, a função $\bigoplus$ será estendida para o domínio $\mathbb{I}^{\omega} \cap \operatorname{Int}^{\left({ }^{*} \mathbb{Z},<\right)} A$ de modo que $\bigoplus(\emptyset):=0_{A}$.

Exemplo 4.47. Na Seção 2.11, lidamos com o caso particular $\oplus={ }^{*}+:{ }^{*} \mathbb{R} \times{ }^{*} \mathbb{R} \rightarrow{ }^{*} \mathbb{R}$. Naquela ocasião, definimos a função $\mathcal{S}: \operatorname{Int}(\mathbb{Z},<) \mathbb{R} \rightarrow \mathbb{R}$, a qual efetua somas finitas de sequências de números reais, e obtemos a sua $*$-transformação, ${ }^{*} \mathcal{S}: \mathbb{I}^{\omega} \cap \operatorname{Int}\left({ }^{*} \mathbb{Z},<\right)\left({ }^{*} \mathbb{R}\right) \rightarrow{ }^{*} \mathbb{R}$, a qual efetua somas hiperfinitas de hipersequências de números hiper-reais. Por indução interna (Exemplo 2.33) sobre o número hipernatural de elementos das hipersequências internas em $\operatorname{Int}\left({ }^{*} \mathbb{Z},<\right)\left({ }^{*} \mathbb{R}\right)$, obtém-se que ${ }^{*} \mathcal{S}$ é a função $\bigoplus$ definida nesta seção.

Exemplo 4.48. Se $A={ }^{*} \mathbb{R}$ e se $\oplus$ é a $*$-transformação da operação de multiplicação em $\mathbb{R}$, então a função $\bigoplus: \mathbb{I}^{\omega} \cap \operatorname{Int}\left({ }^{*} \mathbb{Z},<\right)\left({ }^{*} \mathbb{R}\right) \rightarrow{ }^{*} \mathbb{R}$ definida nesta seção é denotada por $\prod$ por abuso de linguagem. Se $x_{m} x_{m+1} \ldots x_{n}$ é uma hipersequência interna de números hiper-reais, então a imagem $\prod\left(\left\{x_{i}\right\}_{m \leqslant i \leqslant n}\right)$ é chamada de produto hiperfinito da hipersequência interna $x_{m} x_{m+1} \ldots x_{n}$, e, por abuso de linguagem, ela é denotada por $\prod_{i=m}^{n} x_{i}$ ou por

$$
x_{m} \cdot x_{m+1} \cdots x_{n} .
$$

Analogamente à demonstração do Teorema 2.53, prova-se que os produtos hiperfinitos satisfazem às seguintes propriedades:
(a) $\prod_{i=m}^{n} r \cdot x_{i}=r^{n} \cdot \prod_{i=m}^{n} x_{i}$
(b) $\left(\prod_{i=m}^{n} x_{i}\right) \cdot\left(\prod_{i=n+1}^{p} x_{i}\right)=\prod_{i=m}^{p} x_{i}$;
(c) $\prod_{i=m}^{n} x_{i}=\prod_{i=m+j}^{n+j} x_{i-j}$;
(d) $\prod_{i=m}^{n} x_{i} \leqslant \prod_{i=m}^{n} y_{i}$ se $0 \leqslant x_{i} \leqslant y_{i}\left(\forall i \in[m, n]_{* \mathbb{Z}}\right)$;
(e) $\left|\prod_{i=m}^{n} x_{i}\right|=\prod_{i=m}^{n}\left|x_{i}\right|$.

(Fatoração)

(Aditividade)

(Invariância Translacional)

(Monotonicidade)

(Distributividade de $|\cdot|)$ 
onde $x_{m} \ldots x_{n} \ldots x_{p}$ e $y_{m} \ldots y_{n}$ são duas hipersequências internas de números hiperreais com $m \leqslant n<p, r \in{ }^{*} \mathbb{R}$, e $j \in{ }^{*} \mathbb{Z}$.

Exemplo 4.49. Seja $E \in X^{n} \operatorname{com} n<\omega$, e seja $\cup: \mathcal{P}(E) \times \mathcal{P}(E) \rightarrow \mathcal{P}(E)$ a lei de composição em $\mathcal{P}(E)$ que associa cada par $(M, N)$ de subconjuntos de $E$ à união $M \cup N$. Assim, temos

$$
\models(\forall M, N \in \mathcal{P}(E))[M \cup N \subset E \wedge(\forall x \in E)(x \in M \cup N \leftrightarrow(x \in M \vee x \in N))]
$$

e, pelo PT, temos que

$$
{ }^{*} \cup:\left(\mathbb{I}^{\omega} \cap \mathcal{P}\left({ }^{*} E\right)\right) \times\left(\mathbb{I}^{\omega} \cap \mathcal{P}\left({ }^{*} E\right)\right) \rightarrow\left(\mathbb{I}^{\omega} \cap \mathcal{P}\left({ }^{*} E\right)\right)
$$

é a função dada por ${ }^{*} \cup(M, N)=M \cup N$, ou seja, a função ${ }^{*} \cup$ é essencialmente uma união. Analogamente, tomando $\oplus={ }^{*} \cup$ prova-se que $\bigoplus_{i=m}^{n} M_{i}=\bigcup_{i=m}^{n} M_{i}$ para qualquer hipersequência interna $\left\{M_{i}\right\}_{m \leqslant i \leqslant n}$ de subconjuntos internos de ${ }^{*} E$, implicando que a união $\bigcup_{i=m}^{n} M_{i}$ é interna. Esses resultados também são válidos ao considerarmos as interseções no lugar das uniões.

Exemplo 4.50. Considere a função mdc $: \mathbb{N} \times \mathbb{N} \rightarrow \mathbb{N}$, a qual associa cada par $(p, q)$ de números naturais ao maior divisor comum de $p$ e $q$. Temos

$$
\models(\forall p, q \in \mathbb{N})[\operatorname{mdc}(p, q)|p \wedge \operatorname{mdc}(p, q)| q \wedge(\forall n \in \mathbb{N})((n|p \wedge n| q) \rightarrow n \mid \operatorname{mdc}(p, q))],
$$

onde as $L_{\epsilon}\left(X_{\omega}\right)$-fórmulas da forma $a \mid b$ são dadas por $(\exists c \in \mathbb{N}) a \cdot c=b$, resultando pelo PT que se $p$ e $q$ forem dois números hipernaturais, então o número ${ }^{*}$ mdc $(p, q)$ será o maior divisor comum de $p$ e $q$ em ${ }^{*} \mathbb{N}$. Analogamente, tomando $\oplus={ }^{*}$ mdc prova-se que se $\left\{p_{i}\right\}_{m \leqslant i \leqslant n}$ é uma hipersequência interna de números hipernaturais, então a imagem $\bigoplus_{i=m}^{n} p_{i}$ é o maior divisor comum dos números $p_{i} \mathrm{em}{ }^{*} \mathbb{N}$. Esses resultados também são válidos ao considerarmos os menores múltiplos comuns no lugar dos maiores divisores comuns.

\subsection{Overflow e Underflow}

Concluiremos este capítulo com dois resultados que atuam como princípios poderosos na Teoria dos Monomorfismos Não Standard. Foi opção do autor não utilizar tais noções no Capítulo 2 e no restante deste capítulo, enfatizando, assim, a aplicabilidade direta do Princípio da Transferência nesta dissertação.

Teorema 4.51. Seja $\phi\left(x, y_{1} \ldots y_{n}\right)$ uma $L_{\epsilon}$-fórmula com quantificadores limitados, sejam $a_{1} \ldots a_{n} \in \mathbb{I}_{\omega}$, e seja $A$ o conjunto de números hipernaturais dado por

$$
A:=\left\{n \in{ }^{*} \mathbb{N}: * \models \phi\left(n, a_{1} \ldots a_{n}\right)\right\} .
$$


(a) (Princípio do Overflow) Se existir um $k \in \mathbb{N}$ tal que $[k, \infty)_{\mathbb{N}} \subset A$, então existirá um $K \in{ }^{*} \mathbb{N}-\mathbb{N}$ tal que $[k, K]_{* \mathbb{N}} \subset A$

(b) (Princípio do Underflow) Se existir um $K \in{ }^{*} \mathbb{N}-\mathbb{N}$ tal que $[1, K]_{*_{\mathbb{N}}}-\mathbb{N} \subset A$, então existirá um $k \in \mathbb{N}$ tal que $[k, K]_{* \mathbb{N}} \subset A$.

Demonstração. Pelo PDI, o conjunto $A$ é interno, assim como qualquer intervalo de números hipernaturais (Exemplo 2.28).

(a) A união $B:=[1, k-1]_{\mathbb{N}} \cup A$ é interna (Teorema 4.25, Letra $(c)$ ), e, pela hipótese, temos $\mathbb{N} \subset B \subset{ }^{*} \mathbb{N}$. Se $B={ }^{*} \mathbb{N}$, então $[k, \infty)_{*^{*}} \subset A$, e, em particular, teremos $[k, K]_{*_{\mathbb{N}}} \subset A$ para qualquer $K \in{ }^{*} \mathbb{N}-\mathbb{N}$. Se $B \neq{ }^{*} \mathbb{N}$, então a diferença interna *N $-B$ será não nula e terá um menor elemento $H$ (Exemplo 2.32) pertencente a $* \mathbb{N}-\mathbb{N}$, implicando em

$$
[k, H-1]_{* \mathbb{N}} \subset[k, \infty)_{* \mathbb{N}} \cap B \subset A .
$$

(b) A união $C:=[K+1, \infty)_{*_{\mathbb{N}}} \cup A$ é interna (Teorema 4.25, Letra $(c)$ ), e, pela hipótese, temos ${ }^{*} \mathbb{N}-\mathbb{N} \subset C \subset{ }^{*} \mathbb{N}$. Se $C={ }^{*} \mathbb{N}$, então $[1, K]_{* \mathbb{N}} \subset A$. Se $C \neq{ }^{*} \mathbb{N}$, então a diferença interna * $\mathbb{N}-C$ será não nula e terá um maior elemento $h$ (Exemplo 2.32) pertencente a $\mathbb{N}$, implicando em

$$
[h+1, K]_{*_{\mathbb{N}}} \subset[1, K]_{*_{\mathbb{N}}} \cap C \subset A .
$$

Se $S$ for um subconjunto interno de ${ }^{*} \mathbb{N}$, então, tomando $\phi\left(x, y_{1}\right):=x \in y_{1}$ e $a_{1}:=S$, teremos que o conjunto $A$ definido no enunciado acima é igual a $S$. É comum que os resultados do Teorema 4.51 sejam aplicados nessas circunstâncias.

Tratamos dos Princípios do Overflow e Underflow no caso dos conjuntos de números hipernaturais, mas existem múltiplas variações desses princípios relacionadas aos subconjuntos de ${ }^{*} \mathbb{Q}$ e $* \mathbb{R}$, as quais não serão abordadas neste trabalho.

Exemplo 4.52. Se $g:{ }^{*} \mathbb{N} \rightarrow{ }^{*} \mathbb{R}$ for uma função interna tal que $g(n) \sim 0(\forall n \in \mathbb{N})$, então todo número natural pertencerá ao conjunto

$$
A:=\left\{n \in{ }^{*} \mathbb{N}:|g(n)|<\frac{1}{n}\right\}
$$

e, pelo Princípio do Overflow, existirá um número hipernatural infinito $N$ tal que $|g(n)|<1 / n\left(\forall n \in[1, N]_{*_{\mathbb{N}}}\right)$, implicando, em particular, em $g(n) \sim 0\left(\forall n \in[1, N]_{*_{\mathbb{N}}}\right)$. Esse resultado é conhecido como Lema Sequencial de Robinson. 
Exemplo 4.53. Seja $S$ um subconjunto interno de * $\mathbb{N}$. Provaremos que $S$ conterá números hipernaturais infinitos arbitrariamente pequenos se, e somente se, $S$ contiver números naturais arbitrariamente grandes. Se a interseção $S \cap \mathbb{N}$ tiver um número natural $n$ como cota superior, então, como o complemento ${ }^{*} \mathbb{N}-S$ é interno e contém todo número no intervalo $[n+1, \infty)_{\mathbb{N}}$, pelo Princípio do Overflow existirá um número hipernatural infinito $K$ tal que $[n+1, K]_{*_{\mathbb{N}}} \subset{ }^{*} \mathbb{N}-S$, resultando em $S_{\infty} \subset[K+1, \infty)_{*_{\mathbb{N}}}$ e provando a implicação contrapositiva da condição necessária do problema. Reciprocamente, se a interseção $S \cap{ }^{*} \mathbb{N}_{\infty}$ tiver uma cota inferior $N$ em ${ }^{*} \mathbb{N}_{\infty}$, então a diferença $[1, N-1]_{* \mathbb{N}}-\mathbb{N}$ estará contida no conjunto interno ${ }^{*} \mathbb{N}-S$, e, pelo Princípio do Underflow, existirá um número natural $k$ tal que $[k, N-1]_{*_{\mathbb{N}}} \subset{ }^{*} \mathbb{N}-S$. Nesse caso, teremos $S \cap \mathbb{N} \subset[1, k-1]_{\mathbb{N}}$ e o conjunto $S \cap \mathbb{N}$ será finito, finalizando a demonstração da equivalência desejada. 


\section{5}

\section{Monomorfismos Não Standard Existem}

Os resultados dos Capítulos 2 e 4 não têm relevância matemática até que a existência dos monomorfismos não standard $*: X_{\omega} \rightarrow Y_{\omega}$ seja demonstrada em NBG. Veremos neste capítulo que o Axioma da Escolha e o aparato conceitual desenvolvido no Capítulo 3 e na Seção 4.1 são suficientes para descrever uma construção de uma função com as propriedades desejadas. Essa idealização de *, concebida por Abraham Robinson e Elias Zakon $(46,54)$, é parcialmente ardilosa e emaranhada, embora seja a mais simples conhecida. A fim de elucidá-la, procederemos, inicialmente, à descrição de uma função $\star: X_{\omega} \rightarrow Y_{\omega}$, para depois definirmos a função $*$ pretendida evitando um empecilho peculiar que impede que $\star$ seja um monomorfismo não standard.

Assim como fizemos na Seção 3.8, aproveitaremos a imersão canônica associada a uma ultrapotência $\prod_{\mathcal{U}} X$ para iniciar a concepção de $*$, onde será necessário que $\mathcal{U}$ seja um ultrafiltro não principal em um conjunto $I$. Como existe um modelo de NBG $-\{A C\}$ no qual todo ultrafiltro em todo conjunto $I$ é principal, deduz-se que o Axioma da Escolha é indispensável para que a construção descrita neste capítulo seja possível. De fato, não é conhecida uma única maneira de obter * sem a utilização do Axioma da Escolha.

\subsection{Cópias Base}

Verificamos que há diversas vantagens em assumirmos que os conjuntos $X$ e $Y$ são conjuntos base ao lidarmos com um monomorfismo não standard $*: X_{\omega} \rightarrow Y_{\omega}$. Nesta seção, veremos que essa imposição não limita a escolha de $X$, contanto que tal conjunto seja identificado com uma cópia dele. Também constataremos que as potências reduzidas da forma $\prod_{\mathcal{F}} X$ são conjuntos base em certas circustâncias, e isso será suficiente para assegurarmos que o conjunto $Y$ seja um conjunto base na construção de $*$ apresentada no restante deste capítulo.

Lema 5.1. Seja $\alpha$ um ordinal infinito. Se $X$ for um conjunto tal que $\emptyset \notin X$ e todo elemento de um elemento de $X$ tem posto $\alpha$, então $X$ será um conjunto base.

Demonstração. Provaremos por indução sobre o número inteiro não negativo $n$ que cada $X_{\omega}$-objeto $x$ de nível $n$ é tal que posto $(x)<n$ ou $\alpha<\operatorname{posto}(x) \leqslant \alpha+n+1$. Tal afirmação é válida para $n=0$, visto que todo $X_{\omega}$-átomo tem posto $\alpha+1$. Suponha que ela é válida para um número $n$ e seja $x \in X_{n+1}$. Se $x \in X$, então posto $(x)=\alpha+1 \leqslant \alpha+(n+1)+1$. Considere o caso em que $x \in X^{n+1}$, ou seja, $x \subset X_{n}$. Assim, pela hipótese de indução, todo elemento de $x$ terá posto menor que $n$ ou pertencente ao intervalo $(\alpha, \alpha+n+1]_{\text {On }}$. 
Se todo elemento de $x$ tiver posto menor que $n$, então o posto de $x$ será menor que $n+1$ (Proposição B.33), e se $x$ tiver pelo menos um elemento no intervalo $(\alpha, \alpha+n+1]_{\text {On }}$, então, como $\alpha$ é infinito, teremos $\alpha<\operatorname{posto}(x) \leqslant \alpha+(n+1)+1$, finalizando a indução.

Portanto, se $a \in X$ e $x \in a \cap X_{\omega}$, então $x$ terá posto $\alpha$ e existirá um número inteiro não negativo $n$ tal que $x \in X_{n}$, resultando em posto $(x)<n<\alpha$ ou $\alpha<\operatorname{posto}(x) \leqslant \alpha+n+1$, o que é absurdo.

Se $x$ e $y$ forem quaisquer dois objetos, então o posto do par ordenado $(x, y)$ será dado por

$$
\begin{aligned}
\operatorname{posto}((x, y)) & =\operatorname{posto}(\{\{x\},\{x, y\}\}) \\
& =\max \{\operatorname{posto}(\{x\}), \operatorname{posto}(\{x, y\})\}+1 \\
& =\max \{\operatorname{posto}(x)+1, \max \{\operatorname{posto}(x), \operatorname{posto}(y)\}+1\}+1 \\
& =\max \{\operatorname{posto}(x), \operatorname{posto}(y)\}+2 .
\end{aligned}
$$

Portanto, o posto de um par ordenado é duas unidades maior que o maior posto das suas coordenadas.

Proposição 5.2 (Cópias Base). Seja $X$ um conjunto. Existe um conjunto $X^{\prime}$ equipotente a $X$ que é um conjunto base.

Demonstração. Seja $\alpha$ um ordinal infinito maior ou igual ao posto de $X$, e seja $X^{\prime}$ o conjunto

$$
X^{\prime}:=\{\{(x, \alpha)\}: x \in X\} .
$$

Note que $X^{\prime}$ é equipotente a $X$. Se $x \in X$, então posto $((x, \alpha))=\alpha+2$, visto que

$$
\operatorname{posto}(x)<\operatorname{posto}(X) \leqslant \alpha=\text { posto }(\alpha)
$$

Todo elemento de um elemento de $X^{\prime}$ tem posto infinito e igual a $\alpha+2$, resultando que $X^{\prime}$ é um conjunto base pelo Lema 5.1.

Vale lembrar que se $X^{\prime}$ for uma cópia de $X$, então qualquer estrutura matemática relacionada ao conjunto $X$ pode ser convertida em uma estrutura equivalente relacionada a $X^{\prime}$. Por exemplo, se $f: X \rightarrow X^{\prime}$ for uma bijeção, e se $R$ for uma relação $n$-ária em $X$, então a relação $n$-ária $R^{\prime}$ em $X^{\prime}$ definida pela equivalência

$$
\left(x_{1} \ldots x_{n}\right) \in R^{\prime} \Leftrightarrow\left(f^{-1}\left(x_{1}\right) \ldots f^{-1}\left(x_{n}\right)\right) \in R
$$

será essencialmente igual à relação $R$. 
Proposição 5.3 (Potências Reduzidas Base). Seja X um conjunto, seja I um conjunto não nulo de posto infinito, e seja $\mathcal{F}$ um filtro em $I$. Se

$$
\text { posto }(X) \leqslant \operatorname{posto}(I),
$$

então a potência reduzida $\prod_{\mathcal{F}} X$ será um conjunto base.

Demonstração. Se $i \in I$, então posto $(i)<\operatorname{posto}(I)$, e se $x \in X$, então

$$
\text { posto }(x)<\operatorname{posto}(X) \leqslant \operatorname{posto}(I) \text {. }
$$

Seja $f: I \rightarrow X$ uma função. Se posto $(I)$ for um ordinal sucessor, então existirá um ordinal $\alpha$ com posto $(I)=\alpha+1$ tal que posto $(i) \leqslant \alpha(\forall i \in I)$, e existirá um elemento $i_{\alpha}$ de $I$ com posto $\left(i_{\alpha}\right)=\alpha$. Nesse caso, se $i \in I$, então os ordinais posto $(i)$ e posto $(f(i))$ serão menores ou iguais a $\alpha$ (Teorema B.16), implicando em posto $((i, f(i))) \leqslant \alpha+2(\forall i \in I)$ com a igualdade ocorrendo no caso $i=i_{\alpha}$, e posto $(f)=\alpha+3$.

Suponha que posto $(I)$ é um ordinal limite. Assim, se $(i, x) \in f$, então teremos (Teorema B.23)

$$
\text { posto }((i, x))=\max (\operatorname{posto}(i), \operatorname{posto}(x))+2<\operatorname{posto}(I)
$$

e se $\beta$ for qualquer ordinal menor que posto $(I)$, então existirá um $i_{\beta} \in I$ com $\beta<\operatorname{posto}\left(i_{\beta}\right)<\operatorname{posto}(I)$, resultando em

$$
\beta<\max \left(\operatorname{posto}\left(i_{\beta}\right), \operatorname{posto}\left(f\left(i_{\beta}\right)\right)\right)+2=\operatorname{posto}\left(\left(i_{\beta}, f\left(i_{\beta}\right)\right)\right)<\operatorname{posto}(I)
$$

e posto $(f)=\operatorname{posto}(I)$. Portanto, o posto de qualquer função $f: I \rightarrow X$ é infinito e não depende de $f$, provando que $\prod_{\mathcal{F}} X$ é um conjunto base pelo Lema 5.1.

\subsection{A Ideia da Construção de *}

Assumiremos um conjunto base $X$ como o único input da nossa discussão, e, ao decorrer deste capítulo, definiremos um conjunto base $Y$ e uma função $*: X_{\omega} \rightarrow Y_{\omega}$ que satisfaz às condições (Mon1)-(Mon3) da Definição 4.15:

(Mon1) ${ }^{*} X=Y$

(Mon2) Para todo subconjunto infinito $A$ de $X$, teremos $A \subsetneq{ }^{*} A$;

(Mon3) (Princípio da Transferência; PT) Para toda $L_{\epsilon}$-fórmula $\phi\left(x_{1} \ldots x_{n}\right)$ com quantificadores limitados, temos

$$
\left.\models \phi\left[a_{1} \ldots a_{n}\right] \Leftrightarrow * \models \phi{ }^{*} a_{1} \ldots{ }^{*} a_{n}\right]
$$

para quaisquer $a_{1} \ldots a_{n} \in X_{\omega}$ 
onde estamos aplicando a Notações 4.17 e 4.18.

Como vimos na Seção 3.8, é razoável definir $Y={ }^{*} X$ como uma ultrapotência da forma $\prod_{\mathcal{U}} X$. Por ora, escolheremos um conjunto não nulo $I$ qualquer e um ultrafiltro $\mathcal{U}$ qualquer em $I$, e definiremos:

Definição 5.4. Seja $Y$ a ultrapotência $\prod_{\mathcal{U}} X$.

A Proposição 5.3 nos fornece uma condição suficiente para que a ultrapotência $Y$ seja um conjunto base. Por tal razão, faremos a seguinte suposição:

Suposição 5.5. O conjunto I tem posto infinito e maior ou igual a posto $(X)$.

Exemplo 5.6. Se $J$ for um conjunto qualquer, então o conjunto

$$
I:=\{(i, \max (\omega, \operatorname{posto}(X))) \vdots i \in J\}
$$

é uma cópia de $J$ que tem posto infinito e maior que posto $(X)$.

Assim, $Y$ é um conjunto base.

A ideia da construção de $*$ consiste em obter uma sequência enumerável de funções $*_{0} *_{1} *_{2} \ldots$ tal que

$$
*_{0} \subset *_{1} \subset *_{2} \subset *_{3} \subset \cdots
$$

e $*_{n}: X_{n} \rightarrow Y_{n}$ para cada $n$, e a função $*$ desejada será a extensão infinita $\bigcup_{n=0}^{\infty} *_{n}$ de todas as funções dessa sequência. Portanto, se já tivermos definido a função $*_{n}$, então precisaremos determinar os valores da função $*_{n+1}$ apenas em argumentos pertencentes à diferença $X_{n+1}-X_{n}$, os quais são os $X_{\omega}$-objetos de posto $n$ em $X_{\omega}$ (Definição 4.9). Se $x \in X_{n}$, então $*_{n+1}(x):=*_{n}(x)$. para $*_{0}$.

A função canônica $X \rightarrow \prod_{\mathcal{U}} X$, a qual é dada por $x \mapsto \lim _{X, \mathcal{U}} x$, é uma escolha notória Definição 5.7. Seja $*_{0}: X_{0} \rightarrow Y_{0}$ a imersão elementar canônica relacionada à ultrapotência $Y=Y_{0}=\prod_{\mathcal{U}} X$.

Como definir a função $*_{1}: X_{1} \rightarrow Y_{1}$ ? Devemos prescrever os seus valores apenas em $X_{\omega}$-objetos de posto 0, ou seja, em subconjuntos $A$ de $X$. Vimos na Seção 3.8 que os limites integrais dos subconjuntos de $\mathbb{R}$ são adequados para indicar as *-transformações desses subconjuntos. Por essa razão, definiremos $*_{1}$ de maneira análoga, substituindo $\mathbb{R}$ por $X\left(\right.$ ou $\left.X_{0}\right)$. 
Definição 5.8. Para cada subconjunto $A$ de $X$, seja

$$
*_{1}(A):=\left\{\lim _{X, \mathcal{U}} s(i) \vdots s \in{ }^{I} X_{0} \text { e } s(i) \in A \text { q.t.p. }\right\} .
$$

Assim, temos

$$
\begin{aligned}
*_{1}(X) & =\left\{\lim _{X, \mathcal{U}} s(i) \vdots s \in{ }^{I} X_{0} \text { e } s(i) \in X \text { q.t.p. }\right\} \\
& =\left\{\lim _{X, \mathcal{U}} s(i) \vdots s \in{ }^{I} X\right\} \\
& =Y
\end{aligned}
$$

satisfazendo ao axioma (Mon1).

\subsection{Tentativa para Definir $*_{n}$}

O próximo passo é determinar as imagens da função $*_{2}$ nos elementos de $X_{2}$ de posto 1 , ou seja, nos subconjuntos $\mathcal{A}$ de $X_{1}$ que têm pelo menos um elemento de posto 0. A formulação dessa etapa é explicitada quando analisamos as definições de $*_{0}$ e $*_{1}$ e tentamos encontrar um padrão para a definição geral de $*_{n}$. Podemos fatorar $*_{0}$ em uma composição funcional $q_{0} \circ p_{0}$ conforme o esquema

$$
\begin{array}{cccccc}
x & \stackrel{p_{0}}{\mapsto} & \{x\}_{i \in I} & \stackrel{q_{0}}{\mapsto} & \lim _{X, \mathcal{U}} x \\
X_{0} & \stackrel{p_{0}}{\rightarrow} & { }^{I} X_{0} & \stackrel{q_{0}}{\rightarrow} & Y_{0}
\end{array}
$$

e podemos fatorar $*_{1}$ na forma $q_{1} \circ p_{1}$ conforme o esquema

$$
\begin{array}{cccc}
A & \stackrel{p_{1}}{\mapsto}\{A\}_{i \in I} \stackrel{q_{1}}{\mapsto}\left\{\left\{\begin{array}{ll}
q_{0}(A) & \text { se } A \in X_{0} \\
\left\{\lim _{X, \mathcal{U}} s(i) \vdots s \in{ }^{I} X_{0} \text { e } s(i) \in A \text { q.t.p. }\right\} & \text { se } A \in X_{1}-X_{0}
\end{array}\right.\right. \\
X_{1} \stackrel{p_{1}}{\rightarrow} \quad{ }^{I} X_{1} \quad \stackrel{q_{1}}{\rightarrow} & Y_{1}
\end{array}
$$

onde:

- $q_{0}:{ }^{I} X_{0} \rightarrow Y_{0}$ é dada por $q_{0}(r):=\lim _{X, \mathcal{U}} r(i)$;

- $q_{1}:{ }^{I} X_{1} \rightarrow Y_{1}$ é a extensão de $q_{0}$ tal que

$$
\begin{aligned}
q_{1}(r) & :=\left\{\lim _{X, \mathcal{U}} s(i) \vdots r \in{ }^{I} X_{0} \text { e } s(i) \in r(i) \text { q.t.p. }\right\} \\
& =\left\{q_{0}(s) \vdots s \in{ }^{I} X_{0} \text { e } s(i) \in r(i) \text { q.t.p. }\right\}
\end{aligned}
$$

para todo $r \in{ }^{I} X_{1}-{ }^{I} X_{0}$. 
As funções $p_{0}$ e $p_{1}$ exibem um padrão previsível.

Definição 5.9. Para cada $n<\omega$, seja $p_{n}$ a função $X_{n} \rightarrow{ }^{I} X_{n}$ dada por

$$
p_{n}(x):=\{x\}_{i \in I}
$$

A extensão infinita das funções $p_{n}$, a qual é dada pela união $\bigcup_{n=0}^{\infty} p_{n}$ e é uma função do tipo $X_{\omega} \rightarrow \bigcup_{n=0}^{\infty} X_{n}$, será denotada por $p$.

$\operatorname{Temos} p\left\lceil X_{n}=p_{n}(\forall n<\omega) \mathrm{e}\right.$

$$
p_{0} \subset p_{1} \subset p_{2} \subset \cdots .
$$

Os valores da função $q_{1}$ dependem exclusivamente de $q_{0}$. Exploraremos esse padrão recursivo para determinar as outras funções $q_{n}$.

\section{Definição 5.10 .}

- Supondo que a função $q_{n}$ já foi determinada, definimos a função $q_{n+1}$ como sendo a extensão da função $q_{n}$ ao domínio ${ }^{I} X_{n+1}$ tal que

$$
q_{n+1}(s):=\left\{q_{n}(r) \vdots r \in{ }^{I} X_{n} \text { e } r(i) \in s(i) \text { q.t.p. }\right\}
$$

para cada $s \in{ }^{I} X_{n+1}-{ }^{I} X_{n}$;

- Para cada $n<\omega$, seja $\star_{n}:=q_{n} \circ p_{n}$;

- A extensão infinita das funções $\star_{n}$, a qual é dada por $\bigcup_{n=0}^{\infty} \star_{n}$, será denotada por $\star_{\text {. }}$

Temos $\star_{0}=*_{0}, \star_{1}=*_{1}, \mathrm{e}$

$$
q_{0} \subset q_{1} \subset q_{2} \subset \cdots
$$

A verificação de que o conjunto $Y_{n}$ pode ser considerado como um contradomínio da função $q_{n}$ não é necessária para a discussão deste capítulo e será deixada para o leitor. Posto isso, cada $\star_{n}$ é do tipo $X_{n} \rightarrow Y_{n}$, e $\star$ é do tipo $X_{\omega} \rightarrow Y_{\omega}$.

No exemplo a seguir, mostraremos que a função $\star$ não é um monomorfismo não standard no caso $X \neq \emptyset$. Assim, não podemos definir as funções $*_{2} *_{3} *_{4} \ldots$ da maneira que definimos $\star_{2} \star_{3} \star_{4} \ldots$, e, como veremos na Seção 5.4, há uma maneira sutil de alterar a caracterização recursiva das funções $\star_{n}$ que é adequada para obter $*_{n}$. 


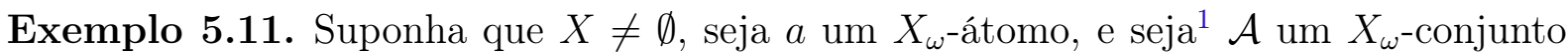
de posto 1 tal que $a \in \mathcal{A}$. Empregando a Notação 2.4 para a função $\star$, temos

$$
\begin{aligned}
{ }^{\star} \mathcal{A} & =q_{2}\left(p_{2}(\mathcal{A})\right) \\
& =q_{2}\left(\{\mathcal{A}\}_{i \in I}\right) \\
& =\left\{q_{1}(r) \vdots r \in{ }^{I} X_{1} \text { e } r(i) \in \mathcal{A} \text { q.t.p. }\right\} .
\end{aligned}
$$

Seja $Z \in \mathcal{U}$ e seja $r: I \rightarrow X_{1}$ a função dada por

$$
r(i):= \begin{cases}a & \text { se } i \in Z \\ \emptyset & \text { se } i \in I-Z\end{cases}
$$

Assim, temos $r \in{ }^{I} X_{1}$ e $r(i) \in \mathcal{A}$ q.t.p., resultando em

$$
\left\{q_{0}(t) \vdots t \in{ }^{I} X_{0} \text { e } t(i) \in r(i) \text { q.t.p. }\right\}=q_{1}(r) \in{ }^{\star} \mathcal{A}
$$

Se $t \in{ }^{I} X_{0}$ e $t(i) \in r(i)$ q.t.p., então, como $r(i) \in X_{0}$ q.t.p., teremos (Proposição 3.16, Letra $(c))$

$$
t(i) \in r(i) \in X_{0} \text { q.t.p., }
$$

o que é absurdo pois $X$ é um conjunto base. Isso prova que $\emptyset=q_{1}(r) \in{ }^{\star} \mathcal{A}$.

Se a função $\star: X_{\omega} \rightarrow Y_{\omega}$ for um monomorfismo não standard, então pelo Exemplo 4.31 teremos

$$
{ }^{\star} \emptyset=\emptyset \in{ }^{\star} \mathcal{A},
$$

implicando em $\emptyset \in \mathcal{A}$ pelo PT. Nesse caso, como $\mathcal{A}$ é arbitário, o conjunto vazio pertencerá a todo $X_{\omega}$-conjunto de posto 1 que tem um átomo como elemento, o que é claramente um absurdo como diversos contraexemplos mostram. Portanto, a função * indicada na Definição 5.10 não pode ser um monomorfismo não standard quando $X \neq \emptyset$.

\subsection{Outra Fatoração de $*_{n}$}

Investigando o Exemplo 5.11, observa-se que as inconveniências surgem quando aplicamos a função $q_{1}$ a uma função $r \in{ }^{I} X_{1}-{ }^{I} X_{0}$ tal que $r(i) \in X_{0}$ q.t.p. Caso pudéssemos aplicar a definição de $q_{0}$ ao calcularmos a imagem $q_{1}(r)$, ou seja, caso de alguma maneira pudéssemos considerar $r$ como um elemento de ${ }^{I} X_{0}=\operatorname{dom}\left(q_{0}\right)$, teríamos

$$
\begin{aligned}
q_{1}(r) & \text { " }=" q_{0}(r) \\
& ="=\lim _{X, \mathcal{U}} r(i)
\end{aligned}
$$

$\overline{1}$ Existe um $X_{\omega}$-conjunto com tal propriedade. Como exemplo, podemos tomar $\mathcal{A}=\{a,\{a\}\}$. 
em vez de $q_{1}(r)=\emptyset$, tornando a conclusão da argumentação daquele exemplo impraticável. Há coerência e significância em expressar o ultralimite $\lim _{X, \mathcal{U}} r(i)$ (Notação 3.21), e isso sugere que devemos considerar uma nova sequência de funções

$$
f_{0} \subset f_{1} \subset f_{2} \subset \cdots
$$

definidas de modo análogo às funções $q_{0} q_{1} q_{2} \ldots$ tal que os elementos do domínio de cada $f_{n}$ é o conjunto das funções $r: I \rightarrow X_{\omega}$ tais que $r(i) \in X_{n}$ q.t.p. Informalmente, pode-se interpretar dom $\left(f_{n}\right)$ como sendo o conjunto das funções do tipo $I \rightarrow X_{\omega}$ que "praticamente pertencem a ${ }^{I} X_{n}$ ".

\section{Definição 5.12 .}

- Para cada $n<\omega$, seja

$$
W_{n}:=\left\{r \in{ }^{I} X_{\omega}: r(i) \in X_{n} \text { q.t.p. }\right\}
$$

e seja $W_{\omega}:=\bigcup_{n=0}^{\infty} W_{n}$

- Seja $f_{0}: W_{0} \rightarrow Y_{0}$ a função dada por

$$
f_{0}(r):=\lim _{X, \mathcal{U}} r(i)
$$

onde tal ultralimite é determinado na Notação 3.21. Supondo que a função $f_{n}$ já foi determinada, definimos a função $f_{n+1}$ como sendo a extensão da função $f_{n}$ ao domínio $W_{n+1}$ tal que

$$
f_{n+1}(r):=\left\{f_{n}(s) \vdots s \in W_{n} \text { e } s(i) \in r(i) \text { q.t.p. }\right\}
$$

para cada $r \in W_{n+1}-W_{n}$

- A extensão infinita das funções $f_{n}$, a qual é dada por $\bigcup_{n=0}^{\infty} f_{n}$, será denotada por $f$.

Os níveis $Y_{n}$ em $Y_{\omega}$ podem ser considerados como contradomínios das funções $f_{n}$.

Proposição 5.13. Para cada ordinal finito $n$, temos $\operatorname{Im}\left(f_{n}\right) \subset Y_{n}$.

Demonstração. Claramente, a afirmação do enunciado é válida no caso $n=0$. Se ela for válida para todo ordinal menor ou igual a um ordinal finito $n$, e se $s \in W_{n+1}-W_{n}$, então

$$
f_{n+1}(s)=\left\{f_{n}(r) \vdots r \in W_{n} \text { e } r(i) \in s(i) \text { q.t.p. }\right\} \subset Y_{n}
$$

e $f_{n+1}(s) \in Y^{n+1}$, provando que $\operatorname{Im}\left(f_{n+1}\right) \subset Y_{n+1}$. Pelo Princípio da Indução, a demonstração está completa. 
Cada função $f_{n}$ é do tipo $W_{n} \rightarrow Y_{n}$, e $f$ é do tipo $W_{\omega} \rightarrow Y_{\omega}$.

Até o restante deste capítulo, aplicaremos as Proposições 3.16 e 3.40 sem mencioná-las explicitamente.

Proposição 5.14. Se $r \in{ }^{I} X_{\omega}$, se $s \in W_{n+1} \operatorname{com} n<\omega$, e se $r(i) \in s(i)$ q.t.p., então $s \notin W_{0}$ e $r \in W_{n}$.

Demonstração. Se $s \in W_{0}$, então $s(i) \in X$ q.t.p. e $r(i) \in s(i) \in X$ q.t.p., o que é absurdo pois $X$ é um conjunto base. Portanto, temos $s \notin W_{0}, s(i) \in X^{n+1}$ q.t.p. e $r(i) \in s(i) \subset X_{n}$ q.t.p., resultando em $r(i) \in X_{n}$ q.t.p.

Corolário 5.15. Para cada ordinal finito $n$ e para cada $s \in W_{n+1}$, temos

$$
\begin{aligned}
f(s) & =f_{n+1}(s) \\
& =\left\{f_{n}(r) \vdots r \in W_{\omega} \text { e } r(i) \in s(i) \text { q.t.p. }\right\} \\
& =\left\{f(r) \vdots r \in W_{\omega} \text { e } r(i) \in s(i) \text { q.t.p. }\right\} \\
& =\left\{f(r) \vdots r \in{ }^{I} X_{\omega} \text { e } r(i) \in s(i) \text { q.t.p. }\right\} .
\end{aligned}
$$

Proposição 5.16. Sejam $r, s \in W_{\omega}$.

(a) $\quad f(r)=f(s)$ se, e somente se, $r(i)=s(i)$ q.t.p.;

(b) $\quad f(r) \in f(s)$ se, e somente se, $r(i) \in s(i)$ q.t.p.

Demonstração. A letra (b) é consequência imediata da letra $(a)$.

(a) Se $r, s \in W_{0}$, então

$$
\lim _{X, \mathcal{U}} r(i)=f_{0}(r)=f(r)=f(s)=f_{0}(s)=\lim _{X, \mathcal{U}} s(i)
$$

e a conclusão desejada será imediata. Suponha que o resultado desejado é válido para os elementos de $W_{n}(n<\omega)$, suponha que $r, s \in W_{n+1}-W_{n}$, e suponha que $r(i) \neq s(i)$ q.t.p. Pelo Axioma da Extensão (Definições B.1 e B.4), temos

$$
(r(i) \subset s(i) \Rightarrow s(i) \not \subset r(i)) \text { q.t.p., }
$$

e podemos assumir que $r(i) \not \subset s(i)$ q.t.p. sem perda de generalidade. Como $r, s \notin W_{0}$, temos

$$
\left(r(i) \not \subset s(i) \wedge r(i), s(i) \in X^{\omega}\right) \text { q.t.p. }
$$

ou seja, existe um $U \in \mathcal{U}$ tal que $r(i) \not \subset s(i)$ e $r(i), s(i) \in X^{\omega}$ para todo $i \in U$. Para cada $i \in U$, seja $u(i)$ um elemento de $r(i)$ que não pertence a $s(i)$, e, para cada 
$i \in I-U$, seja $u(i):=\emptyset$. Definimos, assim, uma função $u: I \rightarrow X_{\omega}$ pertencente a $W_{n}$ (Proposição 5.14) tal que $u(i) \in r(i)$ q.t.p. e $u(i) \notin s(i)$ q.t.p., resultando em $f(u) \in f(r)$. Se $f(u) \in f(s)$, então existirá um $v \in W_{n} \operatorname{com} v(i) \in s(i)$ q.t.p. tal que $f(u)=f(v)$, e, pela hipótese de indução, teremos $u(i)=v(i)$ q.t.p. e $u(i) \in s(i)$ q.t.p., o que é absurdo. Portanto, temos $f(u) \in f(r)-f(s)$, o que é absurdo, provando a proposição pelo Princípio da Indução.

Enfim, dispomos das ferramentas necessárias para trabalhar com os objetos matemáticos desejados neste capítulo: as funções $*_{0} *_{1} *_{2} \ldots$ e a função $*$.

Definição 5.17. Para cada $n<\omega$, seja

$$
*_{n}:=f_{n} \circ p_{n}: X_{n} \rightarrow Y_{n}
$$

e seja

$$
*:=\bigcup_{n=0}^{\infty} *_{n}: X_{\omega} \rightarrow Y_{\omega} .
$$

Note que $*_{n}=* \uparrow_{X_{n}}(\forall n<\omega)$ e $*=f \circ p$.

Corolário 5.18. Para cada $X_{\omega}$-conjunto não nulo $A$, temos

$$
{ }^{*} A=\left\{f(r) \vdots r \in{ }^{I} A\right\} .
$$

Demonstração. Seja $n<\omega$ tal que $A \in X^{n}$, e seja a um elemento de $A$. Se $r: I \rightarrow A$ for uma função, então, como $A \subset X_{n-1}$ e $I \in \mathcal{U}$, teremos $r: I \rightarrow X_{\omega}$ e $r(i) \in A$ q.t.p., implicando que $f(r)$ pertencerá a ${ }^{*} A$. Suponha que $s: I \rightarrow X_{\omega}$ é uma função com $r(i) \in A$ q.t.p., seja $U:=s^{-1}\langle A\rangle$, e seja $r: I \rightarrow A$ a função dada por

$$
r(i):= \begin{cases}s(i) & \text { se } i \in U \\ a & \text { se } i \in I-U .\end{cases}
$$

Assim, temos $U \in \mathcal{U}, \quad s(i)=r(i)$ q.t.p., e $f(s)=f(r) \in\left\{f(r) \vdots r \in{ }^{I} A\right\}$ pela Proposição 5.16.

\subsection{Monomorfismos Não Standard Existem}

Com o acréscimo de uma imposição sobre as características do ultrafiltro $\mathcal{U}$ em $I$, provaremos que a função $*: X_{\omega} \rightarrow Y_{\omega}$ é um monomorfismo não standard, alicerçando, assim, a teoria acerca dessas funções descrita nos Capítulos 2 e 4. 
Se $A \subset X$, então

$$
\begin{aligned}
*\langle A\rangle & =\left\{{ }^{*} x \vdots x \in A\right\} \\
& =\left\{\lim _{X, \mathcal{U}} x \vdots x \in A\right\} \\
& \subset\left\{\lim _{X, \mathcal{U}} r(i) \vdots r \in W_{0} \text { e } r(i) \in A \text { q.t.p. }\right\} \\
& ={ }^{*} A,
\end{aligned}
$$

ou seja, $A \subset{ }^{*} A$ (Notação 4.18).

Ao lidarmos com o caso particular $X=\mathbb{R}$ e $I=\mathbb{N}$ na Seção 3.8, atestamos que é suficiente assumir que o ultrafiltro $\mathcal{U}$ em $I$ é $\omega_{1}$-incompleto para assegurar que os subconjuntos infinitos $A$ de $X$ estejam propriamente contidos em seus ultralimites integrais $\lim _{X, \mathcal{U}} A$ (Corolário 3.70). Como a definição da função $*_{1}=* \uparrow_{X_{1}}$ é fundamentada na noção de ultralimite integral (Definição 5.8), faremos essa mesma suposição a respeito do ultrafiltro $\mathcal{U}$ em $I$.

Suposição 5.19. O ultrafiltro $\mathcal{U}$ em I é $\omega_{1}$-incompleto.

Pelo Exemplo 3.55, o conjunto $I$ é infinito.

Observação 5.20. Pelo Teorema 3.58, existe um ultrafiltro $\mathcal{U}$ em $I$ que satisfaz à Suposição 5.19. Pelo Exemplo 5.6, podemos escolher o conjunto $I$ de modo que ele seja uma cópia de $\mathbb{N}$, e, nesse caso, o Lema do Ultrafiltro e a Proposição 3.57 implicam a existência de $\mathcal{U}$ com a propriedade desejada.

Proposição 5.21. A função $*: X_{\omega} \rightarrow Y_{\omega}$ obedece ao axioma (Mon2), ou seja, se A for um subconjunto infinito de $X$, então $A \subsetneq{ }^{*} A$.

Demonstração. Temos $\omega_{1} \leqslant|A|^{+}$e o ultrafiltro $\mathcal{U}$ é $|A|^{+}$-incompleto, resultando que a imersão canônica $e: A \rightarrow \prod_{\mathcal{U}} A$ não é sobrejetora (Proposição 3.60). Seja $u: \prod_{\mathcal{U}} A \rightarrow^{*} A$ a bijeção canônica (Proposição 3.29). Portanto, a composição $u \circ e: A \rightarrow{ }^{*} A$ não é sobrejetora, e, como $(u \circ e)(x)={ }^{*} x(\forall x \in A)$, existe um elemento de ${ }^{*} A$ que não é da forma ${ }^{*} x$ para $x \in A$.

Com o intuito de demonstrar que $*$ obedece ao axioma (Mon3), apontaremos os seguintes lemas:

Lema 5.22. Seja $C(i, x)$ uma condição nas variáveis $i$ e $x$, e seja $r \in W_{\omega}$. As condições

$$
\left[\left(\exists x \in X_{\omega}\right)(x \in r(i) \wedge C(i, x))\right] \text { q.t.p. }
$$




$$
\left(\exists s \in W_{\omega}\right)[(s(i) \in r(i) \wedge C(i, s(i))) \text { q.t.p. }]
$$

são equivalentes.

Demonstração. A prova de que a segunda condição implica na primeira é imediata. Suponha que existe um conjunto $U$ no ultrafiltro $\mathcal{U}$ tal que $\left(\exists x \in X_{\omega}\right)(x \in r(i) \wedge C(i, x))$ para todo $i \in U$. Para cada $i \in U$, seja $s(i)$ um $X_{\omega}$-objeto pertencente a $r(i)$ tal que $C(i, s(i))$, e, para cada $i \in I-U$, seja $s(i):=\emptyset$. Assim, definimos uma função $s: I \rightarrow X_{\omega}$ tal que $s(i) \in r(i)$ q.t.p. e $C(i, s(i))$ q.t.p. Pela Proposição 5.14, temos $s \in W_{\omega}$, provando a condição necessária da equivalência desejada.

Lema 5.23. Para toda $L_{\epsilon}$-fórmula $\phi\left(x_{1} \ldots x_{n}\right)$ com quantificadores limitados, e para quaisquer $r_{1} \ldots r_{n} \in W_{\omega}$, temos

$$
\models \phi\left(r_{1}(i) \ldots r_{n}(i)\right) \text { q.t.p. } \Leftrightarrow * \models \phi\left(f\left(r_{1}\right) \ldots f\left(r_{n}\right)\right) \text {. }
$$

Demonstração. A prova é por indução sobre a complexidade de $\phi$. Suponha que $\phi\left(x_{1} \ldots x_{n}\right)$ é uma $L_{\epsilon}$-fórmula de complexidade $k$, e suponha que o resultado do Lema já foi provado para toda $L_{\epsilon}$-fórmula de complexidade $<k$. Sejam $r_{1} \ldots r_{n} \in W_{\omega}$.

- Se $\phi\left(x_{1} \ldots x_{n}\right)$ for uma $L_{\epsilon}$-fórmula atômica, então $n=2$, e $\phi=x_{1} \in x_{2}$ ou $\phi=x_{1}=x_{2}$. No primeiro caso, as seguintes condições serão equivalentes:
1. $\models \phi\left(r_{1}(i) \ldots r_{n}(i)\right)$ q.t.p.;
4. $f\left(r_{1}\right) \in f\left(r_{2}\right)$;
2. $\models r_{1}(i) \in r_{2}(i)$ q.t.p.;
5. * $=f\left(r_{1}\right) \in f\left(r_{2}\right)$;
3. $r_{1}(i) \in r_{2}(i)$ q.t.p.;
6. $* \models \phi\left(f\left(r_{1}\right) \ldots f\left(r_{n}\right)\right)$,

onde usamos a Proposição 5.16 na equivalência $3 \Leftrightarrow 4$. A prova do caso $\phi=x_{1}=x_{2}$ é análoga.

- Se $\phi\left(x_{1} \ldots x_{n}\right)$ for da forma

$$
\phi\left(x_{1} \ldots x_{n}\right)=\neg \chi\left(x_{1} \ldots x_{n}\right)
$$

então a $L_{\epsilon}$-fórmula $\chi$ terá complexidade $k-1$, e as seguintes condições serão equivalentes:
1. $\models \phi\left(r_{1}(i) \ldots r_{n}(i)\right)$ q.t.p.;
3. * $\forall \neq \chi\left(f\left(r_{1}\right) \ldots f\left(r_{n}\right)\right)$;
2. $\forall \chi \chi\left(r_{1}(i) \ldots r_{n}(i)\right)$ q.t.p.;
4. $* \models \phi\left(f\left(r_{1}\right) \ldots f\left(r_{n}\right)\right)$,

onde usamos a hipótese de indução em $2 \Leftrightarrow 3$. 
- Se $\phi\left(x_{1} \ldots x_{n}\right)$ for da forma

$$
\phi\left(x_{1} \ldots x_{n}\right)=\left(\chi\left(x_{1} \ldots x_{n}\right) \wedge \psi\left(x_{1} \ldots x_{n}\right)\right),
$$

então as $L_{\epsilon}$-fórmulas $\chi$ e $\psi$ terão complexidades menores que $k$, e as seguintes condições serão equivalentes:

1. $\models \phi\left(r_{1}(i) \ldots r_{n}(i)\right)$ q.t.p.;

2. $\models \chi\left(r_{1}(i) \ldots r_{n}(i)\right)$ q.t.p. e $\models \psi\left(r_{1}(i) \ldots r_{n}(i)\right)$ q.t.p.;

3. $* \models \chi\left(f\left(r_{1}\right) \ldots f\left(r_{n}\right)\right)$ e $* \models \psi\left(f\left(r_{1}\right) \ldots f\left(r_{n}\right)\right)$;

4. $* \models \phi\left(f\left(r_{1}\right) \ldots f\left(r_{n}\right)\right)$,

onde usamos a hipótese de indução em $2 \Leftrightarrow 3$.

- Se $\phi\left(x_{1} \ldots x_{n}\right)$ for da forma $(\exists y) \chi\left(x_{1} \ldots x_{n}, y\right)$ (onde $y$ é uma variável que não aparece na lista $x_{1} \ldots x_{n}$ ), então, como $\phi$ tem quantificadores limitados (Definição A.16), a $L_{\epsilon}$-fórmula $\chi\left(x_{1} \ldots x_{n}, y\right)$ será da forma

$$
\chi\left(x_{1} \ldots x_{n}, y\right)=y \in x_{m} \wedge \psi\left(x_{1} \ldots x_{n}, y\right),
$$

onde $1 \leqslant m \leqslant n$ e $\psi\left(x_{1} \ldots x_{n}, y\right)$ é uma $L_{\epsilon}$-fórmula. Sem perda de generalidade assumiremos $m=1$. As condições

$$
\begin{gathered}
* \models \phi\left(f\left(r_{1}\right) \ldots f\left(r_{n}\right)\right) ; \\
\left(\exists g \in Y_{\omega}\right) * \models \chi\left(f\left(r_{1}\right) \ldots f\left(r_{n}\right), g\right) ; \\
\left(\exists g \in f\left(r_{1}\right) \cap Y_{\omega}\right) * \models \psi\left(f\left(r_{1}\right) \ldots f\left(r_{n}\right), g\right)
\end{gathered}
$$

são equivalentes. Como $Y$ é um conjunto base, se $g \in f\left(r_{1}\right) \cap Y_{\omega}$, então $f\left(r_{1}\right) \notin Y$, $r_{1} \notin W_{0}, \mathrm{e}$

$$
g \in f\left(r_{1}\right)=\left\{f(s) \vdots s \in W_{\omega} \text { e } s(i) \in r_{1}(i) \text { q.t.p. }\right\}
$$

pelo Corolário 5.15, implicando que existe um $s \in W_{\omega}$ tal que $s(i) \in r_{1}(i)$ q.t.p. e $g=f(s)$. Portanto, como a fórmula $\psi$ tem complexidade $k-2$, as seguintes condições são equivalentes:

1. $* \models \phi\left(f\left(r_{1}\right) \ldots f\left(r_{n}\right)\right)$;

2. $\left(\exists g \in f\left(r_{1}\right) \cap Y_{\omega}\right) * \models \psi\left(f\left(r_{1}\right) \ldots f\left(r_{n}\right), g\right)$;

3. $\left(\exists s \in W_{\omega} \operatorname{com} s(i) \in r(i)\right.$ q.t.p. $) * \models \psi\left(f\left(r_{1}\right) \ldots f\left(r_{n}\right), f(s)\right)$;

4. $\left(\exists s \in W_{\omega} \operatorname{com} s(i) \in r(i)\right.$ q.t.p. $) \models \psi\left(r_{1}(i) \ldots r_{n}(i), s(i)\right)$ q.t.p.;

5. $\left(\exists s \in W_{\omega}\right) \quad\left(\models s(i) \in r_{1}(i) \wedge \psi\left(r_{1}(i) \ldots r_{n}(i), s(i)\right)\right.$ q.t.p. $)$;

6. $\left(\left(\exists x \in X_{\omega}\right) \models x \in r_{1}(i) \wedge \psi\left(r_{1}(i) \ldots r_{n}(i), x\right)\right)$ q.t.p.;

7. $\models \phi\left(r_{1}(i) \ldots r_{n}(i)\right)$ q.t.p., 
onde usamos a hipótese de indução na equivalência $3 \Leftrightarrow 4$ e usamos o Lema 5.22 em $5 \Leftrightarrow 6$.

- As provas dos casos

$$
\phi\left(x_{1} \ldots x_{n}\right)=\chi\left(x_{1} \ldots x_{n}\right) \vee \psi\left(x_{1} \ldots x_{n}\right)
$$

$\mathrm{e}$

$$
\phi\left(x_{1} \ldots x_{n}\right)=\left(\forall y \in x_{1}\right) \psi\left(x_{1} \ldots x_{n}, y\right)
$$

são consequências imediatas dos casos que provamos e das identidades lógicas

$$
\chi \vee \psi \Leftrightarrow[\neg((\neg \chi) \wedge(\neg \psi))]
$$

e

$$
\left(\forall y \in x_{1}\right) \psi \Leftrightarrow\left(\neg\left(\exists y \in x_{1}\right)(\neg \psi)\right)
$$

respectivamente.

A indução está completa, provando que o resultado desejado é válido para toda $L_{\epsilon}$-fórmula $\phi\left(x_{1} \ldots x_{n}\right)$ com quantificadores limitados.

Proposição 5.24. A função $*: X_{\omega} \rightarrow Y_{\omega}$ satisfaz ao axioma (Mon3).

Demonstração. Se $\phi\left(x_{1} \ldots x_{n}\right)$ for uma $L_{\epsilon}$-fórmula com quantificadores limitados, e se $a_{1} \ldots a_{n}$ forem $X_{\omega}$-objetos, então pelo Lema 5.23 temos a equivalência

$$
\models \phi\left(p\left(a_{1}\right)(i) \ldots p\left(a_{n}\right)(i)\right) \text { q.t.p. } \Leftrightarrow * \models \phi\left(f\left(p\left(a_{1}\right)\right) \ldots f\left(p\left(a_{n}\right)\right)\right) .
$$

Como $p\left(a_{m}\right)(i)=a_{m}$ para quaisquer $m \in[1, n]_{\mathbb{N}}$ e $i \in I$, e como $I \in \mathcal{U}$, temos

$$
\models \phi\left(a_{1} \ldots a_{n}\right) \Leftrightarrow * \models \phi\left({ }^{*} a_{1} \ldots{ }^{*} a_{n}\right) \text {. }
$$

Em nossa construção do conjunto base $Y$ e em nossa verificação de que a função * satisfaz aos axiomas (Mon1), (Mon2) e (Mon3), fizemos duas suposições cruciais em relação ao conjunto não nulo $I$ e ao ultrafiltro $\mathcal{U}$ sobre $I$ : as Suposições 5.5 e 5.19. No Exemplo 5.6 e na Observação 5.20 vimos que existem infinitas escolhas de $I$ e $\mathcal{U}$ tais que essas duas suposições sejam obedecidas, e a prova do principal teorema desta dissertação está completa.

Teorema 5.25 (Existência dos Monomorfismos Não Standard). Se $X$ for um conjunto base, então existirão um conjunto base $Y$ e um monomorfismo não standard $*: X_{\omega} \rightarrow Y_{\omega}$.

A concepção de * que elaboramos é dita ser uma construção por ultraprodutos. Existem outras construções dessa variedade que obtêm monomorfismos não standard com particularidades desejáveis, e também existem construções dessas funções que não envolvem ultraprodutos. 


\section{$5.6 * \mathbb{Q}$ e $* \mathbb{R}$ são Cauchy-completos}

Encerraremos este capítulo mostrando que, dependendo do monomorfismo não standard * considerado, é possível concluir que os corpos ordenados dos números hiperracionais e hiper-reais, ${ }^{*} \mathbb{Q}$ e $* \mathbb{R}$, são Cauchy-completos.

Assumiremos que $X$ contém uma cópia do conjunto dos números racionais, e escreveremos $\mathbb{Q} \subset X$.

Lema 5.26. Para quaisquer $A, B \in X^{\omega}$ e para toda função $h: *\langle A\rangle \rightarrow{ }^{*} B$, existe uma extensão interna $H:{ }^{*} A \rightarrow{ }^{*} B$ de $h$.

Demonstração. Para cada $a \in A$, temos $h\left({ }^{*} a\right) \in{ }^{*} B$ e existe uma função $t_{a}: I \rightarrow B$ tal que $h\left({ }^{*} a\right)=f\left(t_{a}\right)$ (Corolário 5.18). Seja $T: I \rightarrow{ }^{A} B$ a função dada por $T(i)(a):=t_{a}(i)$. Como ${ }^{A} B \in X^{\omega}$ (Teorema 4.8, Letra $(l)$ ), temos (Teorema 4.32, Letra $(h)$ )

$$
f(T) \in{ }^{*}\left({ }^{A} B\right)=\mathbb{I}^{\omega} \cap\left({ }^{*} A\right)\left({ }^{*} B\right),
$$

ou seja, $f(T)$ é uma função interna do tipo ${ }^{*} A \rightarrow{ }^{*} B$. Seja $H:=f(T)$. Pela definição da função $T$, para cada $a \in A$ a condição

$$
\models\left(p(a)(i), t_{a}(i)\right) \in T(i)
$$

é verdadeira para todo $i \in I$, e, pelo Lema 5.23, temos

$$
* \models\left(f(p(a)), f\left(t_{a}\right)\right) \in f(T),
$$

ou seja, $* \models\left({ }^{*} a, h\left({ }^{*} a\right)\right) \in H$, implicando em $H\left({ }^{*} a\right)=h\left({ }^{*} a\right)(\forall a \in A)$.

Lema 5.27. Seja $\mathcal{K}$ um $X_{\omega}$-conjunto tal que ${ }^{*} \mathcal{K} \subset{ }^{*} X^{\omega}$. Se $\mathcal{B}$ for um subconjunto enumerável de ${ }^{*} \mathcal{K}$ que satisfaz à PIF (Definição 3.10), então $\bigcap \mathcal{B} \neq \emptyset$.

Demonstração. Sejam $B_{1} B_{2} \ldots B_{n} \ldots$ os elementos de $\mathcal{B}$, e seja $h: \mathbb{N} \rightarrow{ }^{*} \mathcal{K}$ a função dada por $h(n):=B_{n}$. Existe uma extensão interna $H:{ }^{*} \mathbb{N} \rightarrow{ }^{*} \mathcal{K}$ de $h$ (Lema 5.26), e, como a união $U:=\bigcup^{*} \mathcal{K}$ é interna (Teorema 4.25, Letra $(b)$ ), o conjunto

$$
G:=\left\{n \in{ }^{*} \mathbb{N}: * \models(\exists x \in U)\left(\forall m \in{ }^{*} \mathbb{N}\right)(m \leqslant n \longrightarrow x \in H(m))\right\}
$$

é interno pelo PDI. Como $\mathcal{B}$ observa a PIF, temos $\mathbb{N} \subset G$ e existe um número hipernatural infinito $N$ pertencente a $G$ (Teorema 4.51 , Letra $(a)$ ), resultando que existe um $x \in U$ tal que

$$
x \in \bigcap_{m \in[1, N]_{*}} H(m) \subset \bigcap_{m \in \mathbb{N}} H(m)=\bigcap_{m \in \mathbb{N}} h(m)=\bigcap \mathcal{B} .
$$


A propriedade de $*$ descrita no enunciado do Lema 5.27 é chamada de $\omega_{1}$-saturação. Assim, o monomorfismo não standard $*$ descrito na Definição 5.17 é $\omega_{1}$-saturado.

Teorema 5.28. Toda sequência de Cauchy no corpo ordenado dos números hiper-racionais é eventualmente constante.

Demonstração. Suponha que $x_{1} x_{2} x_{3} \ldots$ é uma sequência de Cauchy em ${ }^{*} \mathbb{Q}$ que não é eventualmente constante. Para cada $n \in \mathbb{N}$, seja $m_{n}$ o menor índice maior que $n$ tal que $x_{n} \neq x_{m_{n}}$, e seja $y_{n}:=\left|x_{n}-x_{m_{n}}\right|$. Seja $\mathcal{B}$ o conjunto de intervalos em $* \mathbb{Q}$ dado por $\left\{\left(0, y_{n}\right)_{* \mathbb{Q}}\right\}_{n \geqslant 1}$. Como todo elemento de $\mathcal{B}$ é interno (Exemplo 2.28), temos (Teorema 4.32, Letra $(g))$

$$
\mathcal{B} \subset \mathbb{I}^{\omega} \cap \mathcal{P}\left({ }^{*} \mathbb{Q}\right)={ }^{*}(\mathcal{P}(\mathbb{Q}))
$$

e, como $\mathcal{B}$ é enumerável e satisfaz à PIF, existirá um número hiper-racional positivo $r$ tal que $r<y_{n}(\forall n)$ (Lema 5.27). Assim, como supomos que $\left\{x_{n}\right\}$ é uma sequência de Cauchy, existe um número natural $N$ tal que para quaisquer números naturais $m$ e $n$ temos a implicação

$$
m, n \geqslant N \Rightarrow\left|x_{m}-x_{n}\right|<r,
$$

o que é absurdo, visto que $N, m_{N} \geqslant N$ e $r<y_{N}=\left|x_{N}-x_{m_{N}}\right|$. Portanto, provamos que toda sequência de Cauchy em $* \mathbb{Q}$ é eventualmente constante.

Trocando toda ocorrência do conjunto $\mathbb{Q}$ por $\mathbb{R}$ nesta seção, obtém-se uma demonstração completa de que toda sequência de Cauchy em * $\mathbb{R}$ é eventualmente constante. Assim, ${ }^{*} \mathbb{R}$ é Cauchy-completo. 
Apêndice 



\section{A \\ Conceitos da Teoria dos Modelos}

Diversos conceitos da Teoria dos Modelos, área que estuda classes de estruturas matemáticas sob a perspectiva da Lógica Matemática, são imprescindíveis para o texto principal desta dissertação. Este apêndice tem o propósito de recapitular esses conceitos, assim como prestar esclarecimentos acerca das definições e terminologias relevantes que serão amplamente empregadas ao longo do trabalho. Para uma exposição detalhada, ver $(13,28,39)$.

\section{A.1 Monóides Livres}

A codificação de mensagens na maioria dos sistemas de escrita concebidos na história da humanidade consiste em sequências horizontais de símbolos engravados em uma tela, e esses símbolos devem pertencer a um conjunto, o alfabeto do sistema. O correspondente a esse procedimento na Teoria dos Conjuntos (ZFC ou NBG) é desempenhado através dos monóides livres.

Seja $\mathbb{A}$ um conjunto. Considere a função binária

$$
.: \operatorname{SeqF}(\mathbb{A}) \times \operatorname{SeqF}(\mathbb{A}) \rightarrow \operatorname{SeqF}(\mathbb{A})
$$

definida por

$$
\begin{aligned}
\left(a_{1} a_{2} \ldots a_{m}\right) \cdot\left(b_{1} b_{2} \ldots b_{n}\right): & =a_{1} a_{2} \ldots a_{m} b_{1} b_{2} \ldots b_{n} \\
& =\left\{\begin{array}{ll}
a_{i} & \text { se } i \leqslant m \\
b_{i-m} & \text { se } m<i
\end{array}\right\}_{1 \leqslant i \leqslant m+n} .
\end{aligned}
$$

Note que

$$
\begin{aligned}
\left(\left(a_{1} \ldots a_{m}\right) \cdot\left(b_{1} \ldots b_{n}\right)\right) \cdot\left(c_{1} \ldots c_{p}\right) & =\left(a_{1} \ldots a_{m}\right) \cdot\left(\left(b_{1} \ldots b_{n}\right) \cdot\left(c_{1} \ldots c_{p}\right)\right) \\
& =a_{1} \ldots a_{m} b_{1} \ldots b_{n} c_{1} \ldots c_{p}
\end{aligned}
$$

e

$$
\emptyset .\left(a_{1} a_{2} \ldots a_{m}\right)=\left(a_{1} a_{2} \ldots a_{m}\right) . \emptyset=a_{1} a_{2} \ldots a_{m}
$$

para quaisquer sequências finitas $\left\{a_{i}\right\}_{i \leqslant m},\left\{b_{i}\right\}_{i \leqslant n},\left\{c_{i}\right\}_{i \leqslant p} \in \operatorname{SeqF}(\mathbb{A})$, de modo que a função binária que definimos é uma lei de composição associativa em SeqF $(\mathbb{A})$ que tem $\emptyset$ como elemento neutro. Assim, o conjunto $\operatorname{SeqF}(\mathbb{A})$ é um monóide quando munido dessa lei, o qual é chamado de monóide livre sobre $\mathbb{A}$. A lei de composição em $\operatorname{SeqF}(\mathbb{A})$ será denotada por justaposição, ou seja, a composição de quaisquer $A$ e $B$ em $\operatorname{SeqF}(\mathbb{A})$ será 
denotada por $A B$. Os elementos de $\operatorname{SeqF}(\mathbb{A})$ são chamados de palavras em $\operatorname{SeqF}(\mathbb{A})$, e todo subconjunto de $\operatorname{SeqF}(\mathbb{A})$ é dito ser uma linguagem com alfabeto $\mathbb{A}$.

Observação A.1. Na Teoria dos Monóides, é comum estabelecer que um produtório nulo é igual ao elemento neutro do monóide. Assim, temos que um produtório da forma $A_{1} \ldots A_{n}$ $\operatorname{com} A_{i} \in \operatorname{SeqF}(\mathbb{A})(\forall i)$ será igual a $\emptyset$ no caso $n=0$.

Definição A.2. Sejam $A$ e $B$ duas palavras em $\operatorname{SeqF}(\mathbb{A})$. Dizemos que $A$ é uma subpalavra de $B$ se existirem palavras $C$ e $D$ em $\operatorname{SeqF}(\mathbb{A})$ tais que $B=C A D$.

\section{A.2 Linguagens; Substituição Simultânea}

Em certas situações, é necessário e relevante que alguns símbolos em uma palavra sejam substituídos por outras palavras.

Definição A.3. Se $S \subset \mathbb{N}$, então uma função $s: S \rightarrow \operatorname{SeqF}(\mathbb{A})$ é dita ser um esquema de substituição (em $\operatorname{SeqF}(\mathbb{A}))$.

Definição A.4. Seja $\mathbb{A}$ um alfabeto, seja $A=a_{1} a_{2} \ldots a_{k}\left(\operatorname{com} a_{i} \in \mathbb{A}(\forall i)\right)$ uma palavra em $\operatorname{SeqF}(\mathbb{A})$, e seja

$$
s:\left\{i_{1}<i_{2}<\ldots<i_{n}\right\} \rightarrow \operatorname{SeqF}(\mathbb{A})
$$

um esquema de substituição (com $i_{n} \leqslant k$ ). A palavra $A\ulcorner s\urcorner$ obtida de $A$ por substituição simultânea das letras de posições $i_{1} \ldots i_{n}$ por $s\left(i_{1}\right) \ldots s\left(i_{n}\right)$, respectivamente, é definida por

$$
\begin{array}{r}
A\ulcorner s\urcorner:=a_{1} \ldots a_{i_{1}-1} s\left(a_{i_{1}}\right) a_{i_{1}+1} \ldots a_{i_{2}-1} s\left(a_{i_{2}}\right) a_{i_{2}+1} \ldots \\
\ldots a_{i_{n}-1} s\left(a_{i_{n}}\right) a_{i_{n}+1} \ldots a_{k} .
\end{array}
$$

\section{A.3 Assinaturas}

Os símbolos matemáticos não lógicos têm papéis sintáticos específicos nos termos e sentenças nas quais eles ocorrem. É conveniente que cada um desses símbolos seja enquadrado em uma categoria correspondente a exatamente uma dessas aplicações sintáticas.

Definição A.5. Uma assinatura é uma quadra ordenada

$$
L:=\left(\mathbb{S}_{\text {rel }}^{L}, \mathbb{S}_{\text {fun }}^{L}, \mathbb{S}_{c o n}^{L}, \operatorname{ar}^{L}\right)
$$

onde:

- $\mathbb{S}_{\text {rel }}^{L}, \mathbb{S}_{\text {fun }}^{L}$ e $\mathbb{S}_{c o n}^{L}$ são conjuntos disjuntos possivelmente nulos;

- $\operatorname{ar}^{L}$ é uma função do tipo $\mathbb{S}_{\text {rel }}^{L} \cup \mathbb{S}_{\text {fun }}^{L} \cup\{=\} \rightarrow \mathbb{N} \operatorname{com} \operatorname{ar}^{L}(=)=2$. 
Nesse caso, dizemos que:

- Os elementos de $\mathbb{S}_{\text {rel }}^{L}$ são os símbolos relacionais em $L$, os quais denotaremos pela letra $P$;

- Para cada número natural $n$, os elementos $P$ de $\mathbb{S}_{\text {rel }}^{L}$ tais que $\operatorname{ar}^{L}(P)=n$ são os símbolos relacionais $n$-ários em $L$;

- Os elementos de $\mathbb{S}_{f u n}^{L}$ são os símbolos funcionais em $L$, os quais denotaremos pela letra $G$;

- Para cada número natural $n$, os elementos $G$ de $\mathbb{S}_{f u n}^{L}$ tais que $\operatorname{ar}^{L}(G)=n$ são os símbolos funcionais $n$-ários em $L$;

- Os elementos de $\mathbb{S}_{c o n}^{L}$ são os símbolos de constante em $L$, os quais denotaremos pela letra $c$;

- Para cada $S \in \mathbb{S}_{\text {fun }}^{L} \cup \mathbb{S}_{\text {rel }}^{L} \cup\{=\}$, o número natural $\operatorname{ar}^{L}(S)$ é dito ser a aridade de $S$ em $L$.

Em geral, identificamos $L$ com a união disjunta $\mathbb{S}_{\text {rel }}^{L} \cup \mathbb{S}_{\text {fun }}^{L} \cup \mathbb{S}_{c o n}^{L}$, e dizemos que os elementos dessa união são elementos de $L$. Em particular, quando os conjuntos $\mathbb{S}_{\text {rel }}^{L}$, $\mathbb{S}_{\text {fun }}^{L}$ e $\mathbb{S}_{c o n}^{L}$ forem finitos, denotaremos a assinatura $L$ utilizando-se da notação usual $\left\{x_{1} x_{2} \ldots x_{n}\right\}$ para conjuntos finitos, onde a situação de cada símbolo da lista como um símbolo relacional $n$-ário, um símbolo funcional $n$-ário ou um símbolo de constante deverá ser explicitada pelo o contexto.

Exemplo A.6. No caso em que

$$
\mathbb{S}_{\text {rel }}^{L}=\mathbb{S}_{\text {fun }}^{L}=\mathbb{S}_{\text {con }}^{L}=\emptyset
$$

dizemos que $L$ é a assinatura vazia, ou $L=\emptyset$ em símbolos.

Exemplo A.7. A assinatura $L_{\epsilon}:=\{\in\}$ tal que $\in$ é um símbolo relacional binário é dita ser a assinatura dos conjuntos.

Exemplo A.8. A assinatura $L_{G}:=\left\{\cdot{ }^{-1}, e\right\}$ tal que

- · é um símbolo funcional binário;

- e é um símbolo de constante

- ${ }^{-1}$ é um símbolo funcional unário;

é dita ser a assinatura dos grupos. 
Exemplo A.9. A assinatura $L_{A}:=\{+, \cdot, 0,1\}$ tal que

- + e · são símbolos funcionais binários;

- 0 e 1 são símbolos de constante

é dita ser a assinatura dos anéis.

Exemplo A.10. A assinatura $L_{A O}:=\{+, \cdot, 0,1,<\}$ tal que

- + e $\cdot$ são símbolos funcionais binários; • < é um símbolo relacional binário

- 0 e 1 são símbolos de constante;

é dita ser a assinatura dos anéis ordenados.

Definição A.11. Sejam $L$ e $L^{\prime}$ duas assinaturas. Dizemos que $L$ é uma subassinatura de $L^{\prime}$, ou $L^{\prime}$ é uma extensão de $L$, ou $L \subset L^{\prime}$ simbolicamente, se:

- $\mathbb{S}_{r e l}^{L} \subset \mathbb{S}_{r e l}^{L^{\prime}}$

- $\mathbb{S}_{\text {fun }}^{L} \subset \mathbb{S}_{\text {fun }}^{L^{\prime}}$
- $\mathbb{S}_{\text {con }}^{L} \subset \mathbb{S}_{\text {con }}^{L^{\prime}}$

- $\operatorname{ar}^{L}$ é uma restrição de $\operatorname{ar}^{L^{\prime}}$.

No restante deste apêndice, admitiremos que $L$ é uma assinatura.

As assinaturas contêm apenas os símbolos não lógicos de uma linguagem matemática. Um alfabeto matemático completo também deve conter os símbolos lógicos.

Definição A.12. Seja $L$ uma assinatura, e seja

$$
\mathbb{A}_{L}:=\left\{v_{1} v_{2} \ldots v_{i} \ldots\right\} \cup\{\neg, \vee, \wedge, \exists, \forall,=,),(,,\} \cup L,
$$

onde:

- A união tripla que define $\mathbb{A}_{L}$ é disjunta;

- $\left\{v_{1} v_{2} \ldots v_{i} \ldots\right\}$ é um conjunto enumerável de objetos $v_{i}$ distintos;

- Os símbolos

$$
\neg, \vee, \wedge, \exists, \forall,=,),(\text {, e , }
$$

são distintos.

O conjunto $\mathbb{A}_{L}$ é dito ser o alfabeto de assinatura $L$ com igualdade. Dizemos que: 
- $v_{1} v_{2} \ldots v_{i} \ldots$ são as variáveis;

- $\neg, \vee, \wedge, \exists, \forall$ são os símbolos lógicos em $\mathbb{A}_{L}$;

- Os elementos de $L$ são os símbolos específicos em $\mathbb{A}_{L}$;

- ᄀ é o símbolo da negação;

- V é o símbolo da disjunção;

- $\wedge$ é o símbolo da conjunção;
- $\exists$ é o símbolo da quantificação existencial;

- $\forall$ é o símbolo da quantificação universal;

- = é o símbolo da igualdade,$^{1}$ o qual é considerado um símbolo relacional binário em $L$ embora não seja um elemento de $L$;

- ( e ) são os parênteses; ${ }^{2}$

- , é a vírgula. ${ }^{3}$

As variáveis $v_{1} v_{2} v_{3} \ldots$ podem ser denotadas por qualquer outro símbolo, geralmente as letras $x, y$ e $z$ com ou sem subíndices. Assim, se $x_{1} \ldots x_{n}$ forem variáveis (distintas), então existirá uma sequência $i_{1} \ldots i_{n}$ de números naturais (distintos) tal que $x_{k}=v_{i_{k}}(\forall k)$.

\section{A.4 Termos}

Objetos matemáticos são representados pelas palavras em $\operatorname{SeqF}\left(\mathbb{A}_{L}\right)$ que são geradas por três regras sintáticas.

Definição A.13. Dizemos que uma palavra $t \in \operatorname{SeqF}\left(\mathbb{A}_{L}\right)$ é um $L$-termo ou um termo em $L$ se existe uma sequência finita não nula de palavras $t_{1} t_{2} \ldots t_{k}$ tal que $t_{k}=t$, e, para cada índice $i$, uma das seguintes condições é observada:

(Ter1) $t_{i}$ é uma variável;

(Ter2) $t_{i}$ é um símbolo de constante em $L$;

(Ter3) Existe um símbolo funcional $n$-ário $G$ em $L$ e existem índices $i_{1} i_{2} \ldots i_{n}<i$ tais que ${ }^{4}$

$$
t_{i}=G\left(t_{i_{1}}, t_{i_{2}}, \ldots, t_{i_{n}}\right)
$$

1 Note que o símbolo $=\in \mathbb{A}_{L}$ é (sutilmente) distinto do símbolo de igualdade $=$ da metateoria. Tal distinção foi introduzida para evitar certas ambiguidades possíveis, como por exemplo na condição $\phi=v_{1}=v_{2}$, a qual afirma que a palavra $\phi$ é formada pelos símbolos $v_{1},=$ e $v_{2}$, nesta ordem.

2 Não é necessário diferenciar os símbolos dos parênteses em $\mathbb{A}_{L}$ dos símbolos dos parênteses da metateoria (NBG), pois não há exemplos de possíveis ambiguidades resultantes dessa incúria.

3 O símbolo,$\in \mathbb{A}_{L}$ é (sutilmente) distinto da vírgula comum.

4 As virgulas do tipo, serão omitidas no restante do texto. 
A complexidade de um $L$-termo $t$ é o número de ocorrências de símbolos funcionais em $t$. O conjunto dos $L$-termos é uma linguagem com alfabeto $\mathbb{A}_{L}$ a qual é denotada por $\mathcal{T}(L)$. Denotaremos por $V(t)$ o conjunto dos índices das variáveis que ocorrem em um termo $t$, e dizemos que $t$ é um termo fechado se $V(t)=\emptyset$. A notação $t\left(v_{i_{1}} \ldots v_{i_{n}}\right)$ significará $V(t) \subset\left\{i_{1} \ldots i_{n}\right\}$, onde $i_{1} i_{2} \ldots i_{n} \in \mathbb{N}$, e, sempre que escrevermos $t\left(x_{1} \ldots x_{n}\right)$, estará subentendido que $x_{1} \ldots x_{n}$ são variáveis.

Um subtermo de uma palavra é uma subpalavra dessa palavra que é um termo.

\section{A.5 Fórmulas}

Algumas palavras em $\operatorname{SeqF}\left(\mathbb{A}_{L}\right)$ representam afirmações matemáticas completas.

Definição A.14. Dizemos que uma palavra $\phi \in \operatorname{SeqF}\left(\mathbb{A}_{L}\right)$ é uma $L$-fórmula ou uma fórmula em $L$ (de primeira ordem) se existe uma sequência finita não nula de palavras $\phi_{1} \phi_{2} \ldots \phi_{k}$ tal que $\phi_{k}=\phi$, e, para todo índice $i$, uma das seguintes condições é observada:

(For1) Existem um símbolo relacional $n$-ário $P$ em $L$ e $L$-termos $t_{1} t_{2} \ldots t_{n}$ tais que

$$
\phi_{i}=P\left(t_{1} t_{2} \ldots t_{n}\right)
$$

Tais fórmulas são chamadas de fórmulas atômicas;

(For2) Existe um índice $j<i$ tal que $\phi_{i}=\left(\neg \phi_{j}\right)$;

(For3) Existem índices $j, k<i$ tais que $\phi_{i}=\left(\phi_{j} \vee \phi_{k}\right)$;

(For4) Existem índices $j, k<i$ tais que $\phi_{i}=\left(\phi_{j} \wedge \phi_{k}\right)$;

(For5) Existem um índice $j<i$ e uma variável $x$ tais que $\phi_{i}=(\exists x) \phi_{j}$;

(For6) Existem um índice $j<i$ e uma variável $x$ tais que $\phi_{i}=(\forall x) \phi_{j}$.

Nota. Em ocasiões, omitiremos pares de parênteses que não forem estritamente necessários para evitar ambiguidades em algumas fórmulas. Porém, em outras ocasiões, adicionaremos pares de parênteses que ajudem na "leitura semântica" das fórmulas.

A complexidade de uma $L$-fórmula $\phi$ é o número de ocorrências dos símbolos $\neg, \vee, \wedge$, $\exists$ e $\forall$ em $\phi$. O conjunto das $L$-sentenças é uma linguagem com alfabeto $\mathbb{A}_{L}$, a qual é chamada de linguagem de primeira ordem de assinatura $L$ com igualdade e é denotada por $L_{\omega \omega}^{=}$.

Notação A.15. Para quaisquer $L$-fórmulas $\phi_{1}$ e $\phi_{2}$, as $L$-fórmulas

$$
\left(\left(\neg \phi_{1}\right) \vee \phi_{2}\right) \text { e }\left(\left(\left(\neg \phi_{1}\right) \vee \phi_{2}\right) \wedge\left(\left(\neg \phi_{2}\right) \vee \phi_{1}\right)\right)
$$


são respectivamente denotadas por

$$
\left(\phi_{1} \longrightarrow \phi_{2}\right) \text { e }\left(\phi_{1} \longleftrightarrow \phi_{2}\right)
$$

Fórmulas da forma $\left(\phi_{1} \longrightarrow \phi_{2}\right)$ são implicações, e fórmulas da forma $\left(\phi_{1} \longleftrightarrow \phi_{2}\right)$ são duplas implicações ou equivalências.

Uma subfórmula de uma palavra é uma subpalavra dessa palavra que é uma fórmula.

\section{A.6 Fórmulas em $L_{\epsilon}$ com Quantificadores Limitados}

As fórmulas mais relevantes para o estudo da Análise Não Standard são fórmulas em $L_{\epsilon}$ (Exemplo A.7) que podem conter quantificadores apenas em certas configurações específicas.

Definição A.16. Dizemos que uma $L_{\epsilon}$-fórmula $\phi$ tem quantificadores limitados se as seguintes condições são observadas:

(QLim1) Para toda subfórmula de $\phi$ da forma $(\exists x) \chi$ para alguma variável $x$ e alguma fórmula $\chi$, a fórmula $\chi$ é da forma

$$
\chi=(x \in y \wedge \psi)
$$

para alguma variável $y$ e alguma fórmula $\psi$;

(QLim2) Para toda subfórmula de $\phi$ da forma $(\forall x) \chi$ para alguma variável $x$ e alguma fórmula $\chi$, a fórmula $\chi$ é da forma

$$
\chi=(x \in y \longrightarrow \psi)
$$

para alguma variável $y$ e alguma fórmula $\psi$.

Notação A.17. Para quaisquer variáveis $x$ e $y$ e para qualquer $L_{\epsilon}$-fórmula $\psi$, as $L_{\epsilon}$-fórmulas

$$
(\exists x)(x \in y \wedge \psi) \text { e }(\forall x)(x \in y \longrightarrow \psi)
$$

são respectivamente denotadas por

$$
(\exists x \in y) \psi \mathrm{e}(\forall x \in y) \psi
$$

Tais fórmulas têm quantificadores limitados.

Exemplo A.18. A $L_{\epsilon}$-fórmula

$$
\phi=(\forall x)(x \in y \vee y \neq z) \wedge z=w
$$


não tem quantificadores limitados, pois a fórmula

$$
(\forall x)(x \in y \vee y \neq z)
$$

é uma subfórmula de $\phi$, e a fórmula

$$
(x \in y \vee y \neq z)
$$

não é da forma $\left(x \in v_{i} \longrightarrow \psi\right)$. Assim, $\phi$ não satisfaz à condição (QLim2).

Exemplo A.19. A $L_{\epsilon}$-fórmula

$$
\phi=(\exists y)(y \in x \wedge y \notin z) \longrightarrow(\forall y)(y \notin z \vee y=w)
$$

tem quantificadores limitados, pois:

- A única subfórmula de $\phi$ da forma $\left(\exists v_{i}\right) \chi$ é a fórmula

$$
(\exists y)(y \in x \wedge y \notin z)
$$

e a fórmula $(y \in x \wedge y \notin z)$ é da forma $\left(y \in v_{i} \wedge \psi\right)$ para alguma variável $v_{i}$ (neste caso $x$ ) e para alguma fórmula $\psi$ (neste caso $y \notin z$ ), observando a condição (QLim1).

- A única subfórmula de $\phi$ da forma $\left(\forall v_{i}\right) \chi$ é a fórmula

$$
(\forall y)(y \notin z \vee y=w)
$$

e a fórmula $(y \notin z \vee y=w)$ é da forma $\left(y \in v_{i} \longrightarrow \psi\right)$ para alguma variável $v_{i}$ (neste caso $z$ ) e para alguma fórmula $\psi$ (neste caso $y=w$ ), observando a condição (QLim2).

\section{A.7 Variáveis Livres e Variáveis Ligadas}

É proveitoso classificar as ocorrências das variáveis que ocorrem em uma $L$-fórmula de acordo com o papel que ela desempenha na fórmula: denotar um objeto matemático fixo em um dado contexto ou representar a atuação de um quantificador.

Definição A.20. Seja $\phi=\phi_{1} \phi_{2} \ldots \phi_{n} \quad\left(\operatorname{com} \phi_{i} \in \mathbb{A}_{L}(\forall i)\right)$ uma $L$-fórmula, seja $t=t_{1} t_{2} \ldots t_{r}\left(\operatorname{com} t_{i} \in \mathbb{A}_{L}(\forall i)\right)$ um $L$-termo, e seja $x$ uma variável. Temos as definições:

- Dizemos que $x$ ocorre livre em $\phi$ na posição $i$ se $\phi_{i}=x$ e não existe uma subfórmula $\phi_{j} \phi_{j+1} \ldots \phi_{j+m}$ de $\phi$ das formas $(\exists x) \chi$ ou $(\forall x) \chi$ tal que $j \leqslant i \leqslant j+m$;

- Dizemos que $x$ ocorre livre em $\phi$ se $x$ ocorre livre em $\phi$ em alguma posição $i$. O conjunto dos índices das variáveis que ocorrem livres em $\phi$ é denotado por $V(\phi)$; 
- $\phi$ é dita ser uma $L$-sentença ou uma sentença em $L$ se $V(\phi)=\emptyset$;

- Dizemos que $x$ ocorre ligada em $\phi$ na posição $i$ se $x$ não ocorre livre em $\phi$ na posição $i$;

- Dizemos que $x$ ocorre ligada em $\phi$ se $x$ ocorre ligada em $\phi$ em alguma posição;

- Dizemos que $t$ é livre para $x$ em $\phi$ na posição $i$ se $x$ ocorre livre em $\phi$ na posição $i$, e, para cada índice $j$ tal que $t_{j}$ é uma variável, temos que $t_{j}$ ocorre livre na fórmula

$$
\phi_{1} \ldots \phi_{i-1} t \phi_{i+1} \ldots \phi_{n}
$$

na posição $i+j-1$;

- Dizemos que $t$ é livre para $x$ em $\phi$ se, para cada índice $i$ tal que $x$ ocorre livre em $\phi$ na posição $i$, temos que $t$ é livre para $x$ em $\phi$ na posição $i$.

A notação $\phi\left(v_{i_{1}} \ldots v_{i_{n}}\right)$ significará $V(\phi) \subset\left\{i_{1} \ldots i_{n}\right\}$, onde $i_{1} i_{2} \ldots i_{n} \in \mathbb{N}$, e, sempre que escrevermos $\phi\left(x_{1} \ldots x_{n}\right)$, estará subentendido que $x_{1} \ldots x_{n}$ são variáveis.

As propriedades básicas da função $\phi \mapsto V(\phi)$ estão retratadas no teorema a seguir:

Teorema A.21. Seja $P$ um simbolo relacional n-ário em $L$, sejam $t_{1} \ldots t_{n} L$-termos, sejam $\phi$ e $\chi$ duas L-fórmulas, e seja vi uma variável. Temos:
(a) $V\left(P\left(t_{1} \ldots t_{n}\right)\right)=\bigcup_{k=1}^{n} V\left(t_{k}\right)$;
(d) $V(\phi \wedge \chi)=V(\phi) \cup V(\chi)$;
(b) $V((\neg \phi))=V(\phi)$;
(e) $V\left(\left(\exists v_{i}\right) \phi\right)=V(\phi)-\{i\}$;
(c) $V(\phi \vee \chi)=V(\phi) \cup V(\chi)$;
(f) $V\left(\left(\forall v_{i}\right) \phi\right)=V(\phi)-\{i\}$.

\section{A.8 Substituição de Variáveis em Termos e Fórmulas}

O modo mais relevante do processo de substituição (Definição A.4) consiste na substituição de variáveis em termos e fórmulas por termos. Quando essa operação é executada sobre uma fórmula, evita-se alterar as variáveis ligadas e introduzir termos que contenham variáveis que possam estar ligadas a algum quantificador após a permuta.

\section{Definição A.22.}

(a) Seja

$$
t=t_{1} t_{2} \ldots t_{k}=t\left(x_{1} \ldots x_{n}\right)
$$

$\left(\right.$ com $\left.t_{i} \in \mathbb{A}_{L}(\forall i)\right)$ um $L$-termo, sejam $h_{1} \ldots h_{n} L$-termos, sejam $i_{1} i_{2} \ldots i_{p} \leqslant k$ os índices tais que cada $t_{i_{j}}$ é uma variável, e seja $s:\left\{i_{1} i_{2} \ldots i_{p}\right\} \rightarrow \mathcal{T}(L)$ o esquema 
de substituição definido por $s\left(i_{j}\right):=h_{u}$ quando $t_{i_{j}}=x_{u}$. A palavra $t\ulcorner s\urcorner$ é um $L$-termo, o qual é denotado por $t\left(h_{1} \ldots h_{n}\right)$.

(b) Seja

$$
\phi=\phi_{1} \phi_{2} \ldots \phi_{k}=\phi\left(x_{1} \ldots x_{n}\right)
$$

$\left(\operatorname{com} \phi_{i} \in \mathbb{A}_{L}(\forall i)\right)$ uma $L$-fórmula, sejam $h_{1} \ldots h_{n} L$-termos tais que cada $h_{u}$ é livre para $x_{u}$ em $\phi$, sejam $i_{1} i_{2} \ldots i_{p} \leqslant k$ os índices tais que cada $\phi_{i_{j}}$ é uma variável que ocorre livre em $\phi$ na posição $i_{j}$, e seja $s:\left\{i_{1} i_{2} \ldots i_{p}\right\} \rightarrow \mathcal{T}(L)$ o esquema de substituição definido por $s\left(i_{j}\right):=h_{u}$ quando $\phi_{i_{j}}=x_{u}$. A palavra $\phi\ulcorner s\urcorner$ é uma $L$-fórmula, a qual é denotada por $\phi\left(h_{1} \ldots h_{n}\right)$.

\section{A.9 Estruturas}

Muitas estruturas matemáticas consistem em um conjunto munido de relações, funções e constantes definidas nesse conjunto. À vista disso, pode-se compreender uma assinatura (Definição A.5) como um esquema de construção adequado a um determinado tipo de estrutura, o qual contém as variedades de munições que devem ser fornecidas a um conjunto para que ele seja uma estrutura do tipo desejado.

Definição A.23. Uma $L$-estrutura $M$ é uma terna ordenada

$$
\left(\|M\|, L, \mathbb{I}^{M}\right)
$$

onde:

- $\|M\|$ é um conjunto não nulo, o qual é dito ser o universo de $M$. Na prática, por abuso de linguagem, é comum denotar o conjunto $\|M\|$ simplesmente por $M$ quando não houver risco de confusão;

- $\mathbb{I}^{M}$ é uma função com domínio $\mathbb{S}_{\text {rel }}^{L} \cup \mathbb{S}_{\text {fun }}^{L} \cup \mathbb{S}_{\text {con }}^{L} \cup\{=\}$ tal que:

- Para cada $P \in \mathbb{S}_{r e l}^{L}$, a imagem $\mathbb{I}^{M}(P)$ é uma relação $\operatorname{ar}^{L}(P)$-ária em $\|M\|$, a qual é dita ser a $M$-interpretação de $P$ e é denotada por $P^{M}$;

- Para cada $G \in \mathbb{S}_{\text {fun }}^{L}$, a imagem $\mathbb{I}^{M}(G)$ é uma função $\operatorname{ar}^{L}(G)$-ária em $\|M\|$, a qual é dita ser a $M$-interpretação de $G$, e ela é denotada por $G^{M}$;

- Para cada $c \in \mathbb{S}_{c o n}^{L}$, a imagem $\mathbb{I}^{M}(c)$ é um elemento de $M$, o qual é dito ser a $M$-interpretação de $c$ e é denotado por $c^{M}$;

- Definimos:

$$
\mathbb{I}^{M}(=)==^{M}:=\left\{(x, y) \in\|M\|^{2}: x=y\right\}
$$


Nesta dissertação, abriremos exceção para a regra $\|M\| \neq \emptyset$ somente quando a assinatura $L$ for tal que $\mathbb{S}_{\text {fun }}^{L}=\mathbb{S}_{\text {con }}^{L}=\emptyset$. Assim, por exemplo, consideraremos que a terna $\left(\emptyset, \emptyset, \mathbb{I}^{M}\right)$ é uma $\emptyset$-estrutura, onde $\mathbb{I}^{M}$ é a função definida em $\{=\}$ dada por $\mathbb{I}^{M}(=):=\emptyset$.

Exemplo A.24. Se $L$ for a assinatura vazia, então $L$ não terá símbolo algum para ser interpretado por uma $L$-estrutura $M$, implicando que tal estrutura sempre poderá ser identificada com seu conjunto de elementos. Ou seja, nesse caso podemos dizer que as L-estruturas são os conjuntos.

Definição A.25. Sejam $L^{\prime}$ uma assinatura que estende $L$, seja $M$ uma $L$-estrutura, e seja $N$ uma $L^{\prime}$-estrutura. Dizemos que $M$ é a redução de $N$ a uma $L$-estrutura, ou $N$ é uma expansão de $M$ a uma $L^{\prime}$-estrutura, se as seguintes condições são observadas:

- $\|M\|=\|N\|$;

- $P^{M}=P^{N}$ para cada símbolo relacional $P$ em $L$;

- $G^{M}=G^{N}$ para cada símbolo funcional $G$ em $L$;

- $c^{M}=c^{N}$ para cada símbolo de constante $c$ em $L$.

\section{A.10 Expansões Naturais}

Em algumas aplicações, é proveitoso estender o conjunto de símbolos de constante de uma assinatura.

Definição A.26. Seja $U$ um conjunto e seja $u \mapsto \underline{u}$ uma bijeção com domínio $U$ cuja imagem, $\underline{U}$, é disjunta de $L$. Denotaremos por $L(U)$ a extensão de $L$ definida por

- $\mathbb{S}_{r e l}^{L(U)}:=\mathbb{S}_{r e l}^{L}$

- $\mathbb{S}_{\text {fun }}^{L(U)}:=\mathbb{S}_{\text {fun }}^{L}$
- $\mathbb{S}_{c o n}^{L(U)}:=\mathbb{S}_{c o n}^{L} \cup \underline{U}$

- $\operatorname{ar}^{L(U)}:=\operatorname{ar}^{L}$.

Em palavras, a assinatura $L(U)$ é obtida de $L$ anexando a esta os símbolos de constante pertencentes ao conjunto $\underline{U}$.

Nota A.27. Podemos atribuir propriedades extras à bijeção $u \mapsto \underline{u}$ de acordo com a necessidade de cada situação. Exemplos dessas atribuições são:

- Se $U \cap L=\emptyset$, então poderemos assumir que $\underline{u}=u(\forall u \in U)$. Tal simplificação é bastante usada na literatura; 
- Sejam $\left\{u_{i}\right\}_{i \in I}$ e $\left\{u_{i}^{\prime}\right\}_{i \in I}$ duas famílias, e sejam $u_{i} \mapsto \underline{u_{i}}$ e $u_{i}^{\prime} \mapsto \underline{u_{i}^{\prime}}$ duas bijeções ${ }^{5}$ com respectivos domínios $\left\{u_{i}\right\}_{i \in I}$ e $\left\{u_{i}^{\prime}\right\}_{i \in I}$ cujas respectivas imagens, $\left\{\underline{u_{i}}\right\}_{i \in I}$ e $\left\{\underline{u_{i}^{\prime}}\right\}_{i \in I}$, são disjuntas de $L$. Podemos assumir que $\underline{u_{i}}=\underline{u_{i}^{\prime}}(\forall i \in I)$ quando tivermos a intenção de impor que os símbolos de constante anexados à assinatura $L$ na definição de $L\left(\left\{u_{i}\right\}_{i \in I}\right)$ sejam iguais aos símbolos de constante anexados à assinatura $L$ na definição de $L\left(\left\{u_{i}^{\prime}\right\}_{i \in I}\right)$. Nesse caso, os índices $i \in I$ operam como "etiquetas" que objetivam identificar cada símbolo de constante $\underline{u_{i}}=\underline{u_{i}^{\prime}}$ que será anexado a $L$.

Se $U$ for um subconjunto do universo de uma $L$-estrutura $M$, então uma maneira canônica de definir uma $L(U)$-estrutura consiste em interpretar os símbolos de constante adicionados a $L$ por eles próprios.

Definição A.28. Seja $M$ uma $L$-estrutura e seja $U \subset\|M\|$. Denotaremos por $M_{U}$ a $L(U)$-estrutura definida por

- $\left\|M_{U}\right\|=\|M\|$; - $c^{M_{U}}:=c^{M}\left(\forall c \in \mathbb{S}_{c o n}^{L}\right)$;

- $P^{M_{U}}:=P^{M}\left(\forall P \in \mathbb{S}_{r e l}^{L}\right)$;

- $G^{M_{U}}:=G^{M}\left(\forall G \in \mathbb{S}_{\text {fun }}^{L}\right)$; - $\underline{u}^{M_{U}}:=u(\forall u \in U)$.

A linguagem de NBG é gerada pela assinatura $L_{\epsilon}$, a qual contém apenas o símbolo relacional binário $\in$ representante da relação de pertinência, e todas as operações conjuntistas podem ser expressas por $L_{\epsilon}$-fórmulas. Tais sequências finitas de símbolos têm comprimentos enormes para a maioria dos teoremas relevantes de NBG, exigindo que notações sejam introduzidas na atividade matemática a fim de reduzir a aparência das fórmulas abordadas.

Quando apenas as fórmulas com quantificadores limitados (Definição A.16) são consideradas, para que esse processo de abreviação seja praticável é preciso que o universo no qual os objetos são considerados seja um conjunto $U$ predeterminado no contexto. Nesse caso, as noções matemáticas poderão ser descritas por $L_{\epsilon}(\mathrm{U})$-fórmulas, e, como o conjunto $U$ muitas vezes não é mencionado nas versões abreviadas dessas fórmulas, tais $L_{\epsilon}(\mathrm{U})$-fórmulas são tratadas como $L_{\epsilon}$-fórmulas por abuso de linguagem. As notações a seguir exemplificam essa prática, onde assumiremos que $\mathrm{U} \cap L=\emptyset$ e $\underline{u}=u(\forall u \in \mathrm{U})$.

Notação A.29. A $L_{\epsilon}(\mathrm{U})$-fórmula com quantificadores limitados dada por

$$
(\forall w \in \mathbf{U})(w \in z \longleftrightarrow(w=x \vee w=y))
$$

é denotada por $z=\{x, y\}$, para quaisquer variáveis $x, y, z$ e $w$. Se $t_{1}, t_{2}$ e $t_{3}$ forem $L_{\epsilon}(\mathrm{U})$-termos, então a substituição simultânea das respectivas variáveis $x, y$ e $z$ por $t_{1}$, $t_{2}$ e $t_{3}$ em $z=\{x, y\}$ será denotada por $t_{3}=\left\{t_{1}, t_{2}\right\}$.

5 Tais bijeções são comumente denotadas da mesma maneira, viz. com uma barra embaixo do objeto do domínio da função. Porém, em geral, elas são distintas. 
Notação A.30. A $L_{\epsilon}(\mathrm{U})$-fórmula com quantificadores limitados dada por

$$
(\forall w \in \mathrm{U})(w \in z \longleftrightarrow(w=\{x, x\} \vee w=\{x, y\}))
$$

é denotada por $z=(x, y)$, para quaisquer variáveis $x, y$, z e $w$. Se $t_{1}, t_{2}$ e $t_{3}$ forem $L_{\epsilon}(\mathrm{U})$-termos, então a substituição simultânea das respectivas variáveis $x, y$ e $z$ por $t_{1}$, $t_{2}$ e $t_{3}$ em $z=(x, y)$ será denotada por $t_{3}=\left(t_{1}, t_{2}\right)$.

Notações da forma $z=\ldots$ como as mostradas acima podem ser aproveitadas para definir notações de inúmeras outras formas, como mostra o exemplo a seguir.

Notação A.31. A $L_{\epsilon}(\mathrm{U})$-fórmula com quantificadores limitados dada por

$$
(\forall w \in \mathrm{U})(w=(x, y) \rightarrow w \in z)
$$

é denotada por $(x, y) \in z$ ou $y=z(x)$, onde assume-se (implicitamente) que uma variável distinta de $w$ está ligada ao quantificador presente na composição interna da fórmula $w=(x, y)$. Se $t_{1}, t_{2}$ e $t_{3}$ forem $L_{\epsilon}(\mathrm{U})$-termos, então a substituição simultânea das respectivas variáveis $x, y$ e $z$ por $t_{1}, t_{2}$ e $t_{3}$ em $(x, y) \in z$ será denotada por $\left(t_{1}, t_{2}\right) \in t_{3}$ ou $t_{2}=t_{3}\left(t_{1}\right)$.

\section{A.11 Interpretação de Termos Fechados}

Cada $L$-termo representa um objeto matemático, e, para cada $L$-estrutura $M$, há uma maneira de vincular cada $L$-termo a um elemento de $M$.

Definição A.32. Seja $M$ uma $L$-estrutura. Para cada $L$-termo fechado $t$ definiremos o elemento $t^{M}$ de $M$ indutivamente sobre a complexidade de $t$. Se $t$ tem complexidade 0, então $t$ é um símbolo de constante em $L$, e, nesse caso, definimos $t^{M}:=\mathbb{I}^{M}(t)$. Para um número natural $k$, suponha que $t^{M}$ está definido para $L$-termos fechados $t$ de complexidade $<k$, e suponha que $t$ é um $L$-termo fechado de complexidade $k$. O termo $t$ é da forma $G\left(w_{1} w_{2} \ldots w_{n}\right)$, onde $G$ é um símbolo funcional $n$-ário em $L$ e cada $w_{i}$ é um $L$-termo fechado. Assim, definimos:

$$
t^{M}:=G^{M}\left(w_{1}^{M} w_{2}^{M} \ldots w_{n}^{M}\right)
$$

Note que os termos $w_{1} w_{2} \ldots w_{n}$ têm complexidade $<k$, pois suas complexidades somam $k$ - 1. Para um $L$-termo fechado $t$, o elemento $t^{M}$ de $M$ é dito ser a $M$-interpretação de $t$.

Notação A.33. Seja $M$ uma $L$-estrutura. Se $t\left(x_{1} \ldots x_{n}\right)$ for um $L$-termo e $a_{1} \ldots a_{n} \in M$, então $t\left(\underline{a_{1}} \ldots \underline{a_{n}}\right)$ será um $L(M)$-termo fechado, e a sua $M_{M}$-interpretação, $t\left(\underline{a_{1}} \ldots \underline{a_{n}}\right)^{M_{M}}$, será denotada por

$$
t^{M}\left(a_{1} \ldots a_{n}\right)
$$




\section{A.12 Relação de Satisfatibilidade}

Cada $L$-fórmula é uma representação de uma afirmativa matemática, e, para cada $L$-estrutura $M$, há uma maneira formal de averiguar se uma $L$-fórmula simboliza uma asserção verdadeira ou falsa acerca da essência de $M$.

Definição A.34. Seja $M$ uma $L$-estrutura. Definiremos a relação de satisfatibilidade, $M \models \phi$, para toda $L(M)$-sentença $\phi$ indutivamente sobre a complexidade de $\phi$. Primeiramente, definimos $M \models \phi$ para $L(M)$-sentenças de complexidade 0 (ou seja, sentenças atômicas):

- Se $P$ for um símbolo relacional $n$-ário em $L$, e $t_{1} t_{2} \ldots t_{n}$ forem $L(M)$-termos fechados, então definiremos:

$$
M \models P\left(t_{1} t_{2} \ldots t_{n}\right): \Leftrightarrow\left(t_{1}^{M_{M}} t_{2}^{M_{M}} \ldots t_{n}^{M_{M}}\right) \in P^{M}
$$

Para um número natural $k$, suponha que a condição $M \models \phi$ está definida para $L(M)$-sentenças $\phi$ de complexidade $<k$, e suponha que $\phi$ é uma $L(M)$-sentença de complexidade $k$. Temos os 5 seguintes casos:

- Se $\phi$ for da forma $\left(\neg \phi^{\prime}\right)$, então definiremos:

$$
M \models \phi: \Leftrightarrow M \not \models \phi^{\prime} .
$$

Note que $\phi^{\prime}$ tem complexidade $k-1$;

- Se $\phi$ for da forma $\left(\phi_{1} \vee \phi_{2}\right)$, então definiremos:

$$
M \models \phi: \Leftrightarrow\left(M \models \phi_{1} \text { ou } M \models \phi_{2}\right) .
$$

Note que $\phi_{1}$ e $\phi_{2}$ têm complexidade $<k$, pois suas complexidades somam $k-1$;

- Se $\phi$ for da forma $\left(\phi_{1} \wedge \phi_{2}\right)$, então definiremos:

$$
M \models \phi: \Leftrightarrow\left(M \models \phi_{1} \text { e } M \models \phi_{2}\right) .
$$

Note que $\phi_{1}, \phi_{2}$ têm complexidade $<k$, pois suas complexidades somam $k-1$;

- Se $\phi$ for da forma $(\exists x) \chi(x)$, então definiremos:

$$
M \models \phi: \Leftrightarrow M \models \chi(\underline{a}) \quad(\exists a \in M) .
$$

Note que $\chi(\underline{a})$ tem complexidade $k-1$;

- Se $\phi$ for da forma $(\forall x) \chi(x)$, então definiremos:

$$
M \models \phi: \Leftrightarrow M \models \chi(\underline{a}) \quad(\forall a \in M) .
$$

Note que $\chi(\underline{a})$ tem complexidade $k-1$. 
Temos as seguintes terminologias:

- Para cada $L$-sentença $\phi$, dizemos que $M$ é um modelo de $\phi$ se a condição $M \models \phi$ é verdadeira;

- Para cada conjunto $\Phi$ de $L$-sentenças, dizemos que $M$ é um modelo de $\Phi$ se $M \models \phi(\forall \phi \in \Phi)$;

- Para quaisquer conjuntos $\Phi, \Psi$ e $\Omega$ de $L$-sentenças, dizemos que $\Phi$ semanticamente $^{6}$ implica $\Psi$ módulo $\Omega$, ou $\Phi \models_{\Omega} \Psi$ simbolicamente, se toda $L$-estrutura que é modelo de $\Phi \cup \Omega$ é modelo de $\Psi$. Quando $\Phi$ e $\Psi$ forem conjuntos unitários, digamos $\{\phi\}$ e $\{\psi\}$ respectivamente, a condição $\Phi \models_{\Omega} \Psi$ será denotada por $\phi \models_{\Omega} \psi$. Quando $\Omega=\emptyset$, tal conjunto pode ser omitido em todas essas notações;

- Para cada $L$-fórmula $\phi\left(x_{1} \ldots x_{n}\right)$ e para $a_{1} \ldots a_{n} \in M$, dizemos que $a_{1} \ldots a_{n}$ realiza $\phi$ em $M$, ou $M$ é um modelo de $\phi$ com parâmetros $a_{1} \ldots a_{n}$, se a condição $M \models \phi\left(\underline{a_{1}} \ldots \underline{a_{n}}\right)$ é verdadeira. Por vezes, denota-se tal condição por $M \models \phi\left[a_{1} \ldots a_{n}\right]$;

- Dizemos que uma $L$-sentença $\phi$ é logicamente válida, ou $=\phi$ simbolicamente, se $M \models \phi$ para toda $L$-estrutura $M$.

Prova-se por indução sobre a complexidade de $\phi\left(x_{1} \ldots x_{n}\right)$ que $a_{1} \ldots a_{n} \in M$ realizará $\phi\left(x_{1} \ldots x_{n}\right)$ em $M$ se, e somente se, $M_{M}$ for um modelo de $\phi\left(\underline{a_{1}} \ldots \underline{a_{n}}\right)$.

Teorema A.35. Seja $L^{\prime}$ uma assinatura que estende $L$, seja $M$ uma L-estrutura, seja $\phi\left(x_{1} \ldots x_{n}\right)$ uma L-fórmula, e sejam $a_{1} \ldots a_{n} \in M$. Se $M^{\prime}$ for uma expansão de $M a$ uma $L^{\prime}$-estrutura, então

$$
M \models \phi\left[a_{1} \ldots a_{n}\right] \Leftrightarrow M^{\prime} \models \phi\left[a_{1} \ldots a_{n}\right] .
$$

As $L$-estruturas podem ser classificadas de acordo com as $L$-sentenças que elas modelam.

Definição A.36. Sejam $M$ e $N$ duas $L$-estruturas. Dizemos que $M$ e $N$ são elementarmente equivalentes, ou $M \equiv N$ em símbolos, se

$$
M \models \phi \Leftrightarrow N \models \phi
$$

para qualquer $L$-sentença $\phi$.

6 Outro tipo fundamental de implicação é a noção de implicação sintática. Não trabalharemos com esse conceito nesta dissertação. 
Definição A.37. Uma classe $K$ de $L$-estruturas é dita ser axiomatizável (resp. finitamente axiomatizável) se existe um conjunto (resp. conjunto finito) $\Phi$ de $L$-sentenças tal que $K=\{M \vdots M \models \Phi\}$. Nesse caso, dizemos que $\Phi$ é um conjunto de axiomas para $K$.

Algumas classes de estruturas são automaticamente axiomatizáveis pela maneira que são definidas. Especificamente, dado um conjunto $\Phi$ de $L$-sentenças, se $K$ for definida como sendo a classe das $L$-estruturas que são modelos de $\Phi$, então $K$ será axiomatizável. Esse é o caso das classes dos grupos, dos aneis, dos corpos, dos corpos ordenados, etc.

\section{A.13 Teorias}

Se uma $L$-estrutura for modelo de um conjunto de $L$-sentenças, então ela também será modelo das $L$-sentenças que são implicações semânticas desse conjunto. Portanto, a classe das sentenças modeladas por uma estrutura é fechada sobre implicações semânticas, motivando a seguinte definição:

Definição A.38. Seja $T$ um conjunto de $L$-sentenças. Denotamos por $T^{\models}$ o conjunto das $L$-sentenças $\phi$ tais que $T \models \phi$. Dizemos que $T$ é uma $L$-teoria ou uma teoria em $L$ se $T=T^{\models}$. Os elementos de $T$ são chamados de teoremas de $T$.

Considere uma assinatura $L^{\prime}$, e suponha que há uma maneira de traduzir univocamente cada $L$-sentença para uma $L^{\prime}$-sentença, ou seja, existe uma função injetora $h: L_{\omega \omega}^{=} \rightarrow\left(L^{\prime}\right)_{\omega \omega}^{=}$. Assim, toda $L$-teoria $T$ estará em correspondência um-pra-um com o conjunto de $L^{\prime}$-sentenças $h\langle T\rangle$. Se $U$ for uma $L^{\prime}$-teoria com $h\langle T\rangle \subset U$, então a tradução de cada teorema de $T$ será um teorema de $U$, significando que $U$ estende $T$ de certa maneira, e se além disso tivermos $h^{-1}\langle U\rangle \subset T$, então a tradução inversa de cada teorema de $U$ que for inversamente traduzível será um teorema de $T$. Nesse caso, alguns fatores, como a expressividade da linguagem de $L^{\prime}$ e a força dedutiva dos axiomas que definem $U$, podem tornar mais vantajoso e conveniente trabalhar com a teoria $U$ que com $T$, mesmo que o interesse principal da discussão esteja voltado para os teoremas de $T$.

Definição A.39. Seja $L^{\prime}$ uma assinatura, seja $T$ uma $L$-teoria, seja $U$ uma $L^{\prime}$-teoria, e seja $h: L_{\omega \omega}^{=} \rightarrow\left(L^{\prime}\right)_{\omega \omega}^{=}$uma função injetora. Dizemos que $U$ é uma $h$-extensão conservativa de $T$ se $h\langle T\rangle \subset U$ e $h^{-1}\langle U\rangle \subset T$, ou seja, se a equivalência

$$
\phi \in T \Leftrightarrow h(\phi) \in U
$$

é verdadeira para qualquer $L$-sentença $\phi$. 
Existe uma função $h:\left(L_{\epsilon}\right)_{\omega \omega}^{=} \rightarrow\left(L_{\epsilon}\right)_{\omega \omega}^{=}$, a qual é definida indutivamente sobre a complexidade das $L_{\epsilon}$-sentenças em seu domínio, cuja aplicação $h(\phi)$ sobre uma $L_{\epsilon}$-fórmula $\phi$ consiste na troca de cada subfórmula de $\phi$ das formas $(\exists x) \chi$ e $(\forall x) \chi$ pelas respectivas fórmulas $\left(\exists^{c} x\right) \chi$ e $\left(\forall^{c} x\right) \chi$ (Definição B.3), mantendo o restante dos símbolos que ocorrem em $\phi$ intactos em suas posições originais. Prova-se que essa função é injetora e que o seguinte teorema é válido:

Teorema A.40. A $L_{\epsilon}$-teoria $N B G$ (Definição B.4) é uma h-extensão conservativa da $L_{\epsilon}$-teoria ZFC (Definição B.2).

\section{A.14 Subestruturas e Subestruturas Elementares}

Uma $L$-estrutura $N$ é substancialmente caracterizada por sua estabilidade - as coordenadas das relações $P^{N}$, os valores das funções $G^{N}$ e as constantes $c^{N}$ estão seguramente confinadas ao conjunto $\|N\|$, para qualquer símbolo relacional $P$ em $L$, qualquer símbolo funcional $G$ em $L$ e qualquer símbolo de constante $c$ em $L$. Se essa mesma sorte de estabilidade qualificar um subconjunto de $\|N\|$ perante as mesmas relações, funções e constantes que definem $N$, então esse subconjunto definirá uma $L$-estrutura de modo canônico.

Definição A.41. Seja $L$ uma assinatura e sejam $M$ e $N$ duas $L$-estruturas. Dizemos que $M$ é uma subestrutura de $N$, ou $M \subset N$ simbolicamente, se as seguintes condições são observadas:

(SubU) $\|M\| \subset\|N\| ;$

(SubR) $P^{M}=P^{N} \cap\|M\|^{n}$ para todo símbolo relacional $n$-ário $P$ em $L$;

(SubF) $G^{M}=G^{N} \uparrow\|M\|^{n}$ para todo símbolo funcional $n$-ário $G$ em $L$;

(SubC) $c^{M}=c^{N}$ para todo símbolo de constante $c$ em $L$.

Nesse caso, dizemos que $M$ é uma subestrutura elementar de $N$, ou $M \preccurlyeq N$ simbolicamente, se:

(SubEl) Para toda $L$-fórmula $\phi\left(x_{1} \ldots x_{n}\right)$ e para quaisquer $a_{1} \ldots a_{n} \in M$, temos

$$
M \models \phi\left[a_{1} \ldots a_{n}\right] \Leftrightarrow N \models \phi\left[a_{1} \ldots a_{n}\right] .
$$

\section{A.15 Morfismos Entre Estruturas}

A compreensão dos objetos matemáticos é substancialmente beneficiada quando examinamos a maneira que eles se relacionam entre si e buscamos assimilar as semelhanças e diferenças estruturais que eles manifestam, culminando na criação de variadas 
perspectivas para examinar os enigmas da disciplina. Na Teoria dos Modelos, as principais ferramentas de comparação entre estruturas estão definidas a seguir:

Definição A.42. Seja $L$ uma assinatura e sejam $M$ e $N$ duas $L$-estruturas.

- Uma função $f: M \rightarrow N$ é dita ser um morfismo de $M$ em $N$ se as seguintes condições são observadas:

(InvR) Para todo símbolo relacional $n$-ário $P$ em $L$ e para quaisquer $a_{1} \ldots a_{n} \in M$, temos

$$
\left(a_{1} \ldots a_{n}\right) \in P^{M} \Rightarrow\left(f\left(a_{1}\right) \ldots f\left(a_{n}\right)\right) \in P^{N}
$$

(InvF) Para todo símbolo funcional $n$-ário $G$ em $L$ e para quaisquer $a_{1} \ldots a_{n} \in M$, temos

$$
f\left(G^{M}\left(a_{1} \ldots a_{n}\right)\right)=G^{N}\left(f\left(a_{1}\right) \ldots f\left(a_{n}\right)\right) ;
$$

(InvC) Para todo símbolo de constante $c$ em $L$, temos $f\left(c^{M}\right)=c^{N}$.

Nesse caso, se $f$ for sobrejetora, então dizemos que $N$ é uma imagem homomórfica de $M$.

Se $f: M \rightarrow N$ for um morfismo, então temos as seguintes definições:

- Dizemos que $f$ é uma imersão se:

(Im) Para todo símbolo relacional $n$-ário $P$ em $L$ e para quaisquer $a_{1} \ldots a_{n} \in M$, temos

$$
\left(a_{1} \ldots a_{n}\right) \in P^{M} \Leftrightarrow\left(f\left(a_{1}\right) \ldots f\left(a_{n}\right)\right) \in P^{N} .
$$

Nesse caso, dizemos que $N$ é uma extensão de $M$;

- Dizemos que $f$ é uma imersão elementar se

(ImEl) Para toda $L$-fórmula $\phi\left(x_{1} \ldots x_{n}\right)$ e para quaisquer $a_{1} \ldots a_{n} \in M$, temos

$$
M \models \phi\left[a_{1} \ldots a_{n}\right] \Leftrightarrow N \models \phi\left[f\left(a_{1}\right) \ldots f\left(a_{n}\right)\right] .
$$

Nesse caso, dizemos que $N$ é uma extensão elementar de $M$;

- Dizemos que $f$ é um isomorfismo se $f$ é uma imersão sobrejetora. Nesse caso, dizemos que $M$ e $N$ são isomorfas, ou $M \cong N$ em símbolos.

Toda imersão é injetora e toda imersão elementar é uma imersão. Se existir uma imersão elementar $f: M \rightarrow N$, então $M \equiv N$. Prova-se que todo isomorfismo é uma imersão elementar e a função inversa de um isomorfismo é um isomorfismo.

Como a negação de uma condição da forma $M \models \phi$ é equivalente a $M \models(\neg \phi)$, temos que ( $\mathrm{ImEl}$ ) é equivalente à condição 
(ImEl') Para toda $L$-fórmula $\phi\left(x_{1} \ldots x_{n}\right)$ e para quaisquer $a_{1} \ldots a_{n} \in M$, temos

$$
M \models \phi\left[a_{1} \ldots a_{n}\right] \Rightarrow N \models \phi\left[f\left(a_{1}\right) \ldots f\left(a_{n}\right)\right] .
$$

Se $f$ for uma função $\|M\| \rightarrow\|N\|$ entre conjuntos que observa o axioma (ImEl'), então prova-se que $f$ será uma imersão elementar $M \rightarrow N$ entre $L$-estruturas. 



\section{B Formalizações da Teoria dos Conjuntos}

O estudo informal e intuitivo dos conjuntos, conhecido como Teoria Ingênua dos Conjuntos, provou ser incerto e pouco confiável à medida que diversos paradoxos foram sendo descobertos no início do século XX. O afastamento dessa abordagem foi encarado como uma prioridade crucial por vários pensadores, visto que praticamente todas as áreas da Matemática fundam-se decisivamente na noção de conjunto. A solução encontrada para esse problema consiste na instauração de uma série de postulados que determinam quais conjuntos podem ser considerados no estudo, tornando, assim, impraticável a construção dos conjuntos envolvidos nos paradoxos encontrados na versão ingênua do tema.

Várias axiomatizações da teoria foram propostas, a princípio com o mero objetivo de tornar o estudo consistente e posterioremente com outros objetivos variados, e as mais aceitas pela comunidade matemática são conhecidas como ZFC e NBG. A primeira trata exclusivamente de uma qualidade de objeto, os conjuntos, enquanto a segunda trata das classes, as quais são subdivididas em dois tipos: conjuntos e classes próprias. Ambas as teorias são equivalentes para teoremas que não envolvem classes próprias (Teorema A.40). Na visão do autor, NBG possibilita que muitos teoremas sejam apresentados de maneiras mais diretas e elegantes comparativamente à maneira que eles são exprimidos em ZFC, e, por tal razão, ela foi escolhida como metateoria para este trabalho.

Neste apêndice, apontaremos as definições de ZFC e NBG e evocaremos os teoremas de NBG que serão relevantes para a temática da dissertação. Para uma exposição detalhada, ver (39).

\section{B.1 As Teorias ZF, ZFC e NBG}

As teorias que veremos são descritas na linguagem de primeira ordem gerada pela assinatura $L_{\epsilon}$ (Exemplo A.7), onde o símbolo $\in$ representa a relação de pertinência.

Os pares ordenados podem ser manifestados em $L_{\epsilon}$-fórmulas que não têm quantificadores limitados (Definição A.16) assim como é indicado nas Notações A.30 e A.31, onde cada quantificador da forma $(\forall x \in U)$ deve ser substituído por $(\forall x)$. Deixaremos para o leitor a incumbência de especificar precisamente as representações das outras noções conjuntistas nas $L_{\epsilon}$-fórmulas, como as notações $x \subset y, x=\mathcal{P}(y)$ e $x \neq \emptyset$.

Nesta seção, denotaremos as variáveis de modo que símbolos distintos representem variáveis distintas na lista $v_{1} v_{2} \ldots v_{i} \ldots$ (Definição A.12). Ou seja, metavariáveis distintas representam variáveis distintas. 
Definição B.1. Chamamos de Teoria dos Conjuntos de Zermelo-Fraenkel, denominada sob a sigla $\mathbf{Z F}$, a $L_{\epsilon}$-teoria gerada pelos seguintes axiomas:

(Extensão) Se dois conjuntos tiverem os mesmos elementos, então eles serão iguais. Simbolicamente, temos

$$
(\forall X, Y)((\forall z)(z \in X \longleftrightarrow z \in Y) \longrightarrow X=Y)
$$

(Separação) Se $Z$ for um conjunto e $\phi$ for uma propriedade, então existirá o conjunto dos elementos de $Z$ que satisfazem à propriedade $\phi$. Simbolicamente, temos

$$
\left(\forall Z, w_{1} \ldots w_{n}\right)(\exists Y)(\forall x)(x \in Y \longleftrightarrow(x \in Z \wedge \phi))
$$

para qualquer $L_{\epsilon}$-fórmula $\phi\left(x, Z, w_{1} \ldots w_{n}\right)$;

(Par) Se $x$ e $y$ forem dois objetos, então existirá um conjunto que contém apenas $x$ e $y$ como elementos. Simbolicamente, temos

$$
(\forall x, y)(\exists Z) Z=\{x, y\}
$$

(União) Se $S$ for um conjunto, então existirá um conjunto $U$ que contém os elementos dos elementos de $S$. Simbolicamente, temos

$$
(\forall S)(\exists U)(\forall Y \in S)(\forall x \in Y) x \in U \text {; }
$$

(Substituição) Se $D$ for um conjunto e se $\phi\left(x, y, D, w_{1} \ldots w_{n}\right)$ for uma propriedade tal que para cada $x \in D$ existe um único objeto $y$ que torna a condição $\phi$ verdadeira, então existirá um conjunto $C$ tal que para cada $x \in D$ existe um $y \in C$ que torna $\phi$ verdadeira. Simbolicamente, temos

$$
\left(\forall D, w_{1} \ldots w_{n}\right)((\forall x \in D)(\exists ! y) \phi \longrightarrow(\exists C)(\forall x \in D)(\exists y \in C) \phi)
$$

para qualquer $L_{\epsilon}$-fórmula $\phi\left(x, y, D, w_{1} \ldots w_{n}\right)$;

(Infinito) Existe um conjunto $I$ que contém o conjunto vazio como elemento e é tal que $x \cup\{x\} \in I$ para todo $x \in I$. Simbolicamente, temos

$$
(\exists I)(\emptyset \in I \wedge(\forall x \in I) x \cup\{x\} \in I)
$$

(Potência) Se $X$ for um conjunto, então existirá um conjunto cujos elementos são os subconjuntos de $X$. Simbolicamente, temos

$$
(\forall X)(\exists Y) Y=\mathcal{P}(X)
$$


(Fundação) Se $X$ for um conjunto não nulo, então existirá um elemento $x$ de $X$ tal que todo elemento de $X$ não pertence a $x$. Simbolicamente, temos

$$
(\forall X)(X \neq \emptyset \longrightarrow(\exists x \in X)(\forall y \in X) y \notin x)
$$

Note que os Axiomas da Separação e da Substituição são esquemas axiomáticos, os quais descrevem infinitas $L_{\epsilon}$-fórmulas pertencentes a ZF. Prova-se que ZF não é finitamente axiomatizável (Definição A.37). Alguns autores não incluem o Axioma da Fundação na definição de ZF.

Denotaremos por Func $(f)$ a conjunção das $L_{\epsilon}$-fórmulas

- $(\forall z)(z \in f \longrightarrow(\exists x, y)(x, y)=z) ;^{1}$

- $\left(\forall x, y, y^{\prime}\right)\left(((x, y) \in f \wedge(x, y) \in f) \longrightarrow y=y^{\prime}\right)$

Essa fórmula simboliza que $f$ é uma função.

Definição B.2. Chamamos de Teoria dos Conjuntos de Zermelo-Fraenkel-Choice, denominada sob a sigla ZFC, a $L_{\epsilon}$-teoria gerada pelos axiomas de ZF e o axioma:

(Escolha) Se $X$ for um conjunto, então existirá uma função $f$ tal que qualquer subconjunto não nulo $S$ de $X$ pertence ao domínio de $f$ e é tal que $f(S) \in S$. Simbolicamente, temos

$$
(\forall X)(\exists f)[\text { Func }(f) \wedge(\forall S)((S \neq \emptyset \wedge S \subset X) \longrightarrow f(S) \in S)]
$$

Uma função $f$ definida em $\mathcal{P}(X)-\{\emptyset\}$ que satisfaz à propriedade descrita no Axioma da Escolha é dita ser uma função de escolha para $X$.

O Axioma da Escolha foi encarado com relutância e desaprovação por vários pensadores do século XX, em virtude dele ser utilizado para demonstrar a existência de alguns objetos matemáticos que não podem ser explicitamente construídos ${ }^{2}$ e implicar diversos resultados contraintuitivos e aparentemente contraditórios. No entanto, ele é quase unanimemente aceito pela comunidade matemática contemporânea, sendo supresso apenas em aplicações pontuais.

1 Essa fórmula simboliza que $f$ é uma relação.

2 Como exemplo, o Axioma da Escolha implica o Lema do Ultrafiltro (Teorema 3.42), o qual certifica a existência de ultrafiltros sem especificar os elementos que os compõem. 


\section{Definição B.3.}

- Denotaremos por $\mathrm{M}(x)$ a $L_{\epsilon}$-fórmula

$$
\mathrm{M}(x):=(\exists y)(x \in y) .
$$

Tal fórmula é lida como " $x$ é um conjunto", e a sua negação é lida como " $x$ é uma classe própria";

- Denotaremos por $\left(\forall^{\mathrm{c}} x\right) \phi$ a $L_{\epsilon}$-fórmula

$$
(\forall x)(\mathrm{M}(x) \rightarrow \phi)
$$

Tal fórmula é lida como " $\phi$ é verdadeira para todo conjunto $x$ ";

- Denotaremos por $\left(\exists^{\mathrm{c}} x\right) \phi$ a $L_{\epsilon}$-fórmula

$$
(\exists x)(\mathrm{M}(x) \wedge \phi) .
$$

Tal fórmula é lida como " $\phi$ é verdadeira para algum conjunto $x$ ".

O Axioma do Par implica que nenhum objeto é uma classe própria em ZF e ZFC.

Definição B.4. Chamamos de Teoria dos Conjuntos de Neumann-Bernays-Gödel, denominada sob a sigla NBG, a $L_{\epsilon}$-teoria gerada pelo Axioma da Extensão e os seguintes axiomas:

$\left(\operatorname{Par}^{c}\right)$ Se $x$ e $y$ forem dois conjuntos, então existirá um conjunto que contém apenas $x$ e y como elementos. Simbolicamente, temos

$$
\left(\forall^{\mathrm{c}} x, y\right)\left(\exists^{\mathrm{c}} Z\right)\left(\forall^{\mathrm{c}} u\right)(u \in Z \longleftrightarrow(u=x \vee u=y))
$$

(Conjunto Vazio) Existe um conjunto que não tem elemento algum. Simbolicamente, temos

$$
\left(\exists^{c} X\right)\left(\forall^{c} x\right) x \notin X
$$

(Relação de Pertinência) Existe uma classe cujos elementos são os pares ordenados $(x, y)$ de conjuntos tais que $x \in y$. Simbolicamente, temos

$$
(\exists E)\left(\forall^{\mathrm{c}} z\right)\left(z \in E \longleftrightarrow\left(\exists^{\mathrm{c}} x, y\right)(z=(x, y) \wedge x \in y)\right)
$$

(Interseção) Se $X$ e $Y$ forem duas classes, então existirá uma classe cujos elementos são os objetos que pertencem a $X$ e $Y$ simultaneamente. Simbolicamente, temos

$$
(\forall X, Y)(\exists Z)\left(\forall^{c} z\right)(z \in Z \longleftrightarrow(z \in X \wedge z \in Y))
$$


(Complemento) Se $X$ for uma classe, então existirá uma classe cujos elementos são os conjuntos que não pertencem a $X$. Simbolicamente, temos

$$
(\forall X)(\exists Y)\left(\forall^{c} z\right)(z \in Y \longleftrightarrow z \notin X)
$$

(Domínio) Se $X$ for uma classe, então existirá uma classe cujos elementos são os conjuntos que são primeiras coordenadas dos pares ordenados que pertencem a $X$. Simbolicamente, temos

$$
(\forall X)(\exists D)\left(\forall^{\mathrm{c}} u\right)\left(u \in D \longleftrightarrow\left(\exists^{\mathrm{c}} v\right)(u, v) \in X\right)
$$

(Relação Universal) Se $X$ for uma classe, então existirá uma classe cujos elementos são os pares ordenados de conjuntos tais que as primeiras coordenadas desses pares pertencem a $X$. Simbolicamente, temos

$$
(\forall X)(\exists Z)\left(\forall^{c} z\right)\left(z \in Z \longleftrightarrow(\exists x \in X)\left(\exists^{c} y\right) z=(x, y)\right)
$$

(Permutação 231) Se $X$ for uma classe, então existirá uma classe cujos elementos são as ternas ordenadas $(u, v, w)$ de conjuntos tais que a terna $(v, w, u)$ pertence a $X$. Simbolicamente, temos

$$
(\forall X)(\exists Z)\left(\forall^{\mathrm{c}} z\right)\left(z \in Z \longleftrightarrow\left(\exists^{\mathrm{c}} u, v, w\right)((v, w, u) \in X \wedge z=(u, v, w))\right)
$$

(Permutação 132) Se $X$ for uma classe, então existirá uma classe cujos elementos são as ternas ordenadas $(u, v, w)$ de conjuntos tais que a terna $(u, w, v)$ pertence a $X$. Simbolicamente, temos

$$
(\forall X)(\exists Z)\left(\forall^{c} z\right)\left(z \in Z \longleftrightarrow\left(\exists^{\mathrm{c}} u, v, w\right)((u, w, v) \in X \wedge z=(u, v, w))\right)
$$

(União $^{c}$ ) Se $X$ for um conjunto, então existirá um conjunto $U$ que contém os elementos dos elementos de $X$. Simbolicamente, temos

$$
\left(\forall^{c} X\right)\left(\exists^{\mathrm{c}} U\right)(\forall Y \in X)(\forall x \in Y) x \in U
$$

(Potência $^{c}$ ) Se $X$ for um conjunto, então existirá um conjunto cujos elementos são os subconjuntos de $X$. Simbolicamente, temos

$$
\left(\forall^{\mathrm{c}} X\right)\left(\exists^{\mathrm{c}} Y\right) Y=\mathcal{P}(X)
$$

(Subconjuntos) Se $X$ for um conjunto e se $Y$ for uma classe, então existirá um conjunto cujos elementos são os objetos que pertencem a $X$ e $Y$ simultaneamente. Simbolicamente, temos

$$
\left(\forall^{c} X\right)(\forall Y)\left(\exists^{c} Z\right)\left(\forall^{c} z\right)(z \in Z \longleftrightarrow(z \in X \wedge x \in Y))
$$


(Substituição $^{c}$ ) Se $f$ for uma função e se $X$ for um conjunto, então a imagem de $f$ sobre $X$ existirá como um conjunto. Simbolicamente, temos

$$
(\forall f)\left[\operatorname{Fnc}(f) \longrightarrow\left(\forall^{c} X\right)\left(\exists^{c} Y\right)\left(\forall^{c} y\right)(y \in Y \longleftrightarrow(\exists x \in X)(x, y) \in f)\right]
$$

$\left(\right.$ Infinito $\left.^{c}\right)$ Existe um conjunto $I$ que contém o conjunto vazio como elemento e é tal que $x \cup\{x\} \in I$ para todo $x \in I$. Simbolicamente, temos

$$
\left(\exists^{\mathrm{c}} I\right)(\emptyset \in I \wedge(\forall x \in I) x \cup\{x\} \in I)
$$

(Fundação $^{c}$ ) Se $X$ for um conjunto não nulo, então existirá um elemento $x$ de $X$ tal que todo elemento de $X$ não pertence a $x$. Simbolicamente, temos

$$
\left(\forall^{c} X\right)(X \neq \emptyset \longrightarrow(\exists x \in X)(\forall y \in X) y \notin x)
$$

$\left(\right.$ Escolha $\left.^{c}\right)$ Se $X$ for um conjunto, então existirá uma função $f$ tal que qualquer subconjunto não nulo $S$ de $X$ pertence ao domínio de $f$ e é tal que $f(S) \in S$. Simbolicamente, temos

$$
\left(\forall^{\mathrm{c}} X\right)\left(\exists^{\mathrm{c}} f\right)\left[\operatorname{Func}(f) \wedge\left(\forall^{\mathrm{c}} S\right)\left((S \neq \emptyset \wedge S \subset X) \longrightarrow\left(\exists^{\mathrm{c}} y \in S\right)(S, y) \in f\right)\right]
$$

Note que NBG é definida por dezessete axiomas, implicando que ela é uma $L_{\epsilon}$-teoria finitamente axiomatizável. Alguns autores não incluem o Axioma da Fundação na definição de NBG.

Como trataremos apenas de NBG no restante do trabalho, omitiremos o símbolo c sobrescrito no nome de alguns axiomas de NBG.

\section{B.2 Alguns Teoremas Básicos de NBG}

O Axioma dos Subconjuntos implica que todo subconjunto de um conjunto é um conjunto e o Axioma da Fundação implica no teorema a seguir:

Teorema B.5. Para cada conjunto $x$, temos $x \notin x$.

Demonstração. Considere o conjunto unitário $\{x\}$. Pelo Axioma da Fundação, existe um $a \in\{x\}$ tal que $y \notin a(\forall y \in x)$, implicando em $x \notin a$. Como $\{x\}$ é unitário e $a \in\{x\}$, temos $a=x$ e $x \notin x$.

Dizemos que uma $L_{\epsilon}$-fórmula $\phi$ é predicativa se ela pode ser escrita de maneira que todos os quantificadores que aparecem em $\phi$ sejam das formas $\left(\forall^{\mathrm{c}} x\right)$ e $\left(\exists^{\mathrm{c}} x\right)$, ou seja, se apenas quantificações sobre conjuntos aparecem em $\phi$. 
O Axioma da Relação de Pertinência atesta a existência da relação cujos elementos são os pares ordenados $(x, y) \operatorname{com} x \in y$, a qual será denotada por E. Utilizando-se de tal axioma, prova-se por indução sobre a complexidade das fórmulas que qualquer $L_{\epsilon}$-fórmula predicativa define uma classe.

Teorema B.6 (Teorema da Existência das Classes). Seja $\phi\left(x_{1} \ldots x_{m}, y_{1} \ldots y_{n}\right)$ uma $L_{\epsilon}$-fórmula predicativa. Para quaisquer classes $C_{1} \ldots C_{n}$, existe uma classe $Z$ tal que para quaisquer conjuntos $a_{1} \ldots a_{m}$ temos

$$
\left(a_{1} \ldots a_{m}\right) \in Z \Leftrightarrow \phi\left(a_{1} \ldots a_{m}, C_{1} \ldots C_{n}\right) .
$$

A classe $Z$ cuja existência é afirmada nesse teorema é denotada por

$$
Z=\left\{\left(x_{1} \ldots x_{m}\right) \vdots \phi\left(x_{1} \ldots x_{m}, C_{1} \ldots C_{n}\right)\right\} .
$$

Exemplo B.7. Se $X$ e $Y$ forem duas classes, então o Teorema da Existência das Classes nos permite definir a classe

$$
X \times Y:=\{(x, y) \vdots x \in X \wedge y \in Y\},
$$

a qual é chamada de produto cartesiano de $X$ e $Y$.

Exemplo B.8. Denotaremos por $\mathrm{V}$ a classe

$$
\mathrm{V}:=\{x: x=x\}
$$

a qual é conhecida como Universo de von Neumann.

Exemplo B.9. Para cada classe $X$, denotaremos por $\mathrm{E}_{X}$ a relação dada por

$$
\mathrm{E}_{X}:=\left\{\left(x_{1}, x_{2}\right) \vdots x_{1} \in x_{2} \wedge x_{1}, x_{2} \in X\right\}=\mathrm{E} \cap(X \times X) .
$$

Temos $\mathrm{E}_{\mathrm{V}}=\mathrm{E}$, e se $X$ for um conjunto, então $\mathrm{E}_{X}$ será um conjunto.

\section{B.3 Ordinais}

Existe uma maneira elegante e prática de listar todos os conjuntos bem ordenados, salvo isomorfismos. Os elementos dessa sequência de conjuntos comportam-se como números em diversos aspectos. 


\section{Definição B.10.}

- Dizemos que uma classe $X$ é transitiva se todo elemento de $X$ é um subconjunto de $X$;

- Dizemos que uma classe $\alpha$ é uma classe ordinal se $\alpha$ é transitiva e a relação $\mathrm{E}_{\alpha}$ é uma boa ordem ${ }^{3}$ em $\alpha$;

- Uma classe ordinal que é um conjunto é dita ser um ordinal. Os ordinais são comumente denotados por letras gregas minúsculas. A classe dos ordinais é denotada por On.

Exemplo B.11. Os conjuntos $\emptyset,\{\emptyset\},\{\emptyset,\{\emptyset\}\}$ e $\{\emptyset,\{\emptyset\},\{\emptyset,\{\emptyset\}\}\}$ são ordinais, respectivamente denotados por $0,1,2$ e 3 e chamados de zero, um, dois e três.

\section{Teorema B.12.}

- Se $X$ for uma classe ordinal, se $S \subsetneq X$, e se $S$ for transitivo, então $S \in X$;

- Se $X$ e $Y$ forem classes ordinais, então as condições $X \subsetneq Y$ e $X \in Y$ serão equivalentes;

- Se $X$ e $Y$ forem classes ordinais distintas, então ou $X \in Y$ ou $Y \in X$;

- Se $X$ for uma classe ordinal, então ou $X=$ On ou $X \in$ On;

- (Princípio da Boa Ordenação) A classe própria $\mathrm{E}_{\mathrm{On}}$ é uma boa ordem em On;

- On é a única classe ordinal que é uma classe própria;

- Se $X$ for uma classe não nula de ordinais, então $\bigcap X$ será o menor elemento de $X$ em On;

- Se X for um conjunto de ordinais, então $\bigcup X$ será o supremo de $X$ em On.

Para quaisquer ordinais $\alpha$ e $\beta$, a condição $\alpha \in \beta$ é denotada por $\alpha<\beta$, uma vez que $E_{\text {On }}$ é uma ordem em On.

Os ordinais satisfazem a um tipo extremamente útil de indução muito utilizado em diversas áreas da Matemática.

Teorema B.13 (Princípio da Indução Transfinita - Primeira Versão). Seja X uma classe. Se para todo ordinal $\beta$ a condição $\beta \subset X$ implicar $\beta \in X$, então On $\subset X$.

3 Uma ordem < em uma classe $X$ é dita ser uma boa ordem em $X$ se qualquer subclasse não nula de $X$ tiver um menor elemento. Nesse caso, dizemos que $X$ é bem ordenado por $<$. 
Cada ordinal $\alpha$ é um conjunto bem ordenado quando munido da ordem $\mathrm{E}_{\alpha}$ em $\alpha$. Reciprocamente, cada conjunto bem ordenado é isomorfo a exatamente um ordinal.

Teorema B.14. Seja < uma boa ordem em um conjunto $X$. Existem um único ordinal $\alpha$ e uma única função $f: X \rightarrow \alpha$ tais que $f$ é um isomorfismo entre os conjuntos ordenados $(X,<) e\left(\alpha, \mathrm{E}_{\alpha}\right)$.

Dado um ordinal $\alpha$, podemos definir um conjunto bem ordenado a partir de $\alpha$ adicionando a este um elemento maior que todo elemento contido nele. Constata-se que tal conjunto ordenado será um ordinal quando o elemento adicionado a $\alpha$ for o próprio $\alpha$.

Definição B.15. Se $\alpha$ for um ordinal, então o ordinal $\alpha \cup\{\alpha\}$ será chamado de sucessor de $\alpha$ e será denotado por $\alpha^{\prime}$. Nesse caso, dizemos que $\alpha$ é o antecessor de $\alpha^{\prime}$. Um ordinal não zero $\alpha$ é dito ser um ordinal sucessor, ou Suc $(\alpha)$ simbolicamente, se ele é o sucessor de algum ordinal, e caso contrário dizemos que ele é um ordinal limite.

O sucessor de um ordinal é o menor ordinal que o supera.

Teorema B.16. Sejam $\alpha$ e $\beta$ dois ordinais.

- Um conjunto $X$ será um ordinal se, e somente se, o conjunto $X^{\prime}:=X \cup\{X\}$ for um ordinal;

- Não existe um ordinal $\gamma$ tal que $\alpha<\gamma<\alpha^{\prime}$;

- Se $\alpha^{\prime}=\beta^{\prime}$, ent $\tilde{a} o \alpha=\beta$;

- Teremos $\alpha<\beta^{\prime}$ se, e somente se, $\alpha \leqslant \beta$.

Teorema B.17 (Princípio da Indução Transfinita - Segunda Versão). Seja X uma classe. Se forem observadas as condições

- $0 \in X$;

- Se $\alpha$ for um ordinal em $X$, então $\alpha^{\prime} \in S$;
- Se $\beta$ for um ordinal limite, e se $\beta \subset X$, então $\beta \in X$,

então On $\subset X$.

Definição B.18. O conjunto dos ordinais finitos é a classe $\omega$ definida por

$$
\omega:=\{n \vdots(n=0 \vee \operatorname{Suc}(n)) \wedge(\forall x \in n)(n=0 \vee \operatorname{Suc}(x))\} .
$$

Dizemos que os ordinais não pertencentes a $\omega$ são infinitos. 
A classe $\omega$ é um conjunto, e temos $0,1,2,3 \in \omega$ (Exemplo B.11).

Teorema B.19. Sejam $\alpha$ e $\beta$ dois ordinais.

- $\alpha \in \omega$ se, e somente se, $\alpha^{\prime} \in \omega$;

- $\omega$ é um ordinal limite.

- Se $\alpha \in \omega$ e $\beta<\alpha$, então $\beta \in \omega$;

Exemplo B.20. Se $X$ for um conjunto de ordinais com $0 \in X$ tal que o sucessor de cada ordinal finito em $X$ pertence a $X$, então, utilizando-se do Axioma do Infinito, prova-se que todo ordinal finito pertencerá a $X$. Essa conclusão é conhecida como Princípio da Indução.

O teorema a seguir descreve uma técnica poderosa de construção de sequências com índices em On tais que cada elemento é determinado ou pelo seu antecessor ou por todos os elementos que aparecem antes dele na sequência.

Teorema B.21 (Recursão Transfinita). Seja $x$ um conjunto, seja $S: \mathrm{V} \rightarrow \mathrm{V}$ uma função, e seja L uma função cujo domínio é a classe das funções $f$ com dom $(f) \in$ On. Existe uma única função $F: \mathrm{On} \rightarrow \mathrm{V}$ que satisfaz às seguintes condições:

- $F(0)=x$;

- $F\left(\alpha^{\prime}\right)=S(F(\alpha))$ para cada ordinal $\alpha$
- $F(\alpha)=L\left(F \uparrow_{\alpha}\right) \quad$ para cada ordinal limite $\alpha$.

Várias operações entre os ordinais, como a adição, a multiplicação, a exponenciação, etc. podem ser definidas via Recursão Transfinita. Neste apêndice, mostraremos apenas o caso da adição em On.

Exemplo B.22. Seja $\alpha$ um ordinal e seja $F_{\alpha}:$ On $\rightarrow$ On a função definida recursivamente (Teorema B.21) pelas seguintes condições:

- $F_{\alpha}(0):=\alpha$;

- $F_{\alpha}(\beta):=\bigcup_{\gamma<\beta} F_{\alpha}(\gamma)$ para cada ordinal - $F_{\alpha}\left(\beta^{\prime}\right):=\left(F_{\alpha}(\beta)\right)^{\prime}$ para cada ordinal $\beta$; limite $\beta$.

Para cada ordinal $\beta$, a imagem $F_{\alpha}(\beta)$ é dita ser a soma dos ordinais $\alpha$ e $\beta$, e ela é denotada por $\alpha+\beta$. A função $+:$ On $\times$ On $\rightarrow$ On dada por $(\alpha, \beta) \mapsto F_{\alpha}(\beta)$ é chamada de adição em On.

É comum que muitas propriedades das funções definidas por Recursão Transfinita sejam demonstradas por intermédio do Princípio da Indução Transfinita. 
Teorema B.23. Para quaisquer ordinais $\alpha, \beta$ e $\gamma$, temos:

- 0 é um elemento neutro de +;

- $\alpha+1=\alpha^{\prime}$

- + é associativa mas não é comutativa;

- $+\lceil\omega \times \omega$ é a adição usual em $\omega ;$

O processo de contagem consiste em efetuar correspondências um-para-um entre os elementos de duas classes. Desse modo, pode-se estabelecer uma comparação precisa entre os "tamanhos" das classes sem sequer atribuir uma escala para mensurar essa grandeza.

Definição B.24. Sejam $X$ e $Y$ duas classes.

- Denotamos $X \preccurlyeq Y$ se existir uma função injetora do tipo $X \rightarrow Y$;

- Dizemos que $X$ e $Y$ são equipotentes, ou $X \cong Y$ simbolicamente, se existe uma função bijetora do tipo $X \rightarrow Y$;

- Denotamos $X \prec Y$ se $X \preccurlyeq Y$ e $X \neq Y$;

- Um conjunto é dito ser finito se ele é equipotente a um ordinal finito, e caso contrário ele é dito ser infinito;

- Dizemos que um conjunto é enumerável se ele é equipotente a $\omega$;

- Dizemos que um conjunto é contável se ele é finito ou enumerável.

A classe dos pares de conjuntos $(X, Y)$ tais que $X \cong Y$ é uma relação de equivalência em $V$, a qual é chamada de relação de equipotência entre conjuntos.

Teorema B.25. Sejam X, Y e Z classes.

- $X \preccurlyeq X$ e $X \nprec X$;

- Se $X \subset Y$, então $X \preccurlyeq Y$;

- Se $X \preccurlyeq Y$ e $Y \preccurlyeq Z$, ent $\tilde{a} o \quad X \preccurlyeq Z$;

- (Teorema de Bernstein) Se $X \preccurlyeq Y$ e $Y \preccurlyeq X$, então $X \cong Y$.

- Se $\alpha$ for um ordinal infinito, então $\alpha \cong \alpha^{\prime}$;

- Se $\alpha$ for um ordinal finito, $\beta$ for um ordinal, e $\alpha \cong \beta$, então $\alpha=\beta$; 
- Se $\alpha$ for um ordinal finito, e $x \subsetneq \alpha$, então $x \neq \alpha$;

- (Teorema de Hartog) Se X for um conjunto, então existirá um ordinal a que não é equipotente a qualquer subconjunto de $X$.

Algumas condições equivalentes ao Axioma da Escolha são bastante aproveitadas nas demonstrações dos teoremas de NBG.

Teorema B.26. Seja $T$ a $L_{\epsilon}$-teoria gerada pelos axiomas de $N B G$ exceto o Axioma da Fundação e o Axioma da Escolha. As seguintes condições são equivalentes ao Axioma da Escolha módulo T:

- Produtos cartesianos de famílias de conjuntos não nulos são não nulos;

- (Teorema de Zermelo) Todo conjunto pode ser bem ordenado;

- Para quaisquer conjuntos $X$ e $Y$, temos $X \preccurlyeq Y$ ou $Y \preccurlyeq X$;

- (Lema de Zorn) Se toda cadeia ordenada em um conjunto parcialmente ordenado tiver uma cota superior, esse conjunto parcialmente ordenado terá um elemento maximal.

As equivalências retratadas no Teorema B.26 também são válidas módulo a $L_{\epsilon}$-teoria gerada pelos axiomas de ZF exceto o Axioma da Fundação.

\section{B.4 Cardinais}

Se $R$ for a relação de equipotência entre dois ordinais, então cada classe de equivalência no quociente On/ $R$ representará uma "quantidade" de elementos que um conjunto pode compreender. Em virtude da classe On ser bem ordenada por $E_{O n}$, uma maneira natural de apontar um representante para cada classe de equivalência em On $/ R$ consiste em escolher o menor ordinal pertencente a cada classe.

Definição B.27. Seja $X$ um conjunto e seja $\alpha$ um ordinal.

- Dizemos que um ordinal é um cardinal ou um ordinal inicial se, e somente se, ele não é equipotente a um ordinal menor que ele;

- Pelo Teorema de Zermelo (Teorema B.26) e pelo Teorema B.14, existe um ordinal equipotente a $X$. O menor ordinal equipotente a $X$ é um cardinal, o qual é chamado de cardinal de $X$ e é denotado por $|X|$;

4 Uma cadeia ordenada em um conjunto parcialmente ordenado $(E,<)$ é um subconjunto $S$ de $E$ tal que $x<y$ ou $y<x$ para quaisquer $x, y \in S$ distintos. 
- Pelo Teorema de Hartog (Teorema B.25), existe um ordinal maior que $\alpha$ que não é equipotente a $\alpha$. O menor ordinal maior que $\alpha$ que não é equipotente a $\alpha$ é um cardinal, o qual é chamado de cardinal sucessor de $\alpha$ e é denotado por $\alpha^{+}$.

Todo ordinal finito é um cardinal, e todo cardinal infinito é um ordinal limite. Temos $n^{+}=n+1(\forall n \in \omega)$, e se $\alpha$ for um cardinal, então $|\alpha|=\alpha$.

Sem o Axioma da Escolha, não seria possível atribuir um cardinal $\alpha$ a cada conjunto $X$ de modo que $X \cong \alpha$.

Exemplo B.28. O ordinal $\omega$ é o cardinal dos conjuntos numéricos $\mathbb{N}, \mathbb{Z}$ e $\mathbb{Q}$.

Seja $^{5}\left\{\omega_{\gamma}\right\}_{\gamma \in \text { On }}$ a sequência definida recursivamente (Teorema B.21) pelas seguintes condições:

- $\omega_{0}:=\omega ; \quad$ - $\omega_{\alpha}=\bigcup_{\gamma<\alpha} \omega_{\gamma}$ para cada ordinal limite $\alpha$.

- $\omega_{\alpha^{\prime}}=\left(\omega_{\alpha}\right)^{+}$para cada ordinal $\alpha$;

Teorema B.29. Sejam $X$ e $Y$ dois conjuntos.

- $\left\{\omega_{\alpha}\right\}_{\alpha \in \mathrm{On}}$ é a classe dos cardinais infinitos;

- A função $f:$ On $\rightarrow\left\{\omega_{\alpha}\right\}_{\alpha \in \mathrm{On}}$ definida por $f(\alpha):=\omega_{\alpha}$ é um isomorfismo entre classes ordenadas;

- $\alpha \leqslant \omega_{\alpha}$ para todo ordinal $\alpha$;

- Teremos $|X|=|Y|$ se, e somente se, existir uma função bijetora do tipo $X \rightarrow Y$;

- (Princípio da Casa dos Pombos) Se $|Y|<|X|$, então toda função sobrejetora do tipo $X \rightarrow Y$ não será injetora;

- (Teorema de Cantor) $|X|<|\mathcal{P}(X)|$.

\section{B.5 Hierarquia Cumulativa dos Conjuntos}

Seja $V_{0} \bigvee_{1} \ldots V_{\omega} \ldots V_{\alpha} \ldots$ a sequência definida por Recursão Transfinita (Teorema B.21) da seguinte maneira:

- $\mathrm{V}_{0}:=\emptyset$

- $\mathrm{V}_{\alpha}:=\bigcup_{\gamma<\alpha} \mathrm{V}_{\gamma}$ para cada ordinal limite $\alpha$.

- $\mathrm{V}_{\alpha^{\prime}}:=\mathrm{V}_{\alpha} \cup \mathcal{P}\left(\mathrm{V}_{\alpha}\right)$ para cada ordinal $\alpha$;

$\overline{5}$ Muitos autores denotam essa sequência por $\left\{\aleph_{\gamma}\right\}_{\gamma \in \text { On }}$. 
Tal sequência é dita ser a hierarquia cumulativa dos conjuntos em NBG. Para cada $n \in \omega$, temos que $\mathrm{V}_{n}$ é um conjunto finito de conjuntos finitos. Em particular, todo elemento de $\mathrm{V}_{\omega}$ é finito.

\section{Teorema B.30.}

- $\mathrm{V}_{\alpha} \subsetneq \mathrm{V}_{\beta}$ se, e somente se, $\alpha<\beta ; \quad$ - $\mathrm{V}_{\alpha}=\bigcup_{\gamma<\alpha} \mathcal{P}\left(\mathrm{V}_{\gamma}\right) \quad$ para $\quad$ cada ordinal - $\mathrm{V}_{\alpha^{\prime}}=\mathcal{P}\left(\mathrm{V}_{\alpha}\right)$ para cada ordinal $\alpha ; \quad$ limite $\alpha$.

O Axioma da Fundação permite que os conjuntos em NBG sejam plenamente hierarquizados de acordo com o menor nível que os contêm na sequência dos $V_{\alpha}$.

Teorema B.31. Seja $T$ a $L_{\epsilon}$-teoria gerada pelos axiomas de $N B G$ exceto o Axioma da Fundação e o Axioma da Escolha. O Axioma da Fundação é equivalente à equação $\mathrm{V}=\bigcup_{\alpha \in \mathrm{On}} \mathrm{V}_{\alpha}$ módulo $T$.

Definição B.32. Seja $X$ um conjunto. O menor ordinal $\alpha$ tal que $X \in \mathrm{V}_{\alpha^{\prime}}$ é chamado de posto de $X$ e é denotado por posto $(X)$.

Todo conjunto infinito tem posto infinito.

Proposição B.33.

$$
\operatorname{posto}(X)=\sup \{\operatorname{posto}(x)+1 \vdots x \in X\} .
$$

Exemplo B.34. Como $\emptyset \in \mathrm{V}_{0^{\prime}}=\mathcal{P}\left(\mathrm{V}_{0}\right)$, temos posto $(\emptyset)=0$. Prova-se por indução transfinita que posto $(\alpha)=\alpha(\forall \alpha \in \mathrm{On})$, implicando em $\alpha \in \mathrm{V}_{\alpha^{\prime}}(\forall \alpha \in \mathrm{On})$.

Exemplo B.35. Temos posto $\left(\mathrm{V}_{\alpha}\right)=\alpha(\forall \alpha \in \mathrm{On})$.

Corolário B.36. Se $X$ e $Y$ forem dois conjuntos tais que $X \in Y$, então

$$
\text { posto }(X)<\operatorname{posto}(Y)
$$




\section{Referências}

1 WIKIPEDIA. Archimedean property. Wikipedia, 2017. Disponivel em:

$<$ https://en.wikipedia.org/w/index.php?title=Archimedean_property\&oldid $=799010700>$.

Acesso em: 26 de Fevereiro de 2018.

2 BAIR, J. et al. Is Mathematical History Written by the Victors? Notices of the American Mathematical Society, v. 60, p. 886-904, 2013.

3 BASCELLI, T. et al. Fermat, Leibniz, Euler, and the gang: The True History of the Concepts of Limit and Shadow. Notices of the American Mathematical Society, v. 61, no. 8, 2014.

4 BELL, J. L. The Continuous and the Infinitesimal in Mathematics and Philosophy. Polimetrica, International Scientific Publisher, 2008. 354 p.

5 BERKELEY, G. The Analyst. Calgary, Alberta, CA: Theophania Publishing, 2011. $60 \mathrm{p}$.

6 BLASZCZYK, P. et al. Toward a history of mathematics focused on procedures. Foundations of Science, p. 30, 2016.

7 BLASZCZYK, P.; KATZ, M. G.; SHERRY, D. Ten Misconceptions from the History of Analysis and Their Debunking. Foundations of Science, p. 46, 2012.

8 BOROVIK, A.; KATZ, M. G. Who Gave You the Cauchy-Weierstrass Tale? The Dual History of Rigorous Calculus. Foundations of Science, v. 17, n. 3, p. 245-276, Agosto de 2011.

9 H. J. M. BOS. Differentials, higher-order differentials and the derivative in the Leibnizian calculus. Archive for History of Exact Sciences, v. 14, n. 1, p. 1-90, Março de 1974.

10 BOYER, C. B. The History of the Calculus and Its Conceptual Development. 1. ed. [S.l.]: Dover Publications, 1959. 368 p.

11 CAJORI, F. A History of Mathematical Notations. [S.l.]: Cosimo Classics, v. 2, 2007. 392 p.

12 CAUCHY, A. L. Cours d'analyse de l'École royale polytechnique. [S.l.]: [Paris] Imprimerie royale, 1821. $612 \mathrm{p}$.

13 CHANG, C. C.; KEISLER, H. J. Model Theory. 3. ed. [S.l.]: Dover Publications, $672 \mathrm{p}$. 
14 COHEN, L. W.; EHRLICH, G. The Structure of the Real Number System.

Proceedings of the Edinburgh Mathematical Society, Edinburgh, v. 15, n. 2, p. 159-160, 1963.

15 COLlinS, J. Commercium epistolicum D. Johannis Collins, et aliorum, de analysi promota. [S.l.]: Gale ECCO, 2010. 270 p. Edição em Latin.

16 CONWAY, J. H. On Numbers and Games. 1. ed. [S.1.]: A K Peters/CRC Press , 2000. $252 \mathrm{p}$.

17 DAUBEN, J. W. Georg Cantor: His Mathematics and Philosophy of the Infinite. 1. ed. [S.1.]: Princeton University Press, 1990. 404 p.

18 DAVIS, M. The Universal Computer: The Road from Leibniz to Turing. 1. ed. [S.l.]: W. W. Norton \& Company, 2000. 256 p.

19 APPLIED Nonstandard Analysis. [S.l.]: Dover Publications, 2005. 208 p.

20 EHRLICH, P. (Ed.). Real Numbers, Generalizations of the Reals, and Theories of Continua. 1. ed. [S.l.]: Springer Netherlands, v. 242, 1994. 288 p.

21 EUlER, L. Foundations of Differential Calculus. Tradução de J. D. Blanton. [S.l.]: Springer, 2000. 194 p.

22 Advances in Non-Archimedean Analysis. 13th International Conference on p-adic Functional Analysis. Paderborn: American Mathematical Society. 2014.

23 GOLDBLATT, R. Lectures on the Hyperreals: An Introduction to Nonstandard Analysis. 1. ed. [S.1.]: Springer-Verlag New York, 1998. 293 p.

24 GONSHOR, H. An Introduction to the Theory of Surreal Numbers. 1. ed. [S.1.]: Cambridge University Press, 1986. 204 p. London Mathematical Society Lecture Note Series (Book 110).

25 GRAY, J. The Real and the Complex: A History of Analysis in the 19th Century. 1. ed. [S.l.]: Springer International Publishing, 2015. 350 p. Springer Undergraduate Mathematics Series.

26 HALL, A. R. Philosophers at War: The Quarrel between Newton and Leibniz. [S.l.]: Cambridge University Press, 2002. 356 p.

27 HEWITT, E. Rings of Real-Valued Continuous Functions. I. Transactions of the American Mathematical Society, v. 64, n. 1, p. 45-99, Julho de 1948.

28 HODGES, W. Model Theory. 1. ed. [S.1.]: Cambridge University Press, 2008. 778 p. Encyclopedia of Mathematics and its Applications (Book 42). 
29 KATZ, M. G.; TALL, D. Tension between Intuitive Infinitesimals and Formal Mathematical Analysis. Crossroads in the History of Mathematics and Mathematics Education. The Montana Mathematics Enthusiast Monographs in Mathematics Education, v. 12, p. 19, 26 de Outubro de 2011.

30 KATZ, M. G.; SHERRY, D. Leibniz's Infinitesimals: Their Fictionality, Their Modern Implementations, and Their Foes from Berkeley to Russell and Beyond. Erkenntnis, v. 78, n. 3, p. 571-625.

31 KNUTH, D. E. Surreal Numbers. 1. ed. [S.l.]: Addison-Wesley Professional, 1974. $119 \mathrm{p}$.

32 LAGRAnGE, J. L.; BINET, J. P. M.; GARNIER, J. G. Mécanique analytique. [S.1.]: Paris, Ve Courcier, v. 2, 1811. 784 p.

33 LANG, S. Algebra. 3. ed. [S.l.]: Springer-Verlag New York, v. 211, 2002. 914 p. Graduate Texts in Mathematics.

34 LEIBNIZ, G. W. Leibniz: Philosophical Essays (R. Ariew \& D. Garber, Trads.). 1st edition ed. Indianapolis: Hackett Publishing Company, 1989.

35 LEIBNIZ, G. W. The Early Mathematical Manuscripts of Leibniz. Tradução de J. M. Child. [S.1.]: Dover Publications, 2005. 256 p.

36 LOEB, P. A.; WOLFF, M. P. H. (Eds.). Nonstandard Analysis for the Working Mathematician. 2. ed. [S.l.]: Springer Netherlands, 2015. 481 p.

37 LUXEMBURG, W. A. J. Two applications of the method of construction by ultrapowers to analysis. Bulletin of the American Mathematical Society, v. 68, n. 4, p. 416-419, 4 de Julho de 2007.

38 LUXEMBURG, W. A. J. Non-Standard Analysis: Lectures on A. Robinson's Theory of Infinitesimals and Infinitely Large Numbers. 2. ed. [S.l.]: [s.n.], 1964. (Recurso para Ensino; Não Publicado).

39 MENDELSOHN, E. Introduction to Mathematical Logic. 1. ed. [S.1.]: Springer US, 1987. $342 \mathrm{p}$.

40 MOORE, G. H. Zermelo's Axiom of Choice: Its Origins, Development, and Influence. 1. ed. [S.l.]: Springer-Verlag New York, v. 8, 1982. 412 p.

41 NOETHER, E. Abstrakter Aufbau der Idealtheorie in algebraischen Zahl- und Funktionenkörpern. Mathematische Annalen, v. 96, p. 26-61, 1927.

42 PINCUS, D.; SOLOVAY, R. M. Definability of measures and ultrafilters. The Journal of Symbolic Logic, v. 42, n. 2, p. 179-190, Junho de 1977. 
43 PINTO, J. S. Infinitesimal Methods of Mathematical Analysis. 1. ed. [S.l.]: Woodhead Publishing, 2004. 270 p.

44 RESCHER, N. Leibniz's Monadology: An Edition for Students. 1. ed. [S.l.]: University of Pittsburgh Press, 1991. 480 p.

45 ROBINSON, A. Non-standard Analysis. [S.l.]: Princeton University Press, 1996. 308 p.

46 ROBINSON, A.; ZAKON, E. A set theoretical characterization of enlargements: Applications of model theory to algebra, analysis and probability. International Symposium, Pasadena (CA): Rinehard \& Winston. 1967. p. 109-122.

47 RUSSELL, B. A critical exposition of the philosophy of Leibniz, with an appendix of leading passages. [S.1.]: Nabu Press, 2010. 336 p.

48 SHAMSEDDINE, K.; BERZ, M. Analysis on the Levi-Civita field: A brief overview. Contemporary Mathematics, v. 508, p. 215-237, 2010.

49 SHAMSEDDINE, K. New results on integration on the Levi-Civita field. Indagationes Mathematicae, v. 24, n. 1, p. 199-211, 2013.

50 STROYAN, K. D.; LUXEMBURG, W. A. J. Introduction to the theory of infinitesimals. New York: New York Academic Press, 1976. 326 p.

51 STRUIK, D. J. A Source Book in Mathematics, 1200-1800. [S.l.]: Princeton University Press, 2014. 444 p.

52 VÄTH, M. A. Nonstandard Analysis. [S.l.]: Birkhäuser, 2007. 252 p.

53 ŁOŚ, J. Quelques remarques, théorèmes et problèmes sur les classes définissables d'algèbres. Mathematical interpretation of formal systems. North-Holland Publishing Co., Amsterdam, p. 98-113, 1955. Studies in logic and the foundations of mathematics.

54 ZAKON, E. A new variant of non-standard analysis. Victoria Symposium on Nonstandard Analysis. Springer, Berlin, Heidelberg. 1974. p. 313-339. Lecture Notes in Mathematics. 


\section{Îndice}

A

Adição em On, 180

Alfabeto de uma assinatura, 154

Antecessor de um ordinal sucessor, 179

Apreciável

elemento, 12

Aridade, 153

Assinatura, 152

dos anéis, 154

dos anéis ordenados, 154

dos conjuntos, 153

dos grupos, 153

vazia, 153

Átomo

em $X_{\omega}, 106$

relacionado a $* \mathbb{R}, 36$

relacionado a $\mathbb{R}, 35$

Axioma, 166

da Escolha, 64, 98, 133, 173, 176

da Escolha Contável, 64

Axiomatizável

classe de estruturas, 166

B

Base

conjunto, 107

C

Canônica

função, 84

Cardinal, 182

de um conjunto, xix, 182

interno, 127

Cauchy

sequência de, 27

Cauchy-completo

domínio ordenado, 27
Classe, xv, 171

de índices, xvi

ordinal, 178

própria, 174

transitiva, 178

Cofinito

subconjunto, 77

Completo

filtro, 98

Complexidade

de um termo, 156

de uma fórmula, 156

Composição de relações, xviii

Condição, xv

aberta, 38

completamente aberta, 38

fechada, 38

negação de uma, xv

relacionada a $* \mathbb{R}, 38$

relacionada a $\mathbb{R}, 38$

Condição fechada

falsa, 38

verdadeira, 38

Condições

conjunção de, xv

definição de, xv

equivalência de, xv

implicação de, xv

Confrontante

relação de equivalência, 15

Conjunto, 171, 174

das partes, xvi

em $X_{\omega}, 106$

potência, xvi

relacionado a $* \mathbb{R}, 36$

relacionado a $\mathbb{R}, 35$ 
vazio, $x v i$

Construção por ultraprodutos, 146

Contagem, 181

Contável

conjunto, 181

Convergente

sequência, 27

Corpo

das frações de $\mathbb{R}[x], 9$

das funções racionais, 9

dos números hiper-reais, 101

não estritamente ordenado, 8

ordenado, 8

ordenado das séries de Laurent, 11

Corte de Dedekind, 24

Cota

inferior, 21

superior, 21

\section{D}

Dedekind-completo

domínio ordenado, 24

Diferença, xvi

Disjunção inclusiva, xv

Divisão, 8

Domínio

arquimediano, 13

não arquimediano, 13

não estritamente ordenado, 3

ordenado, 1

Dupla implicação, 157

E

Enumerável

conjunto, 181

Equipotentes

classes, 181

Equivalência, 157

Esquema de substituição, 152

Estrutura, 160
Estruturas

elementarmente equivalentes, 165

isomorfas, 168

Expansão de uma estrutura, 161

Extensão

conservativa, 166

de uma assinatura, 154

de uma estrutura, 168

elementar, 168

Externo

objeto, 47,114

F

Família, xvi

Filtro, 76

das caudas, 79

de Fréchet, 77

dos cofinitos, 77

impróprio, 76

livre, 76

maximal, 76

não principal, 76

principal, 76

próprio, 76

trivial, 76

Finitamente axiomatizável classe de estruturas, 166

Finitamente próximos

elementos, 19

Finito

conjunto, 181

elemento, 11

Fórmula, 156

atômica, 156

com quantificadores limitados, 157

logicamente válida, 165

predicativa, 176

Função, xix, 173

de Escolha, 173

identidade, xix 
G

Galáxia de um elemento, 19

$\mathbf{H}$

Hierarquia cumulativa, 184

Hiperfinito

conjunto, 124

operação, 129

produto, 68, 129

soma, 65

Hiperpartição, 71

refinada, 71

Hipersequência, 53, 65

\section{I}

Ideal ordenado, 17

Imagem homomórfica, 168

Imersão, 168

elementar, 168

Ímpar

número hiperinteiro, 45

Implicação, 157

Implicação semântica, 165

Inclusão

estrita, xv

não estrita, xv

Incompleto

filtro, 98

Ínfimo, 21

Infinitamente próximos

elementos, 19

Infinitesimal

elemento, 11

em relação a outro elemento, 11

Infinito

conjunto, 181

elemento, 11

Interno

objeto, 47, 114

Interpretação de um símbolo de constante, 160

de um símbolo funcional, 160

de um símbolo relacional, 160

de um termo, 163

Interseção, xvi

enumerável, xviii

Intervalo aberto, xix

Isomorfismo, 168

K

Kernel, 3

L

Lacuna, 24

Lema

de Zorn, 182

do Ultrafiltro, 92

Sequencial de Robinson, 131

Limite

de uma sequência convergente, 27

em um produto reduzido, 82

funcional, 88

integral, 86

relacional, 87

Linguagem, 152

de primeira ordem, 156

M

Maior cota inferior, 21

Maior elemento, 21

Menor cota superior, 21

Menor elemento, 21

Modelo

de um conjunto de fórmulas, 165

de uma fórmula, 165

Módulo, 7

Mônada de um elemento, 19

Monóide livre, 151

Monomorfismo não standard, 39, 111

Morfismo, 168 
Não negativo

elemento, 2

Não positivo

elemento, 2

Não standard

objeto, 39, 114

Negativo

elemento, 2

Nível

em $\mathbb{I}^{\omega}, 114$

em $\mathbb{I}_{\omega}, 114$

em $X^{\omega}, 106$

em $X_{\omega}, 106$

Número

hiperinteiro, 44, 102

hiperirracional, 44, 102

hipernatural, 44, 102

hiper-racional, 44, 102

hiper-real, 35, 44

inteiro, xvii

irracional, xvii

natural, xvii, 2

racional, xvii, 2

real, xvii

$\mathrm{O}$

Objeto

em $X_{\omega}, 106$

relacionado a $* \mathbb{R}, 36$

relacionado a $\mathbb{R}, 36$

Objetos

definição de, xv

Ordinal, 178

finito, 179

infinito, 179

inicial, 182

limite, 179

sucessor, 179
Palavra, 152

Par

número hiperinteiro, 45

Par ordenado de Kuratowski, xvii

Parte standard

função, 33

Positivo

elemento, 2

Posto

em $\mathrm{V}, 184$

em $X_{\omega}, 110$

Potência reduzida, 84

Primeiro Teorema do Isomorfismo, 18

Princípio

da Boa Ordenação, 178

da Casa dos Pombos, 183

da Definição

Interna (PDI), 48, 115

Standard (PDS), 40, 119

da Indução, 180

Interna, 50

Transfinita, 178, 179

da Transferência (PT), 39, 111

da Transferência para $\prod_{\mathcal{U}} \mathbb{R}, 104$

do Overflow, 131

do Underflow, 131

Geral das *-Transformações (PGT), 119

Produto

cartesiano, xix, 177

finito, xviii

direto, 85

reduzido, 84

Propriedade

Arquimediana, 14

das Interseções Finitas (PIF), 79

do Supremo, 21 
Q

Quociente

conjunto ordenado, 16

de um conjunto, xix

domínio ordenado, 17, 18

$\mathbf{R}$

Recursão Transfinita, 180

Redução de uma estrutura, 161

Relação

de equipotência, 181

de pertinência, xv

de pertinência em uma classe, xviii

de proximidade finita, 19

de proximidade infinita, 19

de satisfatibilidade, 164

domínio de uma, xviii

imagem de uma, xviii

S

Saturação, 148

Sentença, 159

Sequência, xvii

Série de Laurent, 7

Símbolo

da conjunção, 155

da disjunção, 155

da igualdade, 155

da negação, 155

da quantificação existencial, 155

da quantificação universal, 155

da vírgula, 155

de constante, 153

de variável, 155

do parêntese, 155

específico, 155

funcional, 153

lógico, 155

relacional, 153

Soma de dois ordinais, 180
Standard

objeto, 39, 114

Subassinatura, 154

Subcorpo ordenado, 8

Subdomínio ordenado, 3

Subestrutura, 167

elementar, 167

Subfórmula, 157

Subpalavra, 152

Substituição simultânea, 152

Subtermo, 156

Sucessor

de um cardinal, 183

de um ordinal, 179

Superestrutura, 111

Supremo, 21

$\mathrm{T}$

Teorema, 166

da Função Inversa, 64

de Bernstein, 181

de Bolzano-Weierstrass, 62

de Cantor, 183

de Cauchy, 62

de Łoś, 93

de Hartog, 182

de Zermelo, 182

do Ponto Crítico, 63

do Valor Intermediário, 127

Fundamental do Cálculo, 72

Fundamental dos Ultraprodutos, 93

Teoria, 166

Teoria dos Conjuntos

de Neumann-Bernays-Gödel (NBG), 174

de Zermelo-Fraenkel (ZF), 172

de Zermelo-Fraenkel-Choice (ZFC), 173

Termo, 155

fechado, 156

livre para uma variável, 159

Transformação 
de um subconjunto de $\mathbb{R}, 101$

de uma condição fechada, 39

de uma relação em $\mathbb{R}, 102$

U

Ultrafiltro, 89

Ultralimite, 89

generalizado, 89

Ultrapotência, 89

Ultraproduto, 89

União, xvi

enumerável, xviii

\section{Universo}

de uma estrutura, 160

de von Neumann, xvii, 177

V

Valor absoluto, 7

Variável

ligada, 159

ligada em uma posição, 159

livre, 158

livre em uma posição, 158 
